\title{
New Directions in Space Art
}

By

Julian Priest

\author{
A thesis \\ submitted to Victoria University of Wellington \\ in fulfilment of the requirements for the degree of \\ Master of Design
}

Victoria University of Wellington

(January 2021) 


\section{Contents}

1 List of Abbreviations $\quad 7$

2 Glossary 9

$\begin{array}{llr}3 \text { Title } & 12\end{array}$

4 Research Question $\quad 12$

5 Introduction $\quad 12$

6 Methodology 14

7 Field Review 18

7.1 Cultural History of Space $\ldots \ldots \ldots \ldots \ldots \ldots \ldots$

7.1 .1 Overview ....................... 18

7.1 .2 Current Satellites . . . . . . . . . . . . . . . . . . . . . . . 19

7.1 .3 Peaceful Use of Space $\ldots \ldots \ldots \ldots$

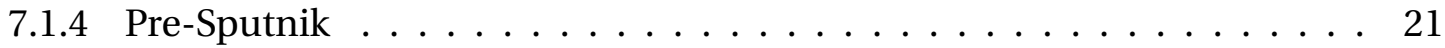

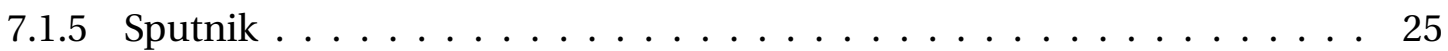

7.1 .6 Post Sputnik . . . . . . . . . . . . . . . . . . 27

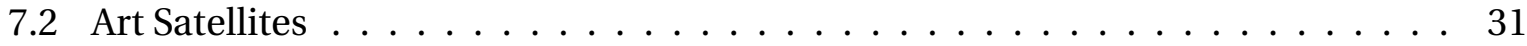

7.2 .1 Overview ....................... 31

7.2.2 Unlaunched Art Satellites (as of 2020) . . . . . . . . . . . . . . . . 31

7.2 .3 Arthur Woods . . . . . . . . . . . . . . . . . . . . 32

7.2.4 G.A.S programme . . . . . . . . . . . . . . . . . . 32

7.2 .5 Eiffel Tower Competition $\ldots \ldots \ldots \ldots$

7.2 .6 Space Art Regulation . . . . . . . . . . . . . . . . . . . 32

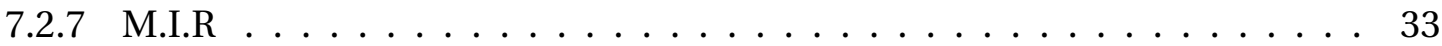

7.2 .8 Ulises . . . . . . . . . . . . . . . . . . . . . . 34

7.2.9 Launched Art Satellites (as of 2020) . . . . . . . . . . . . . . . . . 35

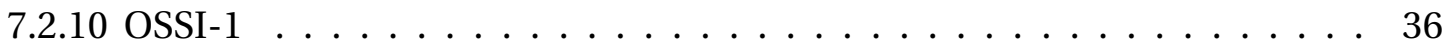

7.2.11 Artsatl: INVADER( $\left(^{*}\right) \ldots \ldots \ldots \ldots \ldots \ldots \ldots$

7.2 .12 Humanity $\operatorname{Star} \ldots \ldots \ldots \ldots$. . . . . . . . . . . . . . . 38

7.2 .13 Red Tesla and Starlink $\ldots \ldots \ldots$. . . . . . . . . . . . 38 
$7.2 .15 \mathrm{ENOCH} \ldots \ldots \ldots \ldots \ldots \ldots \ldots \ldots \ldots$

8 Case Study $\quad 41$

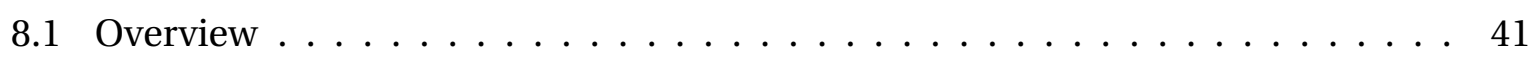

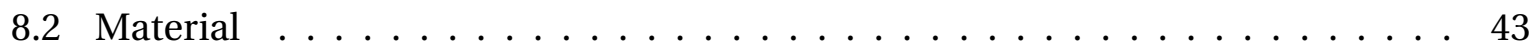

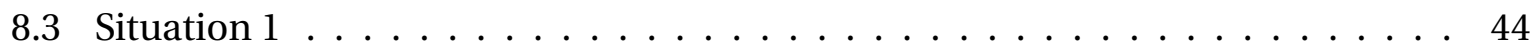

8.3.1 Personal Spacecraft . . . . . . . . . . . . . . . . 45

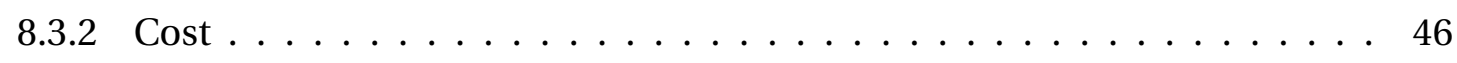

8.3 .3 Form Factor $\ldots \ldots \ldots \ldots \ldots \ldots$

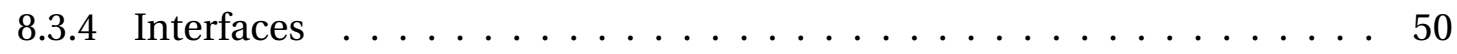

8.3 .5 Community .......................... 53

8.3.6 Development Process . . . . . . . . . . . . . . . . . . 55

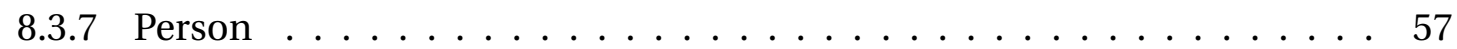

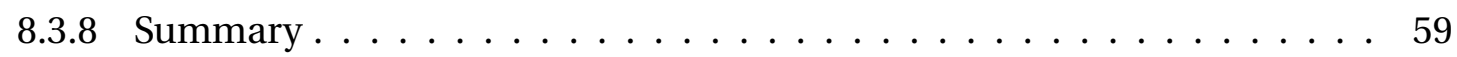

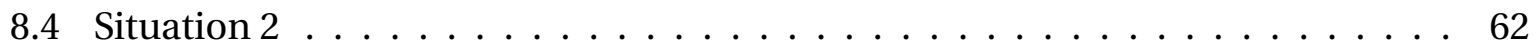

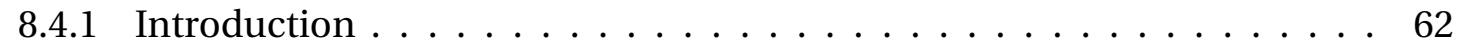

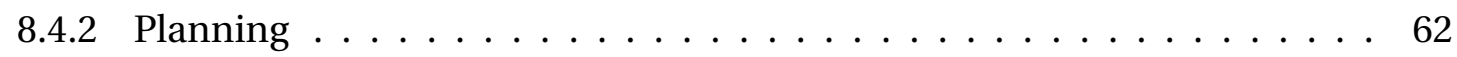

8.4 .3 Dependency . . . . . . . . . . . . . . . . . . . 64

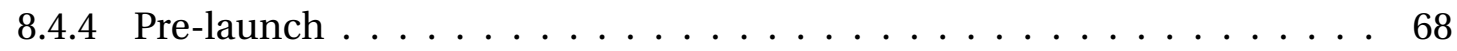

8.4 .5 Meet to Delete . . . . . . . . . . . . . . . . . . . . . 68

8.4 .6 Launch . . . . . . . . . . . . . . . . . . . . 69

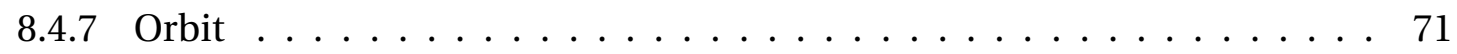

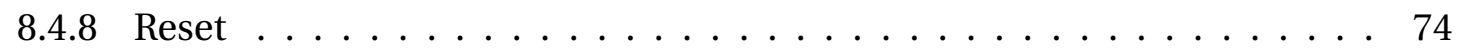

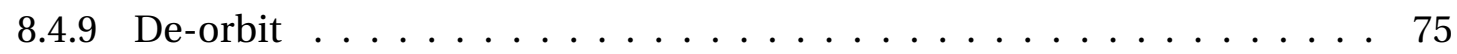

8.4.10 Contingency, Agency and Time . . . . . . . . . . . . . . 79

8.4.11 Discontinuity and Entanglement . . . . . . . . . . . . . 83

8.4 .12 Summary . . . . . . . . . . . . . . . 85

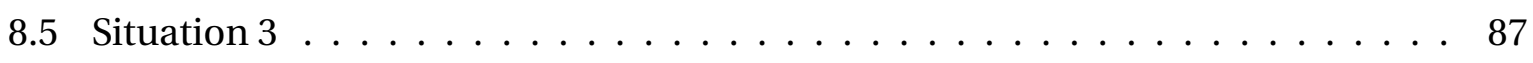

8.5 .1 Radio Spectrum . . . . . . . . . . . . . . . 87

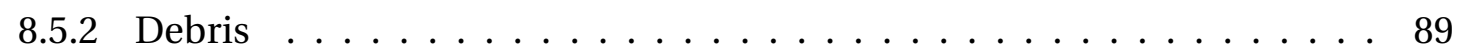

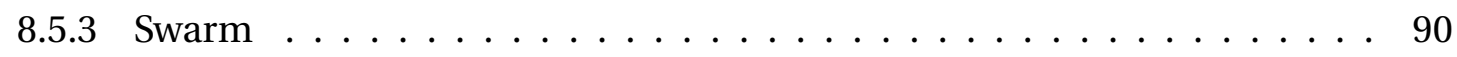

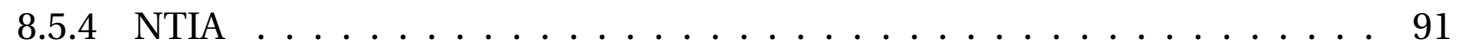




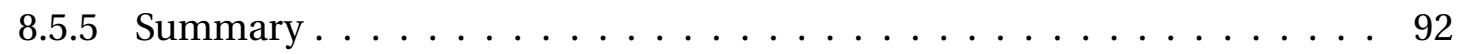

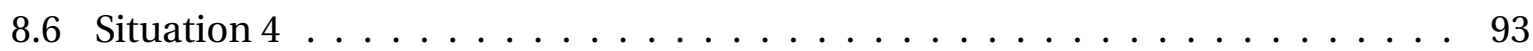

8.6 .1 Visibility ........................ 93

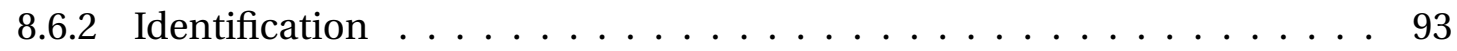

8.6.3 Ephemera . . . . . . . . . . . . . . . . . . . 94

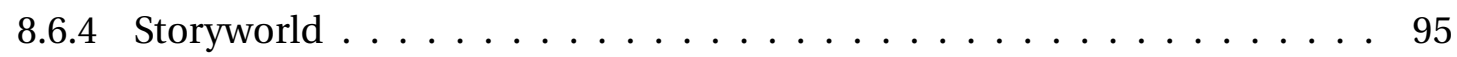

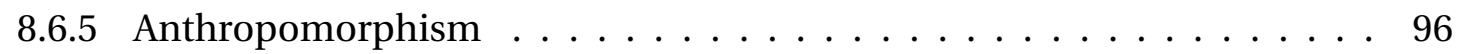

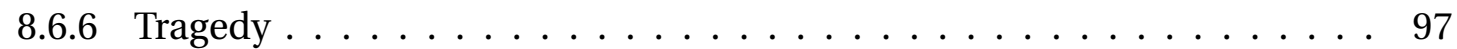

8.6 .7 Line . . . . . . . . . . . . . . . . . . . . 99

8.6 .8 Summary . . . . . . . . . . . . . . . . . . . 104

9 Discussion 105

$\begin{array}{ll}10 \text { Conclusion } & 109\end{array}$

11 References $\quad 111$

12 Appendix A $\quad 124$

12.1 Public Presentations . . . . . . . . . . . . . . . . . . . . . . . 124

$\begin{array}{ll}13 \text { Appendix B } & 157\end{array}$

13.1 Speculative Physics Experiment . . . . . . . . . . . . . . . 157

\section{List of Figures}

1 Functional Satellites by Use. . . . . . . . . . . . . . . . . . . . . 19

2 Whole Disk Images, Brand, DODGE, ATS-3, Apollo $17 \ldots \ldots$. . . . . . . . . . 28

$3 \quad$ OSSI 1 Launch . . . . . . . . . . . . . . . . . . . . . . . . . . . 37

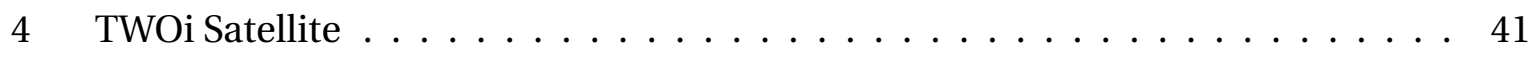

$5 \quad$ Kicksat Kickstarter Page . . . . . . . . . . . . . . . . . . . . . 44

6 Nanosat Forecast . . . . . . . . . . . . . . . . . . . . . . 49

$7 \quad$ Cubesat Acceptance Form . . . . . . . . . . . . . . . . 51

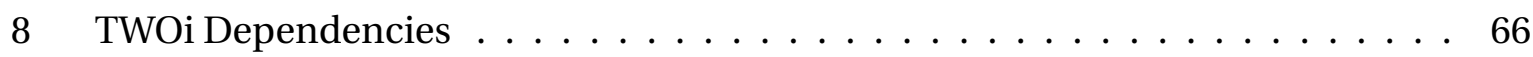

$9 \quad$ CRS-3 Launch $2014 \ldots \ldots \ldots$. . . . . . . . . . . . . . . . . . 70

10 2nd Stage Separation . . . . . . . . . . . . . . . . 73 
11 Kicksat Deployer Voltage . . . . . . . . . . . . . . . . . . . . . . . . . . . 74

12 KickSat-gs Google Group Posts . . . . . . . . . . . . . . . . 76

13 KickSat Decay Prediction Curve May 6, $2014 \ldots \ldots$. . . . . . . . . 77

14 TWOi Re-entry Poster $2015 \ldots \ldots \ldots \ldots \ldots$. . . . . . . . . . . . 78

15 Gell's Protention and Retention . . . . . . . . . . . . . . . . . . . . 80

16 Orbital Debris Jan $12019 \ldots \ldots$. . . . . . . . . . . . . . . . . . 89

17 CGI depiction of KickSat . . . . . . . . . . . . . . . . . . . . 95

18 TWOi poster $2014 \ldots \ldots \ldots$. . . . . . . . . . . . . . . . 100

19 Paul Klee Horizon image . . . . . . . . . . . . . . . . . . . . . 101

20 Paul Klee Meteor trajectory . . . . . . . . . . . . . . . . . . . 102

21 Screenshot of ISAW4 Online Event, $2013 \ldots \ldots$. . . . . . . . . . . 125

22 Ada Symposium 2013 Remote Video Presentation Still . . . . . . . . . . . . . 126

23 Ward Observatory . . . . . . . . . . . . . . . . . . 128

24 Meet to Delete Test Pilot Bonfire. . . . . . . . . . . . . . . . . . . . . . . . . 128

25 Launch Screening Invitation $\ldots \ldots \ldots \ldots \ldots \ldots$

26 Successful Launch Screening . . . . . . . . . . . . . . . . . 131

27 Meet to Delete, Up Galerie, Meta Data . . . . . . . . . . . . . . . . . . . . . 132

28 Meet to Delete, Up Galerie, Deletions ～. . . . . . . . . . . . . . . . . . . . 132

29 Receive and Recycle, Hungary . . . . . . . . . . . . . . . . 133

30 Meet to Delete Whanganui . . . . . . . . . . . . . . . . . . 134

31 Meet to Delete Whanganui bonfire . . . . . . . . . . . . . . . . . . . 134

32 Meet to Delete Ptarmigan . . . . . . . . . . . . . . . . . . . . 135

33 Meet to Delete Pixelache . . . . . . . . . . . . . . . . . . 136

34 Meet to Delete Arizona . . . . . . . . . . . . . . . . . . . . . . 137

35 Meet to Delete Bogota Meta Data . . . . . . . . . . . . . . . . . . . . . . 138

36 Meet to Delete Bogota . . . . . . . . . . . . . . . . . . . . 138

37 Meet to Delete Bogota $\ldots \ldots \ldots \ldots$. . . . . . . . . . . . . . 139

38 TWOi Re-entry Poster f . . . . . . . . . . . . . . . . . . . . . 140

39 Ada Symposium $2014 \ldots \ldots$. . . . . . . . . . . . . . . . 141

40 The Weight of Information Ground Station Layout ～. . . . . . . . . . . . 142

41 The Weight of Information Ground Station Radio . . . . . . . . . . . . . . . 143

42 Thomas King Observatory . . . . . . . . . . . . . . . . . . . . . 144 
43 Space Place Carter Observatory . . . . . . . . . . . . . . . . . . . . . . . . . . 144

44 NG-10 Launch . . . . . . . . . . . . . . . . . . . . . . . . . . . . . . . 145

45 NG-10 Cygnus Flight $\ldots \ldots \ldots \ldots \ldots \ldots \ldots$

46 NG-10 ISS Docking . . . . . . . . . . . . . . . . . . . . . 146

47 TWOi 2.0 Video . . . . . . . . . . . . . . . . . . . . . . . . . . . 147

48 TWOi 2.0 Mission Control Room . . . . . . . . . . . . . . . . . . . . . . . . 148

49 TWOi 2.0 Satellite Balance . . . . . . . . . . . . . . . . . . . . . . . . . . . 148

50 TWOi 2.0 Telemetry . . . . . . . . . . . . . . . . . . . . . . . . . . . . . 149

51 TWOi 2.0 Antenna Rotator . . . . . . . . . . . . . . . . . . . . . . . . . . . . 149

52 Meet 2 Delete Paper Shredding . . . . . . . . . . . . . . . . . . 150

53 Meet 2 Delete Confetti Cannon . . . . . . . . . . . . . . . . . . . . . . 151

54 Meet 2 Delete Paper Shred Drift . . . . . . . . . . . . . . . . . 151

55 Tape Measure Dance Performances . . . . . . . . . . . . . . . . . . . . 152

56 Dwingeloo Radio Telescope . . . . . . . . . . . . . . . . . . 153

57 Kicksat Sprite Transmission including TWOi 2.0 . . . . . . . . . . . . . . . . . 154

58 TWOi Flies Free Poster . . . . . . . . . . . . . . . . . . . . . . 155

59 TWOi 2.0 Re-entry Prediction . . . . . . . . . . . . . . . . . . . 155

60 Kicksat Sprite Satellite . . . . . . . . . . . . . . . . . . . . . . 157

61 Kicksat Sprite Transmission including TWOi $2.0 \ldots \ldots$. . . . . . . . . . . 158

62 TWOi PRN 26 detection trace . . . . . . . . . . . . . . . . . . . . 159

\section{List of Tables}

1 Unlaunched Art Satellites $\ldots \ldots \ldots \ldots \ldots \ldots$

2 Launched Art Satellites . . . . . . . . . . . . . . . . . . . . . 35

3 Satellite Launch Costs $\ldots \ldots \ldots \ldots \ldots \ldots$. . . . . . . . . . . 47

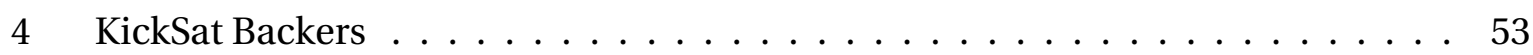

5 KickSat Launch Evolution . . . . . . . . . . . . . . . . . . . 64

6 Space Weather April, $2014 \ldots \ldots \ldots \ldots \ldots \ldots \ldots \ldots$

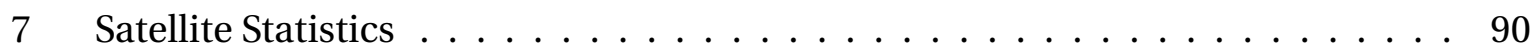




\section{List of Abbreviations}

ATS-3 Applications Satellite - 3

BIS British Interplanetary Society

CERN European Organization for Nuclear Research

COTS Commercial Orbital Transportation Services

CRS, CRS-1, CRS-2, CRS-3 Commercial Resupply Service

DARPA The Defense Advanced Research Projects Agency

DODGE Department of Defense Gravity Experiment

EIRP Effective Isotropic Radiated Power

ELaNa Educational Launch of Nanosatellites

EOF End of File

EOL End of Life

ESA European Space Agency

FCC Federal Communications Commission

GPS Global Positioning System

IAAA International Association of Astronomical Artists

IAU International Astronomical Union

ICBM Intercontinental Balistic Missile

IDE Integrated Development Environment

IGY International Geophysical Year 1957/58

ISRO Indian Space Research Organisation's

ISS The International Space Station

JAXA Japan Aerospace Exploration Agency 
ka BP thousand years before present time

LACMA The Los Anegles County Museum of Art

LEO Low Earth Orbit

NASA National Aeronautics and Space Agency

NORAD North American Aerospace Defence Command

NTIA National Telecommunications and Information Administration

ODA Orbital Debris Assessment

OSSI The Open Source Satellite Initiative

PAGEOS Passive Geodetic Earth Orbitting Satellite

PAROS Prevention of Arms Race in Space

PPOS Poly Picosat Orbital Deployer

PPWT Preventions of the Placement of Weapons in Outer Space

PS-1 Prosteishiy Sputnik 1, (Simplest Satellite 1)

PSLV Polar Satellite Launch Vehcile

SAA South Atlantic Anomaly

SSDS Space Systems Design Studio at Cornell University

TEU Twenty Foot Equivalent

TKO Thomas King Observatory

TLE Two Line Element

TWOi The Weight of Information

UCUSA Union of Concerned Scientists

UNOST United Nations Outer Space Treaty

USAF United States Air Force

USSF United States Space Force 


\section{Glossary}

$1 U, 2 U, 3 U$ Shorthand for mutliples of $10 \times 10 \times 10 \mathrm{~cm}$ unit height in the CubeSat nano satellite form factor definition. A $2 \mathrm{U}$ satellite is $20 \mathrm{x} 10 \mathrm{x} 10 \mathrm{~cm}$.

Apollo/Soyuz The 1975 rendezvous in orbit between an Apollo and a Soyuz spacecraft, it was the first international space mission and laid the foundations for the ISS.

Arduino A popular open source micro-controller board family integrated software development environment.

Boot The process of starting a computer.

$\mathrm{C}, \mathrm{C}++$ Programming languages.

Celestrak A web service that publishes and updates satellite positions and trajectories.

Chipsat A very small satellite based on an exposed circuit board.

Cubesat A popular standard miniature satellite specification and form factor in multiples of 10x10x10cm. The design was propopsed in 1999 with 1100 deployments as of 2020.

DARPA DARPA was a research agency that was responsible for the early development of computer networks that led to the Internet.

Echo A US passive reflective inflateable satellite communications program between 1960 and 1964 that produced a 30m and a $41 \mathrm{~m}$ satellite.

Electron Electron is the primary orbital launch vehicle of RocketLab.

Energia An integrated software development environment for MSP430 based on the Arduino IDE

Falcon The primary rocket of Space-X used in various configurations based on the same modular rocket technology.

femtosat a very small $<5 \mathrm{~cm}$ satellite

Github A public software repository, and collaboration platform often used for open source software, based on the git version control system. 
Gold Code A mathematically proven fault tolerant binary sequence that enables decoding of partial signals thereby improving gain through software processing.

Google Group A free email list service operated by Google.

Gpredict Open source satellite prediction software.

HackSatOne A UK based Kicksat development team.

Karman Line A definition of the edge of outer space approximately $100 \mathrm{~km}$ above Earth's surface proposed by Theodore von Kármán. It is defined as the height at which a vehicle's speed to acheive sufficient aerodynamic lift is equal to the orbital velocity.

Kessler Cascade A potential disaster scenario in which orbital debris impacts and destroys a satellite creating further orbital debris which in turn impacts other satellites.

KickSat Zachery Manchester's femto satellite project.

KickStarter An online crowdfunding platform founded in 2009.

Maxwell A cubesat dispenser system by Rocketlab.

Meet 2 Delete Public event series accompanying 2018/2019 TWOi orbit.

Meet to Delete Public event series accompanying 2014 TWOi orbit.

MSP430 A low power microcontroller by Texas Instruments with built in transmitter.

Nanoracks A US space services corporation that operates the Nanoracks Cubesat Deployer (NRCSD) system on the ISS.

New Space A characterisation of contemporary space exploration that emphasises the private, entrepreneurial and low cost style of space exploration in contrast to Old Space era led by nation states.

Open Source Software Software in which source code is released under copyright licenses that encourage modification and sharing.

PAGEOS A 30m reflective mylar balloon satellite launched in 1966.

PhoneSat A satellite flight computer project based on modified smart phones. 
Picaxe A microcontroller based on the PIC standard popular with hobbyists.

R7 R-7 Semyorka is family or rockets developed by the USSR as ICBMs that became the basis for the most launched heavy lift orbital delivery rockets including Soyuz.

Radio Ham A radio amateur.

Rocketlab NZ/US orbital launch company with launch sites in Mahia, New Zealand, and Wallops, Virginia, USA.

Saturn V NASA's heavy-lift launch vehicle used in the Apollo space program that took astronauts to the moon.

Scrub The cancellation of a rocket launch.

South Atlantic Anolmaly An area of disturbance in the Earth's magnetic field centered on the South Atlantic that reduces the altitude of the Van Allen radiation belt thus allowing increased cosmic ray flux at Earth's surface.

Space-X A privtae US space transportation company founded in 2002.

Sprite An individual KickSat chip satellite.

Starlink LEO satellite constellation of potentially thousands of satellites being deployed by Space-X since 2019 for internet provision.

SWARM A company providing satellite based network connectivity for Internet of things devices using small form factor satellites.

Tesla US based electric car company whose CEO is Elon Musk, also CEO of Space-X.

$\mathrm{T}$ Rocket launch take off time which launch sequence events are measured relative to i.e $\mathrm{T}$ minus 10 .

Tubesat A pico satellite form factor promoted by Interorbital Systems.

Unix A widely used family of multi-tasking, multi-user computer operating systems developed since the 1970s

ZX81 Sinclair ZX81 low cost home computer produced in 1981. 


\section{Title}

New Directions in Space Art

\section{Research Question}

"How are Space Art projects enabled by new developments in the space industry?"

\section{Introduction}

The space industry has entered a period of rapid change that is characterised by increased activity of private corporations. Commercial launches are becoming common place as opposed to a previous era where launch was controlled by state space agencies. Other new developments include new and cheaper launch platforms, new space cargo form factors, new legal jurisdictions and the development of new off the shelf space craft technologies. These developments are portrayed as lowering barriers to entry into the space industry and opening the field up to a wider range of participants.

These changes in access relations in turn are said to be creating a wide range of new opportunities for smaller groups and individuals to participate in space exploration. These new opportunities may be used by artists and designers to engage in cultural projects that use outer space as either a primary subject matter or as a site.

In this thesis I examine to what extent these new space access relations have made an impact on contemporary art practice with a particular focus on the field of satellite artworks. Further I examine which specific features of these new access relations are significant, and which are secondary.

To provide overall context I provide a historical overview of cultural use of space which argues that cultural and artistic use of space has been important in the development of space exploration. I continue this contextual section to include a brief review of all art satellites, planned, failed and successfully realised in the era of space exploration. The field review section is aimed to provide a field delineation for art satellites that is inclusive of other disciplines within the broader culturisation of space.

With this framework in place, the core of the paper consists of a case study of a recent space art work by myself, The Weight of Information. This project took place between 2009 and 
2019 and involved two different satellite launches that were provided through participation in the KickSat programme. The work consisted of a large number of artistic and cultural events, one failed deployment in 2014 and a successful one in 2018/19.

The methodology uses both auto-ethnographic and design-ethnographic techniques as well as self-reflection, documentation and media artefacts of the project to create four detailed narrative situations. This situational approach is exploratory, and relevant themes are then drawn out from the narrative to produce a theoretical framework for each situation.

The first section covers the development process and examines the tension between personal and infrastructural, the second describes the launch evolution in terms of contingency, dependency and entanglement, the third looks at the wider regulatory environment and the last examines artistic approaches to orbital inaccessibility.

The work is highly interdisciplinary and of relevance to artistic, cultural or design projects in orbit using the new access relations, as well as to the New Zealand national context since the recent formation of Rocketlab. 


\section{Methodology}

This thesis presents practice-led art-science research that takes the form of an extended case study and locating field review. The research uses auto-ethnographic and design-ethnographic approaches to describe novel and improvised situations, settings and processes in satellite art. The case-study is based on primary material drawn from a single satellite art-research project by the author, and this is located within contemporary space art and the broader field of the culturisation of space.

The core material for this research project is the output from the Weight of Information [TWOi] satellite art project. TWOi took place between 2009 and 2019 and explored the relationship between information and gravitation through speculative experimental art-science research. The TWOi project can be primarily considered as an artwork in the field of space art. It was realised as a large number of events and public outputs, as well as a significant number of documents, objects, installations, media artefacts, and a speculative scientific experiment. This body of work is considered here as a piece of artist-science research and its outputs form the main research material of this thesis.

What is presented however does not directly concern the artistic research question that was explored during the TWOi project, namely 'What is The Weight of Information?' or look at the specific intersections of physics, art and network culture that are explored in that project. Instead the research looks at some of the processes, opportunities and challenges that were encountered during the making of the project, and this focus on process expands the relevance of the thesis to the wider field of cultural projects and design.

Further the thesis locates the work by delineating a field of satellite art within the wider cultural history of space exploration. This develops context and is achieved through an extended field review that includes literature, a range of artworks and other cultural and scientific projects.

TWOi was a novel satellite art project that was made possible by an open-source and crowd-funded community space research project that was launched on a private commercial rocket. Each of these aspects are in themselves new, and together form an operating environment that has unique features for designers, artists and cultural projects in the developing field of the culturisation of space. There have been very few art satellites to date ( 4 as of $2019^{1}$ ) and so there is no large body of pre-existing work describing the processes

\footnotetext{
${ }^{1}$ The exact number is dependent on how you categorise art satellites. Here I count satellites produced pri-
} 
covered in this thesis.

In considering an appropriate research methodology, the main features of the material produced by the TWOi artwork are considered. The artwork was completed before the start of the thesis and so the research is retrospective in nature. The work concerns artisticresearch and therefore should take account of practice. Next the work was undertaken by the author so the methodology should take into account the role of the author in the project and the subjective position within the project. To balance this subjective positioning, a form of critical distancing should be included. The project took place in a network of relationships with audience, institutions and another key projects and so a technique that foregrounds these relationships is necessary. Lastly TWOi was a highly interdisciplinary artscience project crossing over elements of scientific experiment and technique with cultural and artistic forms, and so a methodology that can span a broad range of fields is needed.

The thesis is concerned with practice, but is not considered practice-based rather being closer to practice-led research. Practice-based research is concerned with the output of an artistic or design research programme, whereas this thesis concerns the process by which an artistic research project proceeded and presents what can be learned from this process. According to Earnshaw (2016) "Practice-led Research is concerned with the nature of practice and leads to new knowledge that has operational significance for that practice"(p.24). However the thesis does look back at an existing project, and so while it looks at practice, it is not produced directly by practice and instead takes the form of an extended case study.

The source material is both retrospective and auto-biographical and the research aims to draw out relations that existed in the development process of the TWOi project. The research is in general exploratory and qualitative and quantitative research techniques were therefore discounted at an early stage.

As TWOi was a project by the author, the research material is necessarily subjective and personal. Auto-ethnography was therefore taken as a starting point in order to make this subjectivity a positive feature rather than a hindrance. Ellis, Adams, and Bochner (2010) highlight the retrospective nature of auto-ethnography:

When researchers do auto-ethnography, they retrospectively and selectively write

marily as dedicated artworks, that flew successfully and autonomously in orbit as designed. A number of satellites have malfunctioned and a number have had multiple purposes, including as propaganda, advertising. 
about epiphanies that stem from, or are made possible by, being part of a culture and/or by possessing a particular cultural identity. (p.4)

However auto-ethnography typically focuses on changes that are brought about in the author as a result of their experiences, and this is not the sole focus here. The research looks at an art form, space art and how conditions of access to space enable or disable that art form. These conditions are created by social, cultural, network and organisational relations and these are a core focus of design ethnography.

The Weight of Information was highly dependent on the KickSat project which provided the opportunity for the project to be realised. KickSat provided the satellite, software, hardware, launch, base station design and technical community that TWOi was built on. KickSat itself had a large number of organisational dependencies inherent in working with space based projects. The interactions with this project and the larger network of space based projects is therefore key in explaining the process by which TWOi came into being. From a methodological point of view, while the research is sited in TWOi, much of the analysis is of the process and interaction with the KickSat project and its externalities. In this thesis the relationship between the TWOi project and KickSat is akin to a straight ethnographic relationship, with the TWOi tent set up at the edge of the KickSat village.

The ethnographic nature of the research poses a problem of subjectivity and authorship. As this study is necessarily concerned with a project that was also authored by the primary researcher, there is a potential for a lack of critical distance. To address this from a methodological point of view, some techniques of self-reflective practice are used. Schon (1983) describes the wide variety of objects of reflection:

When a practitioner reflects in and on his practice, the possible objects of his reflection are as varied as the kinds of phenomena before him and the systems of knowing-in-practice that he brings to them. He may reflect on the tacit norms and appreciations that underlie a judgment, or on the strategies and theories implicit in a pattern of behaviour. He may reflect on the feeling for a situation that has led him to adopt a particular course of action, on the way in which he has framed the problem he is trying to solve, or on the role he has constructed for himself within a larger institutional context. (p.62)

Within the field of design ethnography this subjective and engaged stance is often framed as participant observation. Within Design Anthropology Gatt and Ingold (2013) contextu- 
alise this method and develop a concept of anthropology-by-means of design that rests on the idea of correspondence. "To correspond with the world is not to describe it, or to represent it, but to answer to it."(p.135) Further they describe the creativity of design as proceeding by improvisation in response to situations in the world rather than innovation. This dynamic accurately frames the emergent development of the TWOi project from its relations and is therefore a useful methodological lens to view the project through.

The TWOi artwork was not originally conceived as a satellite work, and it was heavily shaped by the operating environment, of both the parent project and the network of organisations that surrounded it. At every step of the project challenges, opportunities and influences were encountered which changed the path and character of the work. In this sense TWOi can be thought of as a highly reflexive and iterative project which came to fruition through a decade of negotiation and development. Therefore rather than considering TWOi as an individually authored creative project and analysing it in terms of creative intent, position within an artistic tradition or as situated in one or other theoretical frameworks, TWOi is considered to be emergent from its process.

The TWOi project was clearly conceived as an interdisciplinary art-science project crossing over elements of scientific experiment and rationale with cultural and artistic forms. In TWOi elements of both scientific experiment, performance and participatory art are present and ethnography and design provide frameworks for examining these often disparate forms.

The main body of the research therefore comprises a series of specific situations that TWOi was emergent from. Each situation is rooted in a particular moment in the project which is explored through a detailed description in the case study. These descriptions enumerate the interactions of entities that have agency in the situation, such as people, technologies, organisations or objects. Mitchell (2006) describes a social situation as "a collocation of events which the analyst is able to construe as connected with one another and which take place in a relatively restricted time span." (p.28)

These situations take place in a wider cultural setting, that comprises both the institutions, individuals and groups involved but also the various cultures and regulatory environments surrounding the project. Analysis of each situation is done with respect to a particular framework that is suitable for that situation and engages directly with the differing fields on their own terms, and through their own techniques and literature. 


\section{Field Review}

As space artist Hujon Song's OSSI-1 (2013) art satellite blasted off from Baikonur Cosmodrome, he wore his trademark sash, inscribed with the words Science is Fantasy (Song, 2014b). The slogan highlights the imaginative work that comes in advance of theoretisation and experimentation in science. Rocket theoretician Konstantin Tsiolokvsky embodied Song's slogan in his career. After starting in science fiction he turned to the mathematics of rocketry, producing key equations that are used to this day. Before Sputnik there was no access to space, and all space exploration was fantasy, imagined in culture thousands of years before it was realised. Once orbit had been achieved a different geopolitical dynamic took over and cultural projects took a back seat. It is only very recently as conditions of access to space have begun to change, that artists have been able to use dedicated satellites as material for artworks and launch them into orbit.

This field review section argues for the importance of cultural use of space, and space art, and then delineates a contemporary field of satellite art. Consequently the section is split into two, first a brief cultural history of space to provide context, and second a review of previous and current satellite artworks.

\subsection{Cultural History of Space}

\subsubsection{Overview}

The current human use of outer space is predominantly peaceful and the majority of satellites in orbit around Earth are for commercial or scientific use. Cultural use of space is very limited making up only a microscopic fraction of overall space use and it has been at times actively excluded, critiqued and viewed with suspicion by both other space users and the general public. And yet, cultural and artistic practice have had an extremely important role in the historical development of space exploration. Here I therefore wish to redress this imbalance by tracing a deliberately cultural history of space exploration that emphasises these cultural and artistic interventions at key moments, in order to argue for an expanded future role for cultural projects in space. This is by no means a definitive history, but rather a partial and selective one that is presented here to emphasise the cultural aspects in order to position the later discussion on satellite art, and the case study within an appropriate historical context. 
It is split into three eras, pre-Sputnik, Sputnik, and post-Sputnik. At each stage the conditions of access to space are considered and their effect on particular artworks explored. In the pre-Sputnik era works, by Lucian and Gaillelo establish an early imaginary for space flight. Artistic contributions by the Russian Cosmists, Fedorov, Tsiolokovsky and Malevich are then linked to the eventual launch of Sputnik. I discuss Sputnik itself considered from a predominantly cultural point of view, and in the post Sputnik era focus on scientific images co-opted as artworks, the clandestine moon sculptures of the Apollo era, and the emergence of artworks created deliberately for the space environment. This sets the scene for the second contextual section which is a comprehensive survey of satellite artworks.

\subsubsection{Current Satellites}

In 1967 the UN Outer Space treaty, which mandated the peaceful use of Space, was signed. At that time all space exploration took place in the public sectors of different nations states, in scientific, civil or military capacities. In the 1970s, while launches remained state operated, a private commercial sector was developed, especially in telecommunications and broadcast, and as of 2020 over half of all functioning satellites were commercial.

Currently (as of 2020) there are 2667 satellites of which 2181 are commercial, governmental or scientific and 486 are for non-belligerent military use, (Celestrak.net, 2020; Ucsusa.org, 2020). By contrast cultural use appears to be under-represented and there is only a single active art satellite in orbit, Tavares Strachan's Enoch (Strachan, 2017).
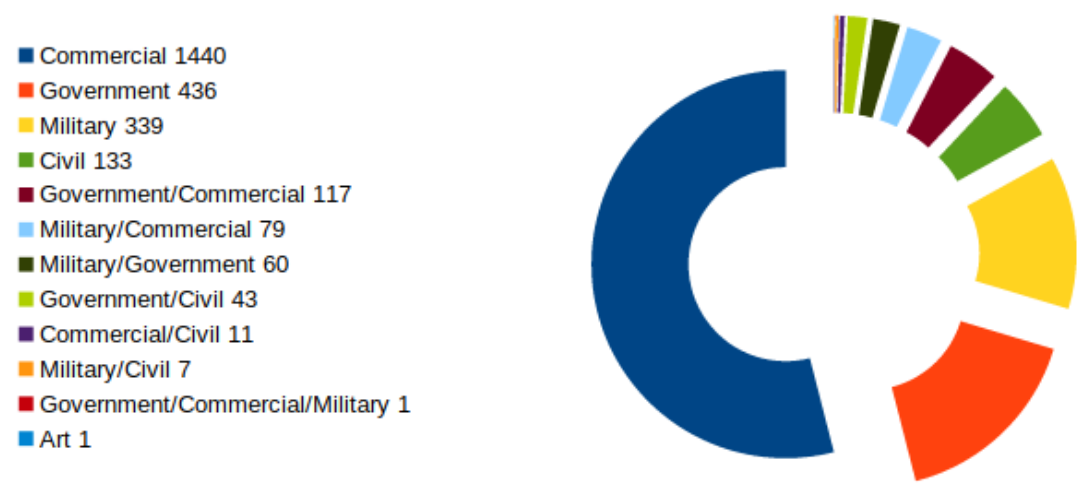

Figure 1: Functional Satellites by Use.

Note. Data compiled from online databases (Celestrak.net, 2020; Ucsusa.org, 2020). By Julian Priest. 


\subsubsection{Peaceful Use of Space}

Common histories of space exploration emphasise the primacy of military use and trace the development of the rocket to its origins in warfare. This type of history highlights for instance the appearance of the fist documented rockets in 10th century China as propulsion systems for arrows, and the rockets used by India in the Anglo-Mysore wars, and by Britain during the fire bombing of Copenhagen in 1807. The space race is often connected with the military technology of the Inter Continental Ballistic Missile (ICBM), and episodes such as the transition of engineers such as Von Braun from V-2 rocket engineer to Saturn V designer. In this style of history the orbital access rocket is seen primarily as an attempt to occupy the ultimate military high point (Burrows, 2010).

While many of these historical perspectives are valid, and the first decade of space exploration did take place in the context of the cold war, the predominant use of outer space has nevertheless been peaceful. During the post-war period there was an attempt to avoid future widespread conflict through multilateral treaties and organisations some of which restricted future research directions in the nuclear fields. In 1953 the foundation of the European Organisation for Nuclear Research (CERN) aimed to create a large scale experiment that would direct the global community of nuclear scientists towards non-military research with peaceful goals such as the search for fundamental physical principles. "The Organization shall have no concern with work for military requirements and the results of its experimental and theoretical work shall be published or otherwise made generally available." (Council, 1953)

This approach successfully re-framed atomic physics away from a solely military agenda, held the possessors of nuclear knowledge in a transparent public structure and gave access to this knowledge to emerging atomic nations within a context of international dialogue.

A similar process was initiated after the US Starfish Prime orbital nuclear test damaged satellites from many nations with an electromagnetic pulse in 1962. The United Nations Outer Space Treaty (UNOST) was drafted and signed in 1967 thus ending a decade of potential conflict that began with Sputnik in 1957. It placed restrictions on the use of outer space, prohibiting the use of nuclear weapons in space, and designating the moon for only peaceful activities.

"States Parties to the Treaty undertake not to place in orbit around the Earth any objects carrying nuclear weapons or any other kinds of weapons of mass destruction, install such weapons on celestial bodies, or station such weapons 
in outer space in any other manner.

The moon and other celestial bodies shall be used by all States Parties to the Treaty exclusively for peaceful purposes." Outer Space Treaty (1966)

Since the treaty there have been significant developments of military systems that use satellite technology such as GPS, military communications, spy satellites and the strategic defence initiative, but no acts of war to date. Very recently there have been a range of antisatellite proof-of-capability ballistic tests by China (2007), US (2008), India (2019) and Russia (2020). Non-proliferation treaties to strengthen UNOST - Prevention of Arms Race in Space (PAROS) and Prevention of the Placement of Weapons in Outer Space (PPWT) - have been in draft stage with the UN since 2008. Some resolutions were adopted in 2014, but the treaties are currently blocked primarily by the US. With the current international retreat from multilateral obligations and the 2019 creation of the United States Space Force (USSF) ${ }^{2}$, it is unclear (as of 2020) how long the peaceful situation in outer space will last (Johnson-Freese \& Burbach, 2019).

Peaceful use of outer space includes governmental, scientific, commercial and increasingly private use, but here I will focus on cultural use in the history of space exploration.

\subsubsection{Pre-Sputnik}

To look up in awe at the stars in the night sky on a clear night and wonder about our place in the universe is a shared experience that unites people from all places and all times. Until very recently in human history, the objects in the night sky were the fixed stars, the wandering stars or planets and the occasional meteor, comet and aurora. In the 21 st century we also see the reflections from the bodies of satellites, such as the dotted lines of the Starlink communication satellite constellation, that show evidence of human activity in space visible to the naked eye. Meanwhile the combination of indoor living, artificial lighting and pollution have reduced the daily experience of the stars for many urban dwellers. Long before the mechanics of orbits were settled by the Copernican revolution, and access to space was finally achieved in 1957, cultural artefacts were produced about astronomical events.

Archaeosastronomical studies show evidence of extremely early depictions of astronomical subjects in cave paintings and artefacts during the Upper Paleolithic era (40-12ka BP), such as the drawings in the Cave of Lascaux and bone engravings from the Cave of La Vache

\footnotetext{
${ }^{2}$ The US Space Force was created as a separate command of the US military 2019, December 20.
} 
(17ka BP). The earliest astronomical artefacts and installations often concerned calculation of seasonal events of importance such as hunting (Ruggles, 2015).

1. Icaromenippus

It is impossible to say when the possibility of human space flight was first considered. It appears first in literature in the 2nd century $\mathrm{AD}$ when the satirist Lucian wrote the dialogue of Icaromenippus or The Sky-Man, in which Menippus flies into space and views the Earth from the moon.

When I approached the Moon, long after parting from the clouds, I was conscious of fatigue, especially in the left or vulture's wing. So I alighted and sat down to rest, having a bird's-eye view of the Earth, like the Homeric Zeus. (Lucian, 2014)

From this godlike vantage point Menippus is able to examine in detail the affairs of humans on the ground as if they were ants and provide a critical commentary on the society of the day. Writing at the time of Ptolemy, Lucian's view of the universe was geocentric and Aristotelian, with Moon, Sun and heavens arranged in distance from the Earth in ascending levels of divinity. Menippus is here a satirical character who transgresses the boundary between human and divine.

2. Galileo

During the Scientific Revolution the view of humankind's place in the Universe shifted to a heliocentric one, first based on Copernican circular orbits and later on Keplerian ellipses. Galileo is the key political figure in this period, and he came into conflict with the Roman Catholic Church, which was heavily invested in Ptolemaic geocentrism. The philosopher of science Feyerabend (1988), argued that at the time the geocentric description gave better scientific predictions of observation than the new heliocentric model, and that Galileo was maintaining an incommensurable counter example on the basis of scientific conviction. In this light the conflict was at least partly political in nature and these political aspects are supported by Galileo's literary and artistic representations. In 'Dialogue On The Two Great World Systems' the geocentric theory of the Pope is voiced by a character called Simplicio who is opposed to heliocentrism (Finocchiaro, 1997). Simplicio translates to simpleton in Italian and some authors pinpoint this caricature as a turning point in the affair. In earlier work Galilei (2016) pro- 
duced 'Drawings of the Moon' from his early telescope observations whose naturalism is highlighted by Winkler and Van Helden (1992).

The drawings depict shadows of mountains and valleys which show the Moon to be a planet like Earth. The depiction of the moon as worldly rather than divine transgressed the Ptolemaic divinity of the celestial realm. Taken together the satirical text and the naturalistic illustrations demonstrate the use of artistic forms in support of a new scientific theory, and for Feyerabend at least these cultural forms and the political shift that accompanies them, takes place in advance of scientific observational confirmation.

\section{Russian Cosmism}

Nikolai Fedorov was a mid 19th century Russian Philosopher whose followers, the Russian Cosmists, were influential in developing the utopian idea of human expansion into space. Art historian Groys (2018) describes how Fedorov's Cosmism had its origins in a particular view of art as preservative rather than progressive. This led to a radical equality with the goal of preservation of all lives past and present, and this in turn necessitated spreading humanity beyond Earth's bounds. Variations of these ideas were developed by different Cosmists and strongly influenced the Russian avant-garde.

A colleague of Fedorov's, the mathematician Konstantin Tsiolkovsky in 1903 first rigorously analysed the mathematics of rocketry ${ }^{3}$. He calculated the minimal viable orbit, as well as developing the rocket equation which describes how to achieve it. A version of the equation is still in use today for calculating the orbital dynamics, fuel loads, and escape velocities of rockets. His seminal work was studied intensively by the later pioneers of rocketry, Von Braun and Korolev.

$$
\Delta_{v}=v_{e} \ln \frac{m_{0}}{m_{f}}
$$

Tsiolkovsky worked for 3 years with Fedorov at the Chertkov Library and initially was

\footnotetext{
${ }^{3} \mathrm{He}$ is preceded by the lesser known William Leitch in first describing the possibility of space flight by rocket in literature.

"The only machine independent of atmosphere, we can conceive of, would be one on the principle of the rocket" Godwin (2016); Leitch (2012)
} 
interested in writing science fiction on cosmist topics, publishing two booklets during this period On the Moon and Daydreams of Earth and Heavens (Suvin, 1971). He returned to write more substantial science fiction titles later in life after the Russian revolution.

Tsiolkovsky acknowledged the strong linkage between literature and science both in his inspiration and his own scientific practice.

I do not remember how it got into my head to make the first calculations related to rocket. It seems to me the first seeds were planted by famous fantaseour, J. Verne. (Hagler, 2013)

First, inevitably, the idea, the fantasy, the fairy tale. Then, scientific calculation. Ultimately, fulfillment crowns the dream. (Crouch, 2000)

In Tsiolkovsky we see a highly interdisciplinary thinker whose career started in literature before making significant contributions to the mathematics of achieving space flight, that are themselves inspired by literature.

Another of the Cosmists, Alexander Chizhevsky - who later worked with Tsiolkovsky - did detailed research on solar activity and wished to correlate the timing of mass human political and social events with the eleven year solar maxima and minima cycle. While his work was speculative there is a correlation between solar activity minima and increased cosmic ray flux as well as upper atmosphere ozone production. Groys (2018) associates Chizhevsky's themes with the futurist mystery opera Victory Over the Sun, which celebrated the extinction of the Sun and a descent into chaos. At the centre of the stage set was Malevich's Black Square painting (Malevich, 1915), described by many as the painting which negated all previous painting, but here Malevich clearly relates it outer space. "'My new painting,' he wrote, 'does not belong exclusively to the earth.... And in fact, in man, in his consciousness, there is a striving towards space, a yearning to 'take off from the earth'." (Kovtun \& Douglas, 1981, p.4)

As I will explore in situation 4, Malevich was to go on to himself propose orbital satellites artworks and to coin the name Sputnik which was later used for the first artificial satellite ${ }^{4}$. "A new Sputnik can be built between Earth and Moon, a Suprematist Sputnik equipped with all the elements that moves in an orbit, forming its own new path."

\footnotetext{
${ }^{4}$ Malevich made this comment in Suprematism: 34 Drawings, published on 15 December 1920 .
} 
(Shatskikh, 2014)

\subsubsection{Sputnik}

On October 4th 1957 under the direction of Sergei Korolev the world's first artificial satellite was launched from Baikonur Cosmodrome on board a modified R7 ICBM rocket. Named " -1" (Prosteishiy Sputnik -1 [PS-1] or Simplest Satellite-1. PS-1 was not the platform for complex scientific experimentation that had originally been planned. That was called Object-D and carried a variety of experiments with a total launch mass of over $1000 \mathrm{~kg}$. It was both delayed and too heavy for the R7 rocket - then at an early stage of its development - and it was launched in 1958 as Sputnik-3. PS-1 by contrast had a limited scientific goal of successfully orbiting and being visible, which would consequently validate Tsiolokovsky's equations.

The US had also planned to launch a satellite for the International Geophysical Year (IGY) and fearing that they would launch before the Soviet Union, a much simpler 100kg satellite was hastily designed and launched. Siddiqi (2008) views the development of Sputnik as being in part driven by a dynamic of back and forth of media reports and announcements between the US and Soviet Union on their plans for the IGY from as early as 1954.

PS-1 was $84 \mathrm{~cm}$ across and made of polished titanium alloy allowing it to be visible from Earth with binoculars or the naked eye $\mathrm{e}^{5}$. It contained a simple $1 \mathrm{~W}$ radio transmitter broadcasting a pulse 3 times a second on 20 and $40 \mathrm{MHz}$. KAHN (2013) documents that the radio transmissions were specifically designed to be heard by radio hams so that the widest possible network of observations could be made both within Russia and Internationally. Radio telemetry details were published in Russian magazine Radio before launch.

The success of Sputnik caused a media and public frenzy in the West, where the press interpreted it as a demonstration of the nuclear delivery capability of the R7 rocket that launched the satellite. The mere existence of Sputnik radically re-cast the geopolitical balance of power by negating US ocean defences. Mieczkowski (2013) recounts that President Eisenhower did not however believe that Sputnik was a military threat or even that the US and USSR were engaged in a space race. In common with the Soviet Union, he underestimated the psychological and political effect of the Sputnik launch. The Sputnik crisis was cast in terms of cultural competition in the media and caused serious political problems for Eisenhower

\footnotetext{
${ }^{5}$ This ability to be seen directly with the naked eye was exploited in early US satellites, Echo and Pageos series, which were vast 30m Mylar inflatable spheres used as light and radio reflectors.
} 
that would eventually lead to the creation of both the Defense Advanced Research Projects Agency (DARPA) and the National Aeronautics and Space Agency (NASA).

It is clear that rather than being an explicitly military or scientific satellite, the world's first artificial satellite was designed primarily as a cultural object, and one with the widest possible global audience both in visible spectrum and in the most widely used amateur radio bands. At the time US Commissioner of Education, Lawrence Derthick commented "The game is economic and cultural conquest of the world." (Mieczkowski, 2013, p.65)

In 1957 the USSR and the US were engaged in what Saunders (2000) terms the cultural cold war in which many aspects of culture were co-opted by both parties for the purposes of geopolitical propaganda. In the USSR the arts had been state sanctioned and controlled since Stalin's time, and socialist realist art of the period was explicitly anti-western, antibourgeois, and in support of the state (Grorss, 2008). In the US, abstract expressionists including Pollock were seen by the CIA to produce art that was an emblematically individualist rejection of Socialist realism. Touring exhibitions were then clandestinely funded by the CIA, and the New American painting show toured Europe throughout 1958/9 (Museum of Modern Art, 1959). In contrast the USSR chose Sputnik as the centre piece of its 1958 Brussels World Expo pavilion as a pinnacle of Soviet collective technological and cultural achievement.

The exhibit was created at the proposal of cosmist and science fiction author Vasilii Dmitrievich Zakharchenko who also edited a publication called Sputnik Siegelbaum (2012). In this Sputnik was anthropomorphised and the simple non-scientific message of Sputnik was emphasised.

\footnotetext{
'Beep Beep: Any newborn tells the world of its existence with a cry, I conformed to this tradition and hardly had I appeared, when I cried "beep-beep". The entire planet heard my voice. What is the secret of my popularity? I serve humanity and progress ... . Let's drop the formalities. You can call me Beep Beep.' Zakharechenko (Siegelbaum, 2012, p.129)
}

During the cultural cold war the individualist message of Jackson Pollock's abstract expressionist paintings directly competed with the simple aesthetic of the 'Beep Beep' message of Sputnik. In this cultural contest the two works function as both artwork and propaganda. Pollock's paintings are clearly visual artworks, unwittingly co-opted as propaganda. Sputnik on the other hand was propaganda, taking the form of media artwork. 


\subsubsection{Post Sputnik}

The following works in the post Sputnik era mark a transition to intentionally produced artworks for the space environment, that each involve a shift of viewpoint. (McLuhan, 1970) extends his core concept - the medium is the message - in an electronic version of a Copernican revolution, argues that the satellite completely re-configures humankind's vision of itself and its place in the universe. "With the satellite, for example, going around the planet, the planet becomes an incidental content of satellite environment, a man-made satellite environment, which completely alters the planet and us on the planet."(McLuhan, 1970)

The transformation of world view described by Mchluan was given pictorial form in The Blue Marble image - a view of the whole disk of Earth from space. It was taken by the one of the crew of Apollo 17 (probably Schmitt) in 1972 on their journey to the Moon, and was only possible in a single frame at the great distance of 29,000 km from Earth. In terms of access to space, the creation of this image was only possible for a very small group of 24 astronauts - privileged potential photographers - who were all public employees of NASA rather than professional private artists. It was preceded by Stewart Brand's 1966 badge campaign "Why haven't we seen a picture of the Whole Earth Yet?" and became a potent symbol of the environmental movement and the new universalism, and marked a turning point in humankind's image of it's position in the Universe (Diederichsen \& Franke, 2013). 


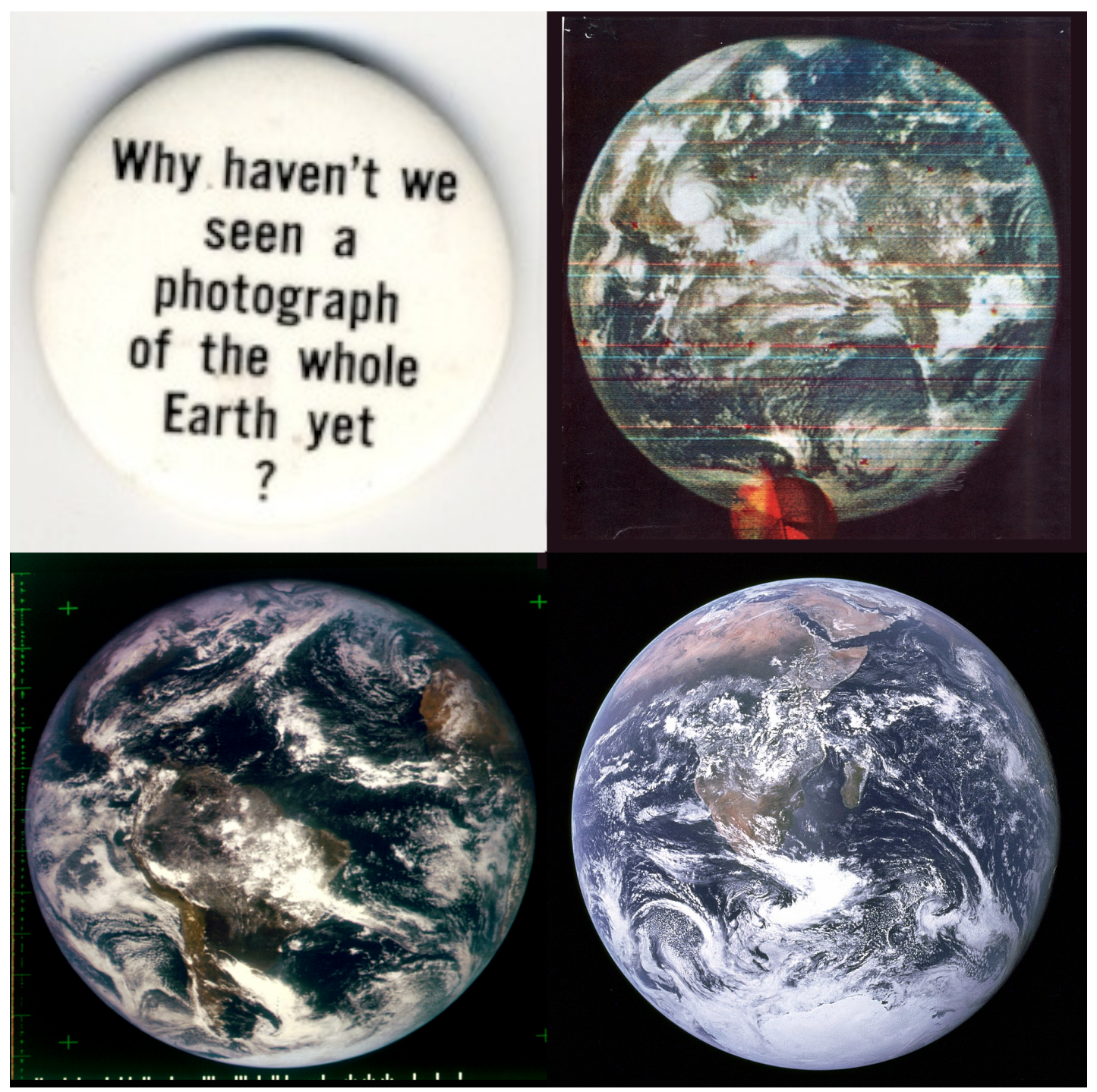

Figure 2: Whole Disk Images, Brand, DODGE, ATS-3, Apollo 17

Note. Collage of Whole Disk Images from 4 images.

1. Why haven't we seen a photograph of the whole Earth yet? Campaign button 1967, By Stewart Brand (Brand, n.d.). With permission.

2. DODGE satellite image. 3. By Department of Defense (1967). Public Domain.

3. ATS-3 image. By NASA (1967). Public Domain.

4. Apollo 17:Blue Marble photograph taken en route to the Moon by Apollo 17 crew. By NASA (1972). Public Domain.

The views of the Earth from the journey to the moon and the image of Earth rise over the moon, were realisations of the view described by Lucian in the Icaromenippus in 190 A.D. 
It was not the first whole disk Earth image - these were composite scans taken in 1967 from satellites DODGE and ATS-3. An image from the later was used on the 1968 cover of the Whole Earth Catalog that was published by Brand. The DODGE image was the first colour whole disk image of the Earth, and was created as incidental content by a satellite going around the planet as McLuhan described.

The blue planet was referenced in Soviet commentary of the 1975 Apollo-Soyuz rendezvous which marked the beginning of an era of peaceful international co-operation in Space that continues today (Ross-Nazzal, 2010). As the US and Soviet astronauts shook hands in orbit, the commentator evoked the symbolic unity of the blue planet. "Dear American TV people it would be wrong to ask which country's more beautiful. It would be right to say there is nothing more beautiful than our blue planet." Kubasov in (Ezell \& Ezell, 1978)

Professional artists were involved in Space Art projects that accompanied the Apollo programme. A high profile group of Rauschenberg, Novros, Chamberlin, Oldenberg, Myers and Warhol collaborated on Moon Museum, a small engraved ceramic chip the size of a sim card for Apollo 12. (Guridi, 2019, p.244) describes how after a failed direct approach to NASA through the Metropolitan Museum of Art a sympathetic anonymous engineer purportedly attached the chip to the leg of the lunar lander and left on the Moon in 1969, although there is no supporting photographic documentation.

Another small sculpture 'Fallen Astronaut' by Paul Van Hoeydonk was placed on the moon secretly by astronaut David Scott in 1971 along with a plaque honouring astronauts who died in service. This work was photographed and verified although similarly controversial. In terms of access to space these two works were not commissioned by NASA and were installed using clandestine means with help from NASA staff.

In 1970 Frank Malina made a call for a more formal role for the visual arts in the space programme. Malina was an aeronautic engineer, kinetic artist, and co-founder of the Jet Propulsion Laboratory (JPL), International Association of Astronomical Artists (IAAA) and founder of the Leonardo art science organisation. As well as more traditional forms Malina suggested that the unique environment of space could lead to more experimental works. "It seems to me that the results already obtained at this stage of man's entry into space deserve more consideration by artists working in the field of the visual fine art". (F. J. Malina, 1970)

The 1972 and 1973 plaques which left the solar system on the Pioneer 10 and 11 spacecraft were anodized aluminium etched with a diagram of humans and their place in the solar 
system. They can be considered space artworks and were a successful overt collaboration between NASA, Burgess, Sagan and Drake. The rationale was to create a non-linguistic communication with a potential intelligent extra-terrestrial audience outside of the solar system.. The detailed paper by Sagan, Sagan and Drake (1972) focuses on portraying humanity in a literal way and is a first attempt at a truly galactic artwork. "This message then is a first attempt to specify our position in the Galaxy our epoch and something of our nature." (Sagan, Sagan, \& Drake, 1972)

Nam June Paik's work Wrap around the World was Earth bound but used a network of broadcast satellites to create TV transmissions from ten countries to over 50 million viewers (Paik, 1984). In an interview with Eduardo Kac, Paik prefigured future art satellites and said: "This is my last satellite show, but it is also the beginning of a larger satellite movement of the future."(Kac, 1988) 


\subsection{Art Satellites}

\subsubsection{Overview}

This section consists of a survey of the field of dedicated art satellites, both proposed, unlaunched and launched. Only autonomous satellites that are designed to fly freely outside of other spacecraft are included here for the sake of brevity. The list is largely based on a satellite art survey by A. Bureaud Annick (2015), updated to reflect some recent developments. An episode concerning regulation of space art is included with the relevant artwork.

\subsubsection{Unlaunched Art Satellites (as of 2020)}

This chapter of Space exploration was characterised by the development of the International Space Station and the regular service of the Space Shuttle ferrying experiments and passengers backwards and forwards to Low Earth Orbit. During this period, access to space began to be routine and the commercial satellite business developed. The improvement in access and regularity of service resulted in the development of a range of speculative art satellite proposals.

\begin{tabular}{lrll}
\hline Satellite & Proposal Date & Status & Artist \\
\hline Beacon, Star-Cloud, Man-Star, Earthlog & 1971 & Idea & Albert Notarbartolo \\
Space Flight Dolphin & 1982 & Prototype & Richard Clar \\
OURS & 1984 & Prototype & Arthur Woods \\
OURS-SPS & 1988 & Prototype & Arthur Woods \\
La Roue Céleste & 1984 & Feasibility & Jean-Marc Phillipe \\
Concurs "La Tour Eiffel" 12 projects & $1986-87$ & Idea & Various \\
ARSAT (Eiffel Competition) & $1981-83$ & Feasibility & Pierre Comte \\
ARSAT (Eiffel Competition) & $1986-87$ & Feasibility & Pierre Comte \\
The Space Chronometer (Eiffel Competition) & $1986-87$ & Proposal & Chris Coles and Alan Jefferson \\
The Goodwill Constellation & 1989 & Feasibility & James Pridgeon \\
Keo & 1992 & Feasibility & Jean-Marc Phillipe \\
ARTJOM-MM & 2001 & Prototype & Dragan Živadinov, Marko Pelijhan \\
DUAL S17 & 2001 & Prototype & Dragan Živadinov, Marko Pelijhan \\
LADSAT & 2001 & Proposal & Dragan Živadinov, Marko Pelijhan \\
Gregnik & 1996 & Prototype & Gregory Green \\
Mur-Sat & 2010 & Prototype & Jogi and Reni Hofmüller, \\
& & & Christian Pointner, et al.. \\
Ulises 1 & 2010 & Baloon 2015 & Juan José Diaz Infante \& \\
Non Functioning Satellites & & & Colectivo Espacial Mexicano \\
Ulisese 2.0 & 2013 & Prototype & Trevor Paglen \\
& 2015 & Prototype & Juan José Diaz Infante \& \\
\hline & & & Colectivo Espacial Mexica \\
\hline
\end{tabular}

Table 1: Unlaunched Art Satellites

(A. Bureaud Annick, 2015) 


\subsubsection{Arthur Woods}

Arthur Woods' OUR which later became Space Peace Star was a proposal for giant inflatable visible satellite in the shape of a circle in the sky. In 1993 Woods sent his Cosmic Dancer sculpture to the Mir space station, and in 1995 organised an exhibition of 20 artists work on Mir, Ars Ad Astra (Woods, 2020).

\subsubsection{G.A.S programme}

The Space Shuttle offered artists direct access to the space environment through NASA's 'Get Away Special' programme in which artworks could be designed to fit inside a special canister in the Space Shuttle's hold. McShane's S.P.A.C.E (1984) and Kurtz's 'Vertical Horizons' (1986) both made use of this programme. In Jan 1986 the Shuttle Challenger exploded just after launch with the loss of all on board and there was a general retreat from more speculative projects, the G.A.S programme was ended, and no autonomous art satellites were ever launched from the space shuttle (Woods, 2019).

\subsubsection{Eiffel Tower Competition}

In June of 1986 The Eiffel Tower Competition was launched to commemorate the centenary of the Eiffel Tower. They asked artists and innovators to design an orbital artwork that would be visible from the ground by the naked eye. 12 were shortlisted and the winner was 'L'anneau Lumiere' by Groupe Spiral, a 24 kilometer circumference ring of reflective inflatable plastic. There were serious public objections from astronomers and other concerned scientists and the project was halted after complaints.

Space artist Chris Coles writing about his own finalist entry for the competition clearly saw the dispute as in issue of territory, jurisdiction and funding. "The tragedy is that the very people who have illustrated the immense challenge of space should be those who then 'harm science' in their bid to stop anything or anyone from impinging on their 'territory'." (Coles \& Jefferson, 1989)

\subsubsection{Space Art Regulation}

Murdin (1991) in an article entitled Threat to Astronomy by Space Art presented a detailed analysis of satellite light interference in The International Astronomical Union (IAU) Colloquium proceedings and identified three categories of visible display satellites as especially 
problematic. These were Art Satellites with purely artistic goals, Commemorative Satellites that celebrated events or anniversaries and Advertising Satellites that promoted commercial ventures. He proposed a code for 'Control of Art Satellites' by agreement between national space administrations that would limit maximum duration, focusing of light, angular extent and orbital visibility. The goal was to make visible art satellites impossible. "If the display is so prominent that it is worth being called space art, it is probably too prominent for astronomers to accept." (Murdin, 1991)

It is clear that under these guidelines both Sputnik, Echo and PAGEOS programmes would never have been realised.

Roger Malina gave a spirited defence of Space Art in the same IAU proceedings journal and highlighted research that showed that $30 \%$ of scientists believed that Space Art was frivolous (R. F. Malina, 1991/ed, pp. 145-152). He usefully analysed the impact of various categories of space art in terms of its impact on scientific research and highlighted the international treaties that governed space as well as early artist attempts to self legislate.

The Sky Art Manifesto mirrored some of the more transcendental aims of the Cosmists while calling for productive alliances with all space agencies.

Our release from gravity represents a fundamental shift in human consciousness - flight and release which open a new dimension of humanity....Sky artists enthusiastically seek productive alliances with all space agencies. We are asking for the establishment of national and international councils that will advocate specific artistic projects to the appropriate institutions and agencies. Sky Art Manifesto (Goldring, 1987)

Neither the Control of Art Satellites code nor the Sky Art Manifesto resulted in the creation of significant multi-lateral treaties or agreements relating solely to Space Art, and Space Art continued to be reliant on the existing legislative environment and participation of individual Space Agencies which continued to controlled all launch access, for both commercial and non-commercial payloads.

\subsubsection{M.I.R}

The break up of the Soviet Union in 1991 and the economic shifts that followed it, led to the commercialisation of the Soviet space programme during the 1990s and it's split up into separate space programmes in Ukraine and Kazakhstan. This resulted in both technology 
transfer to other nations, and a range of commercial launch operators which increased opportunities for artists to directly access space technology. An early example is the Microgravity Interdisciplinary Research project which between 2002-2004 used the zero gravity Illyushin-76 free fall plane of the cosmonaut training facility to offer a group of artists the opportunity to create works in micro-gravity (Triscott \& La Frenais, 2005).

\subsubsection{Ulises}

Access to space technology continues to be problematic and highly regulated even at a component level. Space technology has for instance long been governed by US Defence Department export restrictions, technology transfer restrictions and overseas personnel restrictions. This has impacted space artists working outside of the US. The Mexican Space Collective project Ulises in 2010 had to apply for specialist export licences to get supplies of particular solar panels for their prototype satellite. (Infante, Finn, Castro, Gasca, \& Lafuente, 2017)

I soon learned that I would have to apply for special permission from the U.S. Department of Defense in order to see the project out. Unbelievable: I had to submit a mission manifest to them. Unbelievable: I had to take an official oath, swearing that 'I won't export my payload to an enemy of the United States.' (The payload I had planned on launching? Poetry.)Infante (2018)

Ulises was one of two projects which designed, prototyped, built and tested functional art satellites around 2010 which have not to date been launched into orbit. The other is the Austrian Arts Group Mur.at art satellite platform. Both of these projects were based on the Tubesat form factor which is a competing nano satellite form factor to Cubesat, and are still manifested for launch on rocket startup Interorbital Systems own platform. While Interorbital Systems have had some success in launching sounding rockets up to $10 \mathrm{~km}$, they have not yet achieved orbital flight beyond the Karman Line and are currently launching small satellites through other operators. For the two art projects the loses in launch investment and time have been critical and while Ulises continues to test their satellites with stratospheric balloon launches, neither project has deployed in orbit to date. 
7.2.9 Launched Art Satellites (as of 2020)

\begin{tabular}{lllllllll}
\hline Satellite & Launch Date & Re-entry Date & Deployment & Visible & Radio & Launch & Form Factor & Description \\
\hline Sputnik & $<1957-10-04$ Fri $>$ & $<1958-01-04$ Sat $>$ & Deployed & Yes & 20MHz, 40MHz & CCCP & Custom & propoganda \\
\hline OSSI-1 & $<2013-04-19$ Fri $>$ & $<2013-06-30$ Sun $>$ & Deployed & No & Failed & NovaNano/Roscosmos & 1U Cubesat & art \\
Artsat Invader & $<2014-02-28$ Fri $>$ & $<2014-09-02$ Tue $>$ & Deployed & No & $437.325 \mathrm{MHz}$ & Mitsubishi/JAXA & 1U Cubesat & art \\
TWOi & $<2014-04-19$ Sa $>$ & $<2014-05-14$ Wed $>$ & Failed & No & Failed & Space-X/NASA & chipsat & art \\
TWOi 2.0 & $<2018-11-17$ Sat $>$ & $<2019-04-02$ Tue $>$ & Deployed & No & $437.240 \mathrm{MHz}$ & NorthrupGrumman/NASA & chipsat & art \\
Orbital Reflector & $<2018-12-03$ Mon $>$ & $\sim 2021$ & Failed & No & Telemetry & Space-X & 3 U Cubesat & art \\
ENOCH & $<2018-12-03$ Mon $>$ & $\sim 2025$ & Deployed & No & Passive & Space-X & 3U Cubesat & art \\
\hline Humanity Star & $<2018-01-21$ Sun $>$ & $<2018-03-22$ Thu $>$ & Deployed & Yes & Passive & Rocketlab & custom & commercial \\
Red Tesla ${ }^{6}$ & $<2018-04-07$ Sat $>$ & $\sim$ & Deployed & No & Telemetry & Space-X & car & commercial \\
\hline
\end{tabular}

Table 2: Launched Art Satellites 


\subsubsection{OSSI-1}

It was from the Baikonaur Cosmodrome in 2013 that Korean artist Hojun Song launched his OSSI-1 satellite into Low Earth Orbit on a Roscosmos Soyuz-2-1a rocket (Gardiner, 2010). The launch was a commercial ride share with other Cubesats that accompanied Roscosmos' Bion-M No.1 live animal experiment. The Open Source Satellite Initiative was Song's 7 year project to design, build and launch a personal satellite built by himself from off the shelf components. The project itself and the satellite can be considered as an artwork, and Song's process sits within a tradition of interdisciplinary D.I.Y and open source art practice (Kim, 2014). Song set out to answer the question of whether it was possible for an individual to build and launch a satellite, and this proof of access represents a key transition in satellite art. The enabling aspects of the project were the commercial access to space, ride sharing and the new $1 U$ Cubesat form factor. The major expense in the project was the launch itself which cost $\$ 100,000$ USD and raising this substantial figure was problematic for the project, with innovative fund raising attempts including T-shirt sales. The other major issue they faced was radio licensing from the ITU, which meant that the launch was brought under the umbrella of French company NovoNano in order to gain regulatory approval. In 2011 speaking at the signing of the launch agreement with NovoNano, Hujon Song wore his trademark ceremonial sash embroidered with the text Science is Fantasy and said:

Sputnik went to space in 1957 and even a dog went to space too. I think it is really natural to think that by 2011 that as an individual we start our own space programme and this will be the first individual satellite made. (Song, 2011, June 11)

The launch went ahead as scheduled and the satellite was successfully deployed but no transmission was ever received from the satellite. The developers speculated that the TLE (tracking co-ordinates) was incorrect.

Nevertheless the launch was a major milestone in the history of art satellites coming as it did 55 years after Sputnik and which was also launched from Baikonaur Cosmodrome. Not only does OSSI-1 represent the first personal satellite in space, but also the first dedicated autonomous art satellite. Sputnik can of course alternatively be considered as the first art satellite, but it's mixed status as propaganda and orbital experiment problematise this categorisation. 


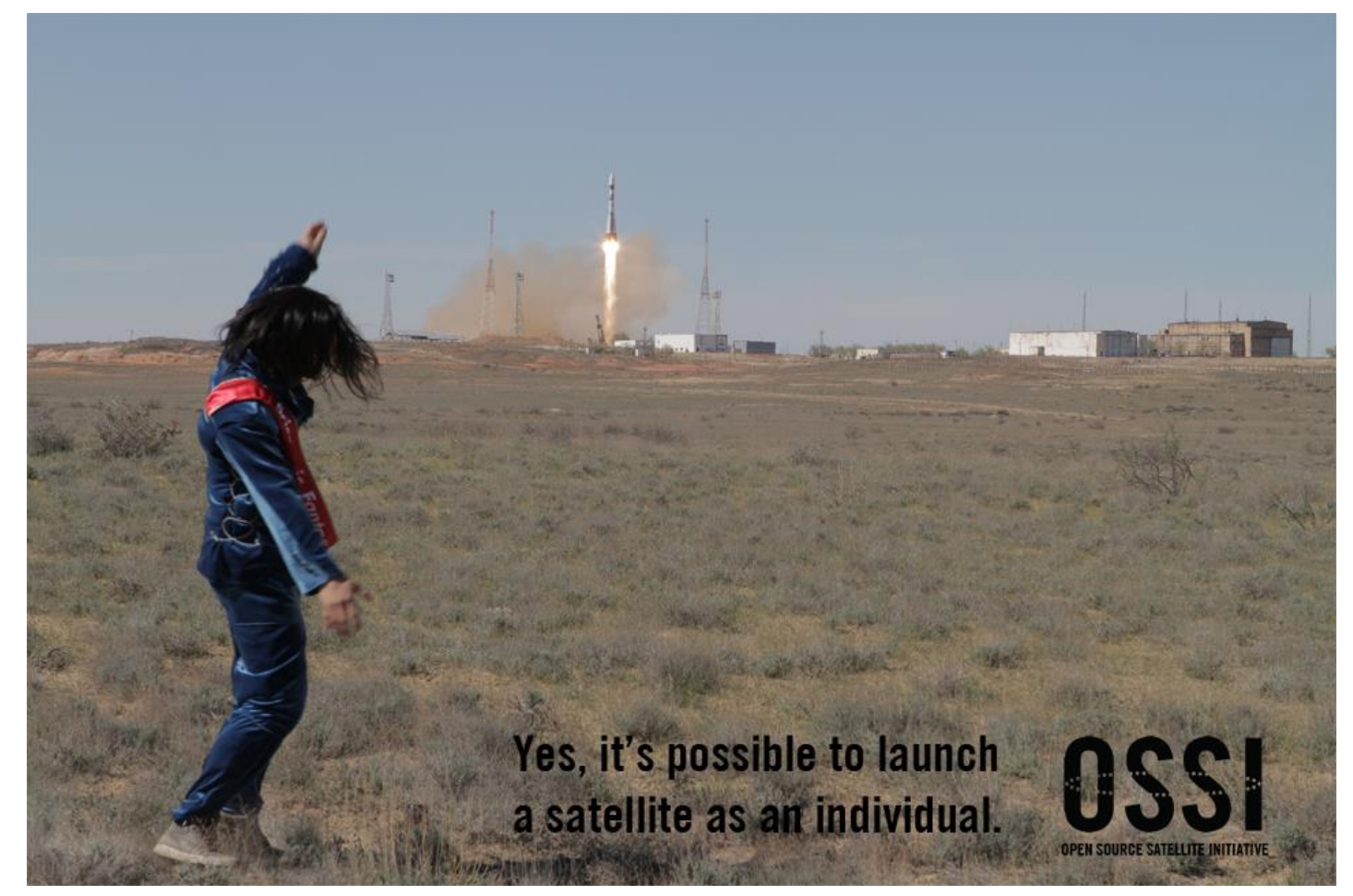

Figure 3: OSSI-1 Launch

Note. Image of OSSI-1 launch from Baikonaour. By Song Creative Commons AttributionShareAlike 3.0 unported

"OSSI-1 satellite is Launched on 19th April 2013. It was all possible but a bit expensive." (Song, 2014a, February 22)

\subsubsection{Artsat1: INVADER(*)}

ARTSAT1: INVADER(*) was a Cubesat built in a collaboration between University of Tokyo and Tama art University and launched in 2014 and it laid claim to being the world's first art satellite ArtSat (Artsat, 2011). The satellite extended work started in Japan where the Cubesat form factor was initially developed and was launched on a Mitsubishi Heavy Industries H11A rocket by Japan Aerospace Exploration Agency (JAXA).

INVADER had an open interface and number of systems for tone generation and transmission to Earth that could be utilised by artists and in this way the satellite was conceived as a platform or infrastructure for artists to make satellite based works. On June 28, 2014 Akihiro Kubota and Tomomi Adachi performed the work Cosmic Poem which was generative poetry created by software and transmitted by the satellite as it made passes over various areas of the Earth (Amsat, 2014, June 26). Signals from work could be received using amateur ra- 
dio equipment and performances were scheduled to coincide with orbital passes which were heard at various public gatherings and mirrored the amateur radio transmission technique of Sputnik. This mirrors Paik's use of satellite transmission in Wrap around the world but this time on what can be considered artist infrastructure that can be considered artist run space.

\subsubsection{Humanity Star}

Humanity Star was a personal project of Rocketlab’s founder and CEO Peter Beck. Rocketlab is a recent private rocket launch company started in New Zealand and now with offices in the US and launch facilities on the Mahia Peninsular in New Zealand and Wallops Flight facility in Virginia USA. During the second test flight of their new Electron rocket, Beck included a Im 76 sided reflective carbon fiber polyhedron called Humanity Star along with 3 Cubesats. The payload tested the ability of the rocket to inject satellites into orbit but the Humanity Star was presented by Beck as an artwork.

The Humanity Star was designed to be a temporary symbol in the night sky that encouraged everyone to look up, ponder humanity's place in the universe and think about how we can work together as one species to solve the challenges facing us all. (Beck, 2018)

Humanity Star was designed to flash and be visible with the naked eye and the work caused controversy especially from astronomers. It was also considered by some to be advertising for the Rocketlab, described by others as space graffiti, and again was raised by astronomers who largely mirroring Murdin's 1991 concerns. The work certainly had a dual purpose in advertising the new capability of Rocketlab as a company, and New Zealand as a new space faring nation. In interview however Beck revealed that he had been planning the work for many years before the existence of Rocketlab and stood by it as primarily an honest cultural statement.

\subsubsection{Red Tesla and Starlink}

Days after Humanity Star's launch on June 2, 2018 Elon Musk launched a Tesla roadster into orbit around the sun as a test payload for the Falcon Heavy test flight generating significant publicity. Comparison's were inevitably drawn and most considered the Tesla as a purely commercial publicity stunt peppered with popular culture references, rather than as an artwork, and this appears to have been more appreciated by the general public. Gorman and 
Roberts (2019) it as primarily symbolic communication that ushers in a new era in which wealth structures space exploration. The two launches were only possible because of the control and privileged access that Beck and Musk to spare capacity in their own rocket companies. Diaz (2018) describes the new generation of private space operators, that includes Elon Musk, Jeff Bezos and Richard Branson as NewSpacers and points to double standards in the transition from public to private. "On the one hand, NewSpacers push for a libertarian deregulation of outer space, as a zone for untrammelled profits; on the other, they are heavily reliant on government-built infrastructure and NASA contracts." (Diaz, 2018)

In May 2019 Space-X began launching the Starlink satellite constellation with an initial planned deployment of 1584 satellites which will rise to potentially tens of thousands as the network grows (Starlink, 2020). The satellites are visible and far more damaging to astronomy that any of the previous visible satellites. While mitigation strategies, coatings and sunshades are being developed, this is being done after the initial launch and that points to a fundamental shift in power relations between the time of the cancelled Eiffel competition and now. Here I put forward that when a few national space agencies controlled launch vehicles, they also had de-facto control of what was launched. Now that private commercial operators have launch capability and hence de-facto control, nations and the scientific lobby have lost direct control, and now power relations are becoming indirect shifting to regulatory bodies like the FCC, contract awards and other means.

\subsubsection{Orbital Reflector}

The last two art satellites considered here are Trevor Paglen's Orbital Reflector, and Travares Strachan's ENOCH which were both Cubesat based satellites launched on the same Space-X Falcon-9 in 2018. Paglen's work was the more ambitious of the two and comprised a large faceted flashing inflatable structure reminiscent of the Echo and PAGEOS satellites but with similar functionality to Humanity Star. Paglen is a well regarded, critical and prolific artist and he was commissioned by the Nevada Art Museum and a range of sponsors to create a work following his 'Non-Functional Satellites' with a budget of over $\$ 600,000$ USD. The launch was successful but a combination of unanticipated events conspired to prevent the sculpture's inflation. Firstly the US Air Force (USAF) was unable to distinguish between and assign tracking numbers to the satellite cluster when it was deployed, and this prevented the FCC from being able to immediately approve the inflation. At this point there was an 
unplanned US government shutdown which lasted 35 days, during which time there was no communication with the FCC. By the time the USAF and FCC were in a position to process the approval, communication had been lost with the satellite and the mission could not be completed. Here the already complex and remote orbital environment was exacerbated by a failure of governance, regulation and ultimately political process.

\subsubsection{ENOCH}

Artist Travares Strachan's ENOCH Strachan (2017) by contrast is a passive sculptural object and remains in orbit as intended (as of 2020). It consists of a 3U Cubesat frame holding a 24 carat gold canopic jar topped with a bust of Robert Lawrence. Lawrence was the first African American astronaut selected for a national space programme and who died in 1967 while training a junior pilot. The work honours his legacy and the title Enoch refers to a biblical figure who ascended directly to the afterlife. As such the work both echos themes of immortality present in early Russian Cosmism and is also an example of a commemorative work as described by Murdin. Strachan here continues a theme from his work Orthostatic Tolerance (2006-2010) in which he subjected his own body to rigorous astronaut training at the Gagarin Centre in Star City, Russia (Díaz, n.d.). Further he continues a theme from other works about invisibility, the practical invisibility of the artwork itself foregrounding the historical invisibility of Robert Lawrence. In terms of institutional arrangements, the work was created in association with Los Angeles County Museum of Art (LACMA) in the LACMA Art + Technology Lab whose founding sponsor is Space-X, and so this work is a civic commissioned, privately funded, privately launched artwork, with no radio and hence no operating license, and is thus completely created under the conditions of New Space industry. 


\section{Case Study}

\subsection{Overview}

The Weight of Information (TWOi) was a satellite based artwork and art-science research project that started conceptual development in 2009, had a failed deployment in orbit in 2014, and concluded with a successful autonomous orbit in March 2019. This case study explores some aspects of the process by which it was created and attempts to draw out relevant themes. The case study is divided into four situations, each of which explores an aspect of the project and has its own reading and conceptual framework that responds to the specifics of each situation. The case study does not aim to be comprehensive documentation of the TWOi project, though it does place each situation within a timeline that encompasses the whole project. While the case study does not aim to directly engage with the research topics explored in the artwork, a very brief outline of the project is included here for the sake of orientation.

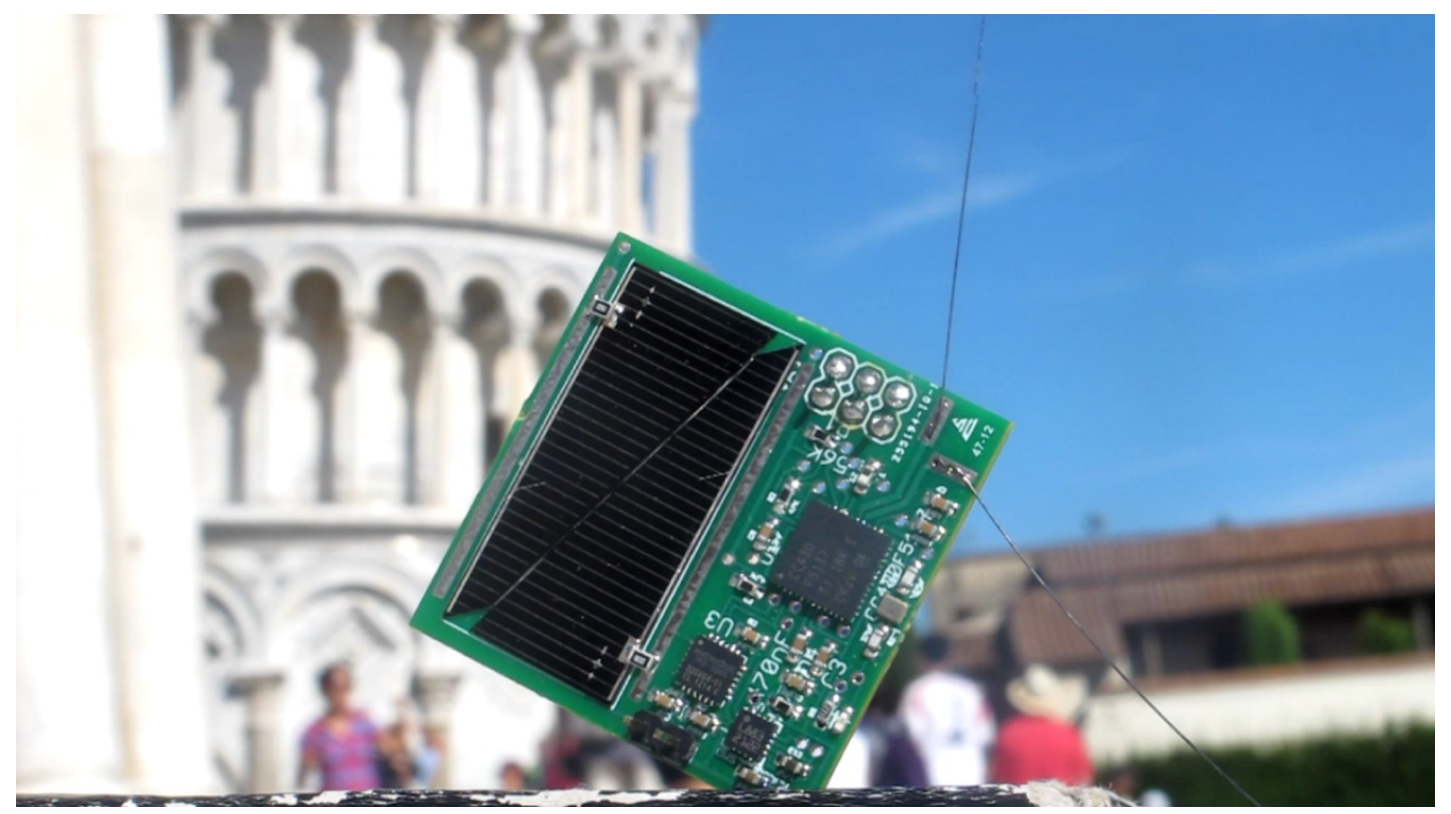

Figure 4: TWOi Satellite

Note. TWOi Satellite. By Julian Priest.

TWOi was originally an idea for an artwork and research project that explored the relation between gravitation and information. Through an opportunity to create a satellite project, this idea was developed into a two part artwork and experiment, with a satellite orbiting in low earth orbit, and a series of Earth based public events. The enabling satellite project was 
the open-source crowd funded KickSat project and this project formed the key enabling relationship of the development. The artwork proposed an imaginary universe or storyworld in which information and not mass is responsible for gravitation. The satellite was to be placed in a decaying orbit and would burn up on re-entry. Characterised as a tragic hero, the satellite attempted to escape its fiery destiny by performing an experiment in which it collected data and immediately deleted it in order to ascend by forgetting. Meanwhile, on Earth, participants in public events were asked to delete their own personal information to make the world a little lighter and aid the satellite in its escape. The artwork addresses our relationship to the increasing role of information and data in society, and poses the question of whether it is truly possible to delete information and hence have agency over it. As part of the artwork a speculative physics experiment was performed to test the theory that information causes gravitation. The project covered a decade from ideation to completion, a large number of events, and two attempts at launch. The first attempt in 2014 did not result in a deployment of the first TWOi satellite, and the project was completed in 2019 with the successful orbit of the TWOi 2.0 satellite.

The first situation looks in detail at the development process of TWOi within the larger KickSat project which was framed as providing a personal satellite. This underlying idea of the personal spacecraft is interrogated and contrasted with the detail of the development process. An alternative reading of the project is developed which foregrounds the specific features of the development environment and the infrastructure of the Kicksat project. This project infrastructure is examined with reference to Brattain's concept of interface, Kong's relation of the user to the interface and the concept of boundary object drawn from Leigh Star.

The second situation looks at the evolution of the first 2014 launch from announcement to eventual re-entry burn-up, that was caused by a malfunction in orbit. This situation is analysed with reference to Gell's concept of oeuvre and Husserl's phenomenology of time. The ideas of contingency and dependency are investigated with reference to Hodder and finally compared to Barad's agential realism. These last two are related through similar concepts of entanglement.

The third situation examines a delay in the project in 2015-2016 that was caused by regulatory difficulties relating to radio spectrum and debris. This is contextualised within the changing public/private relationships in New Space access relations and the evolution of 
existing spectrum regulating bodies.

The fourth situation describes aesthetic techniques used to address the extreme physical distancing entailed in orbit as a location for a cultural projects, design or space art. The use of the narrative device of tragedy in bridging the physical distance and creating an emotional correspondence is developed and contrasted with the use of line.

\subsection{Material}

This thesis is retrospective and field notes and interviews specifically on the processes examined here were not taken directly during the time of the project. A standard ethnographic project relies on interviews, observations and documents to create a body of data and a narrative is then constructed from that. What can be considered the primary field work material here therefore consists in what could be considered ephemera from that was produced by the project as well as associated third party media and material drawn from other organisations that were involved directly or peripherally in the project.

For documents there are email correspondence with the author, archives of mailing lists, software repositories and websites that were used in the development of the projects. The observations are direct recollections detailed by the author as autobiographical incidents as well as reflections of an auto-ethnographic nature. There are no direct interviews in the source material, though to some extent publicly broadcast video recordings or online conversations fulfill this role. All of the original material presented here is freely available on the internet and is treated as a matter of public record with the exception of private email correspondence. Permission to publish private email correspondence is sought in the small number of cases where it is quoted directly and the author is the collocutor in each of these cases.

Throughout the situations a distinguish is made when necessary between various aspects of TWOi and these are termed TWOi-project, TWOi-artwork, TWOi-character, TWOi-narrative and TWOi-satellite. TWOi-project is the overall project and includes all aspects, TWOi-artwork is the publicly presented event and installation group. TWOi-character is a fictional character informally invoked when giving explanatory framing descriptions of the TWOi-artwork. TWOi-storyworld is the descriptive narrative with TWOi-character as protagonist. TWOisatellite is the physical satellite which is identified as TWOi-character. 


\subsection{Situation 1}

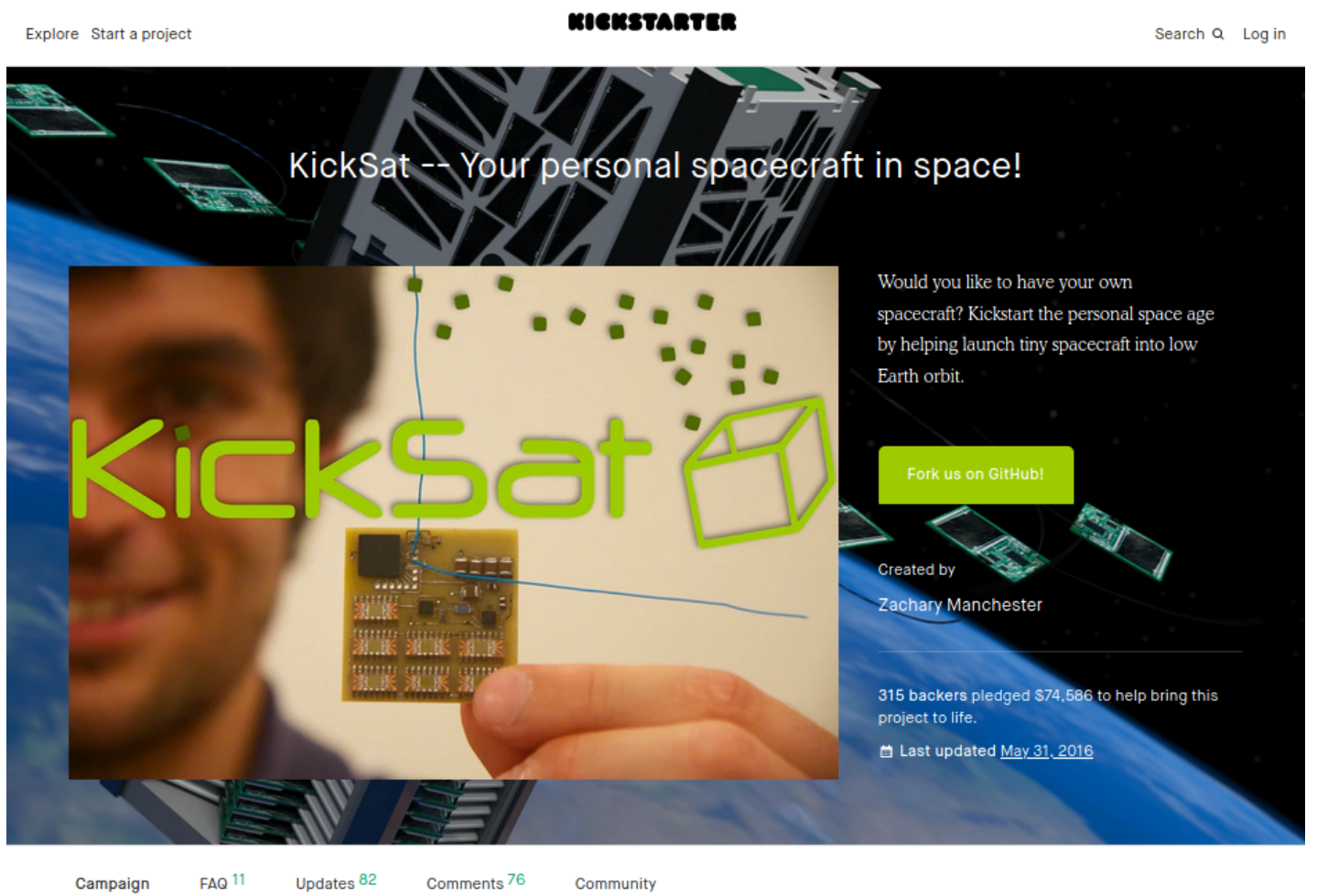

Figure 5: Kicksat Kickstarter Page

Note. Screenshot of Kicksat Kickstarter web page. By (Manchester, 2011, October 4). With permission of Zac Manchester.

On 16th November 2011 I received an email from an ex-NASA rocket engineer friend with a link to the new Kicksat programme. It was an ultra small form factor, open source, personal satellite project that was offering a launch of a standardised satellite for a Kickstarter pledge of \$300 USD or \$1000 USD for the developer version.

As soon as I saw the page, and with no firm idea of a project, I instantly signed up, entered my credit card details and became a supporter. The Kickstarter campaign goal of $\$ 30,000$ was easily exceeded with a total of $\$ 74,586$ and 315 backers, the campaign closed on <2011-12-03 Sat $>$ and the project went ahead.

The satellite that was used as material for the TWOi-artwork was provided by a project called KickSat. In this section I describe my experience of the Kicksat development process from initial on-boarding into the Kicksat project, up until the delivery of the custom code that was flown on the TWOi-satellite. This description is based on personal reflection 
of the experience of developing a project for the Kicksat platform. The KickSat project was an open-source and crowd-funded satellite development project that offered developers the chance to own a personal spacecraft. The idea of a personal satellite is examined with reference to the detail of the development process. The previous barriers to personal satellite ownership of cost and administrative access are explored in the context of falling launch costs, form factor standardisation and ride sharing. The Cubesat form factor is used as an example and is compared with the novel Kicksat approach. The Kicksat project made use of several external services in the creation and delivery of the project, including Kickstarter and GitHub. The use of these services and the underlying methods of crowd funding and open source are explored. An alternative reading of these services as boundary objects is explored and contrasted with the idea of the personal satellite.

\subsubsection{Personal Spacecraft}

The strap line of the KickSat project "Your Personal Spacecraft in space!" promises to realise the dream of personal spacecraft ownership. This resonates in particular with a generation of adults that grew up within the cultural environment of the Space Race.

Lemonnier (2012) theorizes a similar dynamic between childhood experience and adult action in an examination of his own personal passion for racing cars and drivers of the 1950's. He describes how the cultures surrounding his 1950's generation's youthful collection of scale models was expressed in later life in classic car collection and racing. He places the models and classic cars as objects at the centre of this material culture.

I posit that in the case of such pieces of material culture too, the multiplicity, diversity, and time-depth of the material actions related to the objects in question is a crucial dimension of their ability to bring people to act together. (Lemonnier, 2012)

Reflecting on my own personal multi-sited experience of Space as a child in the 1970's, I remember witnessing events such as the Apollo/Soyuz hookup live on TV, and then later seeing space travel become common place with the Space Shuttle. In this Cold War period media about Space continued to be heavily politicised and missions had strong political goals and supporting public imagery. The Apollo/Soyuz link up, for instance, was the outcome of a policy of détente and co-operation between the US and USSR in Space that became a treaty 
in $1972^{7}$. It resulted in a handshake in orbit between astronauts from each nation that was broadcast for public consumption on TV in 1975. ${ }^{8}$ These media events were reflected in a huge range of space related media of the 1970s; science fiction literature, TV series such as Star Trek, Blake 7, films like Star Wars and Battlestar Galactica, in music David Bowie's Space Oddity, as well as supporting material objects such as Star Wars card and models. There were space themed role playing games such as Traveller and a large range of arcade games, with titles such as Lunar Lander, Space Invaders, Defender and Scramble available in public venues or on living room consoles. Some computer games for early home computers like Elite were sold, others such as Space Invaders in 1K on the ZX81 were typed in by hand. The Space themed childhood cultural environment of the 1970s created a world that blurred media of actual events, imaginary worlds, interactive and participatory game play and programming. During the 1970s any kind of actual access to space technology by private individuals was a practical impossibility. Perhaps the combination of the centrality of space to the public narrative of the cold war combined with this inaccessibility contributed to the plethora of space related culture that was created. This 1970s imaginary and material culture combined with inaccessibility closely resembles Lemmonier's account of 1950's car racing culture and suggests a similar dynamic of childhood experience becoming adult practice, that was enacted when I became a Kicksat supporter.

As a child the promise of a personal space craft or satellite seemed both feasible, and at the same time impossibly unobtainable, something reserved for Bond villains, corporations, and governments. The KickSat project proposed to make good on the promise.

\subsubsection{Cost}

The Kicksat project aimed to democratise access to space and drastically reduce its cost.

My goal is to bring down the huge cost of spaceflight, allowing anyone from a curious high school student or basement tinkerer to a professional scientist to explore what has until now been the exclusive realm of governments and large companies. By shrinking the spacecraft, we can fit more into a single launch slot and split the costs many ways. I want to make it easy enough and affordable enough for anyone to explore space. (Manchester, 2011)

\footnotetext{
7 “Cooperation In Space” (n.d.)

${ }^{8}$ O’Hare (2015)
} 
Physical access to outer space is extremely difficult to achieve and historically access to space has been both technically complex and expensive, with state space agencies until recently the only organisations capable of launching satellites into orbit. In this statement Manchester focuses on cost and affordability primarily, but also in highlighting 'the exclusive realm of governments and large companies' and wanting 'to make it easy enough' he also raises the issue of administrative and bureaucratic access as a second barrier to access. I will first look at the issue of cost as it relates to the TWOi project.

First I would like to consider how declining launch costs have impacted personal spacecraft development. Jones (n.d.) documents how over time the cost per kilo of launch to Low Earth Orbit dropped considerably from $\sim \$ 1,000,000 / \mathrm{kg}$ for early Vanguard systems, $\$ 61,700 / \mathrm{kg}$ for the Space Shuttle and $\$ 1,400 / \mathrm{kg}$ for the Falcon Heavy. NASA aims to reduce this cost to $\$ 200 /$ kilo by 2025 , and Space-X estimate that with fully reusable rockets the price may fall to as little as $\$ 20 /$ kilo.

The price of launch fell to a low level first with the Saturn 5 rocket and then plateaued during the Space Shuttle era, beginning to fall again with the commercialisation of the Russian space industry and more radically in 2010 with commercial launch vehicles such as Space-X commercial Falcon-9 and more recently Rocketlab’s Electron.

\begin{tabular}{lllrll}
\hline Year & Launch & Form Factor & $\mathrm{kg}$ & $\$($ USD) & $\$ / \mathrm{kg}$ \\
\hline 1958 & Vanguard & Satellite & 1 & $1,000,000$ & $1,000,000$ \\
1981 & Space Shuttle & Satellite & 1 & 61,700 & 61,700 \\
2010 & Falcon-9 & Satellite & 1 & 2,700 & 2,700 \\
2018 & Falcon-Heavy & Satellite & 1 & 1,400 & 1,400 \\
\hline 2013 & Roscosmos (OSSI) & Cubesat & 1 & $\sim 100,000$ & 100,000 \\
2019 & Electron & Cubesat & 1 & $\sim 50,000$ & 50,000 \\
2010 & Interorbital (Mur.at) & Tubesat (proposed) & 1 & $\sim 8,000$ & 8,000 \\
2010 & Celestis & Custom /femtosat & .007 & $\sim 6,000$ & 86,000 \\
2011 & Kicksat (Space-x) & Cubesat/femtosat & .005 & 300 & 90,000 \\
\hline
\end{tabular}

Table 3: Satellite Launch Costs

The falling cost of launching a satellite has very recently brought access to orbit into the realm of small organisations and even individuals. As discussed earlier the major cost of the the 2013 art satellite OSSI-1 by Hujon Song was the launch itself which cost \$100,000 USD for a $1 \mathrm{~kg}$ Cubesat payload. While still expensive for an individual this cost is within a range of personal affordability at approximately the level of a luxury car.

In November 2011, KickSat offered to radically lower cost further with an ultra small form factor 5gm satellite delivered and launched for \$300 (USD) or \$1000 (USD) for a developer version. 
Here we can see that the cost per kilogram of launch has drastically reduced since the early days of space exploration, but that satellite form factor and size is also extremely important for the overall project cost. Though Falcon Heavy in common has a very low cost per kilo, the spacecraft it is designed to launch are very large - up to 63.8 tonnes and it achieves cost savings largely by scale. For a personal satellite launch it is the total cost that is the limiting factor, rather than the cost per kilo and so an alternative strategy for reducing total project launch cost is miniaturisation of the satellite.

For KickSat the cost per kilo is actually higher than for the 1981 space shuttle launches, and the project cost reduction is caused by radically lowering the spacecraft mass to a few grams.

\subsubsection{Form Factor}

There is an ongoing trend in satellite design for cheaper, smaller and lighter satellites that reduces the cost of launch by reducing the mass of the satellite. As very small rockets are not economically feasible, small satellites have been enabled by ride sharing technologies where a single rocket launch can carry a number of smaller satellites. Ride sharing itself is enabled by standard form factors, and interface designs that create standardisation.

Over the last years the Cubesat form factor has emerged as the de-facto standard for nano satellites in the $1 \mathrm{~kg}$ - 10kg range, and has become the standard for small research satellites. The Cubesat specifies 10x10x10 cm cubes that can be stacked to make satellites of different unit sizes with $1 U, 3 U$ and $6 U$ sizes common. Each $1 U$ has a maximum mass of $1.33 \mathrm{~kg}$ and there are detailed specifications for what they may and may not contain Initiative (2017).

They are deployed from standard dimension Cubesat dispensers made by a variety of companies, such as Poly Picosat Orbital Deployer (PPOD), Nanoracks and Rocketlab's Maxwell. The dispensers can be attached as secondary payloads to rockets that have spare capacity, or combined into dedicated ride share launches. Multiple dedicated Cubesat launches are becoming common and in 2017 the Indian Space Research Organisation's (ISRO) Polar Satellite Launch Vehicle (PSLV) set a record for simultaneous launches with 104 satellites on a single rocket (Mathewson \& 2017, 2017). .

There is a parallel here between the Cubesat and the shipping container with its standard dimensions of 1 and 2 Twenty Foot Equivalent (TEU). Broeze (2002) describes in detail how the homogenisation of cargo into standardised units was a key development that enabled 
the globalisation of trade ${ }^{9}$. Containerisation provided a method of ride-sharing for cargoes, a standard form factor to improve handling, and created a simplified comoditised shipping market for goods. Further Broeze describes how though initially the cost of container shipping was higher, it created a clear physical, contractual and financial separation between the cargo owner, the shipping company, dock workers and their unions.

The Cubesat form factor standardisation similarly addresses the second barrier to access which is bureaucratic and administrative access. The standardisation not only creates a clearly defined form factor, but also a standardised contractual arrangement between satellite owner and launch operator.

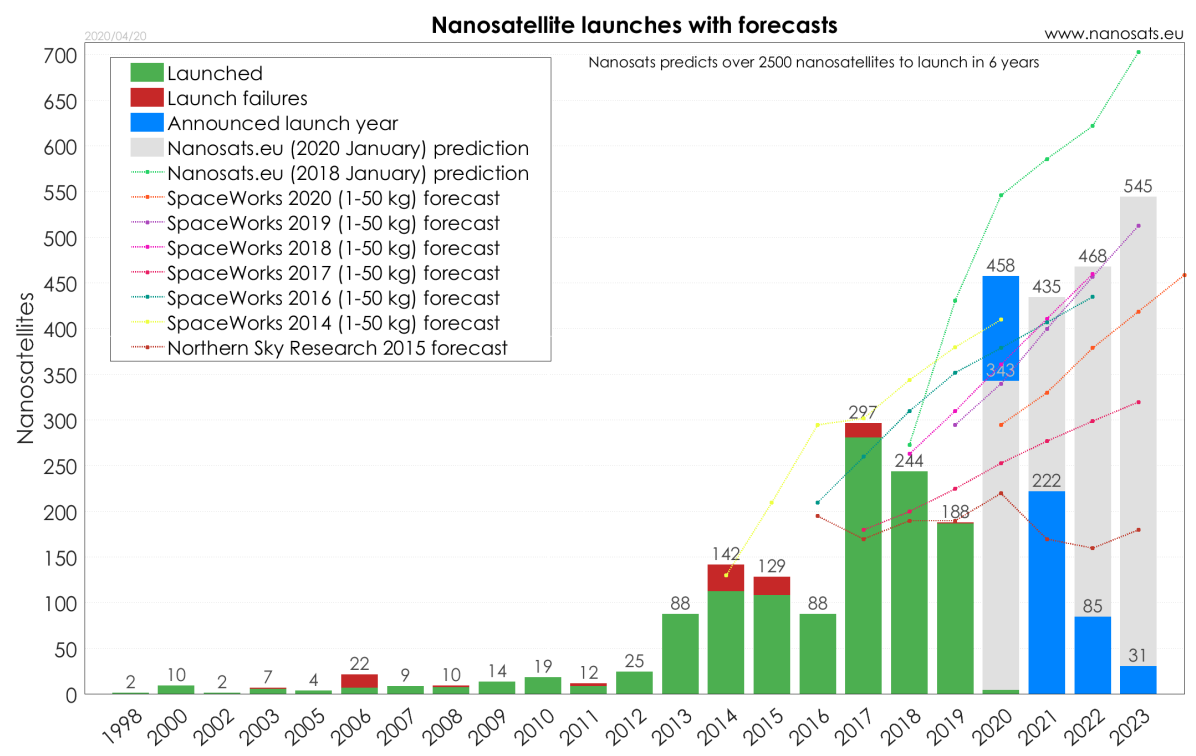

Figure 6: Nanosat Forecast

Note. Forecast of Nanosat usage.By Kulu (2020). With permission of Erik Kulu.

As the Cubesat form factor has emerged as a de-facto standard, so a commoditised market for Cubesat launches has developed with multiple vendors offering Cubesat launches to different orbits in much the same way as shipping companies offer passage to different ports. To date there have been 1317 nano satellites launched with 1210 of them Cubesats ${ }^{10}$ and 2500 are forecast for the next 6 years. The competing Tubesat form factor, has by contrast had 2 successful deployments. The popularity of the form factor is creating a market for off the shelf parts ${ }^{11}$ and launch bookings ${ }^{12}$ and this growth in Cubesat ecosystem, developers

\footnotetext{
${ }^{9}$ In 2002 containers carried $70 \%$ of global trade by value.

${ }^{10}$ Kulu (2020)

${ }^{11}$ CubeSatShop.com (n.d.)

${ }^{12}$ Isilaunch.com (n.d.)
} 
and deployers pushes back into entrenching the standard.

\subsubsection{Interfaces}

The clean physical separation between Cubesat and launch vehicle is an example of an interface. This is mirrored by the contractual separation of the contract which can also be considered an administrative interface. The modularisation of spacecraft is to a large extent controlled by interface specifications, and here it is worth looking more closely at concept.

Bratton (2015) uses an expanded definition of interface in his generalisation of the layer model beyond networks to the wider world. "An interface is any point of contact between two complex systems that governs the conditions of exchange between those systems." (Bratton, 2015) He emphasises crucially that interfaces both link or de-link users. In this diagram of the Cubesat interface both the technical and administrative interfaces are simplified, the Cubesat to a box, and the acceptance form to a 1 page checklist. 


\section{$1 U$ CubeSat Acceptance Checklist}

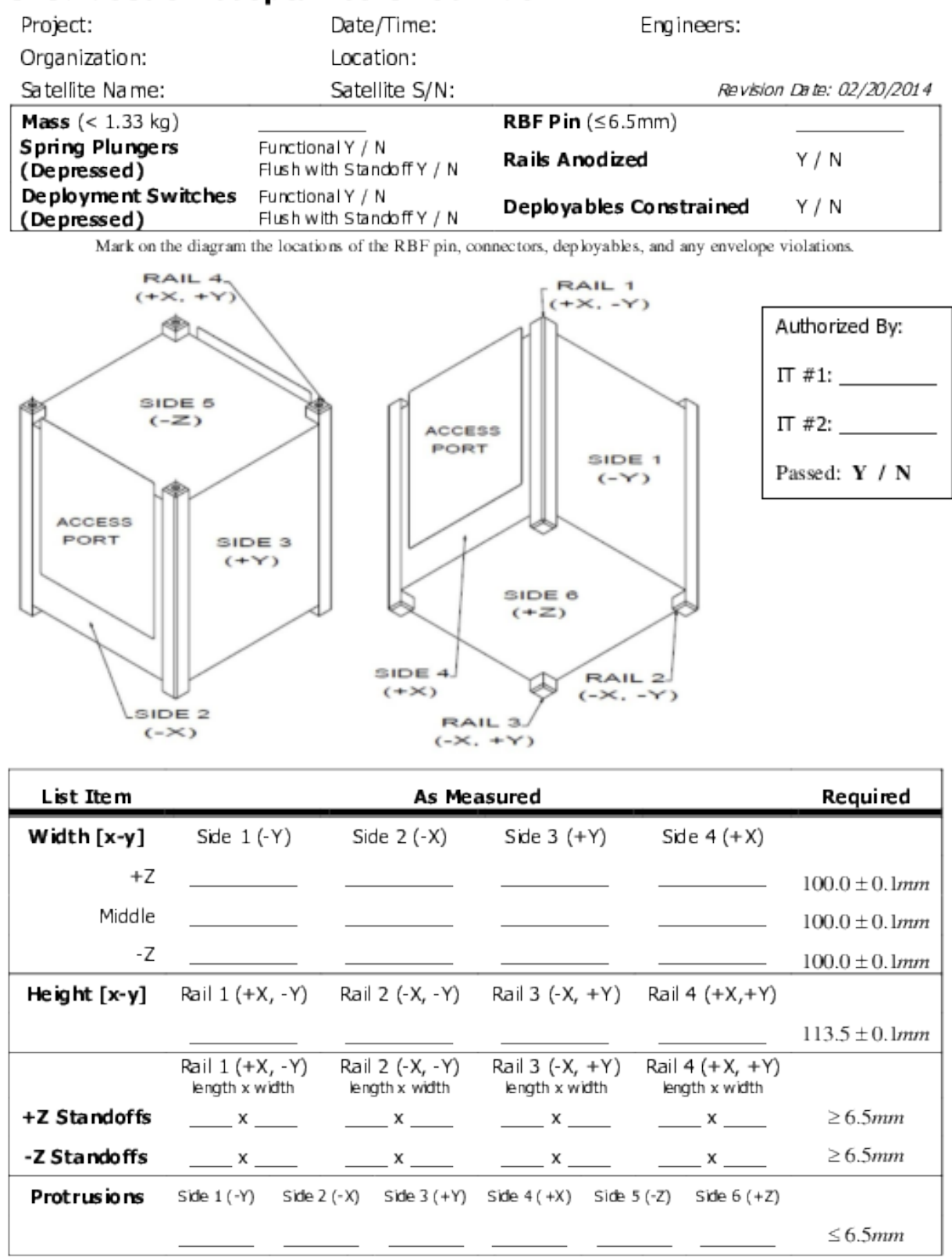

Figure 7: Cubesat Acceptance Form

Note. 1U CubeSat Acceptance Checklist. By Initiative (2017). Public Domain

Bratton foregrounds the user and describes the enabling and disabling character of interfaces for them, showing how interfaces define both what users can and cannot to do with a particular system. The interface defines not only the limit of the underlying system, but also the limit of the user and in this sense the interface can be said to define the user.

In discussing graphical user interfaces philosopher Wendy Hui Kyong Chun (2011) supports 
Bratton and proposes that interfaces produce users. "Interfaces and operating systems produce users — one and all." (Chun, 2011, p.67)

As an example she uses time sharing on Unix systems, where the user login and command line environment create the illusion of a personal space on a shared system. This artificial personal space then creates the limits of person hood for that system.

This process is similar to Leigh Star's concept of convergence in which, once an information infrastructure is established it begins to push back and structure the social world that produced it through use.

Convergence, again, is the double process by which information artifacts and social worlds are fitted to each other and come together (Star, Bowker, and Neumann, in press). On the one hand, a given information artifact (a classification system, a database, an interface, and so forth) is partially constitutive of some social world. (Bowker \& Star, 1999)

While here Bowker and Star refer to the convergence of an infrastructure and a social group, they go on to use the process to describe the evolving intersections between a personal biography and a classification system in an analysis of how Tuberculous diagnosis impacted a patients life. Without the classification of the disease, there was no forced hospitalisation. For them the personal is at least partially structured by the information infrastructure the individual is interfacing with.

Both early art satellites, ArtSat and Hujon Song's OSSI-1 used the Cubesat form factor, and Song's satellite set out to answer the question "Is a personal satellite possible?". After spending \$100,000 USD on launch Song answered "Yes, but it is expensive!" (Song, 2014a).

Here while the public narrative is again defined in terms of cost, the enabling factor is the standardised Cubesat form-factor. It is the Cubesat/launcher interface that creates the personal satellite and the individual is replicated in the Cubesat interface specification.

Here it is worth restating that the launch platform is shared with other satellites, and the Cubesat launch is not in this sense personal, but part of a larger project. There are other satellites, as well as a huge network of other individuals, organisations and systems involved in the launch of a ride share Cubesat, from launch operators, to regulators and tracking stations.

Even in the apparently personal case of Musk's Red Tesla launch or Beck's Humanity Star where the company CEO sends spacecraft as personal cultural/commercial statements, the 
launch is part of a complex network of relationships and the 'personal' aspect is because of privileged position within those networks. By contrast in Song's case he describes himself as an ordinary private individual with no particular advantage, connections or resources. The personal satellite is an expression of personal choice and private autonomy but Song is also an artist and the personal here evokes long disrupted traditional ideas of artistic authorship. In all cases though the personal contrasts with the wider organisational context.

Here the interface neatly de-links all of those other actors in a predictable and standard way, as well as creating a contractual arrangement that can be paid for by an individual. The Cubesat interface here produces an illusion of 'personal' users on a shared platform in the same way as Chun's Unix logins. This illusion is given legal status by the contractual arrangements between legal individuals, persons and companies.

\subsubsection{Community}

In this section I look at how community engagement in the KickSat project was conceived and operated. KickSat was named after popular crowd funding platform Kickstarter which emphasised the community aspect of the project. The Kickstarter campaign generated widespread media profile for the project and also created a potential community of practice ${ }^{13}$ for KickSat through the pledge campaign backers themselves.

\begin{tabular}{lr}
\hline Reward & Backers \\
\hline Name & 67 \\
Name + replica & 67 \\
Initials transmission & 88 \\
Developer Kit & 26 \\
Launch Button & 1 \\
\hline Total & 249 \\
\hline
\end{tabular}

Table 4: KickSat Backers

The first 2 groups pledged a minimum amount and received souvenir sprite satellites or initials etched on a plaque on the satellite. The third group of 88 committed to owning a sprite in orbit which would transmit a short message back to Earth, typically a person's initials or ham radio call sign. The last much smaller group of 27 purchased a developer kit and were planning to write and fly their own code on an individual sprite. At launch there were only 9 developer who flew their own code. ${ }^{14}$

\footnotetext{
13 "A community of practice is a group of people joined by conventions, language, practices, and technologies (Lave and Wenger 1992)."(A. P. Bishop, Van House, \& Buttenfield, 2003)

${ }^{14}$ Each Sprite satellite had a pair of PRN numbers which were unique identifiers that could be used for decoding
} 
For the different backers the form that the 'personal' took was quite different. The majority of backers were able to personalising the KickSat deployer satellite with their initials or broadcast a personalised short transmission from space. For the much smaller developer group the ability to write and fly custom code on a sprite constituted a much more personal level of control or ownership of the sprite spacecraft. This difference was reflected in the interactions of the two groups with what can be described as the user interface of the KickSat project. The personalisation group were able to send a few characters of text by email and had the potential to receive transmissions from space, but no ability to program the satellite. The developer group by contrast had full low level access to the processor and the sprite's systems via programming, and therefore the autonomy to create their own missions. The control was not total as the hardware design was set, and there were classes of code that handled radio transmission that were required to be installed, but for developers the personal access was perhaps equivalent to a collocated server. Ownership of the satellite was equivalent to the sense in which a user with root access to a server is said to $O W N$ it, and the financial contribution to the project constituted something like a contractual obligation. These two factors of contractual obligation and functional control commonly constitute personal ownership. In Chuns's terms these interfaces actually constitute the user/programmer/owner of a personal spacecraft. The different qualities of the developer and personalisation group's interfaces creating different levels of autonomy for those created users.

In contrast to Song's explicitly personal satellite, the development of personal spacecraft was not however the original intent of KickSat. The lead KickSat developer Zachery Manchester was based at Space Systems Design Studio (SSDS) at Cornell University where KickSat was his $\mathrm{PhD}$ project and he and a group of other students were responsible for developing the project. The core group were also holding a range of upstream relationships in the project, applying for licenses from various agencies, negotiating a free launch with NASA, positioning the work as a research project withing the academic framework at Cornell, and publishing on the work.

In a 2011 paper Manchester and Peck make a case for the chip sized femto satellite and developed the dynamics of chipsat sprites as well as showing the early Sprite prototype depicted in the Kickstarter campaign. In this paper the motivation for the small scale de-

transmissions from the satellite. When the final PRN radio identifiers were published $<2014-03-14$ Fri $>$ there were 9 developers in the list who actually had developed code to fly on the sprites with 124 transmitting only initials or call signs. 
sign had purely scientific goals. Space exploration and research is inherently uncertain, and having a large number of spacecraft working in unison can provide redundancy and hence higher success rates, allowing for novel forms of mission planning.

Chipsats are a promising new technology for space exploration, offering not only a new size scale for spacecraft, but also a new way of thinking about their design and mission planning. The potential to use vast numbers of them for sensing could open up new opportunities in fields like atmospheric sensing and planetary science. Manchester and Peck (2011)

Nowhere in the paper is the idea of a personal spacecraft, or individual ownership explicitly mentioned.

\subsubsection{Development Process}

From my point of view as a developer and Kicksat pledger there was a long wait of 13 months between the close of the campaign in Dec 2011 and the delivery of the developer kits in Jan 2013. During this period Manchester and the team at SSDS did the majority of the development work on the Sprites. The project was fully open source and during this period Manchester sent regular updates through the Kickstarter update mailing list with 33 posts about the ongoing development. He posted various hardware design and software commits through a GitHub repository that had it's first commit March 1, 2012.

Author: Zac Manchester <zrm3@cornell.edu> Date: Thu Mar 1 16:39:26 2012 0500

first commit

(https://github.com/zacmanchester/kicksat/)

In open source software development, developers contribute their individual code to a common code base under licenses which ensure sharing. They are then able to use and amend code from that common code base, receiving a benefit from the group's work that outweighs their individual contribution. Weber (2004) emphasises the personal motivation in this process and frames it in terms of an autonomous individual action. "At the center of the open source process are autonomous individuals who make choices about what they do with their limited time and energy." (Weber, 2004) 
The technical co-ordination of code submission between multiple individual users is typically achieved using online software repositories of which GitHub is one of the most common. A commit is the process of updating the repository with a user's new version. By looking at the commit history of a software project one can see who has been responsible for which bits of the development.

Despite the creation of a small group of developers through the Kickstarter campaign, all code development on the Sprite and deployer in 2012 was done by Manchester at SDSS and at this stage the open source code base largely enabled outward distribution rather than active participation.

In January 2013 the developer kits arrived and Sprite developers could then start work on their own code projects. The Kicksat Sprite was designed to be familiar to a wide range of hobbyists. It used an MSP430 chip set from Texas Instruments and Manchester had compiled a version of Energia for this chip set that presented an integrated development environment (IDE) based on the popular Arduino microprocessor IDE. Manchester provided sample code through GitHub and arranged the basic radio transmitting functions in libraries that could easily be loaded in the code. The programming language was $\mathrm{C}, \mathrm{C}++$ in common with Arduino and Manchester presented the sprite developers with a well rounded development environment that was familiar to any experienced Arduino developer and the majority of programmable electronics and physical computing makers and hobbyists. Libraries with the underlying radio transmission functions were provided in classes for inclusion in the standard way for an Arduino project.

Normally in larger open source projects there is a large quantity of online documentation, forum discussions, stack overflow articles and a wide community of developers working in similar environments. This was not the case for Kicksat and there were only 9 active development teams, with only 2 posting anything about their work publicly, notably the British Interplanetary Society (BIS) and HackSatOne teams. From my email record I can see that when I did have questions I addressed them directly to Manchester rather than to a community. The TWOi code was completed in 2013 and sent to Manchester who compiled it and installed it onto a Sprite that was integrated into the Kicksat deployer Cubesat ready for delivery to NASA/Space-X for launch. 


\subsubsection{Person}

As Kicksat backer and developer and 'owner' I was the person described in the phrase 'your personal satellite', with roles that changed throughout the project development. Initially the desire for a space project was a childhood dream and I was in a role of outsider or observer. The first step towards an active project was the meeting with an individual with a personal connection to the space industry and this lead to notification of the KickSat project. The Kicksat project resonated with the dream and I applied, on-boarding the Kicksat project. It was an open access project, was crowdfunded and open sourced and this allowed me to become a participant with a simple financial transaction. On making the transaction I entered into a community of practice and become actively engaged in space research. To start with I was an interested party accessing a one way flow of information from the Kicksat project. With the arrival of the physical device and developer kit, I changed role again and became a Kicksat developer, able to create software to fly on my 'personal spacecraft'. In my case this was as far as the relationship extended; I did not become someone who submits patches to the Kicksat project, have them accepted and contribute more directly to the project or become a core developer.

Note. Personal recollection.

"The Interface layer consists of any technical-informational machine, compressed into graphical or objective formats, that links or de-links Users and the Addressed entities up and down columns within the Stack." (Bratton, 2015)

To use Bratton's terminology the key interfaces that the user/developer connects with in this sketch are the two online platforms Kickstarter and GitHub, and the Arduino-like software development environment. These three interfaces perform a similar role to the standardised form factor of the Cubesat we looked at earlier. They both link and de-link the developer from the Kicksat project, and the stack of projects behind it that ultimately provide 'personal' access to space.

Here though it is clear that these platforms perform quite different functions for different users and that they are more fluid than straightforward interfaces. Susan Leigh Star (2016) describes these types of situations and develops the concept of the boundary object that operates across social situations. 
"Boundary objects are objects that are plastic enough to adapt to local needs and constraints of the several parties employing them, yet robust enough to maintain a common identity across sites." (Star et al., 2016, p.253)

Further Leigh Star outlines a taxonomy of boundary object types, three of which, terrain with coincident boundaries, ideal type, repository, map well onto the different platforms outlined above.

In this taxonomy the Kickstarter platform is perhaps a terrain with co-incident boundaries in which participants share the common boundaries of the platform, but address radically different contents and outcomes depending on their role. The platform is a way of accessing space exploration for the pledger, but a way of funding and publicising an existing space exploration project for the developer.

For Leigh Star an ideal type is a boundary object which is adaptable to a local site because it is fairly vague, understandable by all parties in that it can serve as a useful map for collaborative action, but also indeterminate enough that it can be interpreted in quite different ways. The idea of the personal spacecraft operates in this way here, providing a vague road map or goal, but having personal and heterogeneous meanings. The childhood dream of a personal spacecraft interacts strongly with the declared vision statement of the Kicksat project as a 'personal spacecraft' and this in turn provides a vehicle for the scientific exploration of the small satellite with its focus on mission redundancy.

"The source-code repository itself becomes a kind of boundary object, and functions as a meeting and merging point." (Ciesielska \& Petersen, 2013)

In contrast, the GitHub repository provides Leigh's repository boundary object in which the information is the same and shared, rather than vague. Here the source code in the repository is actually the same but the use of the code is heterogeneous. For the Kicksat project the code repository is the end point of publication, whereas for the Kicksat developer it is the starting point of development. The software repository as boundary object is explored in more detail by Ciesieslka and Pedersen (2013) who examine in detail how the repository can serve as a neutral point for resolving trust issues between diverse social groups involved in a project, for instance providing a meeting point and framework for dispute resolution in collaborations between hacker communities and commercial corporations with very different agendas. Here there was no dispute but the Kicksat project the goal was to test the radio technology of the Sprite, while the developers were producing their own projects using the 
Sprite as platform.

Internally within the code the pattern of object oriented software development code is used, and the interfaces themselves are presented as $\mathrm{C}++$ libraries or classes. These are encapsulated code modules with clearly defined interfaces which can be accessed from the main code flow. Fuller and Goffey (n.d.) frame this arrangement as division of labour that gives shape to a technicoeconomic hierarchy.

Indeed, a more finely grained division of the work of software development is made possible when the system or application to be built can be divided into discrete 'chunks'. Each class or class library (from which objects are derived) may be produced by a different programmer or group of programmers with the details of the operations of the classes safely ignored by other teams working on the project. (Fuller \& Goffey, n.d.)

While they focus on the power relationship of hiding data behind interfaces with encapsulation, in the context of an open source project where the code is visible, this acts as a soft power relationship that is nonetheless powerful. In the Kicksat project the encapsulation of code is a key infrastructure for producing an organisation from the disparate and otherwise disconnected group of Kicksat developers. Encapsulation is a technique by which a technicoeconomic hierarchy is produced that institutionalises the participants into a structure that can interact with the larger organisations responsible for launch and access to orbit.

But the type of institution that is formed around these boundary objects is both fluid, heterogeneous and hybrid. The mix of types of actor, hobbyist, graduate student, national space administration, commercial launch company, that are connected around the boundary object is very diverse, and yet functional. In terms of contemporary software development this type of arrangement is becoming common place, and O'Neil, Muselli, Raissi, and Zacchiroli (2020) document similar types of hybridisation in open source software development in some detail.

\subsubsection{Summary}

In this situation then, we have examined development process of the TWOi-project and how it interacted with and was enabled by the Kicksat project. Here we look primarily at the concept of a personal spacecraft and how it has operated through the project. First we have 
examined the Kicksat project on-boarding process and how the idea of the personal satellite resonated with the material and media culture of the 1970s and how this led to adult action using a model proposed by Lemonnier. Unpacking the stated and implicit goals of the Kicksat project, the historical reduction in cost of satellite launches is not the primary factor in improving access to space for individuals. Rather it is miniaturisation, standardisation, and form factor that play a key role and this is compared to containerisation.

Here I have outlined the features of the Cubesat form factor also with respect to the concept of the personal satellite. The large institutional and organisational framework which are necessary for spacecraft launch are shown to negate the autonomy of the individual and hence the idea of the autonomous personal spacecraft. The Cubesat form factor is de-linked from the launch organisation using Bratton's idea of interface and user. This interface is then theorized as creating the personal using Chun's production of the user by the interface. This is related to Bowker and Star's concept of convergence in which an infrastructure, in this case a form factor, both produces and is produced by interaction with personal biography.

The Kicksat project improves accessibility and achieves two orders of magnitude reduction in cost of space access over the Cubesat. It is proposed that this is actually achieved by a similar process to the form factor setting and interface definition of the Cubesat. Rather than a physical interface, the interfacing sites that enable this process are found to be the online platforms used for managing the development, of Kickstarter and GitHub, as well as standardised software environment and the underlying code encapsulation.

These platforms are discussed in terms of boundary objects and are found to map well onto Leigh Stars taxonomy with both repositories, terrains with co-incident boundaries represented. The relationship of the individual to the organisation is flagged with respect to open source software development which is framed as a hybrid organisation.

In addition the concept of the personal spacecraft is able to be described itself as a boundary object which provides linkage between the childhood dream and the scientific goals of the Kicksat project, that is itself productive of the community of practice.

In this situation the personal spacecraft as a discrete object launched into orbit by the autonomous will of an individual has been thoroughly critiqued. Further it is shown that an era of personal spacecraft is not driven by the cost reduction of launch related to the New Space conditions of commercial access. Rather the cost reduction is driven by the social conditions of access. 
I have outlined these condition as, the standardisation of form factors, interface specifications and open access to collaborative platforms. Taken together these create a range of boundary objects that enable the creation of communities of practice. These communities of practice are themselves open to individuals and the detailed structure of these boundaries creates a surface that constitutes a form of person hood. In short the 'personal spacecraft' is enabled not by a commercial cost imperative, but by a series of overlapping social structures. In all of this, the individual dream of space flight outlined at the beginning of the situation appears to be lost in the heavy social structuring of the environment. The sense of personal agency in this thicket of relationships is also diluted. And yet the sharp edges of these framing structures do create a space for personal agency that can be used to create artworks. How this sense of agency is recovered is discussed in the next situation. 


\subsection{Situation 2}

\subsubsection{Introduction}

The Kicksat project provided the satellite platform and launch capability for TWOi-project, and also set the timeline for the project. As with any project which involves Space or launch into orbit, the timelines were long and changed often for a wide variety of reasons. In the case of TWOi the project would extend eventually to almost a decade due to a sequence of events both foreseen and unexpected. In this section I examine in detail the evolution of the project during the 2014 launch and orbital period, and show how it was shaped by a variety of events. This narrative is then discussed with relation to concepts of protention and retention, contingency, emergence and agency.

\subsubsection{Planning}

At the time of the Kicksat campaign the project did not have a guaranteed launch date or even a guaranteed launch. The investment from the Kickstarter campaign was earmarked only for the build of the Kicksat deployer satellite and the sprite chipsats, not for the launch of the satellites. NASA's ELaNa educational launch programme started in March 2011 and offered applicants, primarily from university departments in the US, a free Cubesat launch into Low Earth Orbit. This represented a value of \$50,000-\$100,000 USD and was a way for NASA to stimulate interest in the industry, provide economic assistance and hands on experience to students wishing to enter the satellite industry. KickSat's plan from the outset was to apply to ELaNa programme for a free launch and this was stated in the campaign. On December 2, 2012 Manchester announced that Kicksat had been awarded a launch through ELaNA but with no firm launch date. With the launch secured, the Kicksat project was able to move ahead and the Kicksat team then started focusing solely on completing the development of the space craft, a process that would take the rest of the year.

Just a day after this I wrote to a friend Stella Brennan, a digital artist who was working on an exhibition celebrating the Transit of Venus at the Adam Gallery ${ }^{15}$. This is the first record I have of a description of The Weight of Information artwork using the satellite as material.

One result of our conversations about orbits was that I ended up buying that $\$ 500$ [NZD] KickSat $2 \mathrm{~cm} \times 2 \mathrm{~cm}$ satellite launch and just heard that NASA have

\footnotetext{
${ }^{15}$ Dark Sky | Adam Art Gallery (n.d.)
} 
confirmed a launch probably in 2013! I'm going to do a whimsical microgravity experiment to try and measure the effect of gravity on information called 'The Weight of Information'. Priest (2012)

The launch was confirmed to take place on the ELaNa-5 which was a Cubesat ride share launch on board SpaceX's CRS3 mission along with 5 other Cubesats. SpaceX-CRS3 was a commercial International Space Station re-supply mission whose contract had been awarded to SpaceX.

In 2006 NASA's Commercial Orbital Transportation Services (COTS) programme began to develop commercial resupply options for the International Space Station (ISS). After the 2003 Space Shuttle Colombia disaster and as the Space Shuttle programme came towards its end, NASA was looking for new ways to launch cargoes into orbit and to resupply the ISS. There was a geopolitical aspect in that during the early 2010's while the space shuttle programme was beset by difficulties NASA had to rely on Russian launch vehicles for resupplying the Space Station. Indeed NASA did not launch an astronaut from US soil between 2011 and May 30, 2020 when Space-X successfully delivered two astronauts to the ISS. A COTS contract was awarded to Space-X in 2008 who started development of the Falcon 9 rocket for cargo first and then astronauts. Space-X CRS-1 flew on October 8, 2012, with SpaceX CRS-2 flying on March 1, 2013.

It is worth noting here that the KickSat campaign had started a full year before the first Commercial Resupply Service (CRS) launch, and had received its launch award only early in 2012. On September 8, 2012 KickSat was manifested on Space-X CRS-3 with a target date of July 2013. This set a timetable for potential development of the artwork, but with the developers kits months away there could be no work on the TWOi satellite itself. (Manchester, 2011, September 12, 2012, Update \#20)

\begin{tabular}{lll}
\hline Announcement Date & Description & Launch Date \\
\hline$<2012-02-12$ Sun $>$ & KickSat ELaNa announcement & n/a \\
$<2012-09-08$ Sat $>$ & KickSat manifest on CRS-3 & no earlier than $<2013-06>$ \\
$<2012-11-29$ Thu $>$ & KickSat update on CRS-3 & no earlier than $<2013-09-30$ Mon $>$ \\
$<2013-05-04$ Sat $>$ & CRS-3 public date & $<2013-11-11$ Mon $>$ \\
$<2013-06-03$ Mon $>$ & CRS-3 date slippage & no earlier than $<2013-12-09$ Mon $>$ \\
$<2013-09-07$ Sat $>$ & CRS-3 delay & no earlier than $<2014-01-15$ Wed $>$ \\
$<2013-12-11$ Wed $>$ & KickSat integration & no earlier than $<2014-02-22$ Sat $>$ \\
$<2014-02-26$ Wed $>$ & Cape Canaveral Schedule & $<2014-03-16$ Sun 04:41:00 $>$ \\
$<2014-03-14$ Fri $>$ & SpaceX date change & $<2014-03-30$ Sun $>-2014-04-02$ Wed $>$ \\
$<2014-03-18$ Tue $>$ & Primary and backup confirmation & $<2014-03-30$ Sun 10:50:00 $><2014-04-03$ Thu 09:40:00 $>$ \\
$<2013-03-28$ Thu $>$ & Scrubbed: Fire at tracking station & no new date \\
$<2014-04-05$ Sat $>$ & reschedule & $<2014-04-14$ Mon $>$ \\
$<2014-04-13$ Sun $>$ & schedule confirmed & $<2014-04-14$ Mon 04:59:00 $>$ \\
$<2015-04-14$ Tue $>$ & Scrubbed: Helium leak on rocket & $<2014-04-18$ Fri $>$ \\
$<2014-04-17$ Thu $>$ & Schedule confirm: Bad weather & $<2014-04-18$ Fri 15:25:00 $>$ \\
$<2014-04-19$ Sat $>$ & LAUNCH: CRS-3 successful launch & $<2014-04-18$ Fri 15:25:00 $>$ \\
\hline
\end{tabular}


Table 5: KickSat Launch Evolution

After the initial launch announcement, the launch date was repeatedly revised so that the final launch date of Kicksat was April 4, 2019 around 9 months after the initial delay. These delays were all upstream from the Kicksat project mainly stemming from SpaceX issues with CRS-3. The TWOi code was delivered to Manchester by email as a small zip file on October 30, 2012 and was successfully installed onto a fresh sprite satellite, loaded into the KickSat deployer. The KickSat deployer was integrated into the CRS-3 second stage vehicle on December 11, 2013. As the launch approached the launch dates were again put back. The Falcon 9 rocket was even on the launch pad at one point but then the launch was scrubbed because of a fire at a radar ground station.

The process of launching a satellite starts from the moment it is booked on a launch vehicle and added to the launch manifest. The launch is given a provisional date which maybe a general period of the year and is typically specified as 'not before' a specific date. This date is then amended further into the future as the preparations of the spacecraft proceed. A cargo such as a satellite is given a delivery date for integration onto the launch vehicle and all development must be completed in advance of that date. As the launch approaches the launch date is revised to take account of the progress of the various sub-projects, and eventually a launch date is decided and a launch window defined. The launch window ${ }^{16}$ is a short period in which it is possible to insert the payload into a specific orbit. This gives an opportunity for short term interruptions such as weather conditions to be avoided. Typically a backup date and associated launch window is also defined. For Low Earth Orbit satellites there are many potential opportunities for launch - satellites which do not have to align with a specific object such as another spacecraft or the night/day terminator may be launched at any time. International Space Station cargoes must be inserted into orbits that rendezvous with the space station very precisely and these occur regularly every few days limited by the beta angle cutout (angle of the orbital plane to the Sun).

\subsubsection{Dependency}

The number of dependencies in the launch of a satellite is very large. The rocket itself is a highly complex technology with a huge number of parts, processes, designs, workflows and teams. In 2013 The Space-X launch vehicles were early in their operational histories, and

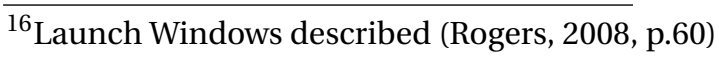


recently out of the design and testing phase, and the Falcon 9 rocket, while flight proven, could be considered to be still in a beta phase. In addition each primary payload has its own design and development processes and operational considerations. In this case the primary payload was a cargo delivery to the ISS. Not only did the payload create dependencies, but it included additional dependencies of all the experiments and equipment on board. The delivery endpoint of the ISS introduced yet more dependencies, and largely drove the schedule. Furthermore the launch took place in the context of a high stakes political and geopolitical process of developing a viable private US launch capability to provide NASA with a US based means resupplying the ISS.

Over the course of space history $5-10 \%$ of launches have failed ${ }^{17}$, the economic cost of failure is very high and even potential risks can cause a launch hold or scrub (abort). In rocket engineering and mission control there are many mitigation and planning strategies for eliminating potential failure modes. These attempt to limit contingency and create reliability and predictability for the launch.

Rogers (2008) describes the Mission Operation Plan that is put in place in planning a mission, and different organisations have clearly defined interfaces ${ }^{18}$, areas of responsibility, reporting obligations and checks.

In project management Ishikawa (1990) ${ }^{19}$ set out a series of tools for improving quality in projects, one of which is the cause and effect or fish bone diagram. The tool is used in mission design to analyse critical components of any system by enumerating the cause and effect chains that can bring about failures. These contingencies can then be eliminated or mitigated.

These contingencies are however passed down to downstream projects in the form of delays and schedule changes. Here I will use a terminology from software design of dependencies and coupling. In software design the network of dependencies is called a dependency graph and is a tree of conditions that must be met for the sequence of events to proceed ${ }^{20}$. The dependant linkage between software modules is termed coupling, and a common design principle is to reduce coupling between modules (Yourdon \& Constantine, 1979). A loosely coupled module can fail without taking the entire system down and this is termed graceful

\footnotetext{
${ }^{17}$ Orbital Launch Summary by Year (n.d.)

${ }^{18}$ The Spaceflight Mission Planning guide shows examples of interface requirements for cubesats.

${ }^{19}$ First published in 1968.

${ }^{20}$ In Anthropology chains of dependency are a very similar concept to dependency graphs (Hodder, 2012, p.52).
} 
failure. An interface is an example of a strategy for reducing coupling, and tightly coupled modules are termed dependencies with a map of these dependencies is called a dependency graph.

Here I show a simplified large scale dependency graph of the various organisations involved in the CRS-3 launch. The main launch mission CRS-3, was commissioned by NASA to resupply the space station. NASA also commissioned the development of the Dragon cargo spacecraft which was the primary payload on board. A secondary ride share payload was added by NASA to use spare capacity on CRS-3. This consisted of 5 Cubesats which were granted free launches under NASA's Educational Launch of Nanosatellites (ELaNa) programme. One of these 5 was KickSat and the Kicksat deployer carried 104 sprite chip satellites of which TWOi was one. The TWOi project consisted of this chipsat with custom software loaded on it to provide functions for the TWOi artwork.

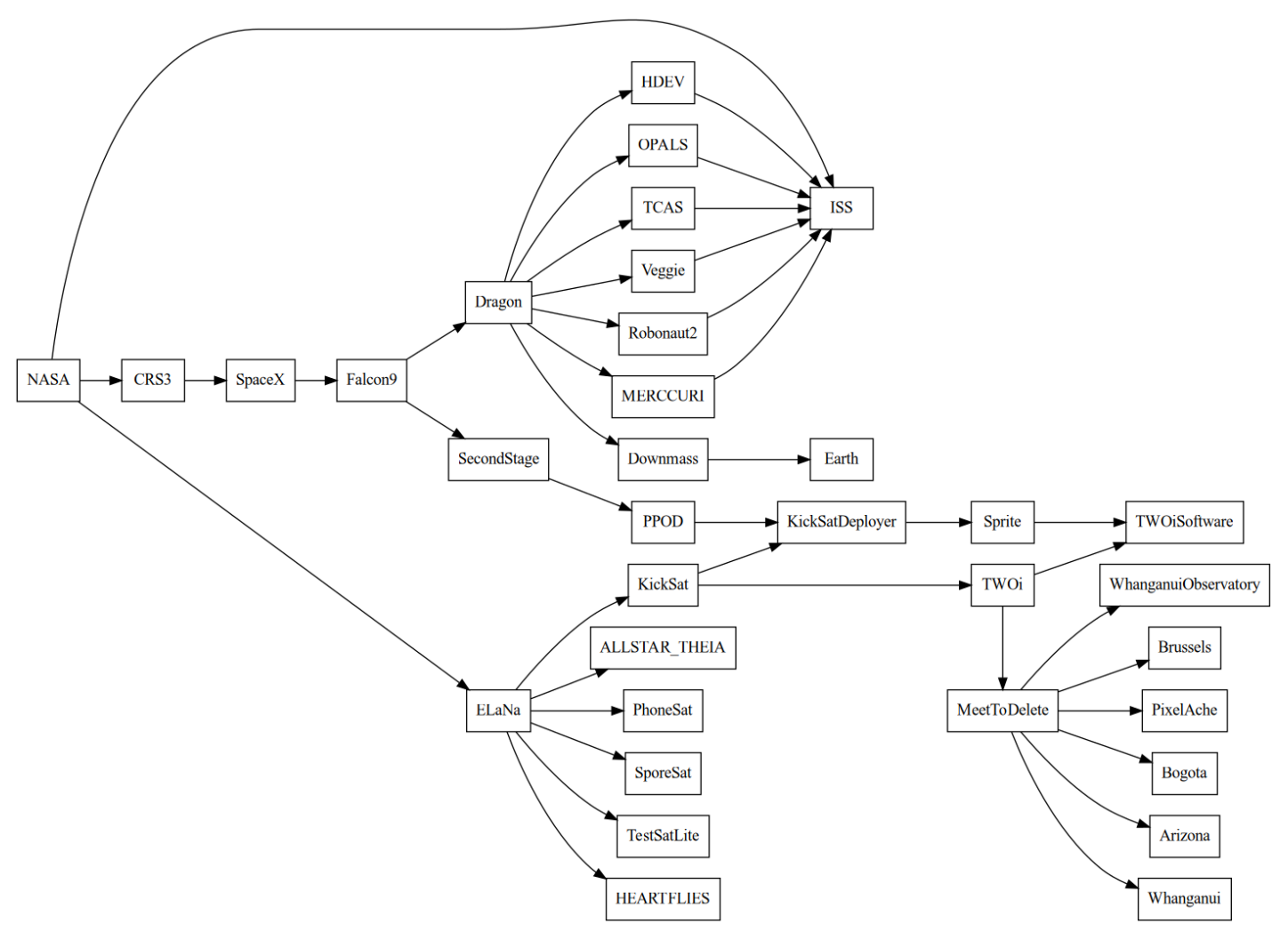

Figure 8: TWOi Dependencies

/Note./TWOi project dependency graph. Priest, J (2020).

Here we can see that the TWOi-project was positioned at the very bottom of the dependency graph and had effectively no influence on anything upstream. Even downstream notifications of status change were not always delivered and the TWOi project had to react quickly 
and change direction as situations developed. In short the TWOi-project was entirely contingent on the upstream launch mission.

The Kicksat project which is immediately upstream from TWOi-project likewise had no influence on the launch sequence and was unlikely to have been included in the Mission Operation Planning of the launch. Once the satellite had passed it's compliance checks and been integrated into the launch vehicle, it was effectively a passive passenger, though the project was directly privy to the messaging around launch schedules. As discussed in Situation 1, the Cubesat interface here provides a separation layer that simplifies not only the physical object of the Cubesat, but also it's interaction with the larger space craft, the de-linking in Bratton's terms. This de-linking attempts to remove the Cubesat payload as far as possible from the chain of cause and effect shown in the Ishikawa diagram.

The systematic removal of dependencies and reduction in coupling at a system and subsystem level by improving modularity and simplifying interfaces,is a key strategy in removing possible failure modes and thus contingencies for the launch, which must take place within extremely fine tolerances. Paradoxically this produces a form of contingency in the form of delays for the de-coupled sub project modules such as TWOi.

Hodder (2012) discusses this dual action in terms of networks of affordances, dependence and dependency, in which relationships are shaped by their materials, and he highlights that these are often unequal.

"Rather than a mutualism of affordances and dependence there is often a limiting and constraining dependency."(Hodder, 2012, p.51)

Here Hodder makes a distinction between dependence, which is productive and dependency which is limiting. In our case the rocket provides the affordance of launch into low earth orbit, and the dragon spacecraft, KickSat satellite and TWOi are locked in a productive dependence, that launches the satellite. This rocket launch evolution then becomes a dependency of limits and constraints for the TWOi project.

And since humans and things depend on other human beings and other things along long chains of dependencies, the dependencies are spread out across space and time. And as things are unruly and unstable, humans are forever chasing along the chains to fix things, forever drawn into further dependencies. Hodder (2012)

Rather than seeing these dependency chains as static, Hodder sees them as dynamic and 
responsive to unruly events in the world, that themselves create new dependencies, and this he terms entanglement. We see this process enacted in the pre-launch delay sequence that follows.

\subsubsection{Pre-launch}

A rocket launch can be cancelled or scrubbed at any time right up until a few seconds before the ignition of the main engines. In the case of Space-X Falcon 9 the last abort opportunity is at T minus 10 seconds. Prior to launch there is a pre-launch period typically of a few weeks before the launch in which the rocket is prepared. The countdown to launch starts between 1 day and 10 hours before launch. The launch attempt begins with the rocket being moved out to the launch pad and in the case of Falcon 9, countdown starts when the rocket is moved to the vertical position. The countdown continues with a predefined series of checks each of which can cause the launch to abort, and typically there are periods when the countdown can move to a $H O L D$ state. If the $H O L D$ state continues for too long the launch window will close and the launch will be scrubbed and re-scheduled.

In the case of the first KickSat launch the launch date was repeatedly changed and there were 3 separate launch attempts where the rocket was moved on to the launch pad in preparation for countdown. These delays had a major effect on the TWOi project and the development of the artwork. The TWOi artwork had developed over 2012 and the core design of the artwork was that it should be in two parts. The first part was the TWOi satellite in orbit running its information deletion routine as the satellite descended. The second part was a series of ground based events around the world that coincided with the satellites orbit. As discussed in Situation 4 this was a way of making the project visible to Earth based audiences and the events were conceived as participatory gatherings. At these gatherings participants were invited to 'Meet to Delete', to bring information of some sort to be deleted or destroyed while the satellite flew overhead doing the same. The intention was that telemetry data from the satellite would be transmitted to the events, along with position data from Kicksat and other feeds or telemetry.

\subsubsection{Meet to Delete}

These delays had a major effect on the TWOi project and the way the artwork developed. As described in section X, the TWOi-artwork consisted of two related parts, the first the satellite 
in orbit, and the second a series of ground based Meet to Delete events that coincided with the satellite's orbit. The events were participatory gatherings that were connected to the orbit by potential radio transmissions and other techniques that are explored in Situation 4 in more detail. Here it is worth noting that for public, the primary means of experiencing the artwork was through the participatory events. There were also secondary events associated with the orbital period such as a launch party, and media both online, radio and press. Beyond this the artwork was conceived in the first place as consisting of the participation between the authored situation of the events, the satellite and members of the public. It was intentionally designed to be open to and dependant on whatever occurred during the orbit. In discussing Deller's work The Battle of Orgreave, art historian Claire Bishop uses the phrase directed reality to describe this kind of authorship (C. Bishop, 2012; Deller, 2001).

This intentional directed reality principle for the work, means that the effects of the satellite and its supporting apparatus should be taken to constitute the artwork. Presenting the delays and contingencies outlined in this section, should not be taken as mere scheduling errors, but as constituent parts of the artwork.

\subsubsection{Launch}

The TWOi project and the accompanying Meet to Delete event series was presented at a number of symposia and conferences and an open call for participation was sent out to a variety of media art mailing lists and personal contacts, throughout 2013.

In 2013 TWOi was presented at the Ada Symposium in Dunedin, ISC4WN wireless networking event in Berlin, and the Media Art Histories Renew conference in Riga. From these events and others a group of curators, and event organisers responded and wished to participate in the project and to schedule events. Each event had an organiser, a venue, a publicity schedule and an audience or group of participants who needed to be updated on the project. With 11 events planned the dependency graph on TWOi expanded dramatically. At the time of organising the event series I was not aware of the likelihood and nature of launch delays to come. As the series of delays came through the event organisers had to be managed so that the events would coincide with TWOi's orbit.

In Whanganui, the home town of the TWOi project, the Whanganui observatory was offered by the Whanganui Astronomical Society as a suitable venue for watching the launch and inviting local participants to attend. The Whanganui Observatory hall was converted into a 
budget mission control with 2 projectors, live video and a telemetry feed from Space X and NASA.

The local publicity was managed tightly with a local newspaper and the launch event was only publicly announced the day before the launch. At the first launch attempt the event was held on a Friday evening. A large crowd gathered at the observatory, including members of the local media, and then the launch was scrubbed and instead a talk on the project was presented. At the second launch attempt the local time was in the morning, and a whole school class were invited to the launch and catering provided in the form of doughnuts - a NASA launch tradition apparently. The launch was again scrubbed and a talk on the project presented. The third attempt was very early in the morning around 5am and did not look likely due to the weather. The event was attended by only a few immediate family and friends and members of the Whanganui Astronomical society.

It was this launch that was successful and after a rapid ascent and short first stage burn, the Dragon spacecraft separated from the second stage and headed towards the ISS. As the second stage fell away the PPOD launchers were visible on the top of the rocket stage, and after a short time KickSat was deployed into its own orbit.

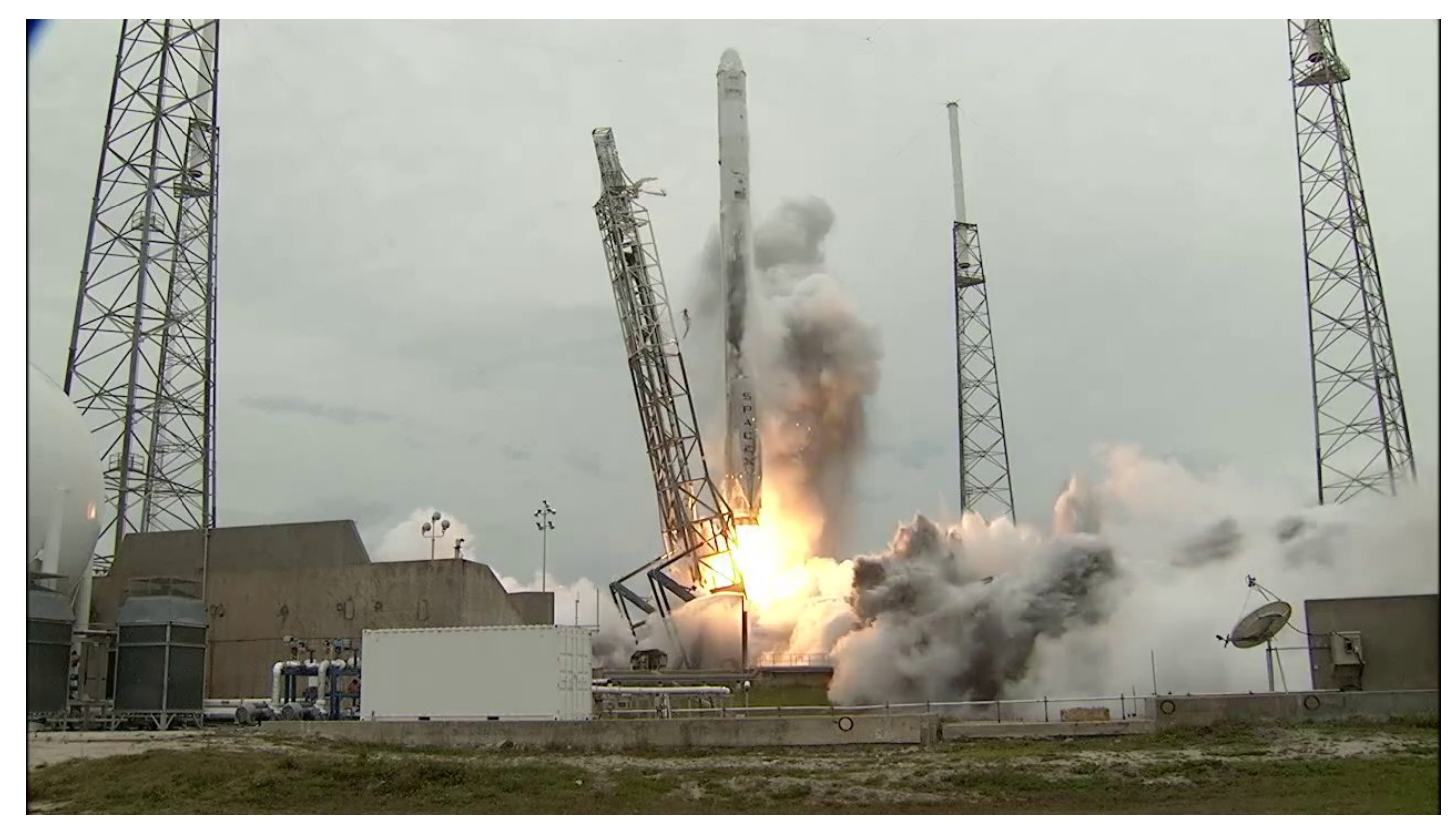

Figure 9: CRS-3 Launch 2014

Note. Screen grab of CRS-3 Launch video. By NASA's Kennedy Space Center (2014). Public Domain. 


\subsubsection{Orbit}

After the KickSat deployer was ejected from the PPOD dispenser it was placed into a 16 day separation orbit. The separation orbit was a security measure designed to place the KickSat deployer on the other side of the Earth away from the ISS to prevent any potential debris issues when the chipsat Sprites were released. The Sprites themselves would be placed on an intentionally rapidly decaying orbit with an expected duration of 1 week before re-entry and burn up of both KickSat deployer and Sprite constellation.

The KickSat deployment triggered the start of the TWOi artwork event series and the Meet to Delete events began to occur. At this point the TWOi satellite was not yet in autonomous orbit itself, but with an unknown spaceflight evolution - the KickSat deployment was as close to a firm starting date as possible.

The KickSat deployer booted and started sending telemetry data back to Earth which was published by the KickSat team. Manchester put out an open call for Radio Hams and other amateur satellite observers to attempt to observe the KickSat telemetry transmissions. Updates were published through a Google Group email list which became the main point of contact for both KickSat developers and Radio Amateurs following the project.

Following the KickSat deployment Manchester offered prizes for people observing and recording KickSat telemetry packets and these started being sent in to the mailing list. Manchester would decode them and publish the telemetry data and this became a significant feed on the mailing list. In this sense the openness of the project to participation utilized and existing international community of radio amateurs to provide an informal ground station network with a worldwide geographic spread at effectively zero cost to the project. In addition the open publication of the telemetry data created a strong sense of involvement from all the KickSat participants who could track the status of the satellite.

As the Cubesat group from the PPOD deployment spread out they were assigned different tracking numbers and Two Line Element (TLE) sets began to be published on Celestrak and other satellite tracking websites. A TLE is a short text file with a group of 14 fields arranged on two lines. A TLE defines any Earth orbital object's identification, precise location and direction of travel at any time. The TLE contains administrative information such as Satellite Catalog Number as well as inclination, right ascension and other positional data. Satellites in LEO are affected by drag from the thin upper atmosphere and so their orbits evolve over time. Tracking stations such as the North American Aerospace Defence Command (NORAD) keep 
track of the satellites and update and publish the TLE as they receive positional fixes from tracking stations. Ground stations on the Earth's surface use the TLE information to point their antennas. LEO satellites, in common with the ISS move at high speed and will cross the night sky at any given location in a short time. Kicksat, at $250 \mathrm{Km}$ above Earth made a pass over any given point a few times a day, passing from horizon to horizon in around 15 minutes or less. The TLE data, fed into a tracking software such as Gpredict, can produce azimuth and elevation data. This data can be used to automatically steer a motorized directional antenna across the sky, allowing it to point in the correct direction to detect the satellite's radio signals. Much of the technology for this is open source and while certainly a technical challenge, comes within the budget and skill set of quite a large group of radio hams and amateurs.

In the case of KickSat the radio hams were called on to help this amateur open source satellite project, and many took part providing a much greater range of international ground station coverage for the Kicksat project than would otherwise have been the case. An additional incentive was the technical challenge of decoding the transmissions from the sprites themselves. The radios on board the chipsats were very low power outputting $10 \mathrm{~mW}$ Effective Isotropic Radiated Power (EIRP) from 2 small wire antennas ${ }^{21}$. They used a novel radio transmission schema based on gold codes and software post processing to increase the signal to noise ratio and enable the signal to be heard on Earth. The gold code encoding is similar to that used in GPS where each bit is encoded as a long string of ones and zeros which represent a gold code. The string is like a massively redundant key with the same signature encoded repeatedly, so that if only a small fragment of the code can be recovered, it can be used to identify the transmission. Theoretically this encoding could produce a gain of $56 \mathrm{db}$ equivalent to a very large dish antenna and this gain could make a faint signal from the chipsat discernible on Earth.

Testing this novel low power transmission and encoding schema was the formal scientific goal of the KickSat project, and to some extent the rest of the project, including the crowd funding and all the individual sub-projects were enabled by this scientific research question. The receiving and decoding of the signal required software defined radio equipment to receive it, and high power computer processing to decode. The schema was not able to decode the signal in real time and recordings of transmissions were sent to Manchester for

\footnotetext{
${ }^{21}$ For comparison 10mW EIRP is approximately $1 / 3$ of the power output of a standard home WiFi router.
} 
post processing. It is possible that the novelty of the whole approach was also attractive to radio amateurs and a group became involved in tracking the KickSat satellite. A few Kicksat developers also created their own ground stations, while others equipped themselves with basic hand held equipment and a third group relied on updates from the project.

This mixed community congregated around the Google Group, and shortly after the initial deployment telemetry data was being collected, decoded and shared on the list, with Manchester giving out prizes in the form of mission patches for early sightings of the satellite and detailed telemetry from the onboard flight computer.

The separation orbit continued smoothly and a steady stream of telemetry packets were recorded, decoded and posted on the mailing list showing the status of the KickSat deployer. The mailing list discussion focused on new sightings, how to decode the Sprite transmissions after the release of the sprites and some decay predictions for when the satellites would reenter and people were using the telemetry beacons to practice following the KickSat for the upcoming deployment

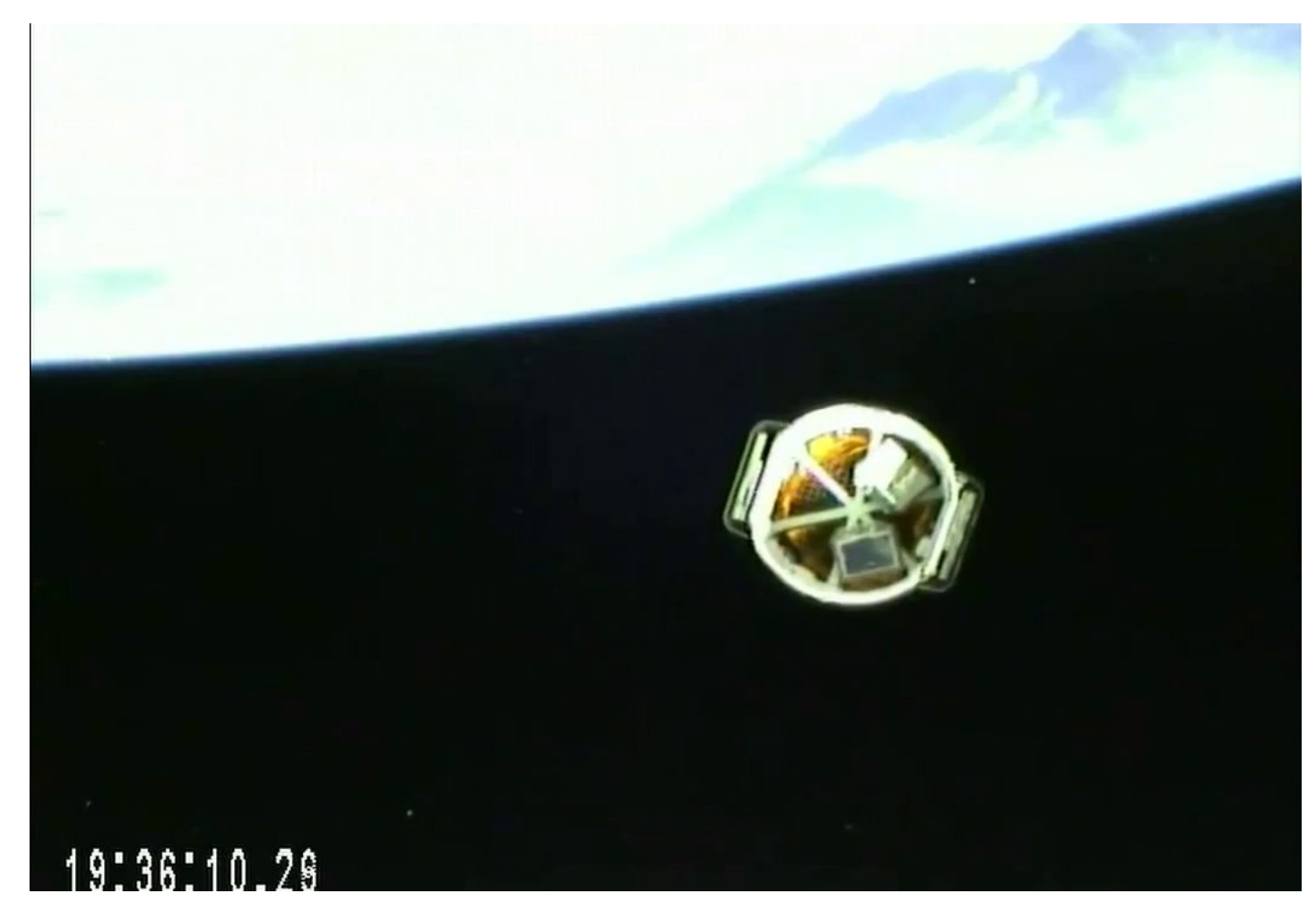

Figure 10: 2nd Stage Separation

Note. Frame grab from CRS-3 2nd Stage Separation Video. By Center (2014). Public Domain. 


\subsubsection{Reset}

On April 30, 2018 Ron,G8FJG posted this truncated packet from a pass in orbit 188.

"only received this?

1:Fm KD2BHC To CQ Via TELEM <UI F/R Pid=F0 Len=14> [18:18:48R] P4C,664,0,0,0,"

(https://groups.google.com/d/msg/kicksat-gs/OKOBXqzY7Rc/mI9ukSTVjBMJ)

[(KickSat-gs, 2012)]

The record from orbit 186 had been normal, but this short form packet showed that the main flight computer on the KickSat had shut down to preserve power and the backup flight controller chip was in control and sending out only a truncated status message. The Kicksat deployer satellite used 2 separate flight computers. The first was based on PhoneSat, another budget Cubesat development effort that used modified mobile phones to make low cost satellite flight computers. The second backup used a very simple embedded microcontroller called a Picaxe that ran a basic routine which Manchester characterised as the 'reptile brain' of the satellite'.

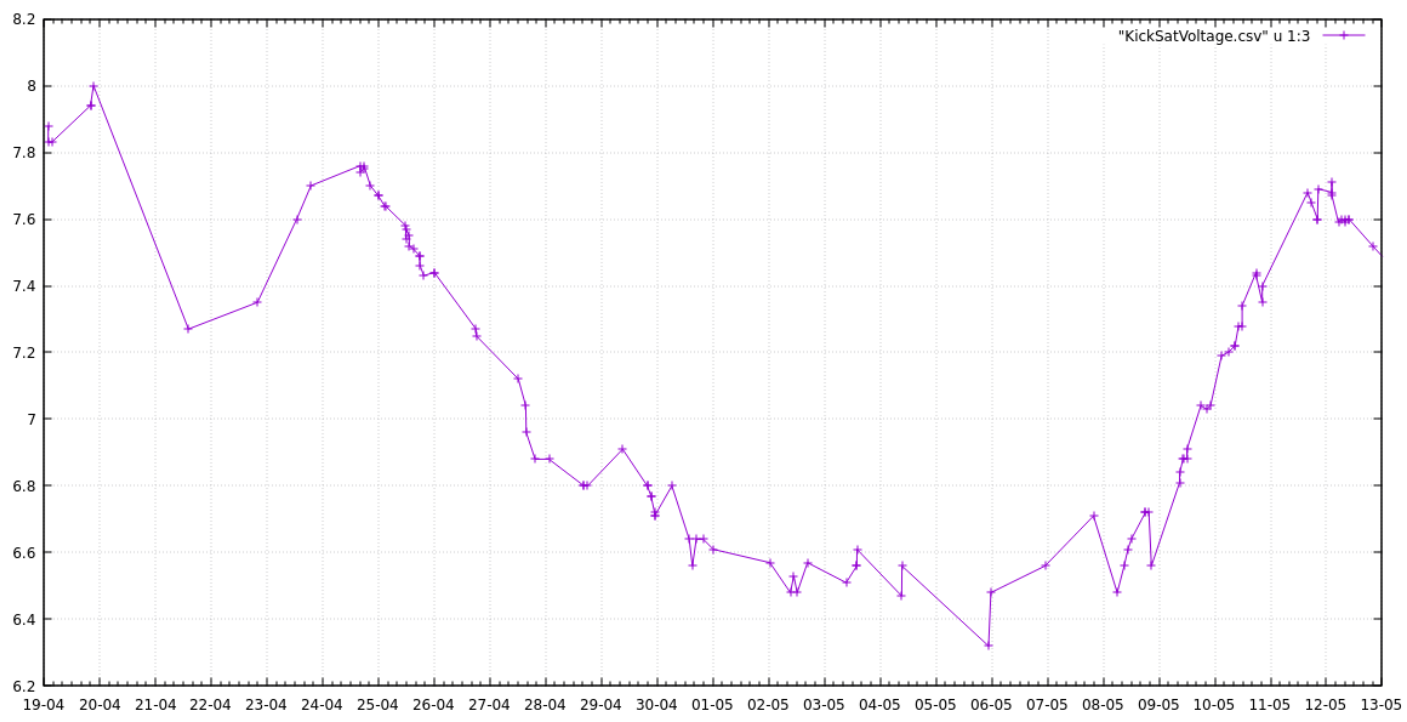

Figure 11: Kicksat Deployer Voltage

Note. Time evolution of Kicksat Deployer Satellite Voltage. Data is drawn from a shared public Kicksat decoded telemetry data from the KickSat-gs google group (Kicksat-gs, 2014). By Julian Priest, cc-by-sa.

Over the course of the flight the voltage of the batteries powering the PhoneSat had been steadily declining indicating that the solar panels were not providing enough power to recharge 
the batteries to keep the main flight computer running. The PhoneSat was responsible for releasing the sprites, and also was able to receive signals sent to it from Earth. The watchdog was unable to receive commands but was pre-programmed to release the sprites at the end of the 16 day separation orbit as a fail safe mechanism. Then on April the 30th the watchdog reset itself, and started counting days again from zero.

As most of you have noticed by now, the packets coming from KickSat have changed in the last couple of days - they have reverted back to the shorter packets that were transmitted when KickSat first launched. This was due to a hard reset of the "watchdog" micro-controller on KickSat - the sort of "reptile brain" of the satellite that manages turning on and off the rest of the subsystems and keeps the master clock. It appears the reset happened some time in the morning (EDT) of Wednesday, April 30th. The reset doesn't seem to be the result of power issues (the watchdog should run until the batteries reach $5.5 \mathrm{~V}$, and they've been holding steady around 6.5 V). Instead, it seems the likely culprit was radiation. (https://groups.google.com/d/msg/kicksat-gs/elwNkubIbLw/ Mc9bvITCE1QJ) [(KickSat-gs, 2012)]

\begin{tabular}{llll}
\hline Date & Solar Wind & proton flux & xray solar flares \\
\hline$<2014-04-29$ Tue $>$ & $306.1 \mathrm{~km} / \mathrm{s}$ & $2.0 \mathrm{p} / \mathrm{cm}^{3}$ & B9 2254 UT \\
$<2014-04-30$ Wed $>$ & $324.5 \mathrm{~km} / \mathrm{s}$ & $8.7 \mathrm{p} / \mathrm{cm}^{3}$ & $\mathrm{C} 10620 \mathrm{UT}$ \\
$<2014-05-01$ Thu $>$ & $368.7 \mathrm{~km} / \mathrm{s}$ & $3.4 \mathrm{p} / \mathrm{cm}^{3}$ & B70142 UT \\
\hline
\end{tabular}

Table 6: Space Weather April, 2014

/Note./From (Spaceweather.Com Time Machine, 2014, April 30, 2014).

Looking at the solar activity for the period of the reset there was indeed a sharp spike in high energy proton activity similar to what could be expected during a solar minimum and there was some speculation that Kicksat could have passed through the South Atlantic Anomaly (SAA), a known area of high cosmic ray activity so radiation is a possible explanation.

\subsubsection{De-orbit}

This was though a disastrous turning point for the project because KickSat had a predetermined and extremely short flight time. In order to minimise the chance of space debris, KickSat was on an aggressively downward spiral trajectory and was due to re-enter the atmosphere and burn up around May 14, 2014. With the flight computer out, and the backup 
counting down the 16 days of the separation orbit again, KickSat looked like it would re-enter before having a chance to release the sprite satellites.

It became clear that the only hope for releasing the satellite would be if the batteries recovered to $7 \mathrm{~V}$ at which point the PhoneSat would boot and would be able to receive a message to deploy the Sprite chipsats.

\section{"10/05/2014 Юрий Мироненко}

As the mission comes to end, more and more eyes tracking it:)" (https: //groups .google.com/d/msg/kicksat-gs/sK1cPxEnhX4/dt08IujFF4YJ) [(KickSat-gs, 2012)]

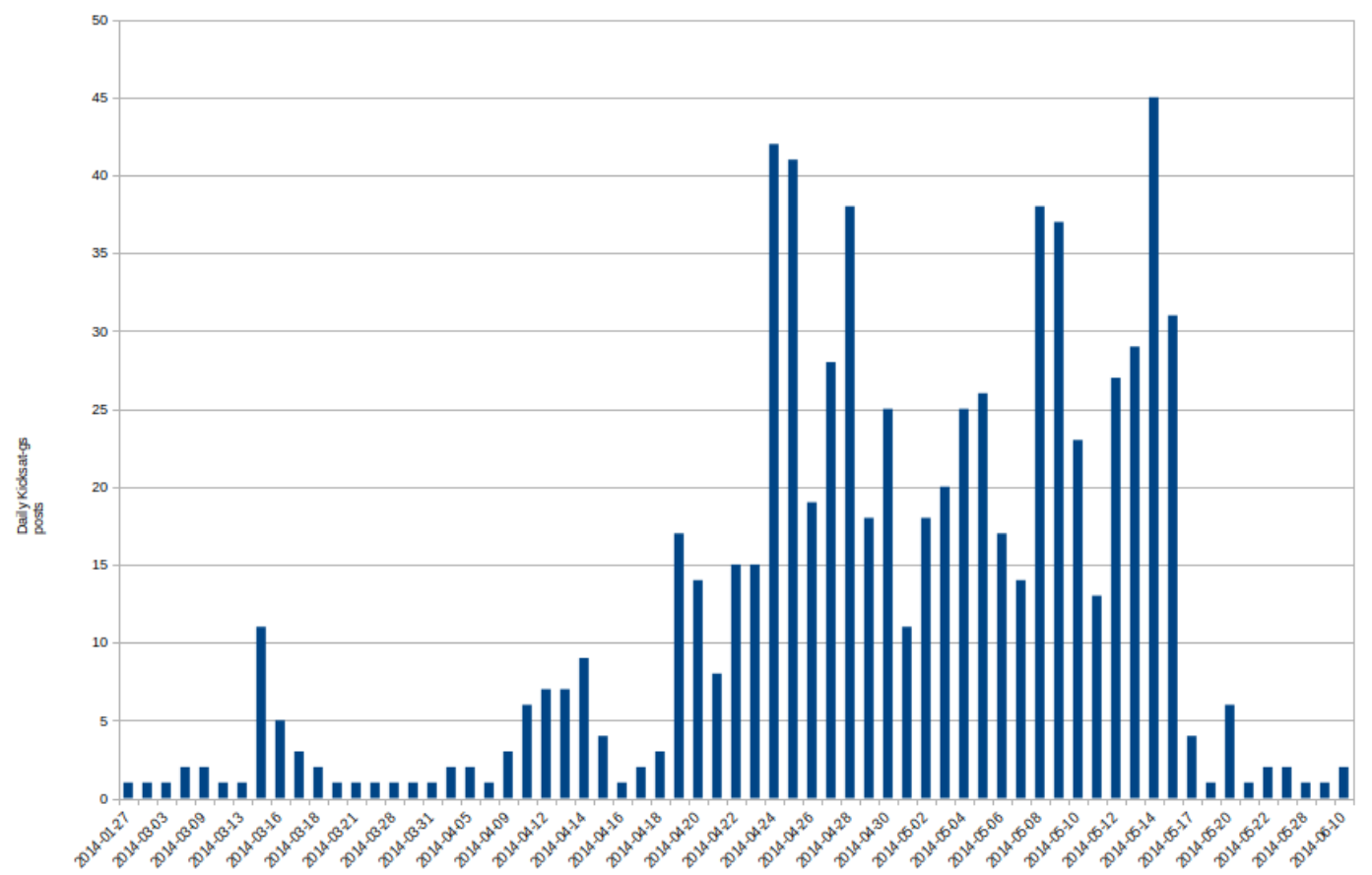

Figure 12: KickSat-gs Google Group Posts

/Note./KickSat-gs Google Group posting frequency by date. By Priest, J (2020).

The effect of the news of the potential demise of the project was an increase in interest in the project and extra activity on the mailing list. The effort to collect and collate the transmissions from the satellite increased and a heated discussion about the likely decay of the satellite orbit and potential re-entry date. The position of the satellite was known and constantly updated from the beacon, but the dynamics of re-entry from LEO are complex and accurate re-entry time and location prediction is inaccurate. The upper atmosphere varies in thickness at different locations and times of year, and the dynamics of low mass objects 
travelling at high velocity is challenging. There were lively discussions about varying models and assumptions and the KickSat decay prediction thread grew to 79 posts complete with calculations and graphs.

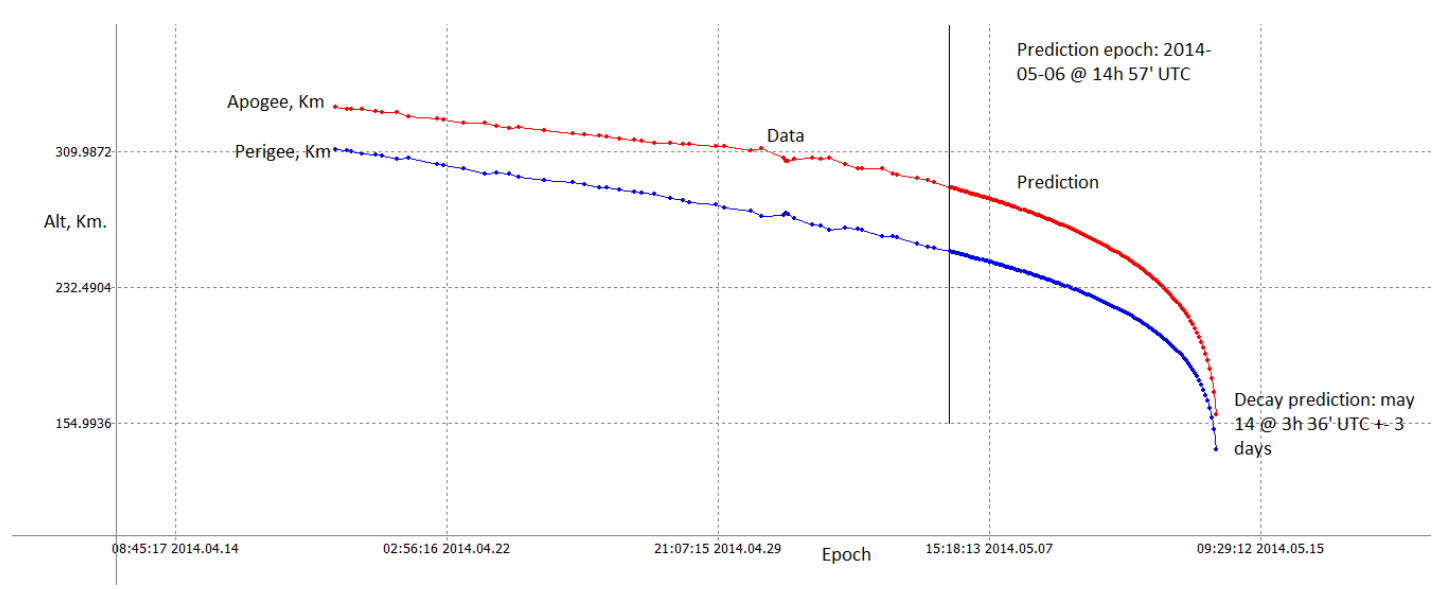

Figure 13: KickSat Decay Prediction Curve May 6, 2014

Note. KickSat Decay Prediction Curve posted to KickSat-gs. By Mikel (2014). With permission of Jon Mikel.

These three curves of slow voltage increase, orbital decay and increase in community involvement taken together create a picture of a moment in the narrative of the project. The potential loss of KickSat and it's cargo of 104 chipsat sprites occurs through a combination of technical failure and external intervention in the form of cosmic rays. The slowly increasing voltage curve represented hope that the situation could be saved by human intervention while the decay prediction curves represented the probably end of the KickSat's flight. Faced with this opposition, the community increased in activity to do anything that might help to save the situation and release the sprites and TWOi into autonomous flight.

Eventually the KickSat did not recover enough power to reboot the flight computer and on May 14, 2014 at 01:30 UTC during orbit \#404 KickSat re-entered the atmosphere somewhere over the Sahara desert, and burned up with all the chipsat sprites, including TWOi unreleased inside.

The Meet to Delete events went ahead as planned during the orbital period, but took on a poignancy as the doomed satellite fell back to Earth and was itself destroyed. These events were now contextualised by the classical tragedy unfolding in orbit. 


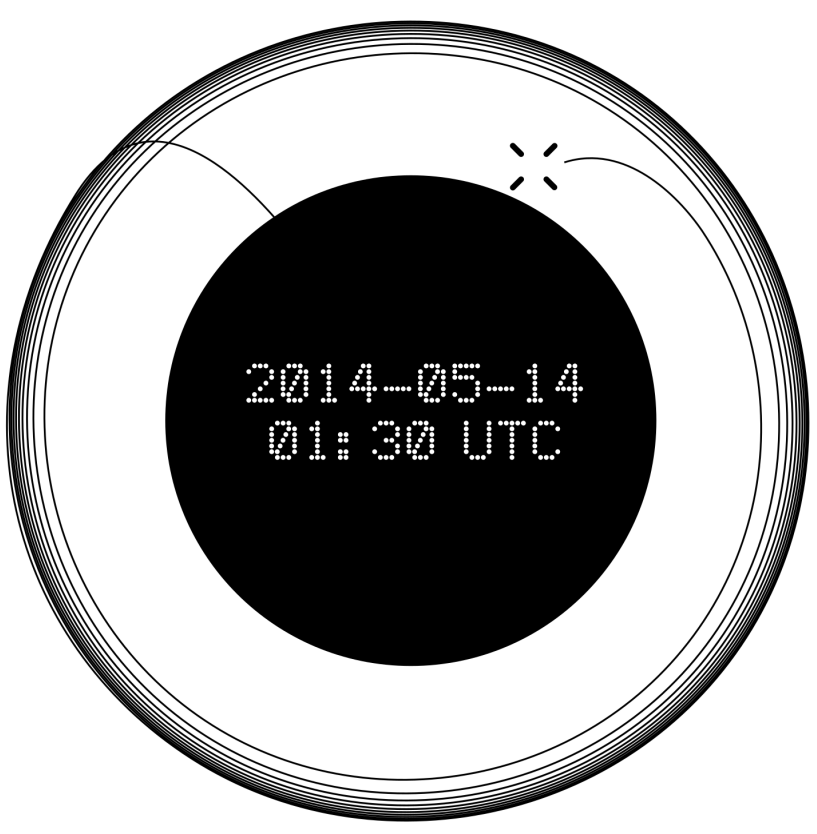

KickSat and TWOi re-entered Earth's atmosphere on May 14th 2014 01:30 UTC during orbit \#404.

Due to a timer reset caused by a cosmic radiation event on April 30th 2014 the TWOi sprite satellite was never deployed into autonomous orbit and is presumed to have burned up somewhere over North Africa inside the Kicksat deployer satellite on re-entry .

$\mathrm{EOF}$

http://julianpriest.org/twoi

Figure 14: TWOi Re-entry Poster 2015

Note. Poster and online graphic published on the occasion of TWOi re-entry (Priest, 201405-2014). 


\subsubsection{Contingency, Agency and Time}

The TWOi project is clearly framed and presented as an artwork, but one in which the primary physical object, the TWOi satellite is remote from the viewer and not directly available for perception. The potential outputs of the work are only visible in the highly technically mediated form of post processed radio transmissions, tracking station reports, telemetry data, derived positional graphics, and postings on various Internet platforms.

In Art and Agency, Gell (1998) rejects the idea of art as being concerned with symbolic communication and places the emphasis on agency, intention, causation, result and transformation. "I view art as a system of action, intended to change the world rather than encode symbolic propositions about it." (Gell, 1998)

TWOi fits this description well and repeatedly uses all of these elements in its development. There is intention both at the outset in the initial framing and throughout the project; intention to launch, intention to orbit, intention to delete information, intention to meet to delete. The entire process is highly contingent with only a slim possibility that the project as described at the outset will go ahead as planned. TWOi is authored and designed to some extent but the process is both framed by the surrounding organisational constructs and open to rapid change and evolution through a range of different processes, bureaucratic conditions, technological choices, social actions and many potential and actualised non-human and environmental potential interventions such as cosmic ray events.

Gell develops the case for artworks as distributed objects across time and space. Rather than describing an artwork as a singular object he sees an artwork as a corpus or loosely integrated set which can span periods of time and distance. In an analysis of Duchamp's Network of Stoppages (Gell 1998), he describes the work in terms of an overall framework of corpus or oeuvre even though it appears to be a singular work. Network of Stoppages was a precursor study for a part of the Large Glass, a work whose final form was also affected by the contingent fact of its breakage during shipping (Henderson, 2005).

Network or Stoppages was itself repeatedly reworked, incorporated other existing works, and was painted over. The work adapted through a series of recognisable individual works which are resting points on a journey to the final form. The work in turn depicts a network of stoppages and Gell describes the intermediate artworks through which the work passes as a network of perchings. "The sum total of the infinitely transformable network of internal references (protentions and retentions) uniting the work from all of these temporal 
'perches'..." (Gell, 1998, p.250)

To describe the processes within a corpus he uses the concepts of protention and retention drawn from Husserl's phenomenology of temporality Husserl (1929). Husserl is concerned to create a theory of the flow of time in which moments are not discrete but can have influence on the experience of the past or influence the expectations of the future.

At any given moment as events pass from present into past they sink into progressively fading memory. As events are anticipated in the future they arise into perception from nothing until they firmly take shape. Not only this but in a continuous flow of time any past event colours the experience of the present moment; the past experience is retained in the present and informs the expectation of the future, and this is the process of retention. Similarly an expectation of a future event and its likely consequences informs a present moment in a process of protention. Further protention and retention differentiate from straight anticipation and memory in that the protention of a future event can colour the agency of a past event, and vice versa.

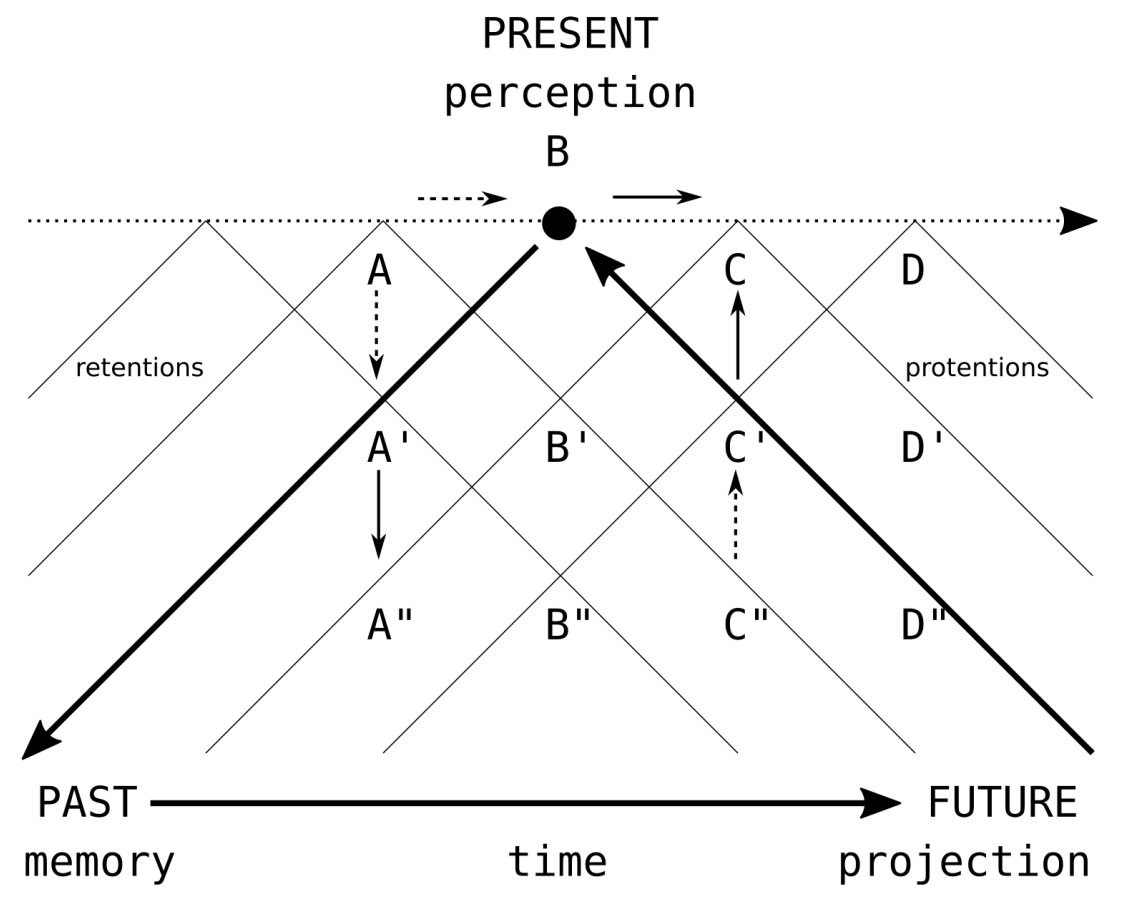


Figure 15: Gell's Protention and Retention

Note. Simplified version of Gell's protention and retention diagram showing the effect of past events on future events and vice versa. Gell's diagram was derived from Husserl's original. By Julian Priest.

Considered as an artwork TWOi is temporally extended over a long period of time and changes constantly throughout the development. In this sense TWOi can be considered as a time based or durational work, albeit one of indeterminate duration, and the processes of protention and retention can be modelled.

To take the example of the launch announcement described above the launch was initially announced and this created an anticipation of the launch taking place on a certain date. As the launch date approached the protention of the launch structured activity in the TWOi project and curators for meet to delete events were identified and events scheduled. At this early stage there was no expectation that the launch would not happen. When the launch date was put back protention of the future launch date again structured the present moment in re-scheduling but this time retention from the previous launch attempt reduced the certainty of the future event. In fact the retention of the previous event reduced the protention of the future event thus destabilising the present. Over the course of repeated launch delays and failures the expectation of a successful future launch receded until by the actual launch the protention was so weak that efforts to rally a 'public' for the launch event were much reduced and the launch at The Whanganui Observatory was watched by a handful of people. In another example of this process the period from actual launch to re-entry was marked by successive changes in circumstance. After the successful deployment of KickSat attention was focused on the forthcoming sprite release. This idea of the future release structured behaviour and the mailing list discussion concerned mainly plans for the ground station and telemetry reports. The retention of the successful launch and deployment created a protention of a successful sprite deployment and a relaxed tone on the mailing list traffic.

After the catastrophic failure of the PhoneSat and the counter reset the stark possibility of a future re-entry without sprite release and the burn up of the KickSat created a negative image of the immediate future and a strong protention added to by a strong retention of the recent past failure of the flight computer. The possibility that the project would have a negative outcome structured activity on the mailing list, and there was both a strong effort to understand the flight dynamics as well as some recrimination and disappointment from 
some members of the community.

The rising voltage chart represents the hopes of the PhoneSat rebooting, a message being relayed, and the sprites being released in a successful outcome. The falling decay prediction chart represents the fears of re-entry occurring before sprite release and a disastrous outcome for the project. At a midpoint between the flight failure and the re-entry the future evolution of the project is uncertain. A pair of outcomes each create protention into the present, and the two recent events of successful launch and computer failure create retention. This results in something akin to dramatic tension that stimulates discussion, and real world action from the community. The actions of training antennas and analysing telemetry data are undertaken on the voltage/hope side, and manipulating beacon location reports, upper atmosphere models and decay prediction software on the decay/despair side. This tension creates discussion, disagreement and at some times conflict on the mailing list.

This period of uncertainty was also responsible for an increase in interest in the project, and something like a coming together of the various disparate groups of the community. The developers, radio hams, Kicksat backers and art event participants are all co-opted into a classic tragic narrative Eagleton (2002), and one in which all the participants and stakeholders are invested; the Kicksat developers with their scientific research programme, NASA with their large in kind donation, the radio hams with their time and attention, the gallery participants with their venues, schedules and time, and of course the artist with reputation etc.

Tragedy is the imitation of an action, not of human beings. By and large, it is events which are tragic, not people.... One might claim rather that for Aristotle any sort of shift of fortune will do which evokes in us pity and fear. (Eagleton, 2002, pp.77-78)

Eagleton argues that classical tragedy is concerned with events that befall the protagonist. In the TWOi-project the satellite as protagonist, looses its agency and its fate is settled by contingent events, evoking audience pity and fear in the audience.

In Husserl this model of temporal flow is proposed to get around the problem of a Newtonian description of time in which points on an absolute time line are largely independent and sequential. Husserl's protention/retention model spreads the effect of time over a sliding window using anticipation and memory as mechanisms to effect action in the present relative to views of the past and future. It should be noted here that Husserl is concerned 
only with phenomena, or events as experienced and so this is not a physical theory as such. The image has more than a passing resemblance to Minkowski's light cones which vividly describe what can in principle be visible to an observer under special relativity, and there is a deeper connection here between the physical and experiential descriptions of time. The smooth nature of phenomenological time-consciousness was first expressed in lectures by Husserl in 1905 (and later published by Heidegger in 1929), and 1905 is the very year when Einstein published 'On The Electrodynamics of Moving Bodies' in which he first developed special relativity.

Alves (2008) makes a compelling case for the strong connection between these descriptions of physical time and experiential time and goes so far as to attempt a synthesis establishing a philosophical grounding of physical time in experiential time. Whether one goes as far as this or not, the concept of a smooth continuous time embedded in flow where the relative situation of the observer/participant is foremost in structuring either the local physical or experiential and hence social dynamics of a moment.

\subsubsection{Discontinuity and Entanglement}

In the evolution of TWOi this smooth manifold of experiential protention and retention is however disrupted. Rather than a smooth transition from one state to another we have short duration events which cause disruption to the smooth unfolding of situations. A fire at a radar installation on the US East Coast destroys tracking capability and causes the rocket launch to be scrubbed as does the subsequent helium leak on the rocket. The impact of a cosmic ray, likely a single proton causes the backup timer to reset and changes the entire direction, and outcome of the satellite flight.

These events are highly discontinuous, unpredictable and contingent, and Rorty here uses a cosmic ray as an example of novelty in a mechanistic universe of blind, contingent, mechanical forces.

For genuine novelty can, after all, occur in a world of blind, contingent, mechanical forces. Think of novelty as the sort of thing which happens when, for example, a cosmic ray scrambles the atoms in a DNA molecule, thus sending things off in the direction of the orchids or the anthropoids. (Rorty, Rorty, \& Richard, 1989, p.17) 
The helium leak is unlikely, but perhaps in principle knowable with enough prior knowledge, or analysable after the fact as with the O-ring diagnosis of the Challenger disaster $(\mathrm{Au}-$ risicchio, Bracewell, \& Hooey, 2016).

However a single proton hitting a memory register and resetting a micro-controller is not even in principle knowable being a quantum event. It is entirely possible, (though not in principle knowable) that an in-principle uncertain event could cause the disruption of an experience of future and past time.

Here I am rhetorically mixing, experiential and physical time, but nevertheless the discontinuities and rapid shift from one state - anticipation of successful deployment, to another anticipation of re-entry are mediated by very small events and subtle interplay of situation. The image of the future and the past rapidly shifts dependent on situation and the train of external events.

Rather than a smooth manifold of time this points to fractures, fragments, discontinuities or some kind of discreteness in our experience of time. "What happens when machines stop working? Could this form of work stoppage be considered a form of agency?". (Barad, 2007) Barad (2007) directly addresses discontinuity and the disruption of the mechanistic and deterministic by the quantum in her theory of agential realism. This is based on close reading of Bohr's epistemology of apparatus in which the apparatus must be attended to as an integral part of the phenomena being investigated. From this she derives a concept of intra-action which is grounded in quantum entanglement and she describes it as "the mutual constitution of entangled agencies". In this philosophy time and space are not Newtonian containers in which events occur, but are "intra-actively produced in the ongoing differential articulation of the world". (Barad, 2007, p.33)

The existence of the quantum discontinuity means that the past is never left behind, never [U+FB01] nished once and for all, and the future is not what will come to be in an unfolding of the present moment; rather the past and the future are enfolded participants in matter's iterative becoming (Barad, 2007, p.234).

This has consequences for agency, which looses it's alignment with human intentionality and allows for matter to become agentive. For Barad then the enactment of the cosmic ray proton resetting the microprocessor is itself the agency, and this changes the range of possibilities for the next iteration of becoming. This range of possibilities allows for an open future, foreclosing strict determinism and limiting complete free will. 
Barad inverts at a fundamental and physical level Husserl's protention and retention acting inside a continuous flow of time, instead time is produced from the enactment of intraaction with a similar effect to Husserl's protention and retention.

Whilst Hodder (2012) develops his concept of entanglement to resolve problems of affordance, dependency and contingency with respect to macroscopic objects and material culture, Barad arrives at entanglement as the core of agency from a microscopic quantum perspective.

\subsubsection{Summary}

In this situation then we have seen the way in which the TWOi-project and the evolution of the TWOi-artwork were heavily affected by the evolution of the KickSat project from planning through to de-orbit.

This has been described in terms of contingency, coupling, dependency and finally entanglement. The command and control structures of the rocket launch, whilst attempting to eliminate contingencies, create new ones in the dependent projects. The evolution of the situation in time was then viewed through the lens of protention and retention, and the changing expectations and experiences shown to influence the community reaction to events. These key points can be viewed as a network of perchings from which the TWOi-artwork emerged. This emergence was enabled by TWOi's explicit framing as an open participatory artwork, that involves participants in the evolving situation.

The key events themselves point to a highly discontinuous time manifold, and in this light Barad's agential realism and Hider's entanglement provide excellent tools for describing the situation. While coming from radically different disciplines and scales of quantum physics and archaeology, these two theories provide a way of understanding the way in which a single microscopic event can structure a network of entanglements that are focused on the event. Crucially here entanglement recovers a form of agency from what appears to be an entirely constrained environment, and this is centered on the material object of the satellite.

Even with the recovery of agency, this situation poses significant practical problems for design or artistic authorship, that is implicit in making work of any kind in the outer space environment, and especially cultural work. The technical environment that allows access to space or rockets is necessarily highly structured and within extremely fine tolerances, and this creates a highly entangled situation for any payload. Cultural projects at the bottom 
of the chain of dependency such as TWOi have little to no agency within this structure and are thus contingent on it. The space environment is itself extreme and unruly and open to unpredictable and in principle unknowable events which any work must respond to.

Here we have looked at how a work can be emergent from this kind of situation by framing it as an open participatory structure. We return to this in Situation 4 where we look at other strategies for addressing the unique challenges of creating cultural projects in space, including extending the discussion of tragedy started here. 


\subsection{Situation 3}

This situation examines the role of external administrative bureaucratic and legal structures in the development of the Kicksat project and the impact they had on the development of the TWOi-project.

Straight after the re-entry off Kicksat 1, May 14, 2014 Manchester made an announcement that he planned to build Kicksat-2. It emerged that he had built 3 Kicksat deployer satellites during the Kicksat-1 development phase and re-development of these started immediately. Kicksat-2 was granted a new NASA-ELaNA award on February 6, 2015 with no set date. After the announcement of the KickSat 2 launch there was a long pause before the launch date was finally announced, which meant that effectively the KickSat-2 project did not progress at all during 2016. There were infrequent messages to the developer group about the delay and at the time the public information was that there was a hold up with radio licensing from the FCC.

\subsubsection{Radio Spectrum}

KickSat2 was conceived by Zachary Manchester and the team at Cornell University as a radio technology demonstration project. The scientific goal of the project was to test out new ultra low power Low Earth Orbit to ground station radio transmissions from the KickSat sprite satellites. The community use of the satellites, characterisation as personal satellites, and Kickstarter campaign in this situation can be seen primarily as a way of funding, promoting and enabling that project.

The radio transmitters embedded in the Sprite satellites were part of an embedded microcontroller, the TI MSP430 which has an on chip radio transmitter. The Sprites broadcast at a frequency of 437.240 MHz using a novel digital encoding schema based on Gold Codes. Gold codes are massively redundant mathematical streams of bits that can be identified if only a small part of the pattern is matched and are successfully used in many radio applications including GPS. This encoding enables a huge signal to noise ratio boost of $56 \mathrm{~dB}$ from the signal processing at the cost of greatly slowed transmission and decoding speeds. The TWOisatellite transmissions averaged 23 bytes per minute after de-coding. The signals are very low power and effectively below the noise floor. They have to be received with Software defined radio apparatus and the decoding is processor intensive, hard to achieve in real time and best achieved with post processing of recorded signals. 
This low power radio schema could be a key technology for pico-satellites as it makes it possible to massively reduce the antenna size, and power requirements of on board radio equipment for telemetry. In the case of the sprite it allows an LEO to Ground transmission using nothing more than a short $10 \mathrm{~cm}$ antenna, a capacitor for energy storage and a microcontroller based radio, all of which made a spacecraft with a mass of $4.2 \mathrm{gm}$ viable.

The KickSat experiment was therefore designed to test this transmission protocol from space as a proof of concept for future development of pico-spacecraft.

In common with all other satellite deployments, there are a large number of regulatory approvals that need to be granted before a satellite can be put on a manifest and launched into orbit. One of these is radio spectrum approval, and in the case of KickSat 1 this had been unproblematically granted by the FCC.

In 2015 Manchester made an application to the FCC for radio spectrum approval for 437.240 MHz for KickSat 2 but on May 26, 2016, the FCC sent a dismissal letter rejecting the application.

This refers to application, File No. 1288-EX-ST-2015, for an experimental authorization.You are advised that the Commission is unable to grant your application for the facilities requested. We are not able to approve your request due to concerns regarding the overall risk and uncertainties associated with your experiment. There are concerns about the trackability and operational impacts of these satellites (sprites), and of this type of satellite in general.

We acknowledge the work done by Cornell with NASA to mitigate potential impacts on NASA operations however there are other potentially affected operators. We believe there are still risks with international operators that exist and cannot be addressed in the limited time frame remaining.

We cannot conclude that any public interest of your experiment outweighs the risk of going forward with this experiment. (FCC, 2016)

The reason given in the letter is concern about trackability and operational impacts, and of the class of sprite satellite. While the application to the FCC was for a radio spectrum license, it was rejected on the basis of concerns about the sprites being untrackable debris. However the flight plan and technology of KickSat 1 was identical to KickSat 2 and KickSat 1 had it's radio application accepted by the FCC. In addition the Kicksat 2 had also passed a NASA 
Orbital debris assessment which concluded there was no risk to other spacecraft Treptow (2015).

Although they previously licensed pico-satellites of less that $10 \mathrm{~cm}$ from 2016 the FCC stopped licensing satellites smaller than Cubesats citing lack of trackability.

\subsubsection{Debris}

Since KickSat 1 and concurrent with the growing development of private space exploration, there had been a growing public concern about the increase in space debris.

Space debris consists of spacecraft or spacecraft parts that remain in orbit after a mission ends and includes satellites that have reached the end of their lives and discarded rocket stages. A 1966 Life magazine lists 234 pieces of space debris in orbit (Inc, 1966, p.29).

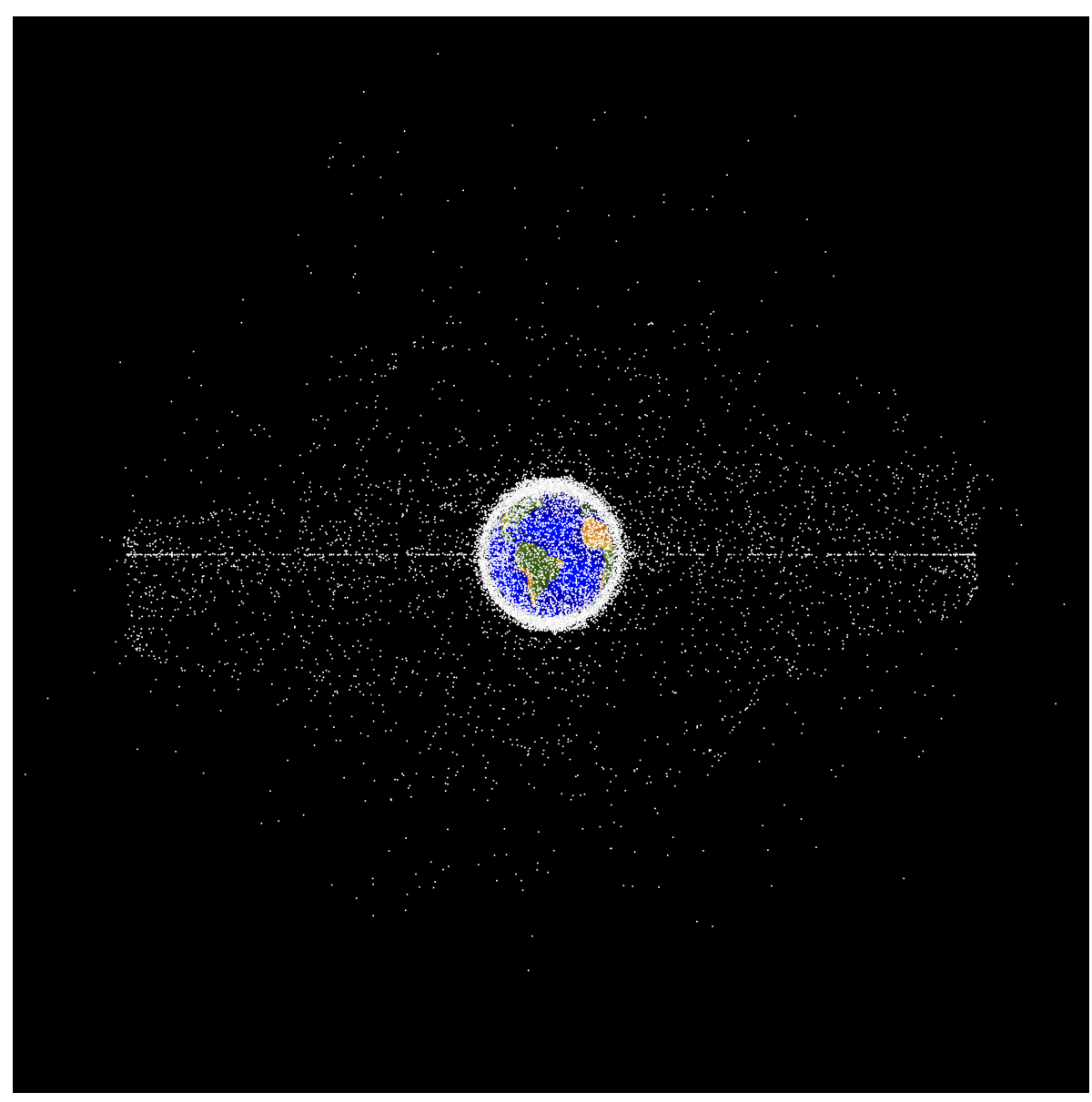

Figure 16: Orbital Debris Jan 12019

Note. Computer generated images of objects in Earth from GEO by NASA Orbital Debris Program Office. January 1, 2019. By O. NASA (2019). Public Domain.

\#+LATEX 
As of Feb 2020 the European Space Agency lists the following statistics (ESA, Feburary 2020)

\begin{tabular}{ll}
\hline Functional satellites & 2,300 \\
Mon-functional satellites & 3,200 \\
Space Surveillance Network tracked objects & 22,300 \\
Estimated breakups & 500 \\
Estimated objects $>10 \mathrm{~cm}$ & 34,000 \\
Estimated objects $1 \mathrm{~cm}->10 \mathrm{~cm}$ & 900,000 \\
Estimated objects $1 \mathrm{~mm}$ to $1 \mathrm{~cm}$ & $128,000,000$ \\
Total mass in orbit & 8800 tonnes \\
\hline
\end{tabular}

Table 7: Satellite Statistics

Due to the high velocity of objects in orbit, collisions with even small objects can cause catastrophic damage to a spacecraft, and these collisions have been observed. It was theorised by Kessler (1991) that a catastrophic cascade of collisions, and spacecraft break up could render space unusable. Mitigation strategies to minimise debris have only been relatively recently implemented, with NASA mandating mitigation strategies in 2001, ESA in 2002. There is no international treaty on space debris but, the UN Committee on Peaceful Uses of Outer Space published voluntary guidelines in 2007 after a series of discussions that started in 1993 (Rajapaksa \& Wijerathna, 2017). It has been modelled that Cubesats may lead to a worsening of the situation without strict mitigation strategies (Lewis, Schwarz, George, \& Stokes, 2014)]]. A recent test destruction of a satellite by the Indian Military has raised awareness of the potential for debris through acts of warfare in space (Johnson-Freese \& Burbach, 2019).

The issue has been referenced in cultural projects and its public profile was heightened with the release of the film Gravity (Cuarón, 2013), which depicted a Kessler collision cascade. Daan Roosegaarde created Space Waste Lab , an artwork which tracked space debris with laser beams (Space Waste Lab | Studio Roosegaarde, 2017). A similar work SPACEJUNK, which pointed twigs at space debris, was created by Bowen Bowen (2016). There is a rapidly growing field of space mitigation strategies, and for Cubesats guidelines include completion of an Orbital Debris Assessment Report and recommendations that Cubesats are released on trajectories that de-orbit in a reasonable amount of time Initiative (2017).

\subsubsection{Swarm}

There are jurisdictional issues at play here with no standardised international accreditation process. In practice each nation maintains it's own regulatory structure with rough alignment in blocks, and some international co-ordination at a treaty level. 
In 2017 satellite startup Swarm applied for a radio license from US radio regulator the Federal Communications Commission [FCC]. The license was for a constellation of satellites which were $3 \times 10 \times 10 \mathrm{~cm}$ or $1 / 3$ Cubesat size. In the rejection the FCC cited inability to track the satellites due to their size, creating a space debris issue.

In the absence of tracking at the same level as available for objects $10 \mathrm{~cm} \times 10 \mathrm{~cm} \times 10 \mathrm{~cm}$, and in the event of a conjunction with an operational spacecraft, the ability of operational spacecraft to reliably asses the need for and plan effective collision avoidance maneuvers will be reduced or eliminated. Accordingly, we cannot conclude that a grant of this application is in the public interest. Serafini (2017)

Swarm responded by ignoring the FCC and entering a launch contract with PSLV in India without US licensing. In 2018 FCC then prosecuted and fined Swarm \$900,000 USD. The launch was from India and outside of US jurisdiction, but the FCC claimed jurisdiction because Swarm was headquartered in the US ("FCC Fines Swarm \$900,000 for Unauthorized Satellite Launch", 2018). Subsequently the FCC released an enforcement advisory, restating their jurisdiction (FCC, 2018). Swarm eventually added KU band reflectors to their satellites improving visibility and tracking, paid the fine and were granted a license by the FCC.

\subsubsection{NTIA}

The refusal to license the sprite satellites radio only 2 days before the satellite was due to be delivered to NASA, effectively put the KickSat 2 project on hold. After much legal advice, a way forward was discovered. Radio spectrum management in the US is taken care of by the FCC for private companies and individuals. Federal agencies however, are generally licensed by the NTIA which is a separate licensing administration. They co-operate closely with the FCC and they generally provide co-coordinated decision making, but the NTIA is able to grant radio spectrum licenses independently Nichols (n.d.).

NASA as a federal agency routinely apply to the NTIA for their radio spectrum licenses rather than the FCC. This was the key to allowing the KickSat 2 project to move forward with a license.

The KickSat project was already scheduled to fly on a NASA space craft, had the launch funded by the NASA educational launch programme, and had been cleared through NASA's own Orbital Debris Assessment process. The solution to the FCC licensing issue was that 
KickSat 2 project was taken on by NASA as an official NASA project, applied for a license through the NTIA and was accepted, despite the FCC dismissal of the license. From this point on therefore KickSat 2 was effectively on paper an official NASA project.

\subsubsection{Summary}

In this situation we see the impact of external regulatory frameworks on two pico-satellite projects, Swarm and Kicksat/TWOi. In both cases the rationale for licence rejection by the FCC was the size of the satellites, which were below the established Cubesat form factor. There are two issues of jurisdiction here. The first is that the FCC is a communications commission and is not technically responsible for space debris assessments or regulation which are the domain of NASA, even though this is what is cited as the reason for the rejections. The second is that the Swarm launch in particular was from another sovereign state and the jurisdiction was claimed because of Swarm's headquarters.

With no binding international debris treaty currently in place, it appears that the FCC is taking the approach of using radio co-ordination regulation which is under binding international treaty, to extend its reach into debris regulation. As the number of space faring nations, launch vehicles and satellite operators increases, the swarm prosecution can be seen as a public statement by the FCC of it's future intent. It can only be hoped that binding international treaties take the place of this ad hoc regulation schema.

The contestation of regulation in small satellites and commercial launch, including active disregard of regulation and regulator over-reach can be seen as a consequence of the shift in conditions of space access. These conditions have changed from being bound and regulated by national space administrations, to a more fluid international and commercial environment. Swarm have taken a chance on contesting the existing ad hoc framework to establish precedent, and have been rebuked. Kicksat has returned to the umbrella of the previous national paradigm and come under the umbrella of NASA and NTIA. This new positioning again moves the personal spacecraft further into the background, returning to a public institution rather than being legally even individual in the sense of a commercial corporation.

For the TWOi-project these episodes again demonstrate entanglement but this time with a regulatory environment and its global evolution. Lastly the use of radio regulation for debris mitigation, demonstrates the entanglement at an institutional level of the physical and the informational. 


\subsection{Situation 4}

TWOi and tragedy Describes how the TWOi artwork uses tragedy to create engagement with space

This situation examines responses in the TWOi-artwork to the issue of extreme physical inaccessibility of the satellite to Earth based audiences. There are two main approaches are examined, firstly a tragic framing narrative and secondly the orbital path of the satellite and it's diagrammatic representation. These are contrasted with secondary techniques of anthropomorphism and exhibits of launch ephemera.

\subsubsection{Visibility}

The key aesthetic problem of using a satellite as material for an artwork is that the satellite is physically inaccessible to the audience, is $350 \mathrm{~km}$ above the surface of the Earth and travelling at high speed. The remoteness of the satellite and its extremely small size makes it invisible to direct perception by the audience. This lack of visibility hinders engagement with the artwork.

It is possible to make directly perceivable orbital objects and these are visible satellites which are directly discernible by earth based observers. This is true for the ISS itself, Iridium satellites, the new Star-Link constellation, the early Echo project etc. [expand], all of which can be seen crossing the night sky if you know where and when to look. Satellite prediction software such as Heavens Above or Gpredict offers detailed time, azimuth and elevation data for many satellites. Two art satellite projects - Humanity Star, and Trevor Paglen's work use this effect to create visible satellite artworks, and I myself have designed an unrealised visible satellite artwork 'Beatnik' in the past.

\subsubsection{Identification}

I first witnessed identification with a satellite whilst watching the visible pass of the ISS with my daughter Hannah at Whanganui Observatory. She was restless and uninterested at first, but when she learned that there were 3 astronauts living in the space station, effectively in an orbiting house, she became fascinated and as the ISS came into view I saw her gain a strong connection with the orbiting space station, an identification and a shift of view point so that she imagined herself looking down. It seemed that while the visibility of the satellite was the visual confirmation of its existence, the identification was with the astronauts on board. 
The TWOi-satellite, at 2cm across, was not visible from Earth, and its only Earth bound signal is a radio transmission. The radio signal is heavily mediated by technical apparatus and requires specialised equipment to recover the message. A high gain steerable motorised and computer controlled antenna, a software defined radio decoder and a computer processor with software decoder are all required. Due to the way the signal is encoded using gold codes, these signals when received are not decodeable in real time and require significant post processing to retrieve.

\subsubsection{Ephemera}

During the various exhibitions ephemeral evidence of the satellite, videos of the rocket launch, the development board, the tracking robot, and displays of location maps with real time updated tracking information as well as various media feeds, were displayed in the gallery and online as supporting media.

In gallery presentations of TWOi at the Engine Room (2015) and Thomas King Observatory (2018) the development board, which is almost identical to the actual satellite, was placed in a display cabinet consisting of a scientific scale, adjusted to show a negative weight. In Travares Strachan's ENOCH (2015), a similar strategy was employed with an identical display sculpture to the one in orbit presented to audiences at LACMA.

The TWOi exhibitions also featured a robot satellite tracking antenna, which points at the satellite as it makes a pass overhead, allowing it to track potential radio transmissions. The antenna has a dual purpose in the artwork which is to physically demonstrate the direction of the satellite. In the Engine Room 2015 TWOi-requiem piece, the robot traced out the historical path of the satellite during it's 2014 flight. This pointing strategy is used in two earth based works mentioned in the section on debris, Space Waste Lab (Roosegaarde 2017) and SPACEJUNK (Bowen 2017). The pointing robot, and these two artworks are driven by tracking software which converts a satellites TLE into azimuth and elevation data. The tracking software itself was shown in TWOi exhibitions, as a world map with the real time location of the satellite and it's track. In TWOi the launch videos were shown alongside real time and historical telemetry data. Lastly in one of the display videos, a 3D CGI generated animation of the Kicksat Cubesat was shown which depicts the sprite satellite being ejected from the deployer. The animation is cut in with real footage from the flight of the launch and ISS docking. The environment of space with its direct and predictable lighting, fits well with de- 
fault $3 \mathrm{~d}$ aesthetics and this strategy was also used in the gallery presentation of Strachan's ENOCH (Strachan, 2017).

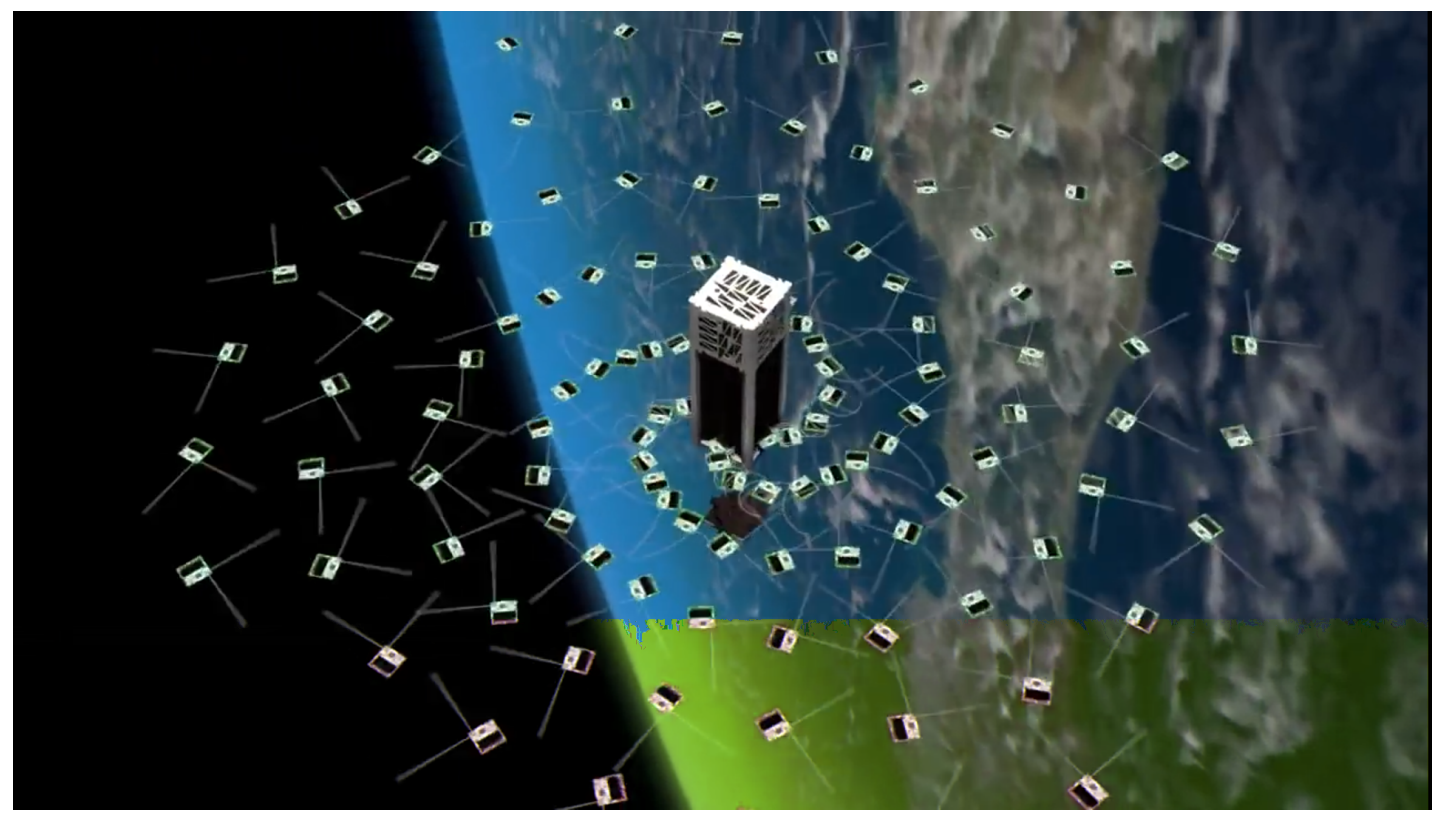

Figure 17: CGI depiction of KickSat

Note. CGI depiction of Kicksat showing sprite satellite deployment.Manchester (2011). With permission of Zac Manchester.

\subsubsection{Storyworld}

However all of the preceding examples do not themselves constitute direct experience of the orbiting space craft and must be considered as supporting materials. The primary attempt to address distance was the TWOi-storyworld narrative device. Herman describes a storyworld as an implicit or explicit world that is created by narrative, in whatever form, but never actualised (Heinen, Sommer, \& Herman, 2009). In the TWOi-artwork a storyworld is created by descriptions in the media, direct conversation, and printed collateral texts and this acts as a framing device for the artwork. In this storyworld, information is responsible for gravitation, and the process of deleting it will be active in the physical universe, making the spacecraft lighter and allowing it to float into a higher orbit.

In publicity texts, talks and in conversation with participants of the TWOi-artwork, a character called TWOi is described (TWOi-character) who is identified as the satellite (TWOisatellite). 
TWOi will then begin a descent towards Earth where it is destined to burn up within a few days. As it orbits it will collect information from its sensors filling its memory before deleting everything. It is like a tragic hero who having finally made it into the heavens is pulled back down to Earth in spiral orbit and eventual re-entry burn up. To escape this untimely end the satellite deletes information trying to ascend by forgetting. (Priest, 2014)

\subsubsection{Anthropomorphism}

In this framing description TWOi-character is clearly anthropomorphised and represents a human character. A similar anthropomorphic technique was used with Sputnik who as a character voices "Let's drop the formalities. You can call me Beep Beep." (Siegelbaum, 2012) This anthropomorphisation is possible because of the common systems description that correlates a human or living being with a computer system that was developed in early descriptions of cybernetics by Wiener (1961).

In common with the cybernetic description of a computer or an animal, the system is reduced to a minimum of inputs, outputs, feedback loops and processes, with internal complexity hidden from view. From a physical point of view the TWOi-satellite is technically a spacecraft that has been reduced to a minimum of complexity. It has an energy source, the solar panel, a body, a PCB, a processor, a memory, some sensors, a gyroscope and compass, and a means of communication, the radio. In an anthropomorphised sense TWOi is a reduced human, with a body that is a material object that takes care of sustaining energy flows, some sensors that can perceive the external world spatially, a processor that represents a brain, a memory that allows for the experience of time, and a radio transceiver that allows for communication with other sentient entities.

The reduced body is placed into the reduced environment of Low Earth Orbit. TWOi exists in microgravity in free fall across a geodesic, and has little interaction with the material world beyond occasional collisions with gas molecules in the ultra sparse atmosphere of Space. Space is here defined as Low Earth Orbit above what is called the Karman line which is the height at which aerodynamic lift generated by the sparse atmosphere is equal to the escape velocity. This height of $\sim 83.6 \mathrm{~km}$ marks the end of the area of Earthly dynamics and is normally approximated up to $100 \mathrm{~km}$ (Holley, 1968).

The external connections with matter are reduced and what are foregrounded are the en- 
ergetic flows. TWOi's direct inputs from the world come through incident light on the solar panel, the interaction with Earth's magnetic field through the compass, description of free fall through the accelerometer, and outputs through the radio transmitter. TWOi absorbs sunlight which drive processes, and outputs $437 \mathrm{MHz}$ radio messages, a little infrared heat from the processor and reflects green from the circuit board.

TWOi's internal life is equally reduced. The program running on TWOi grabs data from the sensors, fills the memory with a data stream until it is full, and then deletes it. TWOi counts the amount of data collected, calculates a measure of its complexity, records a simple count of sessions and transmits this meta data back to Earth through its radio. While the TWOisatellite models a cybernetic reduced human system, this in itself does not create a reason for a human audience to have any kind of emotional attachment to it.

Nass, Steuer, Tauber, and Reeder (1993) describe a process by which humans come to anthropomorphise computers and term this ethopoeia extending the classical term for rhetorical impersonation ${ }^{22}$. Nass then established empirical evidence for the creation of anthropomorphic responses to computers in which human users actually behave as though the computers they are interacting with are human. This set of experiments relied on specific forms of communication and response and hence took place in an interactive setting.

Unlike Sputnik which was both visible and audible through radio, in TWOi-artwork there was no possibility of either perception or interaction with the satellite. For Nass then, these forms of anthropomorphism cannot be responsible for identification in this case and we must return to the content of the narrative itself.

\subsubsection{Tragedy}

The transgressive conceit of the artwork is that this process of collecting and forgetting, accumulation and erasure is somehow responsible for a shift in orbit. Wiener neatly defines this boundary; "Information is information, not matter or energy. No materialism which does not admit this can survive at the present day." (Wiener, 1961, p.132)

The space craft is physically on a spiral trajectory back down to Earth and it is presumed in the narrative that TWOi-satellite is doomed to a fiery end with the material world inexorably

\footnotetext{
${ }^{22}$ In classical rhetoric ethopoeia is a technique by which a speaker voices another person's views or feelings in order to persuade an audience of an argument. This relies on the success of believable characterisation by the speaker. Nass extends the concept to examine the process by which humans are willing to accept computers as human interlocutors, using the term ethopoeia.
} 
asserting itself and standard physics prevailing.

This unfortunate destiny is used in the artwork to explicitly position the satellite as a tragic hero in the classical sense. Here the hero attempts to cross from the earthly realm to the heavens. TWOi-satellite leaves the bounds of the Earth and succeeds in entering the heavens conquering gravity and embarking on an epic journey from Earth, to the space station, the mortal's outpost in the heavens. But once it flies free into autonomous orbit tragedy befalls TWOi and it is pulled back down to Earth by the weight of information or perhaps worldly knowledge in a biblical fall from grace.

The tragedy in the narrative is that TWOi performs a heroic act, only to be defeated by an unforeseen situation. This is the tragic error or hamartia as described by Aristotle in the poetics, which confirms TWOi's humanity, and leads to TWOi's demise. Aristotle describes hamartia as "a representation of an action which is serious, complete and through the arousal of pity and fear effecting the katharsis of such emotions." (Aristotle, 1997)

From the point of view of the human audience the tragic narrative evokes pity and fear which allows the audience to emotionally identify with TWOi, and empathise, experience and internalise the Weight of Information in a visceral way. This is process is called katharsis and creates engagement for the audience.

Eagleton systematically critiques the idea of the tragic hero in classical tragedy preferring the term tragic protagonist, in which the individual and her actions are not separated. The tragic hero, he argues emerges much later with the modern conception of human personality.

Further he gives examples of classical dramatic actions without even protagonists. "In a kind of anti-humanist thought experiment, one could imagine the possibility of an action without them, rather as an artist might draw without colour." (Eagleton, 2002, p.77)

This however poses a problem for audience identification as the arousal of pity and fear are dependent on some kind of identification with a protagonist.

"Aristotle claims in the Poetics that pity and fear are intertwined. We pity others for what we fear may happen to ourselves, and those incapable of the one feeling are thus impervious to the other as well." (Eagleton, 2002, p.154)

In Turri (2015) the katharsis of classical tragedy is related to the psychological processes of transference in which a traumatic experience is put under critical reflection by creating distance from it. Paradoxically the possibility of engagement is created by the critical psy- 
chological distance in the transference process. For Turri it is mimesis that creates dramatic enactment which allows the spectator to emotionally engage with the tragic character, while tragedy promotes a distancing similar to transference. "I argue that it is the fruitful dynamic between close emotional identification and sympathetic detachment which represents the quintessential meaning of tragic katharsis." (Turri, 2015)

The tragedy gets its potency by exposing the subject to an emotionally painful situation whilst maintaining a critical distance that creates a vantage point of safety. Moving beyond the individual Eagleton highlights a separate political reading of tragedy.

Philippe Lacoue-Labarthe sees Greek tragedy as a politics of emotion, insisting that pity and fear are political rather than psychological notions. Pity refers to the social bond, whereas fear refers to the danger of its dissolution. (Eagleton, 2002, p.154)

In TWOi the goal of identification with the satellite was to reference for the audience political aspects of information through solidarity with the satellite, and here Lacoue-Labarthe suggests a mechanism using tragedy.

\subsubsection{Line}

In Walter de Maria's Vertical Earth Kilometer, a one kilometer long brass rod is buried end on so that only the 2 inch diameter end is visible from the surface De Maria (1977). In this work the visible part of the work is very small and the work is primarily experienced as a conceptual artwork. The power of the work consists in it's actual material reality and extension beneath the Earth's surface for $1 \mathrm{~km}$. It would not work as a purely conceptual work being little more than a coin stuck to the floor. The monumental scale of the work, the small proof of it's existence, combine to create a powerful mental image and affect. TWOi-artwork in the same way uses a small amount of evidence in the ephemera, a monumental scale in the orbit and combines this with the TWOi-storyworld to create identification. 


\section{THE WEIGHT OF INFORMATION}

April 14th May 7th 2014
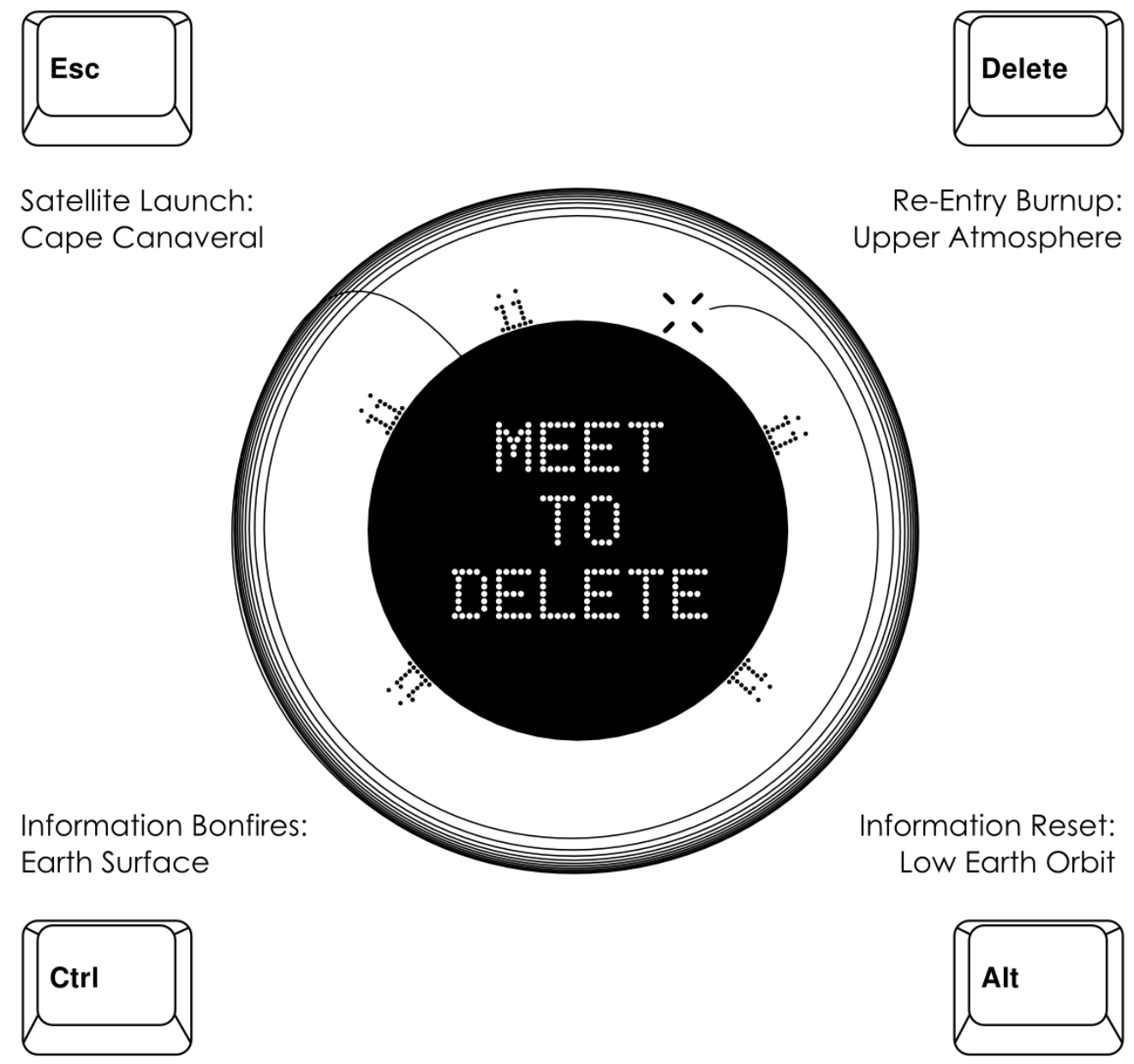

http: / / julianpriest.org/twoi

Figure 18: TWOi poster 2014

Note. The Weight of Information poster from 2014. By Julian Priest.

Low Earth Orbit provides a similarly large scale for TWOi-artwork with the Earth's mean radius $6371.0 \mathrm{~km}$ and LEO $\sim 350 \mathrm{~km}$ giving a diameter of $13442 \mathrm{~km}$, a size that dwarfs the 
human scale and in romantic era art parlance this vastness qualifies Low Earth Orbit as an example of Burke's sublime. Most of this orbital trajectory is parallel to the Earth's surface and in the plane that most of human life takes place in. Considered as drawing TWOi-artwork traces an orbital line around Earth, rather like Eagleton's description of how 'an artist might draw without colour'. This imaginary line is represented in the satellite tracks visible on the monitor and also referenced in the artwork publicity. It forms a secondary part of the mental image in the conceptual artwork, with the Earth represented as a black circle with TWOi's spiral orbital trajectory around it from launch to burn up.

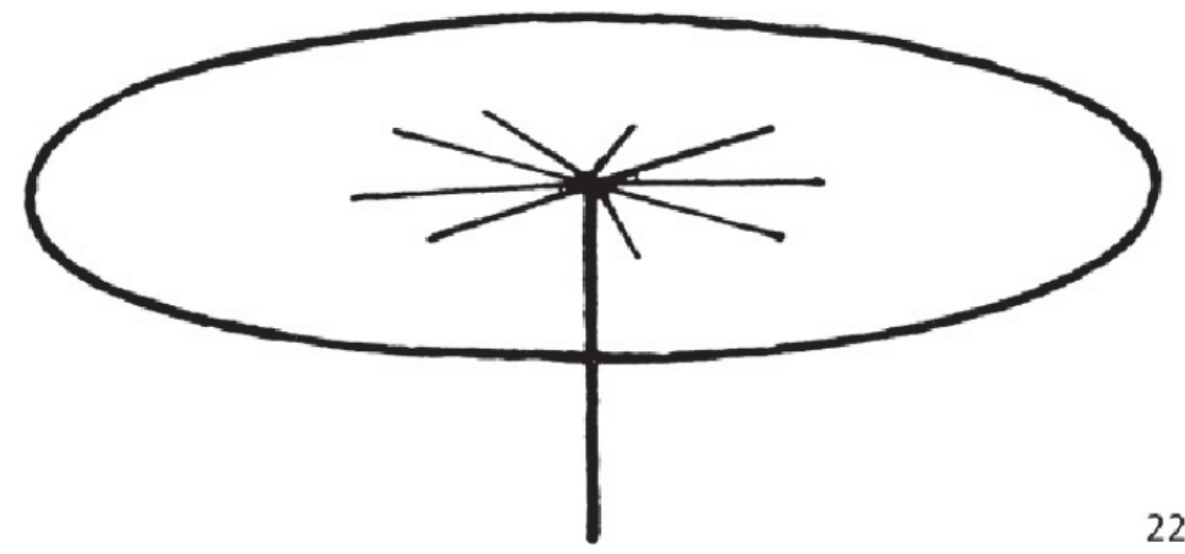

Figure 19: Paul Klee Horizon image

Note. Wagrecht: Der Horizont als Vorstellung. From Padagogisches Skizzenbuch. By Klee (1925). Public domain.

From point to Line. the point is not dimensionless but an infinitely small planar element, an agent carrying out zero motion, i.e. resting. Mobility is the condition of change. Certain things have primordial motion. The point is cosmic, a primordial element. Things on earth are obstructed in their movement; they require an impetus. The primordial movement, the agent is a point that sets itself in motion (genesis of form). A line comes into being. The most highly-charged line is the most authentic line because it is the most active. In all these examples the principal and active line develops freely. It goes out for a walk, so to speak, aimlessly for the sake of the walk. (Klee, 2012, p.193)

Paul Klee's famous expression taking a line for a walk is elaborated here in 1956 in his writings on the basics of form and composition. Here Klee almost describes the orbital envi- 
ronment in contrast to "Things on earth are obstructed in their impetus" and the agent is a point that sets itself in motion. The sentence "an agent carrying out zero motion i.e resting" equally well describes the free fall of an orbital geodesic. This diagram of the horizon and its relation to gravity, also shows the orbital path, and its orthogonal relation to the gravitational field, at right angles to Walter de Maria's Vertical Earth Kilometer.

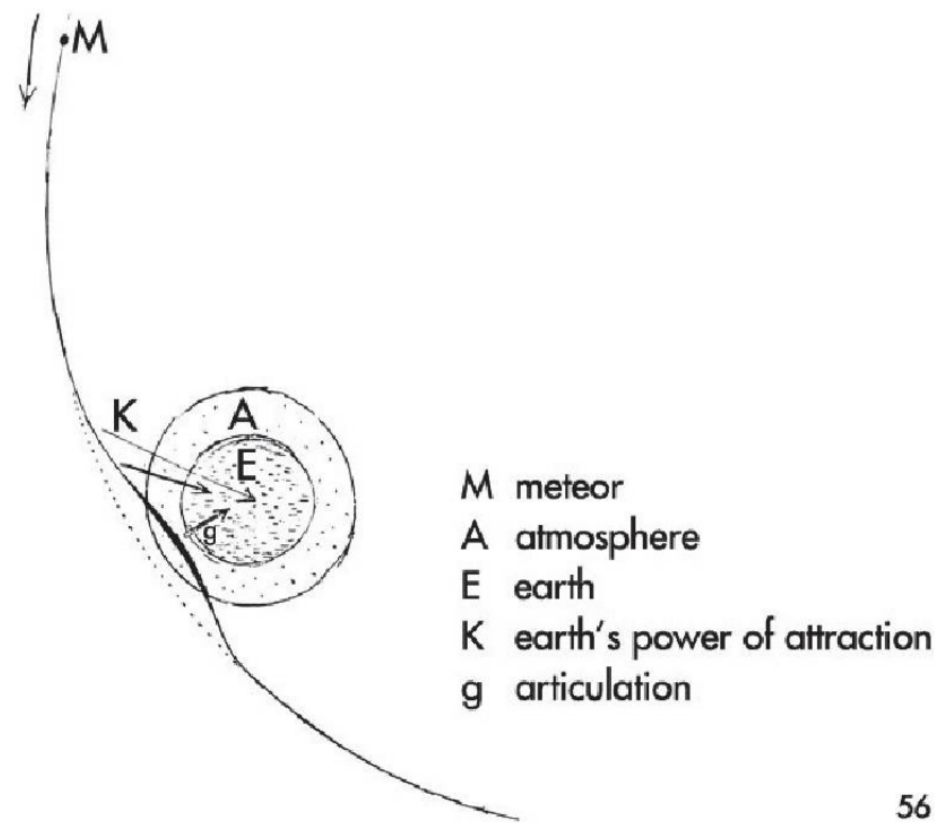

Figure 20: Paul Klee Meteor trajectory

Note. Kosmisch und atmosphärisch kombiniert. From Padagogisches Skizzenbuch. By Klee (1925). Public domain.

Later in the same text Klee considers the orbital trajectory of a meteor, and its "articulation" by the atmosphere. It is clear that in these descriptions of line as free agent, the orbital relation to gravitation, and articulation of the orbit in the presence of the atmosphere, in Klee's theory of the basis of form and composition describes the orbital dynamics of TWOi-satellite and a plausible connection with aesthetic dimension of TWOi-artwork.

Klee develops these ideas in his techniques of composition, but In Richard Long's iconic work A Line Made By Walking, Klee's idea of a line is translated directly into a physical form (Long, 1967). Long walked backwards and forwards across a grass field pressing compressing the grass in straight line which he then photographed to create an image Roelstraete (2010). Similarly the orbital trajectory of TWOi-satellite is inscribed in the orbital track displayed through the tracking software to form an imprint of the satellite's trajectory which becomes 
part of the TWOi-artwork.

The tangential nature of the orbital track to the Earth's gravitational field places the satellite into a parallel trajectory to that which humans experience when walking on Earth. Gatt \& Ingold in describing a study on the ethnography of walking, investigates the social aspects of walking and describes walking abreast in conversation with a companion as an ideal form of social interaction which is less confrontational than face to face conversation (Gatt \& Ingold, 2013). Ingold and Lee further relate walking abreast together to the oral narrative tradition of story telling (Coleman \& Collins, 2007). This form of parallel companionship they contend does not involve interaction in that there is no bridging operation, rather they describe a relation of correspondence like "..the movement of pedestrians moving along together" (Gatt \& Ingold, 2013, p.143).

The image of / moving along together/ and hence the concept of correspondence appears as a key concept early on in the history of Spaceflight in the naming of the first human made orbital satellite Sputnik. The Russian word спутник (Sputnik) from old church Slavonic literally means travelling companion, or fellow traveller, originally a religious term. Used for satellite it is a shortening of (sputnik zemlyi) or travelling companion of the Earth and was used by Soviet engineers in the phrase Простейший спутник-1 (Prosteishiy Sputnik - PS-1) - Simplest Satellite-1. There is a separate word in Russian попутчик (Poputchik) which also means fellow traveller and had been used in post revolutionary Russia to describe fellow travellers in the sense of sharing politaical ideology and the concept of fellow traveller was used in the McCarthy era to attack people with left leaning political tendencies. The difference in colloquial usage is that sputnik is a travelling companion, and poputchik is someone who happens to be someone who is going the same way.

The description of an artificial orbital satellite as a companion traveller was coined by the famous supremacist artist Kazimir, Malevich in 1920.

\footnotetext{
"The Suprematist machine, if it can be put that way, will be single-purposed and have no attachments. A bar alloyed with all the elements, like the earthly sphere, will bear the life of perfections, so that each constructed Suprematist body will be included in nature's natural organisation and will form a new Sputnik; it is merely a matter of finding the relationship between the two bodies racing in space. A new Sputnik can be built between Earth and Moon, a Suprematist Sput-
} 
nik equipped with all the elements that moves in an orbit, forming its own new path." (Shatskikh, 2014)

Here the figure of the satellite is positioned as a third character between the Earth and moon. At the time Malevich wrote this, the fellow traveller was a current theme and Rawlinson (2013) relates it to the sublime and the extreme expeditionary culture of early 20th century, the modernist literature of Eliott, and traces its history back to early Christian imagery where it appears as the soul of Christ. In each of these a strong emotional bond and identification is formed between the narrator and the fellow traveller.

Travares Strachan's /ENOCH/realises the character of the spiritual fellow traveller directly by placing a canopic jar with a bust of Lawrence, the first African American astronaut, in a stable orbit around Earth (Strachan, 2017). The canopic jar references 3 separate spiritual traditions and was blessed in a Shinto ceremony and recognized as containing Lawrence's soul.

TWOi-character by contrast is in an unstable orbital tension between the heroic escape from the gravitational pull of the earth and a tragic fall back down to earth.

\subsubsection{Summary}

The primary aesthetic difficulty in satellite art is the physical remoteness of the satellite from potential audiences. In TWOi-artwork this is exacerbated by the technical difficulty of non real-time transmission of data from the artwork. In the artwork the distance is addressed by different methods of creating an emotional identification between audience and artwork. These appear in the artwork materials as a narrative and an orbital diagram, which use engagement, anthropomorphism, a tragic narrative and correspondence. Anthropomorphism stemming from a cybernetic description of the satellite is not considered to be functional in this case. The tragic narrative is examined and related to psychological transference. The orbital path of the satellite is considered as a drawing, and placed in an art historical perspective with precursor space art. This is related to the anthropological theory of correspondence and the fellow traveller archetype that links back to pre orbital space art. 


\section{Discussion}

This research set out to understand how space art projects are enabled by new developments in the space industry through analysis of the production of the TWOi satellite artwork, and its position within the enabling Kicksat picosat project.

In the field review it was found that while cultural aspects were important in the development of the space exploration (Groys), and there has been a potential for peaceful use of space, there have been no dedicated autonomous art satellites before 2013. This was attributed to difficulties of access to orbit for individual artists.

In the first situation, the Kicksat project could provide this access this with a personal satellite delivered into orbit for a reduced cost under new conditions of space access. It was shown that, rather than low cost new launch platforms, it was lower total project cost that was important in this respect. The idea of a personal satellite was then critiqued in the light of the complex sets of relationships necessary for launch.

In contrast the enabling technologies were found to be interfaces (Bratton), form factors and boundary objects between the project and the larger launch infrastructure (Leigh Star). This critique appears to reduce the personal to a set of interface conditions produced through convergence with infrastructure (Chun), in contradiction to traditional autonomous artistic authorship.

In the second section the launch evolution was examined and the project infrastructure that is designed to eliminate contingency from launch, was shown to produce contingency through dependency - again working against individual agency. Situation 3 showed that the 2014 failure of the Kicksat satellite produced a community of practice and this was described in terms of protention and retention (Gell and Husserl). This suggested that the TWOi artwork be seen as a network of perchings distributed in continuous time, which was found to fit with the participatory nature of the work (Bishop).

The single particle cause of the deployment failure however showed a highly discontinuous time manifold, that rejects the smooth manifold of contingencies present in Husserl's model. This discontinuity was then supported in an interpretation of events using agential realism that highlights the role of intra-action and entanglement (Barad). This microscopic version of entanglement was found to mirror the macroscopic entanglement of social relations present in the launch and project infrastructures around the project (Hodder).

Crucially the dynamic present in both versions of entanglement has the potential to recover 
personal agency and artistic authorship. In the third situation the impact of external regulation was examined with three main findings. Firstly the regulatory environment is in flux as new entrants and form factors come on line. Second that radio spectrum regulation is being used to control physical space debris due to a lack of international treaty co-ordination, and thirdly that the Kicksat project moved away from being an independent private project to being a NASA project as a result of changes in spectrum regulation policy. In the last situation a range of artistic strategies were examined that address the physical separation of audience from satellite artwork of which tragedy (Eagleton) engendering psychological transference, and (Klee) line engendering correspondence (Ingold) are identified as possible techniques, whereas cybernetic anthropomorphism was rejected in this case.

The core tension in this research is between the personal and the infrastructural. The idea of a using a satellite as an artwork implies autonomous authorship by the artist, and yet the infrastructure which enables a satellite to be launched into orbit is heavily structured and controlled. At the outset it was proposed that the personal art satellite was enabled by the new conditions of access to space provided by commercial launch providers and reduced cost. While this may have an effect, I find that it is standardised small form factors and interfaces that facilitate ride sharing, that are currently the enabling factors. The boundary created by the interfaces creates a small area of autonomy within a much larger launch infrastructure, and this delimits a personal space that creates the possibility of artistic agency. Further the interface provides the structure and limits within the boundary which constitute Chun's personal through a process of convergence. This process is demonstrated in the Kicksat project's use of the Cubesat, and similarly in Hujon Song's personal satellite project. A similar process is at work in the TWOi project and it was this that primarily allowed an order of magnitude cost reduction through another layer of ride sharing. In this case the boundary objects were not physical specifications but were de-materialised as software encapsulation and the online platforms, GitHub and Kickstarter.

The full extent of the scale of the launch infrastructure becomes visible only when there are delays and disruptions. The first of these is the launch itself where the TWOi-project was caught up in a series of dependencies involved in the launch. Later delays caused by radio spectrum regulation and debris issues in situation 3 similarly expose the influence of the geopolitical policy framework. The action of this enormous set of infrastructural relationships is best described by Hodder's dynamic concept of entanglements, with internal 
features of Leigh Star's boundary objects and Bratton's interfaces.

The temporal discontinuities due to the launch infrastructure environment did diminish in importance as the satellite was deployed, and once in orbit a new set of relationships were produced. The satellite was also open to the harsh environment of outer space, where its materiality was highlighted and even the actions of single particles can have large consequences. There is a rolling dynamic present here too, and a discontinuous version of this is best described by the microscopic entanglement of Barad's agential realism.

This infrastructural analysis combined with the contingency of external forces in the space environment, limits the scope of the personal to the extent that it poses a problem for the possibility of artistic agency and authorship. Barad and Hodder's theories of entanglement taken together can show how both microscopic and infrastructural entanglements both structure an event, and provide a limited range of possibilities for its development, at each instant. This dynamic present recovers a space for artistic agency based on the material object of the satellite. The synthesis of infrastructural and microscopic entanglement begun here could be developed further with respect to the agency of the satellite.

The secondary theme concerns the artistic forms employed in TWOi. The key difficulty in orbit is the physical distance from audience or participants, and tragedy and a process of transference was used to bridge this gap. As a participatory artwork TWOi was open to the unfolding of events, and is best described as Gell's network of perchings. While tragedy was used in the TWOi storyworld, it's efficacy in structuring remote events was dramatically demonstrated in the failure of the 2014 deployment, where the event fostered a community of practice that engaged with it. While this strategy, as well as the others described, do bridge the distance to orbit, this is not to say that a more interactive direct two way communication would not provide greater engagement or artistic freedom in future.

The research is limited in that it covered only a part of the TWOi project. Four other situations covering the successful 2018 launch, the speculative physics experiment on board the satellite, the participatory installation of 2019, and 2015 gallery installation were omitted due to space constraints. In addition the retrospective nature of the case study meant that an exploratory methodology was used, and this has produced material across a wide range of fields, each of which is worthy of further examination in it's own right.

The TWOi project in common with the few other art satellites, demonstrates that it is possible to use satellites as material for artworks, and that this is currently feasible using existing 
space access methods. The field review highlighted the small number of extant art satellites, and a more detailed comparative study at this early stage in the field of art satellites would be worthwhile. Further cultural use of space for art satellites would be a valuable contribution to the peaceful use of space and should be encouraged. In the wider context the current regulatory environment with respect to space debris and radio spectrum is inconsistent internationally, and further work on multi-lateral solutions is needed.

This research has some practical implications for artists and cultural producers working with satellites. While chipsats like the Kicksat Sprite are extremely low cost, given the current developing regulatory stance on debris and trackability, they are unlikely to be easily licensed in the near future. By contrast the emergence of the Cubesat as a de-facto standard following it's large uptake, makes it a good choice of form factor. Careful consideration should be taken at the outset to minimise dependencies created by the launch choice including ride share partners and regulatory environment. Dedicated Cubesat launches should be chosen if possible, and ones with simplified regulatory environments and short timescales, such as those being offered in NZ may be preferable. Care should be taken with the detail of the relationships that enable the launch as these structure the project to a large degree. Space is a hazardous and difficult environment to operate in, and project should be designed with failure, redundancy and alternative potential outcomes in mind. The physical remoteness to orbit is a challenge for the artist, and as discussed in the field review, visible satellite artworks are likely to draw criticism. In the case of TWOi the work was been a conceptual artwork, supported by tragedy, correspondence and ephemera, of which tragedy was shown to be effective, but direct radio transmission or interactive communication could be future directions to be explored. 


\section{Conclusion}

In conclusion it is acknowledged that this thesis is limited to a partial treatment of a single art satellite project and hence provides a series of starting points for further work, rather than definitive conclusions. These conclusions should therefore be considered as interim results. To return to the main research question then, the conclusion of this thesis is that Space Art projects are enabled by new developments in the space industry. The thesis makes recommendations primarily for the art satellite rather than the wider field of space art. The prime enabling mechanism is the setting of interfaces, form factors and clear boundaries that enable ride sharing and therefore miniaturisation of satellites.

The new commercial launch arrangements while producing a reduction in overall cost for launch, perform a secondary role. The chipsat does provide an order of magnitude reduction in cost and improvement of accessibility through extreme miniaturisation and de-materialisation of interfaces. However it is impacted heavily by the current state of space debris regulation, and an increase in dependencies that make it problematic for future artistic use. It is hoped that further policy work on a multi-lateral approach to space debris will remedy this, but at present the Cubesat is recommended as preferred platform.

The secondary conclusion is that the tension between the personal and the infrastructural implicit in the launch infrastructure place an apparent limit on artistic agency in satellites. It appears that this may be resolved by further exploration of a synthesis of different concepts of entanglement from agential realism and material studies.

Thirdly art has played an important role in the development of space exploration but is under-represented in the peaceful use of space with no dedicated art satellite launched before 2013. This is so marked as to suggest a historical hostility to cultural work in space and why this should be so is a topic for future research. It is hoped that the new level of accessibility to orbit and increased direct public engagement in space will improve the situation.

Lastly the environment of orbit provides severe difficulties of accessibility and contingency for the production of artworks. Here strategies of participation, tragedy, ephemera and correspondence have been reviewed, with tragedy shown to be effective in producing identification between satellite and audience. Direct live two way interactive communication with a satellite is suggested as a future direction for research.

The dream of spaceflight has cultural roots which can be traced back to antiquity. The impact of artists is visible in the development of space exploration, and Sputnik by design and 
impact can be considered a cultural project. We have had to wait until new conditions of access to orbit made the launch of Hujon Song's OSSI-1, ArtSat, TWOi \& TWOi 2.0 and Orbital reflector possible. It is hoped that Travares Strachan's Enoch that is still in orbit in 2020 will be joined by an artistic fellow traveller in the very near future. 


\section{References}

\section{References}

Ada.net.nz. (2013, March). ADA - Aotearoa Digital Arts Network Dunedin Symposium. Retrieved 2021-01-11, from http://www.ada.net.nz/events/symposia/dunedin -symposium-programme/

Ada.net.nz. (2014). Ada.net.nz. ADA. Retrieved 2021-01-18, from http://www .ada.net $. \mathrm{nz} /$

Alves, P. M. S. (2008, October). Objective Time and the Experience of Time: Husserl's Theory of Time in Light of Some Theses of A. Einstein's Special Theory of Relativity. Husserl Studies, 24(3), 205-229. Retrieved 2020-05-14, from https://doi .org/ 10.1007/s10743-008-9039-1 doi: 10.1007/s10743-008-9039-1

Amsat. (2014, June). ARTSAT1:INVADER CubeSat - Cosmic Poem | AMSAT-UK [Blog]. Retrieved 2020-06-05, from https://amsat-uk.org/2014/06/26/artsat1invader -cubesat-cosmic-poem/

Aristotle. (1997). Poetics (New Ed edition ed.; M. Heath, Trans.). London ; New York, N.Y: Penguin Classics.

Artdesign.unsw.edu.au. (2015, August). Energies in the Arts Conference | UNSW Art \& Design. Retrieved 2021-01-11, from https://artdesign.unsw.edu.au/whats-on/events/ energies-arts-conference

Artsat. (2011, August). ARTSAT PROJECT1:INVADER. Retrieved 2020-06-05, from http:// artsat.jp/

Aurisicchio, M., Bracewell, R., \& Hooey, B. L. (2016, June). Rationale mapping and functional modelling enhanced root cause analysis. Safety Science, 85, 241-257. Retrieved 2020-06-21, from http://www . sciencedirect.com/science/article/pii/ S0925753515003513 doi: 10.1016/j.ssci.2015.12.022

Barad, K. (2007). Meeting the Universe Halfway: Quantum Physics and the Entanglement of Matter and Meaning. North Carolina: Duke University Press.

Bassa, C., \& Dijkema, T. J. (2019, March). Satellite observations obtained with the Dwingeloo radio telescope. Retrieved 2021-01-17, from https://charon.camras.nl/public/ satellites/

Beck, P. (2018, April). The Humanity Star. Retrieved 2020-06-05, from https : //web . archive 
.org/web/20180401114038/http://www. thehumanitystar.com/

Bishop, A. P., Van House, N. A., \& Buttenfield, B. P. (Eds.). (2003). Digital library use: Social practice in design and evaluation. Cambridge, Mass: MIT Press.

Bishop, C. (2012). Artificial hells: Participatory art and the politics of spectatorship. London ;: Verso.

Bowen. (2016). SPACE JUNK. Retrieved 2020-06-25, from https://mattress.org/ archive/index.php/Detail/Collections/1417

Bowker, G. C., \& Star, S. L. (1999). Sorting things out: Classification and its consequences. Cambridge, Mass.: MIT Press.

Brand, S. (n.d.). WholeEarth button. Retrieved 2021-01-12, from http: //sb . longnow .org/ SB_homepage/WholeEarth_buton.html

Bratton, B. H. (2015). The stack: On software and sovereignty.

Broeze, F. (2002). The globalisation of the oceans: Containerisation from the 1950s to the present. St. John's, Nfld: International Maritime Economic History Association.

Bureaud, A. (2014). Meet to Delete! Galerie Up, Bruxelles | annick bureaud. Retrieved 202101-25, from http: //www . annickbureaud . net/?p=873

Bureaud, A., Annick. (2015). It's a beautiful name for a satellite. In All Aliens (pp. 40-59). Valenciennes : Besançon.

Burrows, W. E. (2010). This New Ocean: The Story of the First Space Age. Random House Publishing Group.

Cees Bassa. (2019, March). The Sprites are alive! We just picked up transmissions at 437.24MHz with @radiotelescoop from the tiny Sprite satellites that were ejected from \#KickSAT2. Many thanks to @zacinaction for this great mission! The Sprites were ahead of \#KickSAT2 by as much as 5 mins. https://t.co/r8JNrwQrfy [Tweet]. Retrieved 2021-0117, from https://twitter. com/cgbassa/status/1109187588336824321

Celestrak.net. (2020). CelesTrak: Search of SATCAT. Retrieved 2020-06-02, from http:// www. celestrak.net/

Center, N. K. S. (2014, April). Dragon Spacecraft Separation. Retrieved 202101-18, from https://www.nasa.gov/downloadable/videos/dragon_spacecraft _separation.mp4

Chun, W. H. K. (2011). Programmed visions: Software and memory. Cambridge, Mass: MIT Press. 
Ciesielska, M., \& Petersen, G. (2013, September). Boundary object as a trust buffer. The study of an open source code repository. Tamara: Journal for Critical Organization Inquiry, 11(3). Retrieved 2020-06-17, from http : / tamarajournal . com/index . php/tamara/ article/view/164

Coleman, S., \& Collins, P. (2007). Locating the Field : Space, Place and Context in Anthropology. London, UNITED KINGDOM: Taylor and Francis. Retrieved from http:// ebookcentral.proquest. com/lib/vuw/detail. action?docID=487168

Coles, C., \& Jefferson, A. (1989). The Space Chronometer: An Orbiting Clock for Humanity. Leonardo, 22(2), 201-213. Retrieved 2020-06-04, from https: //www . jstor .org/ stable/1575232 doi: $10.2307 / 1575232$

Cooperation In Space. (n.d.). , 1.

Council, C. (1953, July). Convention for the Establishment of a European Organization for Nuclear Research | CERN Council. Retrieved 2020-07-04, from https : //council . web . cern.ch/en/convention

Crouch, T. D. (2000). Aiming for the Stars: The Dreamers and Doers of the Space Age. New York: Smithsonian Institution Scholarly Press.

Cuarón, A. (2013, October). Gravity [Drama, Sci-Fi, Thriller]. Warner Bros., Esperanto Filmoj, Heyday Films.

CubeSatShop.com. (n.d.). CubeSatShop.com - One-stop webshop for CubeSats \& Nanosats. Retrieved 2020-06-18, from https: //www . cubesatshop.com/

Currentsnewmedia.org. (n.d.). CURRENTS NEW MEDIA 2013. Retrieved 2021-01-11, from https://currentsnewmedia.org/festivals/currents-2013/

Dark Sky | Adam Art Gallery. (n.d.). Retrieved 2020-06-19, from https://www .adamartgallery.org.nz/product/dark-sky/

Dawson, M. (2014, December). Wanganui artwork heads to outer space. Retrieved 2021-0111, from https://www.nzherald.co.nz/whanganui-chronicle/news/wanganui -artwork-heads-to-outer-space/DZ3YK4JCKZOBLOQDYYM3KVEDTQ/

Deller, J. (2001). The Battle of Orgreave. Retrieved from https : / www . jeremydeller . org/ TheBattleOfOrgreave/TheBattleOfOrgreave_Video.php

De Maria, W. (1977). Vertical Earth Kilometer. Retrieved 2020-05-22, from https://www .atlasobscura.com/places/vertical-earth-kilometer

Department of Defense. (1967, August). DODGE Whole Disk Image. Retrieved 2021-01-13, 
from https://commons.wikimedia.org/wiki/File:First_color_image_of_the _earth_from_outer_space_(Dodge_Satellite).png

De Paulis, D. (2013, May). ISAW4 Hangout May 20, 2013. Retrieved 2021-01-11, from https://www . youtube. com/watch?v=oi-BRA75eUs

Díaz, E. (n.d.). Art and the New Space Age. , 17.

Diaz, E. (2018, May). We Are All Aliens. e-flux journal(91), 14. Retrieved 2021-01-22, from https://www.e-flux.com/journal/91/197883/we-are-all-aliens/

Diederichsen, D., \& Franke, A. (Eds.). (2013). The Whole Earth: California and the Disappearance of the Outside. Berlin: Sternberg Press.

Eagleton, T. (2002). Sweet violence: A study of the tragic. Oxford: Blackwell.

Earnshaw, R. (2016). Research and Development in Art, Design and Creativity. Cham: Springer International Publishing. Retrieved 2019-08-30, from http://link . springer . com/10 .1007/978-3-319-33005-1 doi: 10.1007/978-3-319-33005-1

Ellis, C., Adams, T. E., \& Bochner, A. P. (2010, November). Autoethnography: An Overview. Forum Qualitative Sozialforschung / Forum: Qualitative Social Research, 12(1). Retrieved 2019-08-26, from http: //www . qualitative-research.net/index .php/fqs/article/view/1589 doi: 10.17169/fqs-12.1.1589

ESA. (Feburary 2020). Space debris by the numbers. Retrieved 2020-06-25, from https:// www.esa.int/Safety_Security/Space_Debris/Space_debris_by_the_numbers

Esteves, D. (2019, April). Detecting the Sprites from KickSat-2 - Daniel Estévez [Blog]. Retrieved 2021-01-11, from https://destevez.net/2019/04/detecting -the-sprites-from-kicksat-2/

Ezell, E. C., \& Ezell, L. N. (1978). The Partnership: A History of the Apollo-Soyuz Test Project. National Aeronautics and Space Administration, Scientific and Technical Information Office.

FCC. (2016, May). Dismissal Without Prejudice generated [May 26 2016] Cornell University FCC ELS Correspondence Document [1288-EX-ST-2015]. Retrieved 2020-07-05, from https://fcc.report/ELS/Cornell-University/1288-EX-ST-2015/177330

FCC. (2018, April). Enforcement Advisory on Satellite Communications Licensing. Retrieved 2020-06-25, from https://www.fcc.gov/document/enforcement-advisory -satellite-communications-licensing

FCC fines Swarm $\$ 900,000$ for unauthorized satellite launch. (2018, December). Reuters. Re- 
trieved 2020-06-25, from https://www.reuters.com/article/us-usa-satellite -fine-idUSKCN10J2WT

Feyerabend, P. (1988). Against method (Rev. ed. ed.). London ;: Verso.

Finocchiaro, M. A. (1997). Galileo on the World Systems (First ed.). University of California Press. Retrieved 2020-06-03, from www.jstor .org/stable/10.1525/j.ctt1pp2jr

Freeman, L. (2019, February). Julian Priest. Retrieved 2021-01-11, from https://www.rnz.co.nz/national/programmes/standing-room-only/audio/ 2018682871/julian-priest

Fuller, M., \& Goffey, A. (n.d.). The unknown objects of object-orientation. , 13.

Galilei, G. (2016). Sidereus Nuncius, or The Sidereal Messenger (Second edition ed.; A. V. Helden, Trans.). Chicago: University of Chicago Press.

Gardiner, B. (2010, August). Alpha Geek: Hojun Song, DIY Satellite Builder. Wired, 18(9). Retrieved 2020-06-05, from https : //www . wired.com/2010/08/st_alphageek_song/

Gatt, C., \& Ingold, T. (2013). From Description to Correspondence: Anthropology in Real Time. In Design Anthropology: Theory and Practice. London, UNITED KINGDOM: Taylor \& Francis Group. Retrieved from https://ebookcentral-proquest -com.helicon. vuw. ac.nz/lib/vuw/reader. action?docID=1334415\&ppg=131

Gell, A. (1998). Art and agency: An anthropological theory. Oxford ;: Clarendon Press.

Godwin, R. (2016). William Leitch (UK ed. edition ed.). Apogee Prime.

Goldring, E. (1987). "Desert Sun/Desert Moon" and the SKY ART Manifesto. Leonardo, 20(4), 339. Retrieved 2020-07-05, from https://www.jstor.org/stable/1578529 ?origin=crossref doi: $10.2307 / 1578529$

Gorman, A., \& Roberts, A. (2019). Dr Space Junk vs The Universe: Archaeology and the Future. Cambridge, MA: The MIT Press.

Grōss, B. (2008). Art power. Cambridge, Mass: MIT Press.

Groys, B. (2018). Russian Cosmism. Cambridge, MA: MIT Press.

Guridi, R. (2019). A museum on the moon? The Moon Museum, exhibition space off limits. Revista de Arquitectura, 21, 244-248.

Hagler, G. (2013). Rocketmen. In G. Hagler (Ed.), Modeling Ships and Space Craft: The Science and Art of Mastering the Oceans and Sky (pp. 199-219). New York, NY: Springer. Retrieved 2020-07-05, from https://doi.org/10.1007/978-1-4614-4596-8_10 doi: 10.1007/978-1-4614-4596-8_10 
Heinen, S., Sommer, R., \& Herman, D. (2009). Narratology in the Age of Cross-Disciplinary Narrative Research. Berlin/Boston, GERMANY: De Gruyter, Inc. Retrieved 2020-0625, from http://ebookcentral.proquest.com/lib/vuw/detail.action?docID= 476043

Henderson, L. D. (2005). Duchamp in context: Science and technology in the Large glass and related works. Princeton, N.J. ;: Princeton University Press.

Hodder, I. (2012). Entangled: An Archaeology of the Relationships Between Humans and Things. Hoboken, UNITED STATES: John Wiley \& Sons, Incorporated. Retrieved 2020-04-07, from http://ebookcentral.proquest.com/lib/vuw/detail.action ?doc ID $=821899$

Holley, I. B. (1968, February). The Wind and Beyond. Theodore von Karman, Pioneer in Aviation and Pathfinder in Space. Theodore von Karman with Lee Edson. Little, Brown, Boston, 1967. viii + 376 pp., illus. \$10. Science, 159(3814), 517-518. Retrieved 2020-0422, from https://www. sciencemag.org/lookup/doi/10.1126/science.159.3814 .517 doi: $10.1126 /$ science.159.3814.517

Husserl, E. (1929). The Phenomenology of Internal Time-Consciousness. Indiana University Press.

Inc, T. (1966, August). Space Trash. Life Magazine, 29. Retrieved 2021-01-15, from https : // books.google.dk/books?id=_VUEAAAAMBAJ\&lpg=PA1\&hl=da\&pg=PA29

Infante, J. J. D. (2018, June). Inspiring Mexicans to "Imagine Amazing Things." In Space. Retrieved 2020-06-08, from https://slate.com/technology/2018/ 06/the-mexican-civil-space-agency-founder-on-inspiring-citizens-to -launch-satellites.html

Infante, J. J. D., Finn, E., Castro, F., Gasca, O., \& Lafuente, A. (2017). Ulises 1 - an art mission to space by the Colectivo Espacial Mexicano. Mexico: Center for Science and the Imagination at Arizona State University. Retrieved from https://csi.asu.edu/books/ ulises/

Initiative, N. C. L. (2017). NASA CubeSat 101: Basic Concepts and Processes for First-Time CubeSat Developers. NASA CubeSat Launch Initiative.

Ishikawa, K. (1990). Introduction to quality control /. London: Chapman \& Hall.

Isilaunch.com. (n.d.). Fully-serviced Launch Solutions. Retrieved 2020-06-18, from https://www.isilaunch.com/ 
Johnson-Freese, J., \& Burbach, D. (2019, July). The Outer Space Treaty and the weaponization of space. Bulletin of the Atomic Scientists, 75(4), 137-141. Retrieved 2020-06-25, from https://doi.org/10.1080/00963402.2019.1628458 doi: 10.1080/00963402.2019 .1628458

Jones, H. W. (n.d.). The Recent Large Reduction in Space Launch Cost. , 10.

Kac, E. (1988). SATELLITE ART: AN INTERVEW WITH NAM JUNE PAIK. Retrieved 2020-07-05, from http://www. ekac.org/paik. interview.html

KAHN, D. (2013). Edmond Dewan and Cybernetic Hi-Fi. In Earth Sound Earth Signal (First ed., pp. 93-105). University of California Press. Retrieved 2020-06-04, from www . jstor .org/stable/10.1525/j.ctt46n4hg. 12

Kessler, D. J. (1991). Collisional cascading: The limits of population growth in low earth orbit. Advances in Space Research, 11(12), 63-66. doi: 10.1016/0273-1177(91)90543-S

KickSat-gs. (2012). KickSat's GS mailing list. Retrieved 2021-01-18, from https://groups .google.com/g/kicksat-gs/

Kicksat-gs. (2014, May). KickSat Telemetry. Retrieved 2014-05-09, from https://docs.google.com/spreadsheets/d/1M1NL1sF3vQtV2r7gAmra1Z -I4v9N7m9Es1CBWOdi4E0/edit?usp=sharing

Kim, H. (2014). The Basement Satellite. Retrieved 2020-06-05, from https ://mydramalist .com/13269-the-basement-satellite

Kish, L. B. (2008). Weight fluctuations of information storage media. Retrieved 2021-01-12, from https://arxiv.org/abs/0805.4175

Klee, P. (1925). Pedagogical Sketchbook. London: Faber and Faber.

Klee, P. (2012). Paul Klee. Parkstone International.

Kovtun, E. F., \& Douglas, C. (1981). Kazimir Malevich. Art Journal, 41(3), 234-241. Retrieved 2019-10-19, from http://www.jstor .org/stable/776564

Kulu, E. (2020, April). Nanosats Database. Retrieved 2020-06-18, from https://www .nanosats.eu/

Leitch, W. (2012). God's Glory in the Heavens. Place of publication not identified: HardPress Publishing.

Lemonnier, P. (2012). Mundane Objects: Materiality and Non-Verbal Communication. Walnut Creek, UNITED STATES: Taylor \& Francis Group. Retrieved 2020-05-25, from http://ebookcentral.proquest. com/lib/vuw/detail.action?docID=1062360 
Lewis, H. G., Schwarz, B. S., George, S. G., \& Stokes, H. (2014). IAC-14-A6.4.1 AN ASSESSMENT OF CUBESAT COLLISION RISK. The International Astronautical Congress, 11. Long, R. (1967). A Line Made by Walking.

Lucian. (2014). Lucian (No. 14). Cambridge, MA: Harvard University Press.

Malevich, K. (1915). Black Square. Retrieved from https://archive.org/stream/ kazimir00male\#page/118/mode/2up

Malina, F. J. (1970). On the Visual Fine Arts in the Space Age. Leonardo, 3(3), 323-325. doi: $10.2307 / 1572339$

Malina, R. F. (1991/ed). In Defense of Space Art: The Role of the Artist in Space Exploration. International Astronomical Union Colloquium, 112, 145-152. Retrieved 2019-10-19, from https://www.cambridge.org/core/ journals/international-astronomical-union-colloquium/article/ in-defense-of-space-art-the-role-of-the-artist-in-space-exploration/ D105504B701DFD74647748319F33AE16 doi: 10.1017/S0252921100003894

Manchester, Z. (2011, October). KickSat - Your personal spacecraft in space! by Zachary Manchester - Kickstarter [Blog]. Retrieved 2013-05-31, from http:// www.kickstarter.com/projects/zacinaction/kicksat-your-personal -spacecraft-in-space

Manchester, Z., \& Peck, M. (2011, August). Stochastic Space Exploration with Microscale Spacecraft. In AIAA Guidance, Navigation, and Control Conference. Portland, Oregon: American Institute of Aeronautics and Astronautics. Retrieved 2020-04-13, from http://arc.aiaa.org/doi/10.2514/6.2011-6648 doi: 10.2514/6.2011-6648

Mathewson, S., \& 2017. (2017, February). India Launches Record-Breaking 104 Satellites on Single Rocket [Blog]. Retrieved 2020-06-14, from https ://www . space. com/35709 -india-rocket-launches-record-104-satellites.html

McLuhan, M. (1970, December). Education in the electronic age. Interchange, 1(4), 1-12. Retrieved 2020-06-02, from https://doi.org/10.1007/BF02214876 doi: 10.1007/ BF02214876

Mediarthistory.org. (2013, August). Media Art Histories 2013 programme. Retrieved from http://www.mediaarthistory.org/wp-content/uploads/2011/05/ MAH-programme-PRINT-1.pdf

Mieczkowski, Y. (2013). Eisenhower's Sputnik moment: The race for space and world prestige. 
Ithaca: Cornell University Press.

Mikel, J. (2014, May). KickSat Decay Prediction. Retrieved 2021-01-11, from https : //groups .google.com/g/kicksat-gs/c/hcwzNNY5uA4/m/HL_4-sWNZ6kJ

Mitchell, J. C. (2006, September). CASE AND SITUATION ANALYSIS. The Manchester School: Practice and Ethnographic Praxis in Anthropology, 21.

Murdin, P. (1991). Threat to Astronomy by Space Art. International Astronomical Union Colloquium, 112, 139-144. Retrieved 2019-10-20, from https://www.cambridge.org/ core/product/identifier/S0252921100003882/type/journal_article doi: 10 $.1017 / \mathrm{S} 0252921100003882$

Museum of Modern Art. (1959). The new American painting, as shown in eight European countries, 1958-1959. , 107. Retrieved from http://www .moma.org/calendar/ exhibitions/1990

NASA. (1967, November). ATS 3. Retrieved 2021-01-13, from http://library.ssec . wisc .edu/spinscan/images/1024cc/ATSIII_10NOV67_153107.jpg

NASA. (1972, December). Apollo 17: Blue Marble. Retrieved 2021-01-13, from http:// www . nasa.gov/image-feature/apollo-17-blue-marble

NASA. (2018, November). CRS-10 - Northrop Grumman. Retrieved 2021-01-16, from https://blogs.nasa.gov/northropgrumman/category/northrop-grumman/ crs-10/

NASA, J. K. (2018, November). Northrop Grumman Antares CRS-10 Launch | NASA Image and Video Library. Retrieved 2021-01-16, from https: //images . nasa.gov/details -NHQ201811170006

NASA, O. (2019, January). Orbital Debris GEO 2019. Retrieved 2021-01-15, from https:// orbitaldebris.jsc.nasa.gov/photo-gallery/_images/highresolution/ geo-2019-6144.jpg

NASA's Kennedy Space Center. (2014, April). Liftoff of SpaceX-3. Retrieved 2021-01-18, from https://www.nasa.gov/downloadable/videos/liftoff_of_spacex-3.mp4

Nass, C., Steuer, J., Tauber, E., \& Reeder, H. (1993). Anthropomorphism, agency, and ethopoeia: Computers as social actors. In (pp. 111-112). ACM. doi: 10.1145/ 259964.260137

Nichols, E. P. (n.d.). The Other FCC: The NTIA. Retrieved 2020-07-05, from http: //www . arrl .org/the-other-fcc-the-ntia 
Observatory, C. (2018, March). Space Place at Carter Observatory. Retrieved 2021-01-16, from https://en.wikipedia.org/w/index.php?title=Space_Place_at_Carter _Observatory\&oldid $=961801017$

O'Hare, M. (2015, July). Apollo-Soyuz: A cold war handshake in space, 40 years on $\mid$ New Scientist. Retrieved 2020-06-13, from https://www.newscientist.com/article/ dn27910-apollosoyuz-a-cold-war-handshake-in-space-40-years-on/

O’Neil, M., Muselli, L., Raissi, M., \& Zacchiroli, S. (2020, March). 'Open source has won and lost the war': Legitimising commercial-communal hybridisation in a FOSS project. New Media \& Society, 146144482090702. Retrieved 2020-07-05, from http://journals.sagepub.com/doi/10.1177/1461444820907022 doi: 10.1177/ 1461444820907022

Orbital Launch Summary by Year. (n.d.). Retrieved 2020-06-21, from http://www . spacelaunchreport.com/logyear.html

Outer Space Treaty. (1966, December). Retrieved 2020-05-31, from https://www. unoosa .org/oosa/en/ourwork/spacelaw/treaties/outerspacetreaty.html

Paik, N. J. (1984). Wrap around the World.

Pixelache. (2014, May). Pixelache | Meet to Delete (The Weight of Information). Retrieved 2021-01-16, from http://www . pixelache.ac/events/meet-to-delete-the -weight-of-information

Priest, J. (2012, December). Email.

Priest, J. (2013, September). Julian Priest, Ada 2013 Video. Retrieved 2021-01-11, from https://vimeo.com/76592363

Priest, J. (2014, March). Meet to Delete.

Priest, J. (2014-05-2014). Julianpriest.org: TWOi Status. Retrieved 2021-01-12, from https://julianpriest.org/project/twoi-status/

Ptarmigan. (2014, May). Ptarmigan. Retrieved 2020-12-15, from https://ptarmigan.ee/ events/meet-to-delete

Rajapaksa, C. R., \& Wijerathna, J. K. (2017, January). Adaptation to Space Debris Mitigation Guidelines and Space Law. Astropolitics, 15(1), 65-76. Retrieved 2020-06-25, from https://doi.org/10.1080/14777622.2017.1288513 doi: 10.1080/14777622.2017 .1288513

Rawlinson, M. (2013). Waste Dominion, White Warfare, and Antarctic Mod- 
ernism. Tate. Retrieved 2021-01-22, from https://www.tate.org.uk/art/ research-publications/the-sublime/mark-rawlinson-waste-dominion -white-warfare-and-antarctic-modernism-r1136827

Roelstraete, D. (2010). Richard Long: A line made by walking. London: Afterall Books. Rogers, L. (2008). It's ONLY Rocket Science An Introduction in Plain English (1st ed. 2008. ed.). New York, NY: Springer New York. doi: 10.1007/978-0-387-75378-2

Roll, P. G., Krotkov, R., \& Dicke, R. H. (1964). The equivalence of inertial and passive gravitational mass. Annals of physics, 26(3), 442-517. doi: 10.1016/0003-4916(64)90259-3

Rorty, R., Rorty, P. o. C. L. R., \& Richard, R. (1989). Contingency, Irony, and Solidarity. Cambridge University Press.

Ross-Nazzal, J. (2010, July). Détente on Earth and in Space: The Apollo-Soyuz Test Project. OAH Magazine of History, 24(3), 29-34. Retrieved 2020-06-04, from https : // academic.oup.com/maghis/article/24/3/29/967676 doi: 10.2307/maghis/24.3 .29

Ruggles, C. L. N. (2015). Handbook of Archaeoastronomy and Ethnoastronomy (1st ed. 2015. ed.). New York, NY: Springer New York.

Sagan, C., Sagan, L. S., \& Drake, F. (1972). A Message from Earth. Science, New Series, 175(4024), 881-884. Retrieved from http://www. jstor.org/stable/1733664

Saunders, F. S. (2000). Who Paid the Piper? : CIA and the Cultural Cold War. London: Granta Books.

Schon, D. A. (1983). The reflective practitioner: How professionals think in action. New York: Basic Books.

Serafini, A. (2017, December). DISMISSED-WITHOUT PREJUDICE. Federal Communications Commission.

Shatskikh, A. (2014). The cosmos and the canvas: Malevich at Tate Modern - Tate Etc| Tate. Tate Etc., Summer 2014(31). Retrieved 2020-04-05, from https : //www . tate . org . uk/ tate-etc/issue-31-summer-2014/cosmos-and-canvas

Siddiqi, A. (2008, July). Sputnik 50 years later: New evidence on its origins. Acta Astronautica, 63(1), 529-539. Retrieved 2020-06-26, from http://www.sciencedirect.com/ science/article/pii/S0094576507003542 doi: 10.1016/j.actaastro.2007.12.042

Siegelbaum, L. (2012, January). Sputnik Goes to Brussels: The Exhibition of a Soviet Technological Wonder. Journal of Contemporary History, 47(1), 120-136. Retrieved 
2020-06-27, from https://doi.org/10.1177/0022009411422372 doi: 10.1177/ 0022009411422372

Song, H. (2011, June). OSSI - NovaNano Launching Agreement. Retrieved 2020-06-07, from https://www . youtube.com/watch?v=enkgRR5c0tg

Song, H. (2014a, February). Impossible Is Expensive | Open Source Satellite Initiative Blog [Blog]. Retrieved 2020-07-05, from https://web.archive.org/web/ 20140222162140/http://opensat.cc/blog/uncategorized/impossible-is -expensive/

Song, H. (2014b, January). OSSI-1 Satellite Launch | Open Source Satellite Initiative Blog [Blog]. Retrieved 2020-06-30, from https://web.archive.org/ web/20140111224239/http://opensat.cc/blog/launch/ossi-1-satellite -launch/

Space Waste Lab | Studio Roosegaarde. (2017). Retrieved 2020-06-25, from https: //www .studioroosegaarde.net/project/space-waste-lab

Spaceweather.com Time Machine. (2014, April). Retrieved 2020-05-13, from https:// spaceweather . com/archive . php?view=1\&day=30\&month=04\&year=2014

Star, S. L., de la Bellacasa, M. P., Wakeford, N., Griesemer, J. R., Smith, B. C., Boland, R., ... Verran, H. (2016). Boundary Objects and Beyond: Working with Leigh Star (G. C. Bowker, S. Timmermans, A. E. Clarke, \& E. Balka, Eds.). Cambridge, Massachusetts: The MIT Press.

Starlink. (2020). Starlink. Retrieved 2020-07-05, from http://www . starlink. com

Strachan, T. (2017). ENOCH. Retrieved from https://www.lacma.org/lab/project/ enoch

Suvin, D. (1971). The Utopian Tradition of Russian Science Fiction. The Modern Language Review, 66(1), 139-159. Retrieved 2020-06-03, from https ://www . jstor.org/stable/ 3722475 doi: $10.2307 / 3722475$

TISA Workshop * Taksha Institute. (2013, May). Retrieved 2021-01-11, from https://www .taksha.org/divisions/TISA/events/ISAW4/

Treptow, J. (2015, September). Orbital Debris Assessment for KickSat-2 Mission per NASA-STD 8719.14A. NASA. Retrieved 2019-02-24, from https://apps.fcc.gov/els/GetAtt .html?id=171109\&x=.

Triscott, N., \& La Frenais, R. (2005). Zero gravity nevesomost': A cultural user's guide : The 
arts catalyst. S.l: Arts Catalyst.

Turri, M. G. (2015, April). Transference and Katharsis, Freud to Aristotle. The International Journal of Psychoanalysis, 96(2), 369-387. Retrieved 2020-05-20, from https://doi . org/10.1111/1745-8315.12243 doi: 10.1111/1745-8315.12243

Ucsusa.org. (2020). Satellite Database | Union of Concerned Scientists. Retrieved 2020-06-02, from https://www . ucsusa.org/resources/satellite-database

Verlinde, E. P. (2011, April). On the Origin of Gravity and the Laws of Newton. Journal of High Energy Physics, 2011(4). Retrieved 2018-12-20, from http://arxiv.org/abs/ 1001.0785 doi: 10.1007/JHEP04(2011)029

Vopson, M. M. (2019, September). The mass-energy-information equivalence principle. AIP Advances, 9(9), 095206. Retrieved 2021-01-12, from https://aip.scitation.org/ doi/full/10.1063/1.5123794 doi: 10.1063/1.5123794

Weber, S. (2004). The Success of Open Source. Harvard University Press.

Wiener. (1961). Cybernetics: Or Control and Communication in the Animal and the Machine (second edition edition ed.). Cambridge, Mass: MIT Press.

Winkler, M. G., \& Van Helden, A. (1992, June). Representing the Heavens: Galileo and Visual Astronomy. Isis, 83(2), 195-217. Retrieved 2020-06-03, from https ://www . journals .uchicago. edu/doi/10.1086/356110 doi: 10.1086/356110

Wirelesssummit.org. (2013, October). International Summit for Community Wireless Networks | 2-4 October 2013 | Berlin. Retrieved 2021-01-11, from https: //web . archive .org/web/20131006132446/http://2013.wirelesssummit.org/

Woods, A. (2019, October). Art to the Stars - an Astronautical Perspective on the Arts and Space. Retrieved 2020-06-05, from https: //thespaceoption.com/art-to-the -stars-an-astronautical-perspective-on-the-arts-and-space/

Woods, A. (2020). Arthur Woods Artist Biographical Information: AstroArtist - TerraArtist. Retrieved 2020-06-27, from https://www.cosmicdancer.com/arthur_woods_artist _biographical_information.php

Yourdon, E., \& Constantine, L. L. (1979). Structured design: Fundamentals of a discipline of computer program and systems design. Prentice-Hall, Inc. 


\section{Appendix A}

\subsection{Public Presentations}

1. Introduction

This appendix details all of the public presentations of The Weight of Information artwork and a timeline of the various associated events and appearances is presented here with brief descriptions and images.

The Weight of Information artwork was realised in two main parts; a satellite in orbit, and a series of events on the ground called Meet To Delete. The artwork has to date (2020) been formally presented three times, first in 2014 to coincide with the first TWOi satellite launch, second in 2015 in a standalone gallery installation, and lastly in 2018/2019 to coincide with the TWOi 2.0 launch.

In addition the work has been presented at a number of public events, conferences and festivals and these are included here as well as some media mentions. 
During the development period TWOi was presented at a variety of media art and technology conferences. The intention was to gain visibility for the project and to recruit galleries, groups and organisations that would host Meet 2 Delete events during the orbital period. These appearances also made connections with a community of other Space artists, curators, developers and practitioners some of whom would participate in later stages of the project.

a) ISAW4

May 20, 2013. NASA Research Park, Mountain View, CA, USA

TWOi was presented during a public online google hangouts session at the 4 th International Space Arts Workshop in a panel discussion with other space artists chaired by Richard Lowenberg (TISA Workshop « Taksha Institute, 2013). The session was hosted and archived by Daniela De Paulis (De Paulis, 2013). This was the first public presentation of the project. A follow up online panel was planned for November 26, 2013 with Trevor Paglen, Zachary Manchester and Hujon Song but a suitable time slot could not be found.

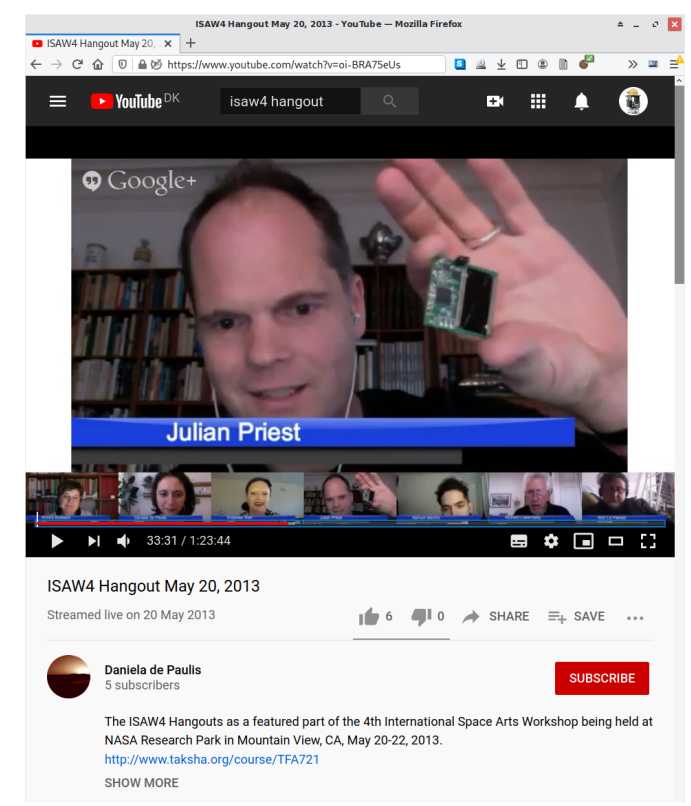

Figure 21: Screenshot of ISAW4 Online Event, 2013

Note. Screenshot of ISAW4 Event. By Julian Priest.

b) Currents 2013 
June 23, 2013. Santa Fe CCA - Cinemateque Studio, Santa Fe, NM, USA

A short video of the ISAW4 presentation was shown at Currents 2013 Santa Fe International New Media Festival (Currentsnewmedia.org, n.d.).

c) Ada Symposium 2013

September 13, 2013. C3 Church, 22 Vogel Street, Dunedin

Part of the TWOi project development took place in Italy and so this Dunedin presentation was given in a 12 minute pre-recorded video with q\&a session taking place live by Skype (Priest, 2013). The Ada symposium 2013 was aimed at artists, curators, practitioners and academics with a theme of Space, Network and Memory (Ada.net.nz, 2013).

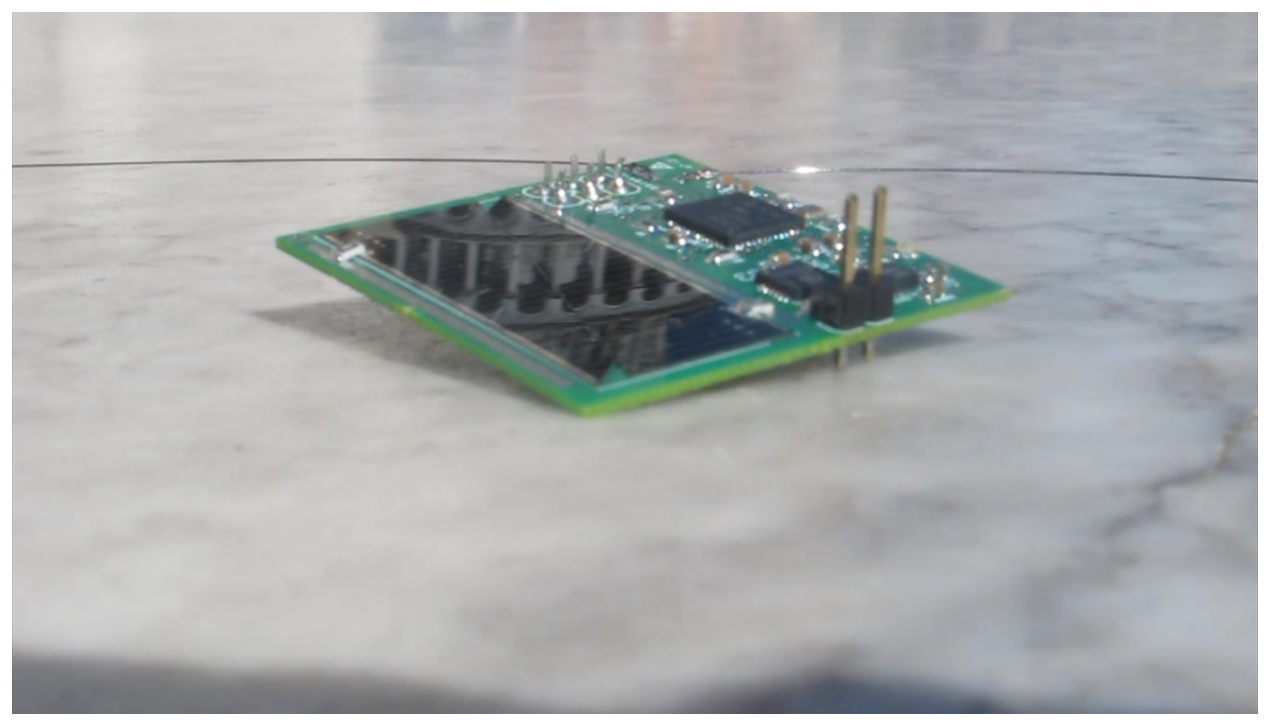

Figure 22: Ada Symposium 2013 Remote Video Presentation Still

Notes. Still from pre-recorded video presentation (Priest, 2013). By Julian Priest.

d) IS4CWN Wireless Summit 2013

October 2, 2013. C-Base, Rungestraße 2010179 Berlin-Mitte, Germany

A pico satellite workshop was presented at the International Summit for Community Wireless Networks (Wirelesssummit.org, 2013). The audience was largely technical with software defined radio experts, wireless network engineers and amateur satellite developers present. Peer commentary and feedback from this meeting improved some technical aspects of the development.

e) Renew: Media Art Histories 2013 
October 10, 2013. Stockholm School of Economics, Strelnieku street 4a, Riga Latvia TWOi was presented in a session on Techno-Ecologies at the Media Art History conference Mediarthistory.org (2013). The audience comprised largely art historians, curators, artists and practitioners. A call was made for curators to host the Meet to Delete events, and this generated interest from a number of organisers who would late stage events in support of the first launch.

3. 2014

This group of events were associated with the 2014 launch of the TWOi satellite. The initial events were local public events based in Whanganui, New Zealand to mark the launch of the satellite. The later event series were Meet to Delete events at different locations around the world. The Meet to Delete events were public events where participants were invited to bring some personal information to delete, and to share a story about what they were deleting. Suggestions were given about how to arrange the events and individual organisers tailored events to their own specific needs. Participants were instructed to delete information in solidarity with the satellite which was also repeatedly deleting information.

a) Launch Attempt 1 Event

March 16, 2014, 20:00 NZDT. Ward Observatory, St. Hill St, Whanganui, New Zealand

The TWOi satellite launch was announced in local media and a public event to witness the launch planned at the Ward Observatory, Whanganui, New Zealand Dawson (2014). The Ward Observatory is a historical observatory that is administrated by the Whanganui Astronomical Society and used for public astronomy evenings. The launch was scrubbed and an evening talk about the TWOi project given instead. 


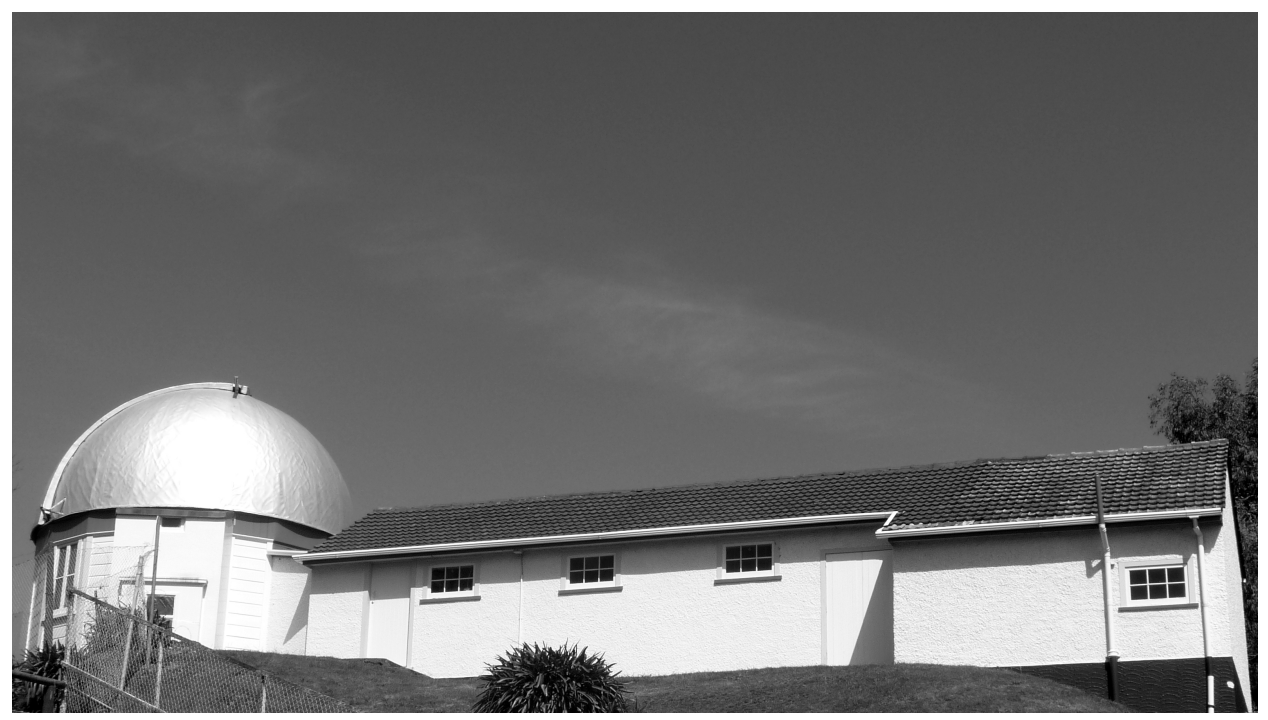

Figure 23: Ward Observatory

Note. View of the Ward Observatory.(Klajban, M. 2019) By Michal Klajban. Creative Commons Attribution-ShareAlike License.

b) Meet to Delete: Test Pilot

April 12, 2014 18:00 NZST. 37 Fortunatus Street, Wellington, New Zealand

Originally scheduled as the first Meet to Delete event, this was held in a private home in Wellington before the satellite had launched as a pilot to test the Meet to Delete process. Guests were invited to bring an item they would like to delete, share it with the group and then burn it in a bonfire. A projection showing the satellite position on the launchpad was screened.

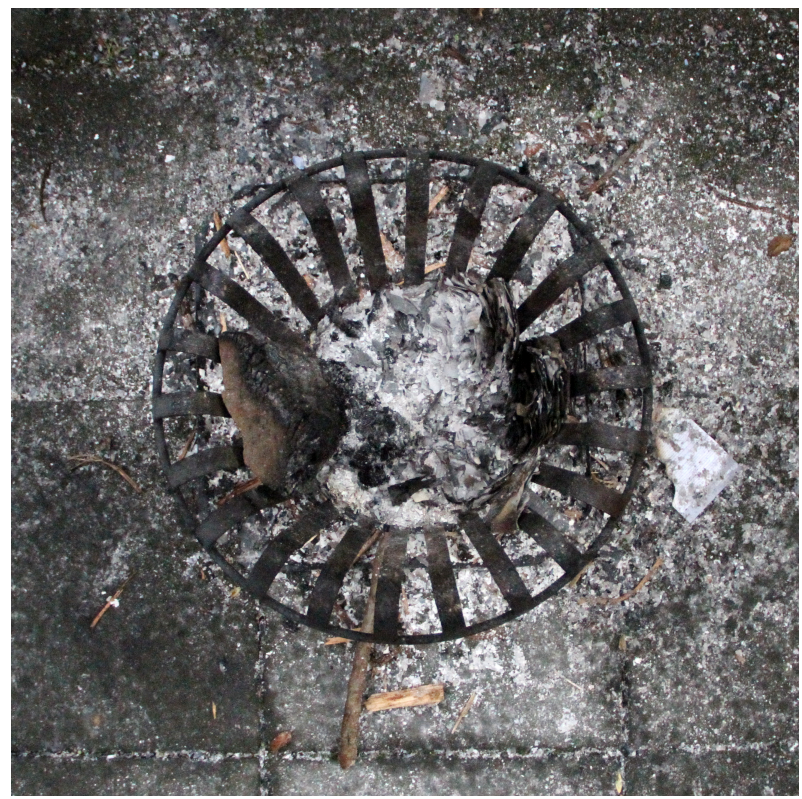


Figure 24: Meet to Delete Test Pilot Bonfire.

Note. Information ashes in the Meet to Delete brazier. By Sophie Jerram. With permission.

c) Launch Attempt Event 02

April 14, 2014 08:00 NZST. Ward Observatory, St. Hill St, Whanganui, New Zealand The second launch attempt was screened at the Ward Observatory in the morning and a school invited to attend. The launch was scrubbed due to a fire at NASA's Space Complex 41 ground tracking station and a talk was given instead. Doughnuts were served in recognition of a NASA tradition. 


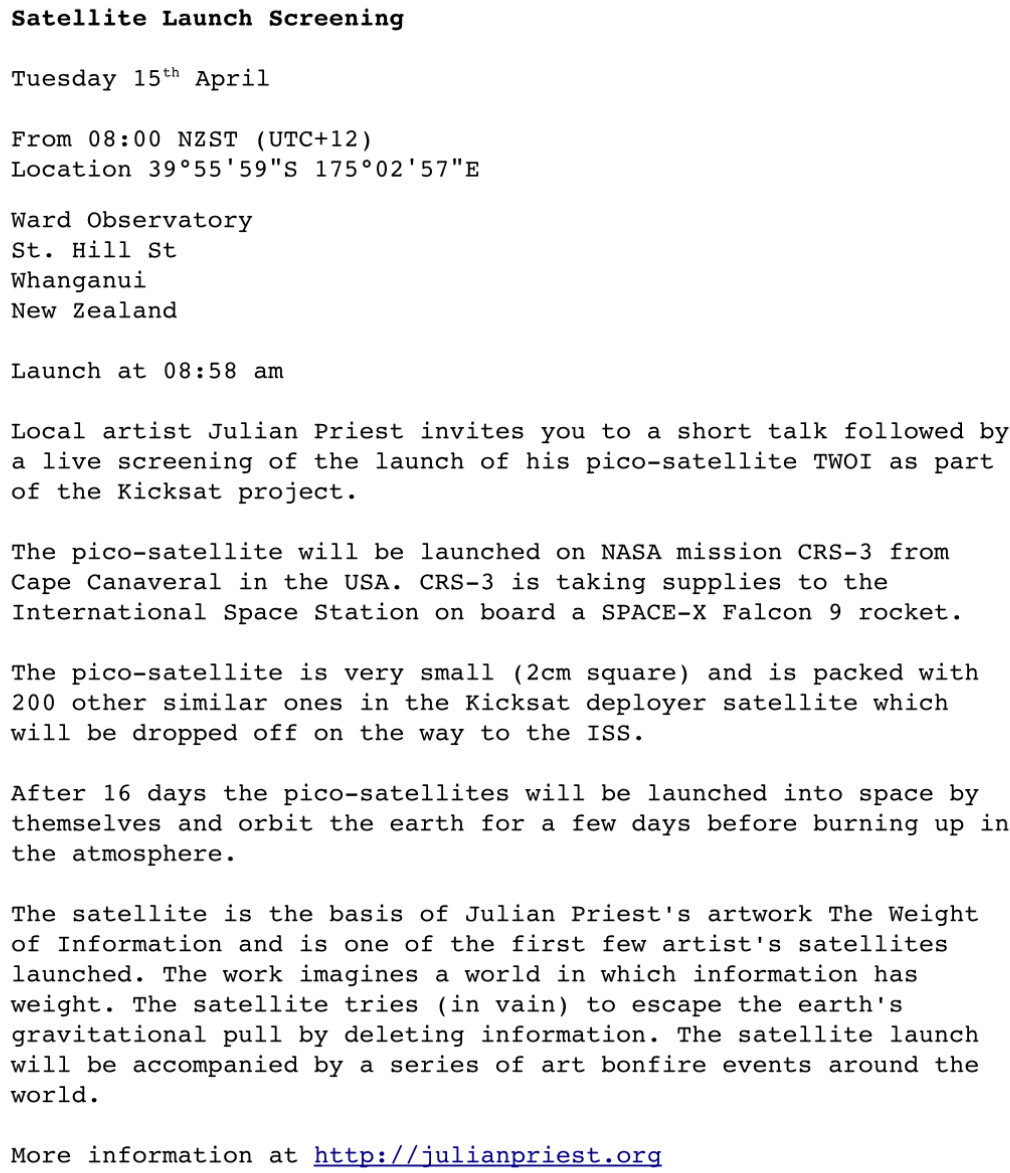

Figure 25: Launch Screening Invitation

Note. Letter sent out to a local school, inviting students to the event. By Julian Priest.

\section{d) Launch Screening}

April 18, 2014 06:00 NZST. Ward Observatory, St. Hill St, Whanganui, New Zealand

The successful launch screening was early in the morning and attended by a few friends, colleagues and close family. 


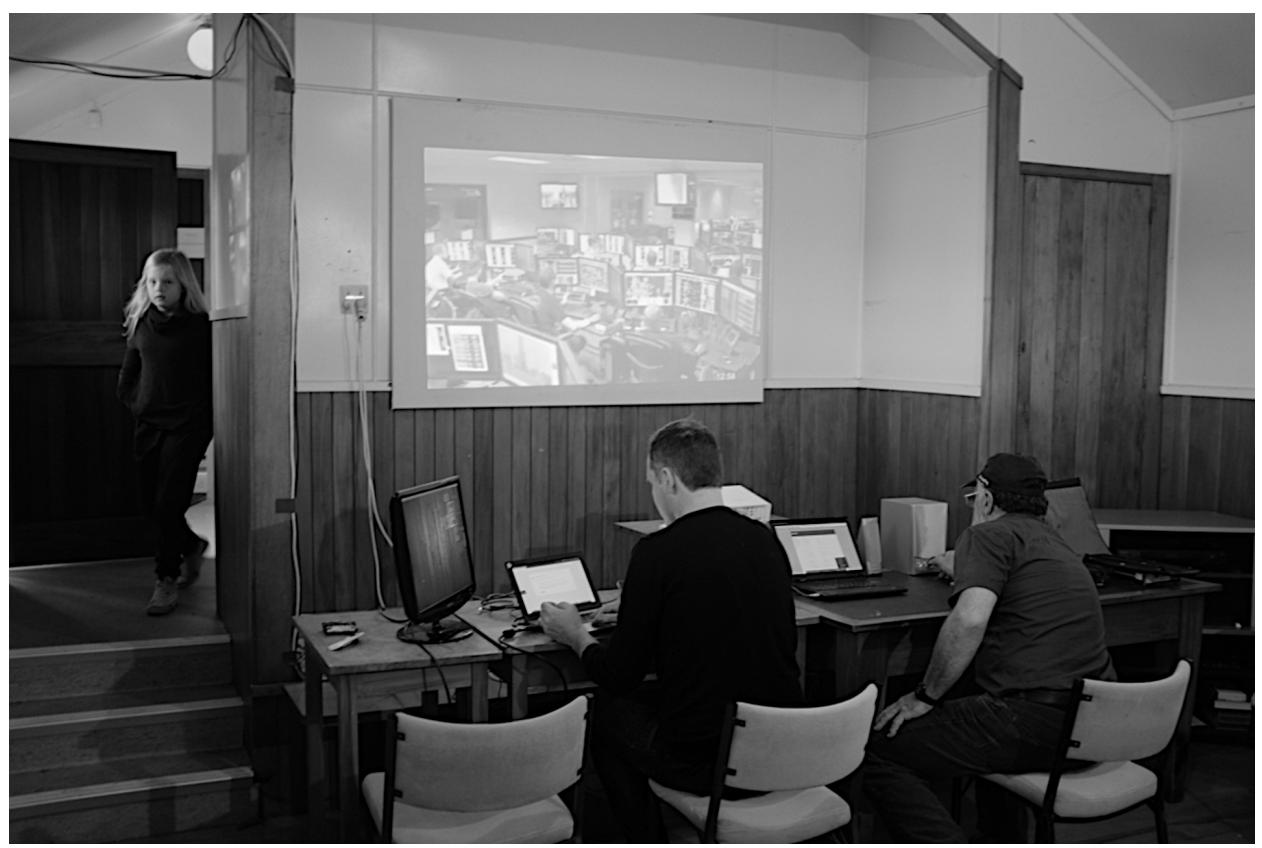

Figure 26: Successful Launch Screening

Note. Photograph of Whanganui Astronomical Society chairman, Ross Skilton, and Olga Priest at the launch screening of CRS-3. By Nicholas Twist. With permission.

e) Meet to Delete: Bruxelles

April 26, 2014 17:00 CEST. Up Galerie, 17 avenue Pauel Dejaer, 1060 Bruxelles, Belgium

Participants brought documents to destroy in a paper shredder. Meta data about what was deleted was recorded on a paper roll with a real time date of the orbit and telemetry displayed on a projector. The paper roll was shredded at the end of the event. A community discussion followed on the meaning of deletion and the weight of information (A. Bureaud, 2014).

It is difficult to decide and choose what to delete [in the framework of the Meet to Delete event], it is giving a weight to something that has no —or no more - value, that we want to get rid of and in a kind of a paradox giving it, for this moment, a central place. ... It takes time to delete, that is it takes our attention. There is a sort of paradox to isolate mentally oneself from the group to focus on the deleting process. 


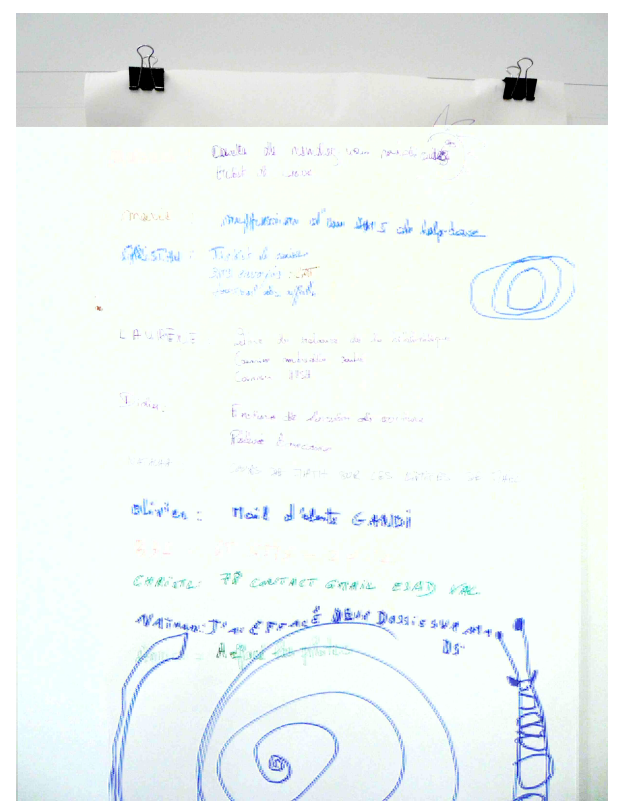

Figure 27: Meet to Delete, Up Galerie, Meta Data

Note. Up Galerie meta data list of deletions hand written on a roll. By Annick Buraud and Clarisse Bardiot. With permission.

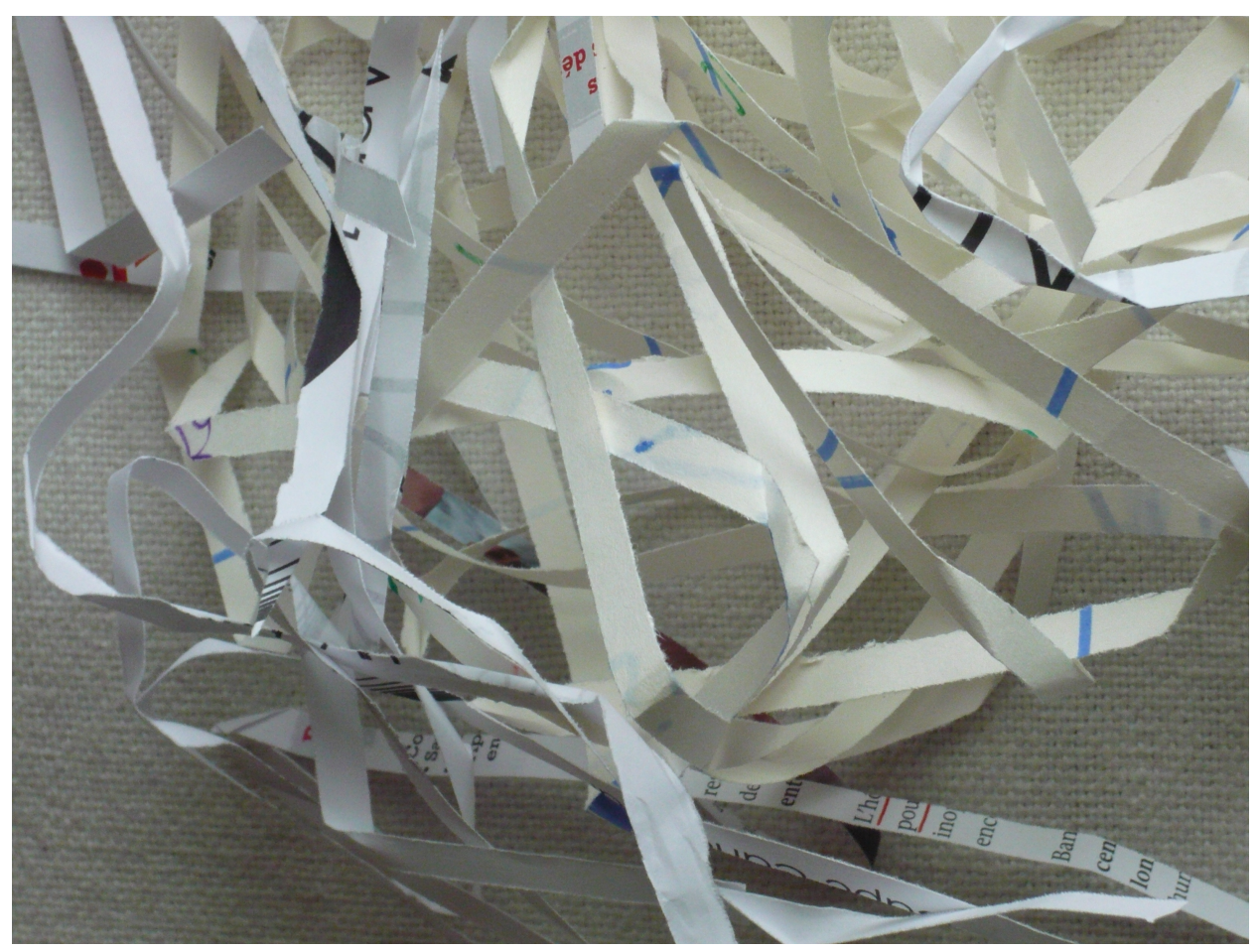

Figure 28: Meet to Delete, Up Galerie, Deletions

Note. Up Galerie shredded paper roll. By Annick Buraud and Clarisse Bardiot. With permission.

f) Meet to Delete: Receive and Recycle 
May 4, 2014 CEST. Szigethalom, 2315, Hungary

A Kicksat project participant Janos Tolgyesi organised an event in response to the Meet to Delete open call. In this event documents were burned in a group of 9 burners and the reflected light directed to towards the satellite in order to help illuminate its malfunctioning solar panels. The burners were arranged in the pattern of a Falcon 9 rocket engine. Janos also attempted to receive signals from Kicksat using a large radio dish.

"I don't think, the small fires on the ground level, help anything for the satellites, it was only a funny idea."

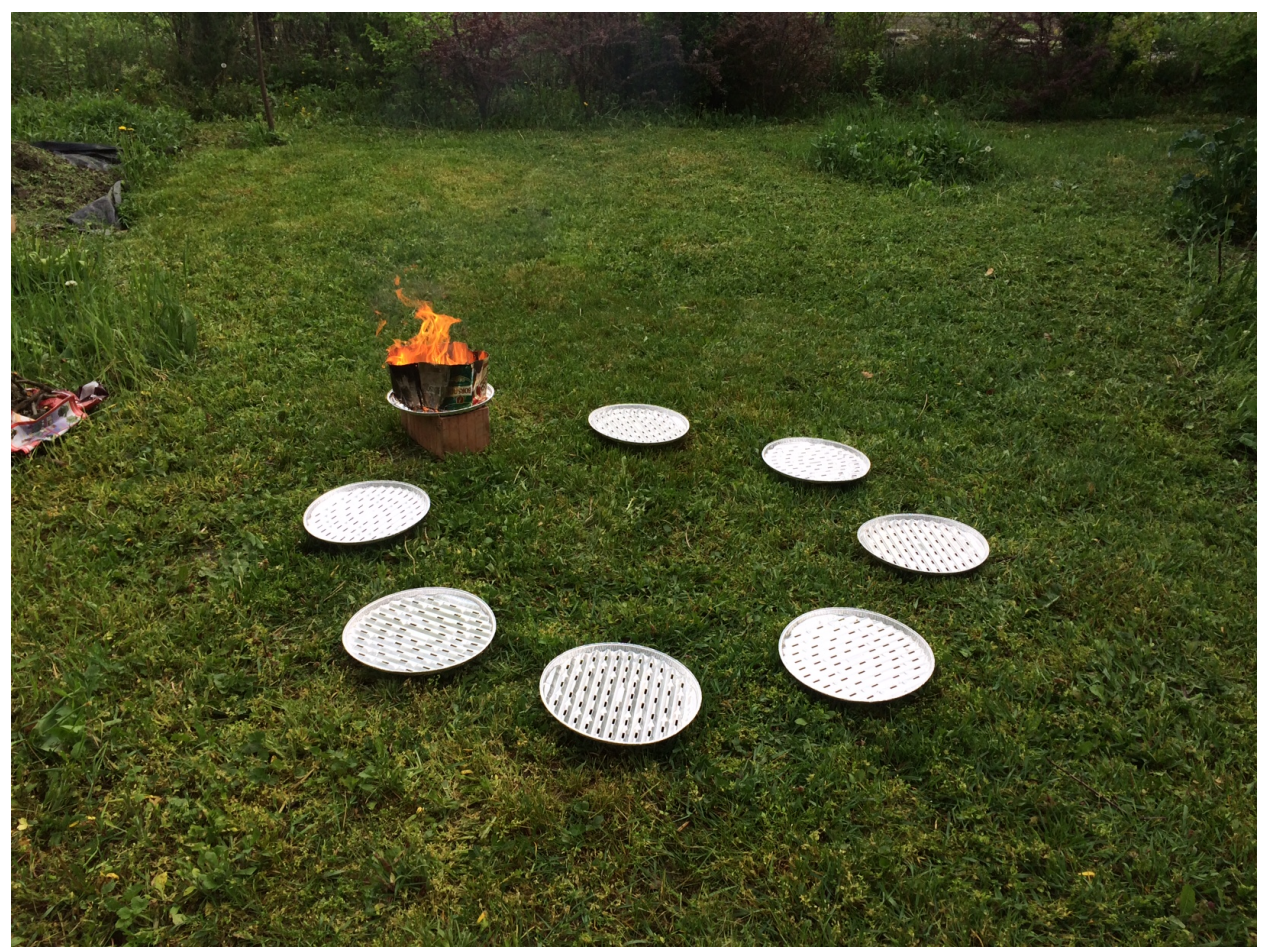

Figure 29: Receive and Recycle, Hungary

Note. By Janos Tolgyesi. With permission.

g) Meet to Delete: Whanganui

May 4, 2014 16:00 NZST. 247 Roberts Avenue, Whangnaui, 4500, New Zealand

The Whanganui event took place on the top of a hill overlooking the town, with a gazebo with an orbital projection and a large bonfire nearby. Participants threw large quantities of documents into the fire, with one person burning boxes of legal case files from an unhappy divorce. One of the few photos of the evening is partially corrupted. 


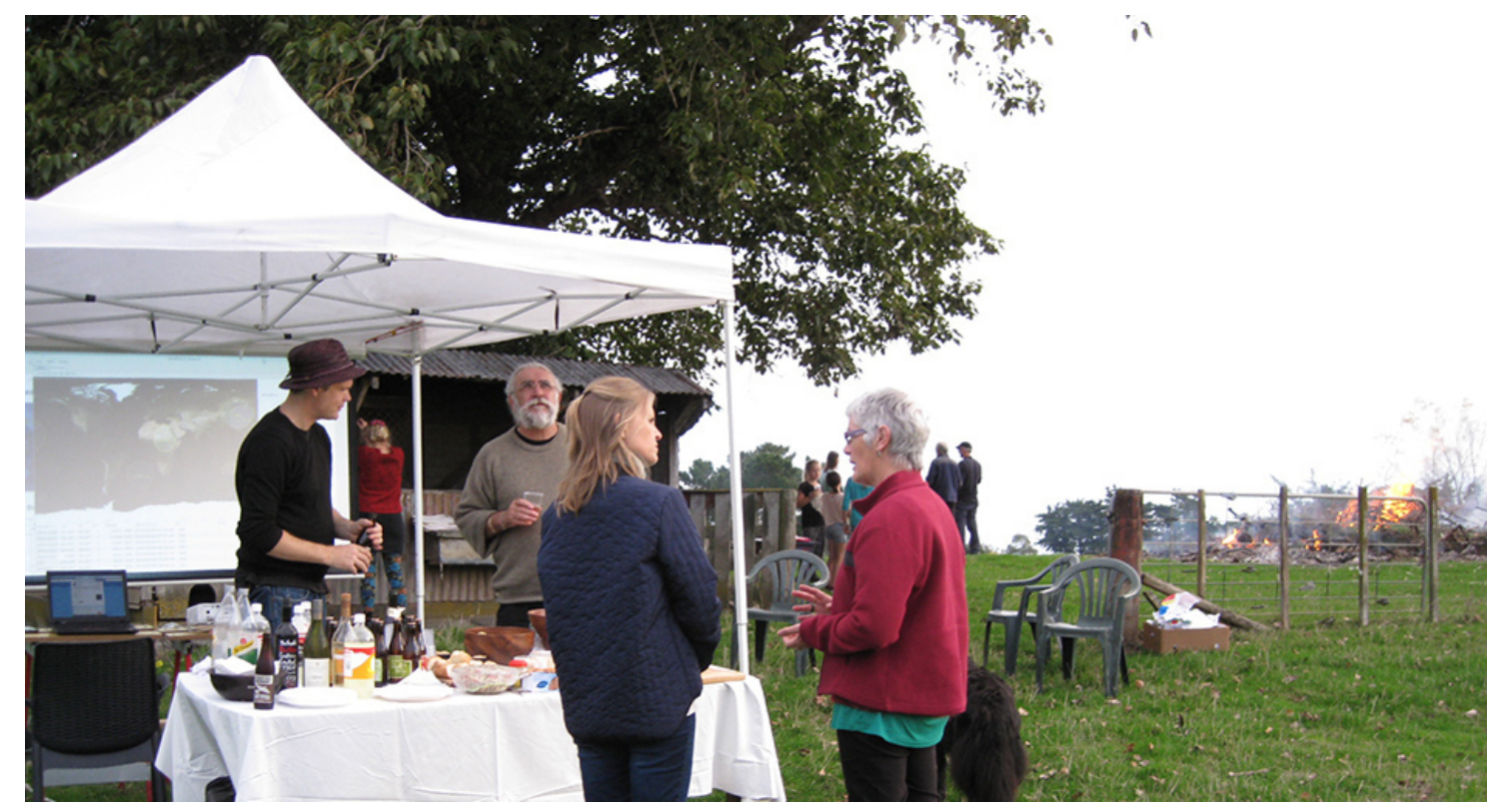

Figure 30: Meet to Delete Whanganui

Note. An open sided tent housed a screen with live satellite position and telemetry data projected onto it. By Brit Bunkley. With permission.

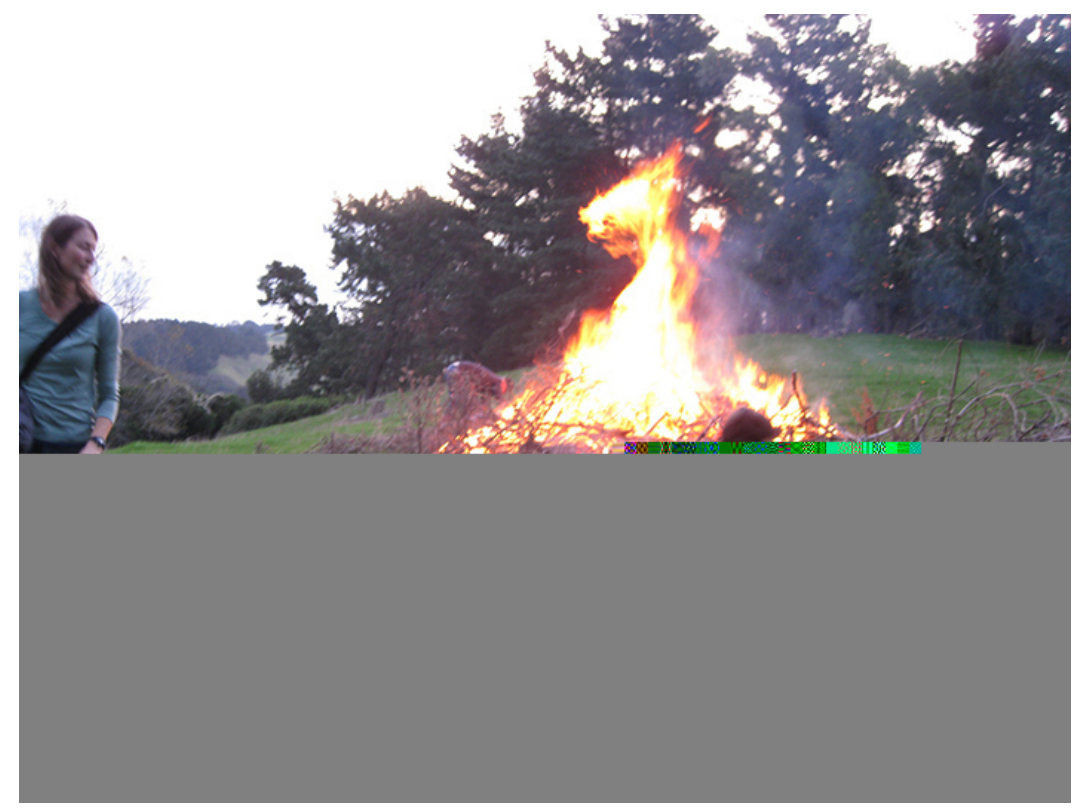

Figure 31: Meet to Delete Whanganui bonfire

Note. A bonfire burns documents. By Julian Priest.

h) Meet to Delete: Ptarmigan

May 4, 2014 16:00 CEST. Kalarand, Kalamaja, Tallinn, Estonia

Media arts group Ptarmigan organised a Meet to Delete bonfire event as part of 
their Strange Meetings Club event series. The bonfire took place on Kalamaja Beach near Tallinn.

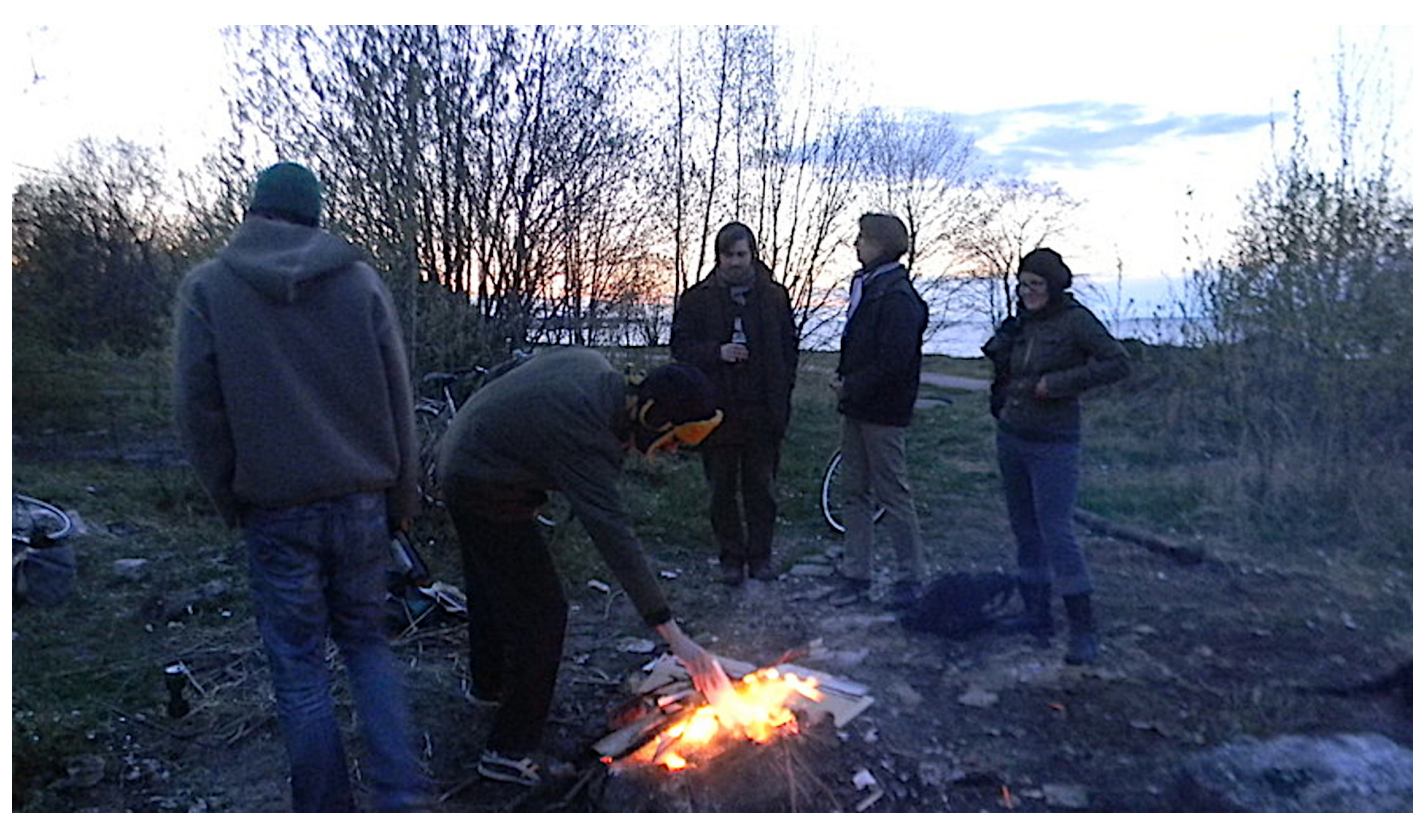

Figure 32: Meet to Delete Ptarmigan

Note. Photos of the Meet to Delete bonfire event. By Ptarmigan (2014). Public Domain.

i) Meet to Delete: Helsinki

May 5, 2014 20:00 CEST. Kaasutehtaankatu 1/21 (Building 7), Suvilahti 00540 Helsinki, Finland

The Meet to Delete event in Helsinki made attempts to delete more data than they produced and documented the quantities of data deleted during and produced during the event.

Deleted: Erased 40 photos (RAW+jpg) approx $30 \mathrm{mb}$ per photo $=1200 \mathrm{MB}$

Erased data from files: $673.5 \mathrm{MB} 768$ mails at $20 \mathrm{~kb}=15360 \mathrm{~kb}=15 \mathrm{MB}$ 246 list posts $=4 \mathrm{MB} 118$ files $=2000 \mathrm{MB} 46$ comics $=184 \mathrm{MB}[$ files $]=920$ $\mathrm{MB}$

Produced.. Taken 26 photos (RAW+jpg) 26x30 = 780MB 1 photo documentation for blog post $. j p g=3 \mathrm{MB}$ Blog post would be maybe $0.02 \mathrm{MB}$ 


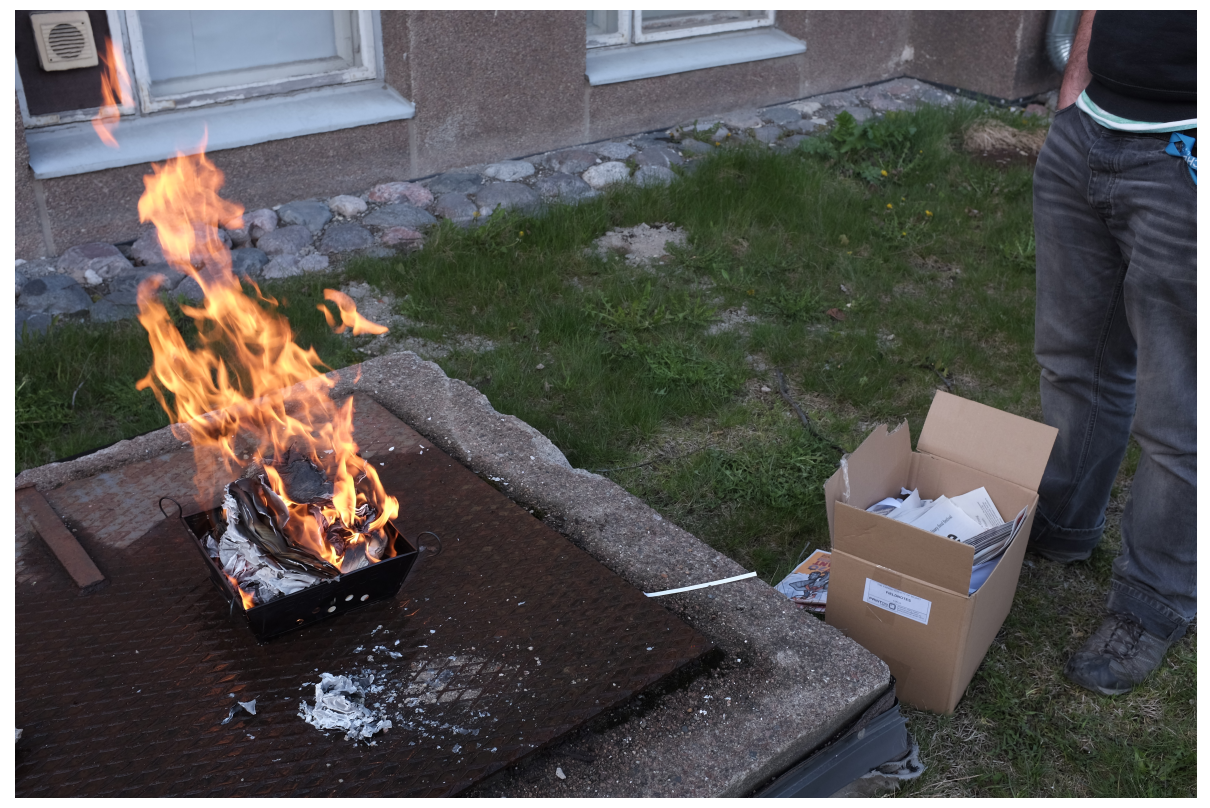

Figure 33: Meet to Delete Pixelache

Note. Photos of the Meet to Delete event Pixelache, Helsinki Pixelache (2014). By Andrew Gryf Paterson. With permission.

j) Meet to Delete: Prescott

May 7, 2014 14:00 MST. 1610 Satellite Court, Prescott, Arizona, USA

Artist John Hopkins visited his uncle and helped him go through his papers to shred documents that he no longer needed. The only documentation was an email and an envelope of paper shreds.

We started with a $30 \mathrm{~cm}$ high stack of documents - bank, credit card, and investment statements, medical records, and other state secrets - from my still-living 99-year-old uncle, who sits nearby uttering a continuous reactionary political commentary ... despite my expressed inability to hear what he was saying over the shredder noise. ...

He asks: "What are you shredding?"

I answer: "A bunch of your papers from more than seven years ago that Gladys (his sister-in-law) left in a pile to be shredded. Somebody's gotta do it, somebody has to spend life-time and life-energy to destroy this stuff, you can't just throw all these medical papers in the bin, they'll steal your identity!" 
He replies without speaking: "I don't care about that."

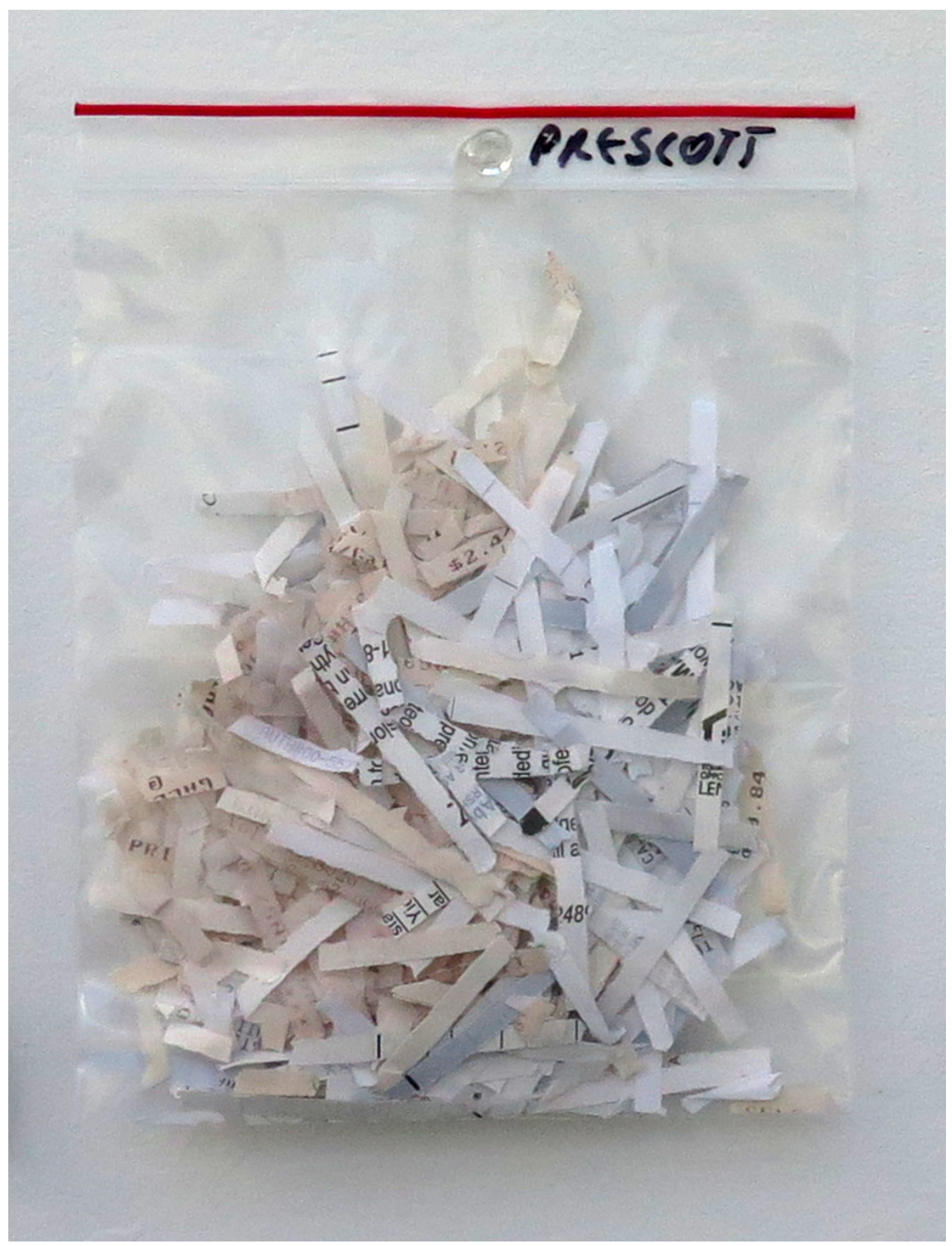

Figure 34: Meet to Delete Arizona

Note. Paper shreds from Meet to Delete: Prescott. These were physically posted as the only documentation of the event and arrived by post. The paper shreds were shown as part of the Engine Room exhibition. By Julian Priest.

k) Meet to Delete: Bogota

May 9, 2014 19:30 COT. Bogota, Colombia

Artist Andres Burbano organised guests to an event in Bogota. The event focused on deleting unwanted data from participants personal devices, laptops and 
phones.

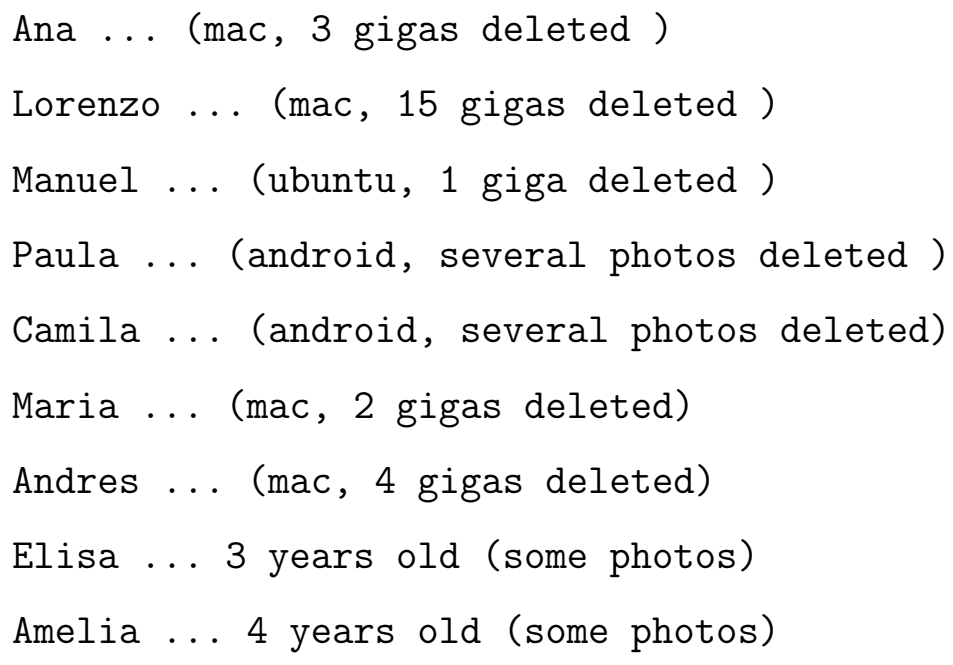

Figure 35: Meet to Delete Bogota Meta Data

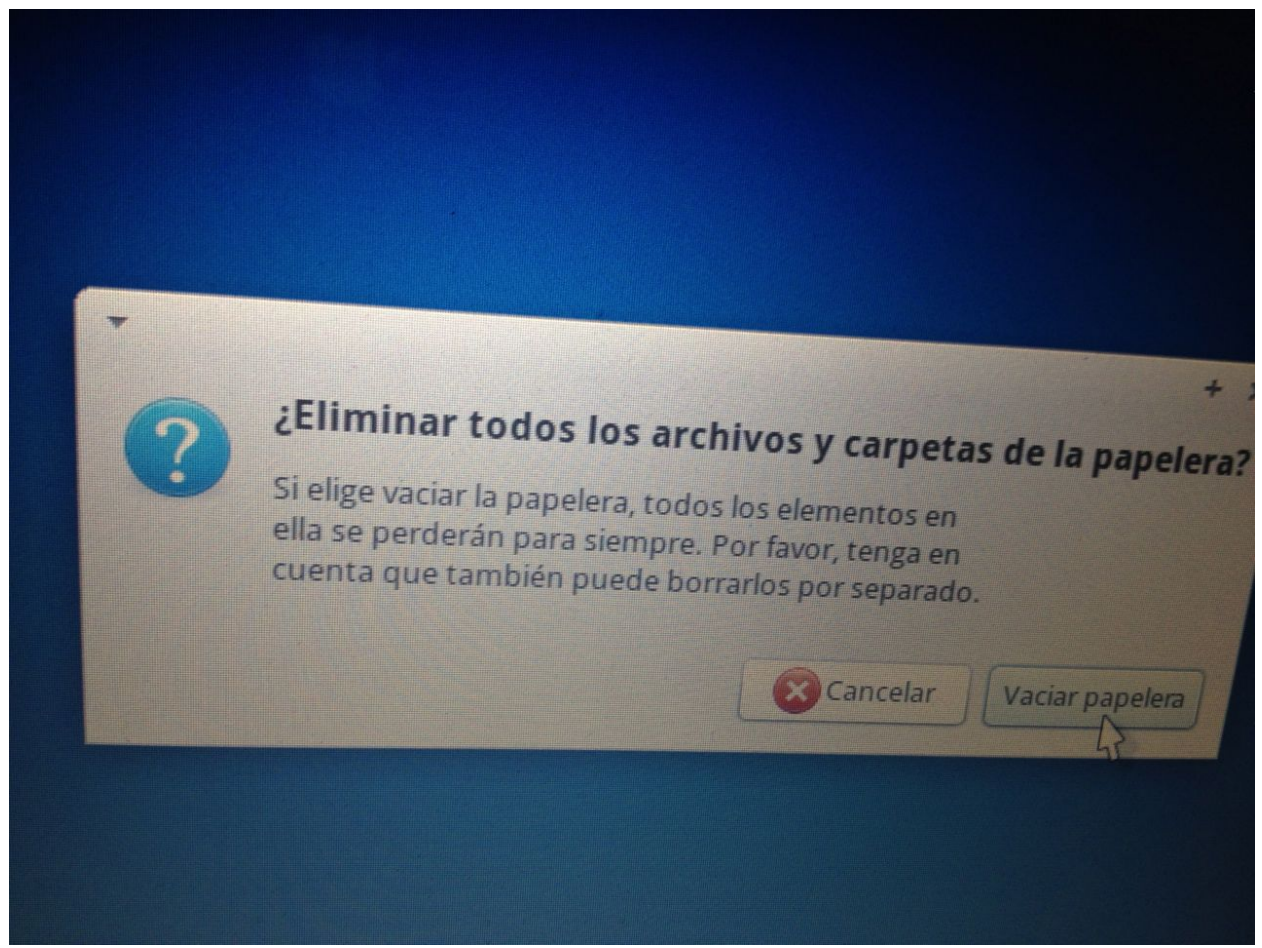

Figure 36: Meet to Delete Bogota

Note. Emptying the waste bin on a computer at the Bogota Meet to Delete. By Andres Burbano. With permission. 


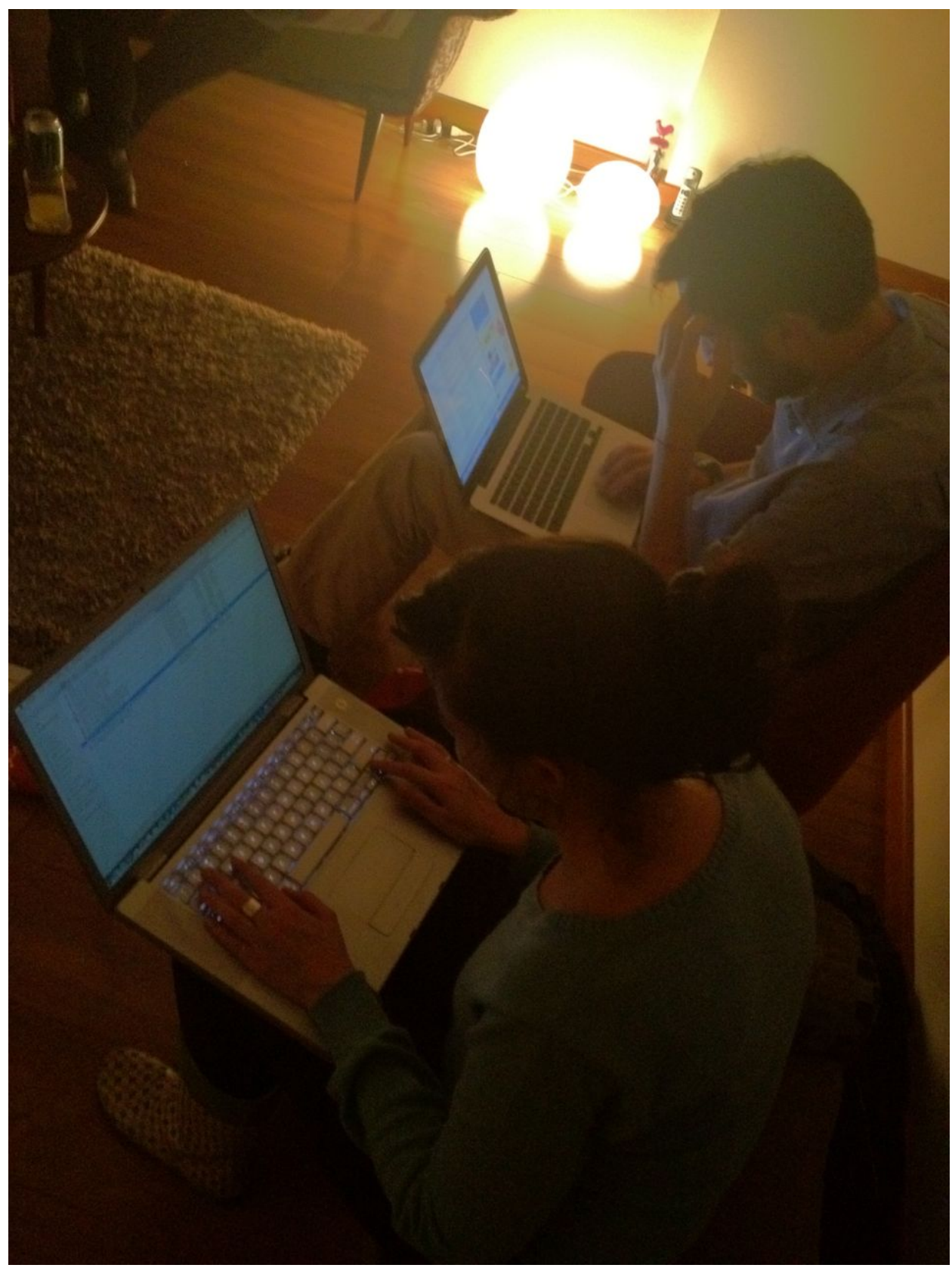

Figure 37: Meet to Delete Bogota

Note. View of the Bogota Meet to Delete workshop. By Andres Burbano. With permission.

l) Re-entry

May 14, 2014 01:30 UTC Upper Atmosphere 

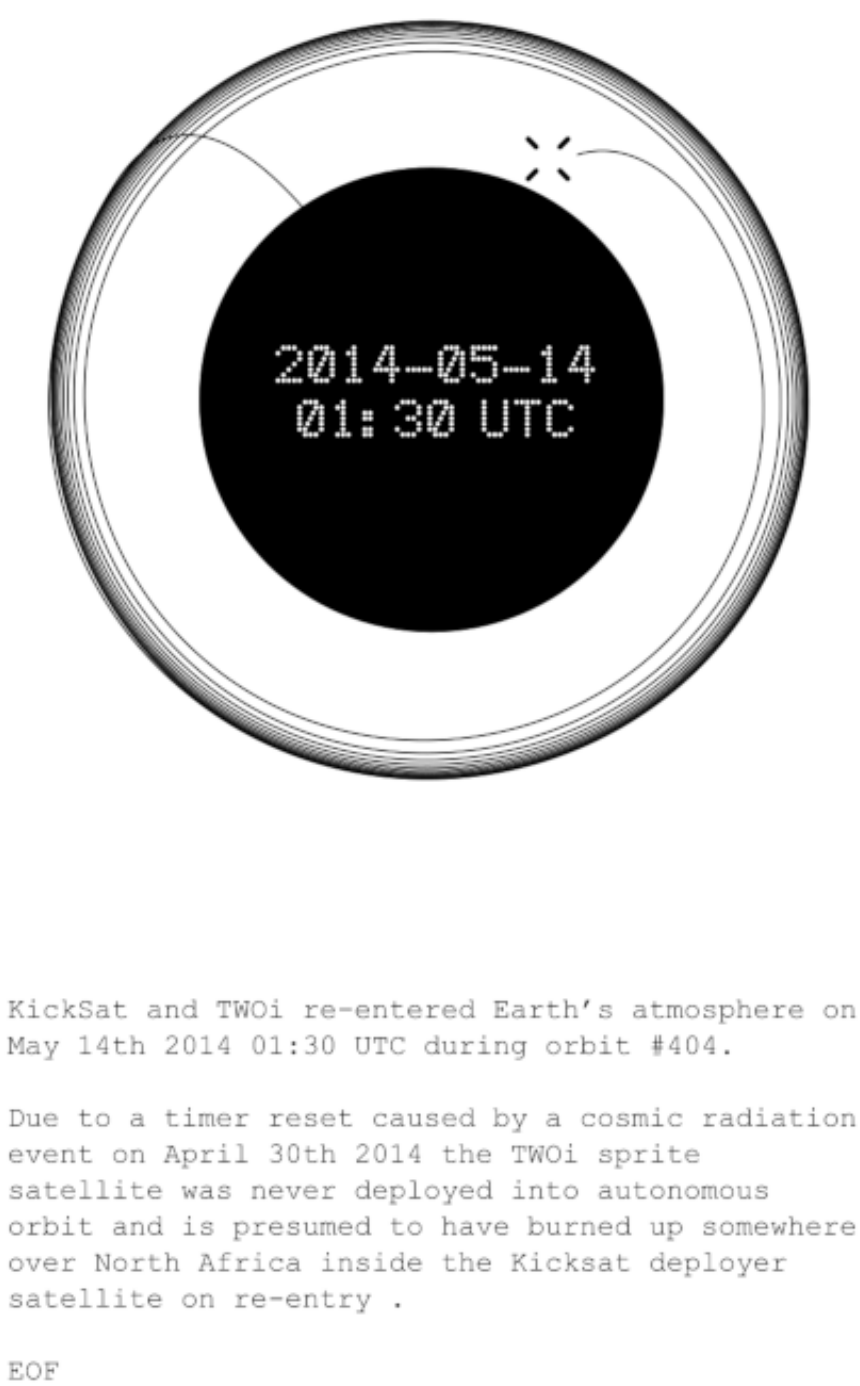

Figure 38: TWOi Re-entry Poster

Note. Online and print poster distributed on the occasion of TWOi re-entering Earth's atmosphere and burning up. EOL refers to the acronym End of Life. EOF refers to the symbol for End of File. By Julian Priest.

4. $2015-2018$

In the period after the first TWOi launch the work was presented at a number of conferences and symposia. In 2015 NASA announced that Kicksat2 was manifested in their 
launch program and preparations were made for a second TWOi project. A new requiem work TWOi Ground Station, based on ephemera from the launch and meet 2 delete events was developed during an artist residency and shown at the Engine Room. Wellington City Council partially funded a new TWOi 2.0 project to coincide with the new launch, and then there was a multi year delay due to regulatory issues before a launch date was set.

a) Ada Symposium 2014

September 12, 2014. Colab, A.U.T Auckland, New Zealand

A talk about the project was given at the Ada Symposium 2014, Mesh Cities in a panel entitled Data Funerals with Audrey Samson chaired by Ruth Irwin.

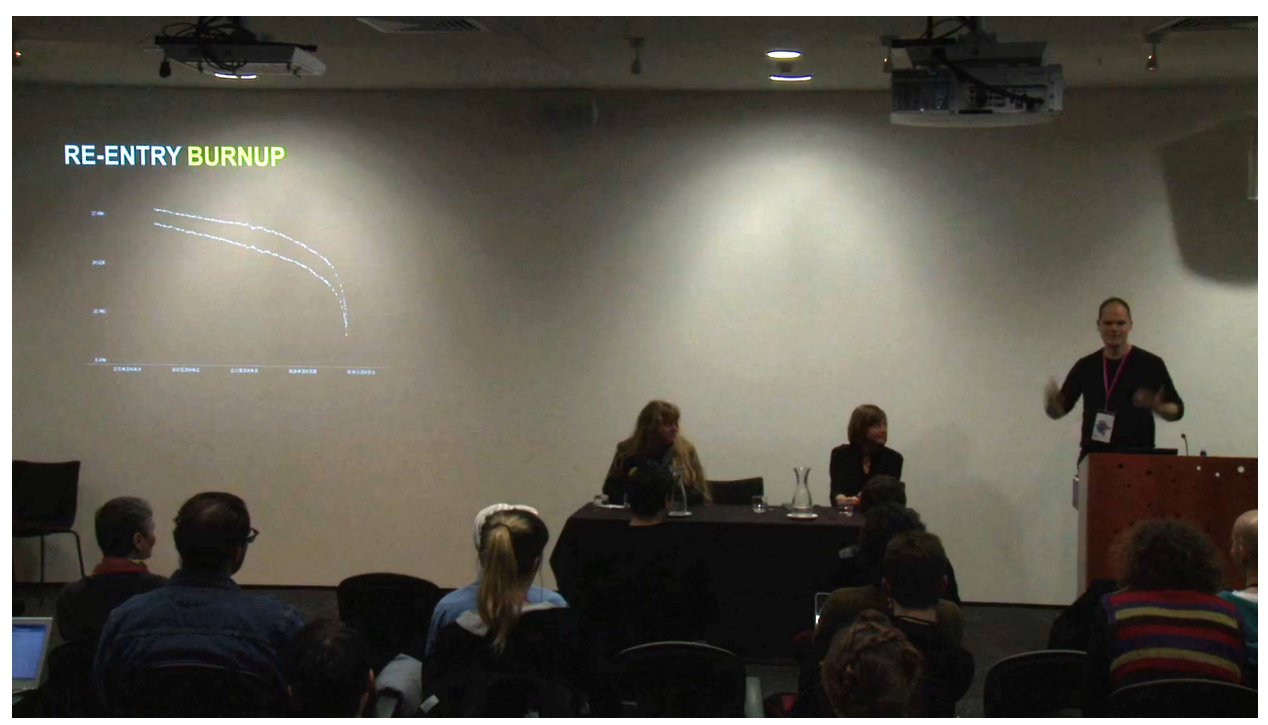

Figure 39: Ada Symposium 2014

Note. Screengrab of Ada symposium proceedings. By Ada.net.nz (2014). Creative Commons Attribution-NonCommercial-NoDerivs 2.5.

b) Arduino Day 2015

March 28, 2015. School of Design, Victoria University of Wellington, New Zealand A talk about the project was given at the Arduino Day to an audience mainly of Arduino developers, makers and hobbyists.

c) Energies and Arts 2015

August 15, 2015. UNSW Art \& Design, Sydney, Australia 
A talk about the project was given in the artist's track of an interdisciplinary academic conference about energies and arts at UNSW and the Museum of Contemporary Art, Australia (Artdesign.unsw.edu.au, 2015).

\section{d) TWOi Ground Station}

November 06-22, 2015. The Engine Room, Massey University, Wellington, NZ

In 2015 during a 3 month artist residency at Massey University a gallery exhibition and installation were developed. The exhibition was based on the 2014 TWOi flight and was framed as a requiem work for the TWOi satellite. A satellite tracking robot with an antenna was programmed to replay orbital data from the 2014 flight. It followed the satellite's historical track across the sky, becoming active for 15 minutes when the satellite would have been overhead. A series of screens mounted on plinths showed the launch of the rocket, NASA footage of the orbit, the historical track of the satellite and a null output from the radio receiver as sound and image. Other ephemera were arranged on the walls including; paper shreds and ash from the meet to delete events, a poster detailing the satellites demise and a scale showing a negative weight of a Kicksat satellite. The exhibition

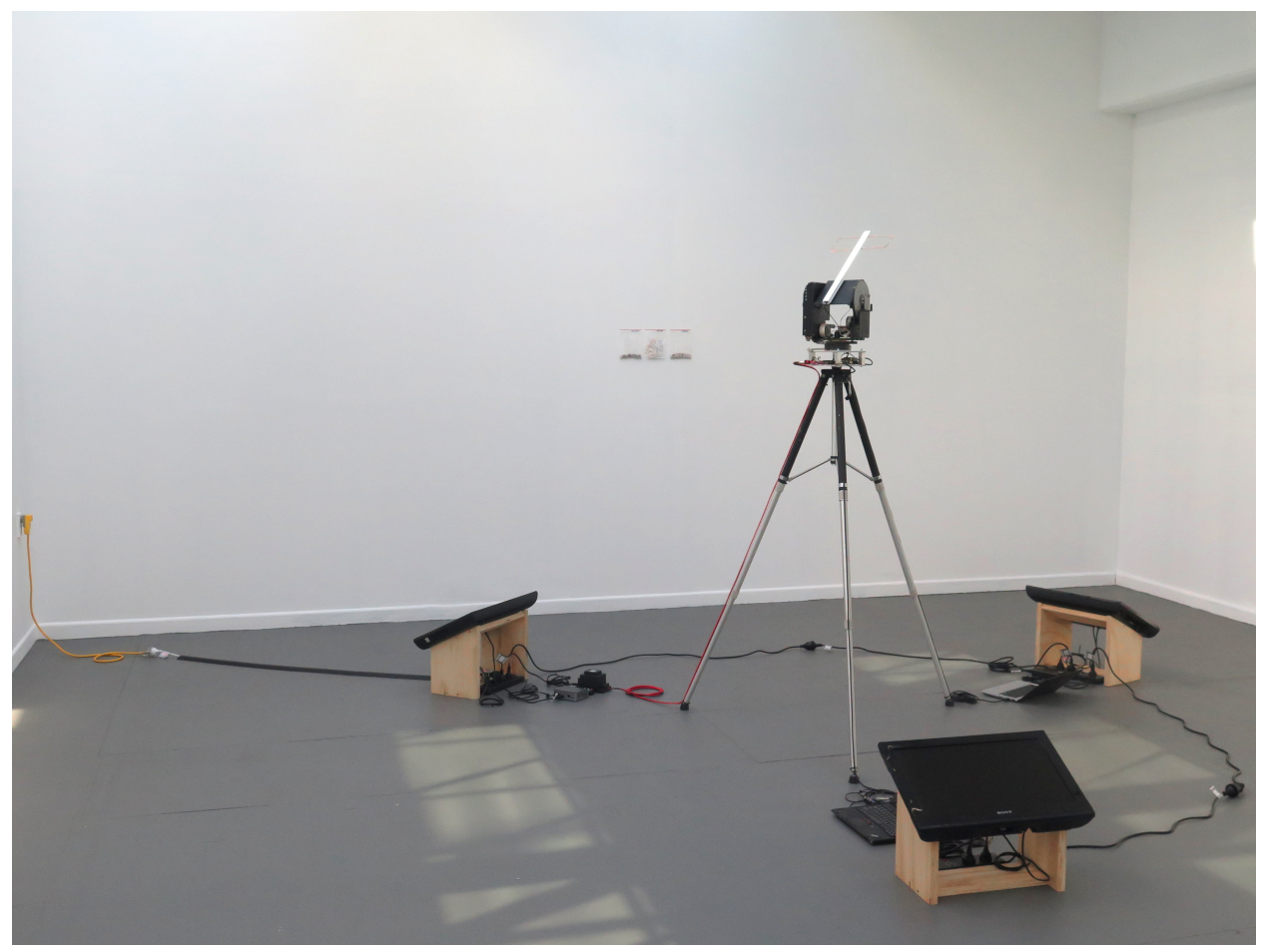

Figure 40: The Weight of Information Ground Station Layout

Note. 3 screens on plinths arranged around a central tracking robot. Ephemera is 
mounted on the walls. By Julian Priest.

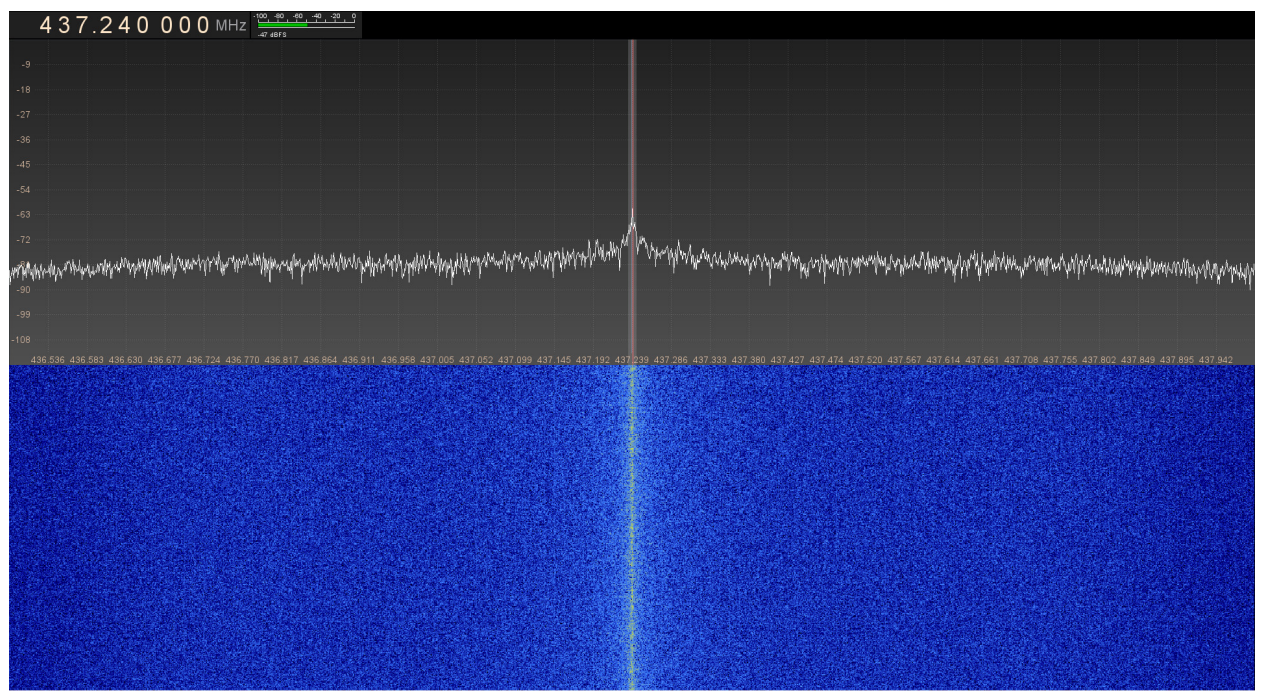

Figure 41: The Weight of Information Ground Station Radio

Note. Screen grab from the radio receiver showing a null output with no transmission received. By Julian Priest.

5. $2018-2019$

a) TWOi 2.0 Thomas King Observatory Residency

October 30 - December 12, 2017. Thomas King Observatory, Wellington, NZ

A second Kicksat launch was announced in 2015 and TWOi manifested to fly as part of the program. A re-run of the project now named TWOi 2.0 was developed and partially funded by Wellington City Council Public Art Fund. In 2017 an artist residency was secured at Wellington Museum's Space Place in which the disused Thomas King Observatory was offered as a building to house the new TWOi 2.0 project. After an initial series of test satellite observations at the TKO in late 2017 established the viability of the space, and renovation of the building in 2018 , the residency took place over a year between March 2018 and April 2019. 


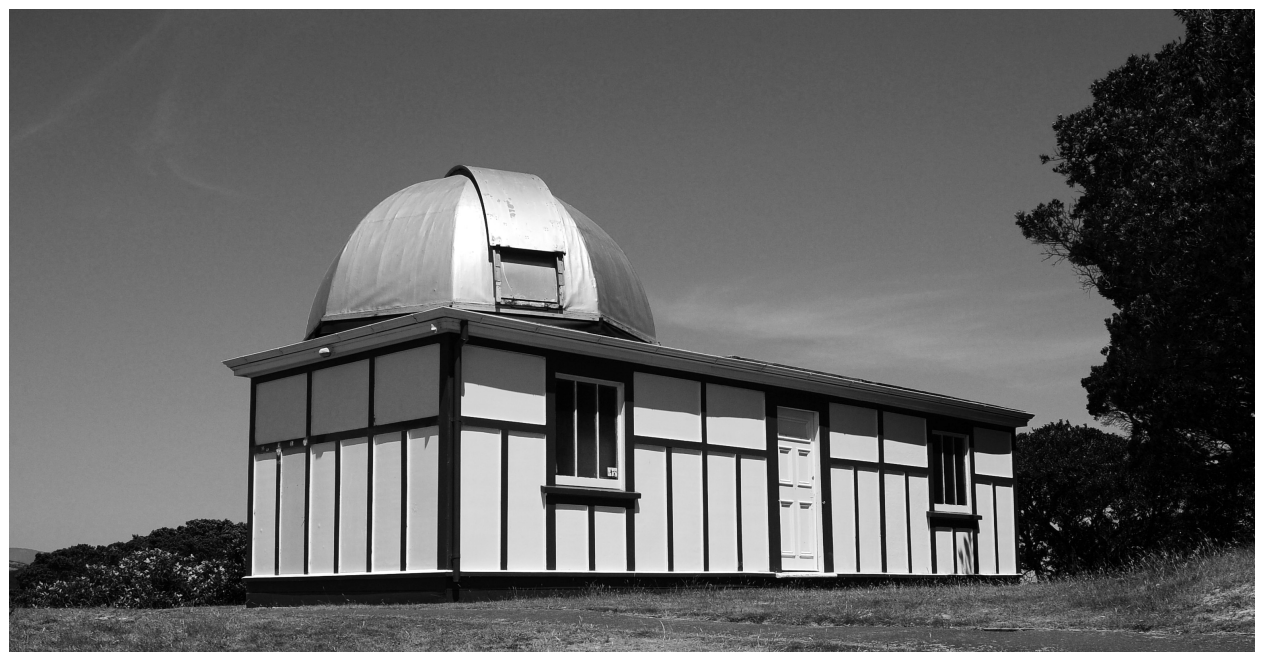

Figure 42: Thomas King Observatory

Note. The Thomas King Observatory. By Julian Priest.

b) TWOi 2.0 Opening

November 15, 2018. The Planetarium, Space Place, Carter Observatory, Wellington, $N Z$

A public talk to launch the second iteration of the project TWOi 2.0 was given with images projected inside the planetarium dome on the night of the launch. The public talk was scheduled for the night of the planned launch and a launch vigil started directly afterwards.

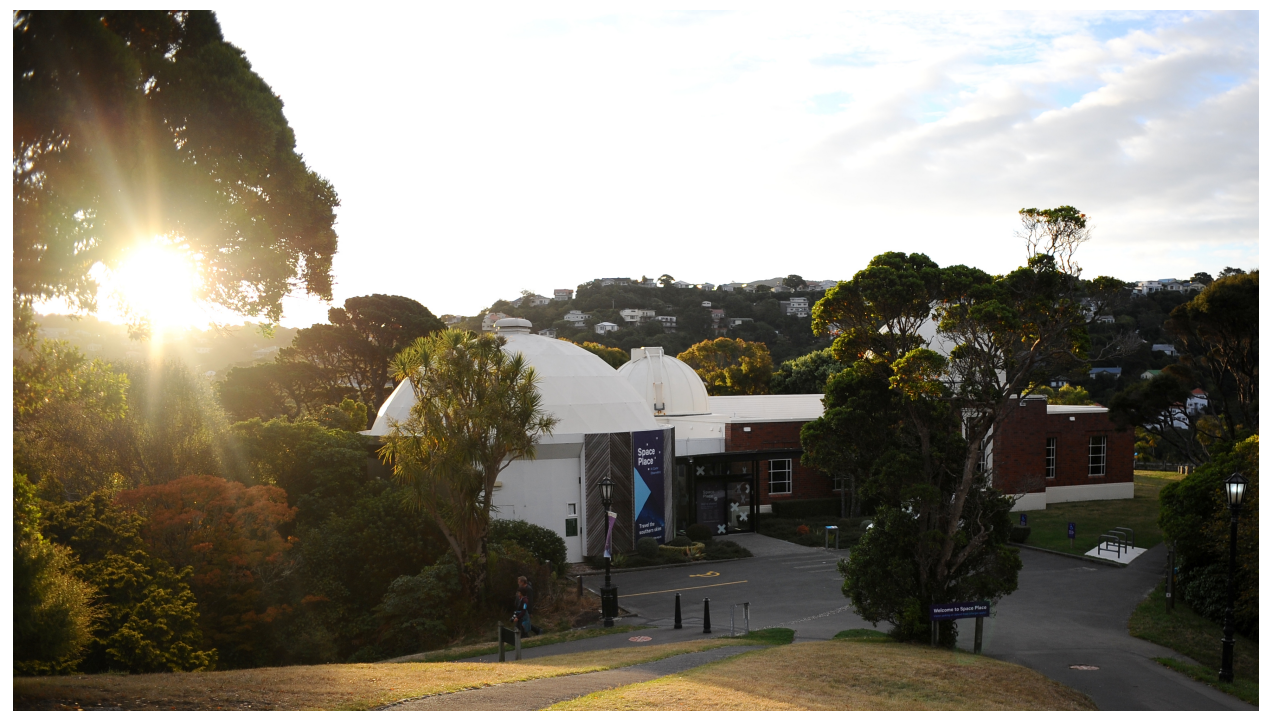

Figure 43: Space Place Carter Observatory

Note. Space Place is housed in the Carter Observatory in the Wellington Botanic 
Gardens. By Observatory (2018). Creative Commons Attribution Share Alike License.

c) TWOi 2.0 Launch Vigil

November 15 - 17, 2018. Thomas King Observatory, Wellington, NZ

The Thomas King Observatory was open to the public for a launch vigil during which live streams of the launch were shown. Kicksat2 was launched from Wallops Flight Facility, Virginia, USA on board ISS resupply mission NG-10 in a Cygnus space craft. After two evenings of delays the NG-10 rocket was successfully launched at 21:16 EDT, November 17 2018. The NG-10 mission was sent to the ISS and docked there for 3 months while supplies were taken from the Cygnus, which was then repacked with waste. During this period Kicksat2 remained inactive in its Nanoracks deployer module. During the launch vigil TWOi was featured on Radio New Zealand Freeman (2019).

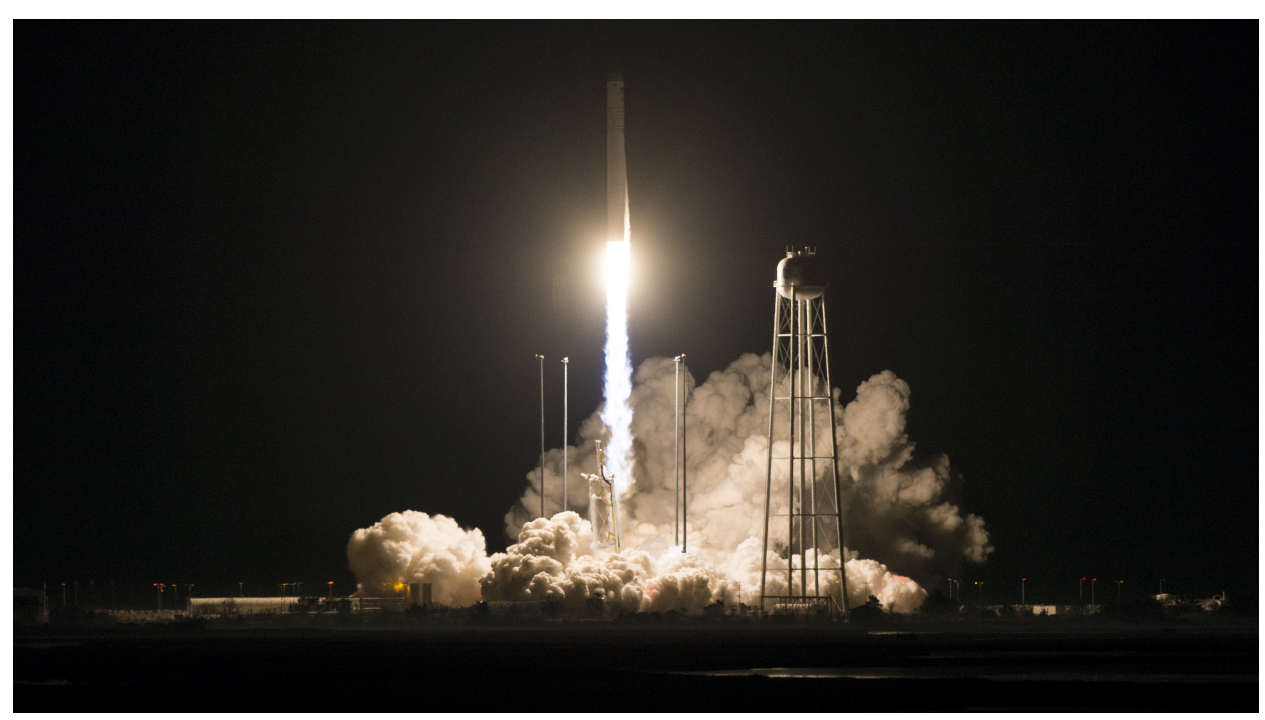

Figure 44: NG-10 Launch

Note. Launch of NG-10 from Wallops Flight Facility, Virginia, USA. By J. K. NASA (2018). Public Domain. 


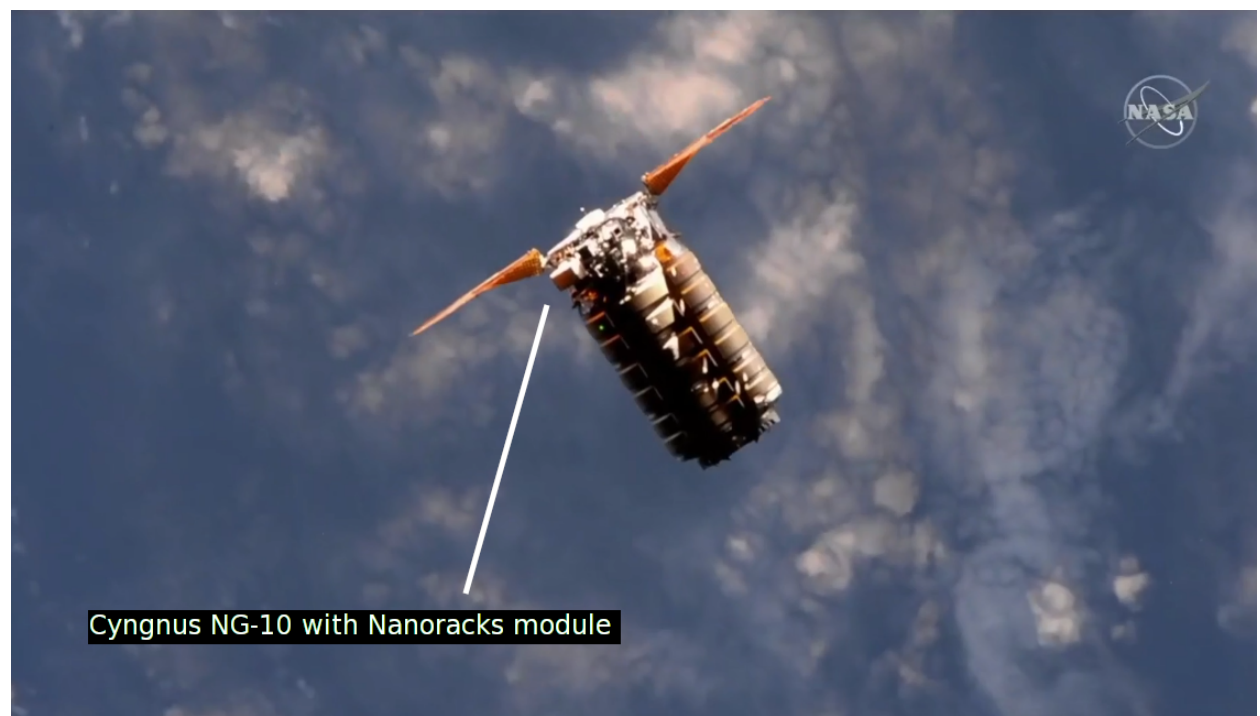

Figure 45: NG-10 Cygnus Flight

Note. Labelled frame grab of a video of the NG-10 Cygnus flight to the ISS showing position of the Nanoracks deployer module that contained Kicksat2 and TWOi. By NASA (2018). Public Domain.

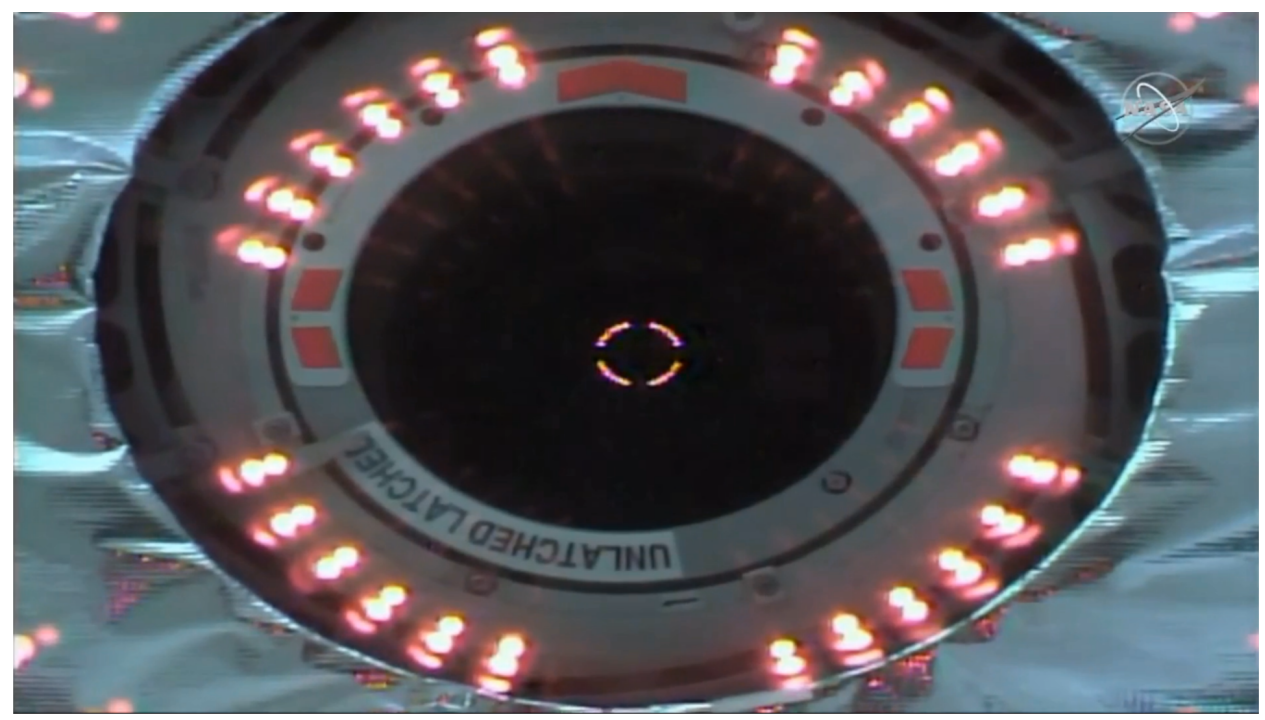

Figure 46: NG-10 ISS Docking

Note. Frame grab of a video of the NG-10 Cygnus flight showing docking with the ISS. By NASA (2018). Public Domain.

d) Meet 2 Delete

February 15 - 24, 2019 11:00 - 18:00 NZDT Thomas King Observatory, Wellington, $N Z$ 
The NG-10 spacecraft carrying Kicksat2 and TWOi 2.0 unberthed from the ISS on February 8, 2019, and the Kicksat deployer satellite was released on on February 13, 2019. The Thomas King Observatory was open to the public daily until TWOi re-entered the atmosphere on April 4, 2019.

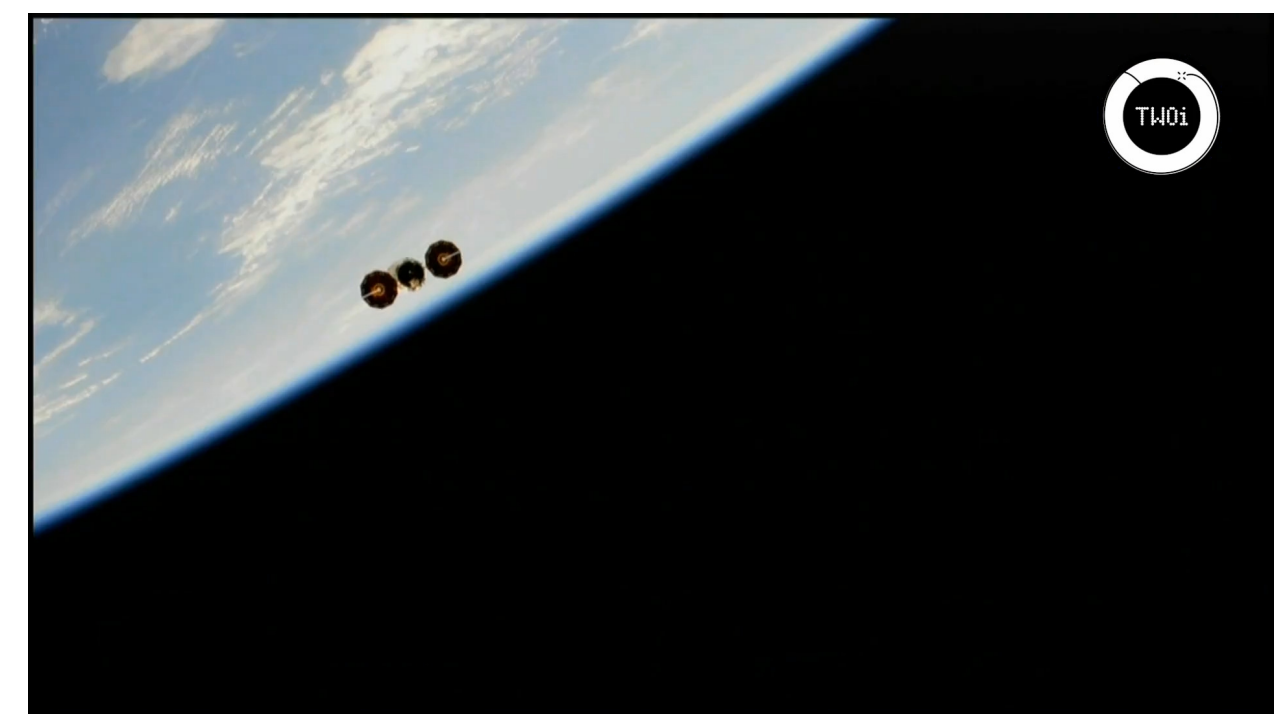

Figure 47: TWOi 2.0 Video

Note. Frame grab from video displayed in the TKO showing the NG-10 leaving the ISS with Kicksat2 and TWOi 2.0 on board. The video was a compilation of scenes from the NG-10 NASA flight videos composited as the exhibition developed. Composite by Julian Priest (2019). Public Domain.

The exhibition had two rooms. The first contained the weighing scales and development satellite from the Engine Room exhibition as well as 2 projections and a workstation with large screen. One projection showed video of the launch, unberthing and 3d simulation of the Kicksat deployment, and the second showed the real time position of Kicksat with data updated from NORAD. The workstation showed radio data from from the antenna and other telemetry as needed. The media changed over the course of the mission. 


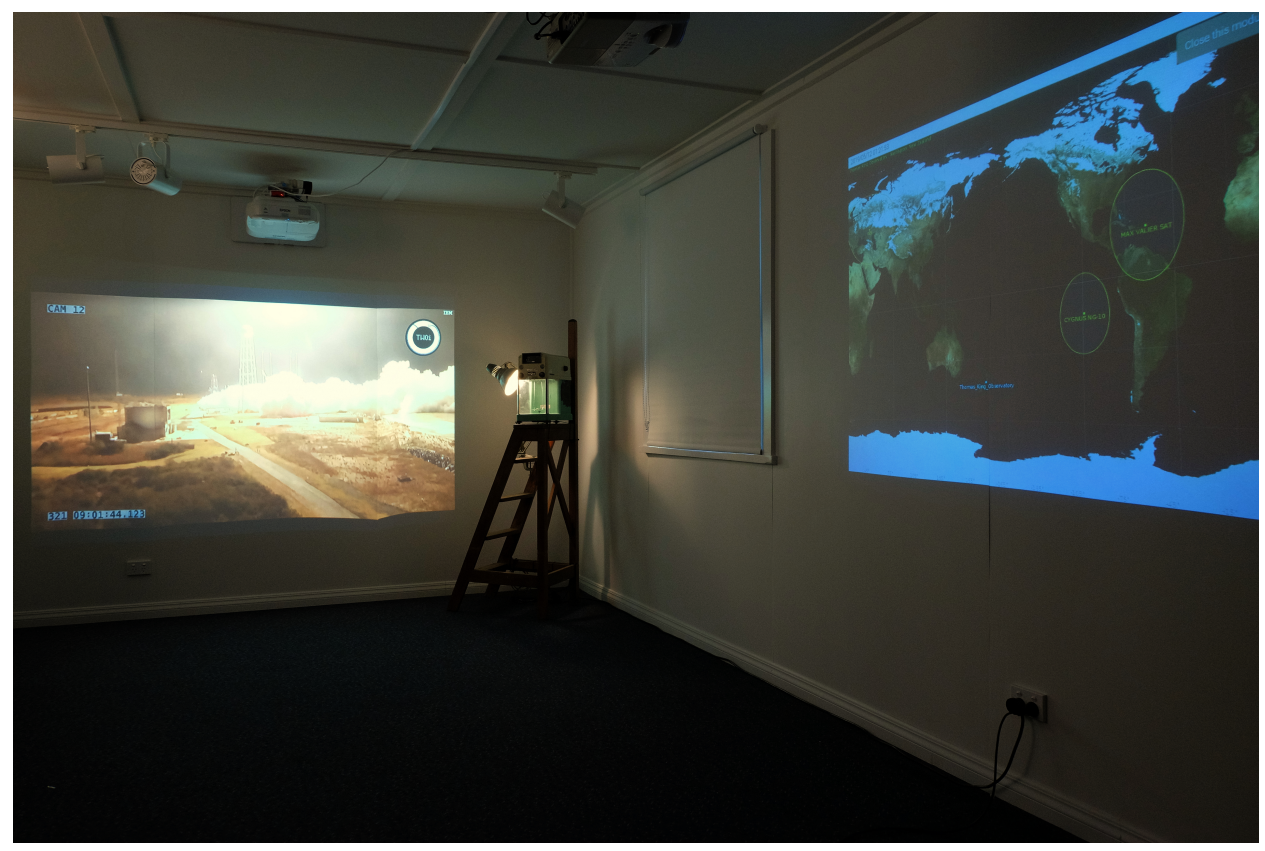

Figure 48: TWOi 2.0 Mission Control Room

Note. By Julian Priest.

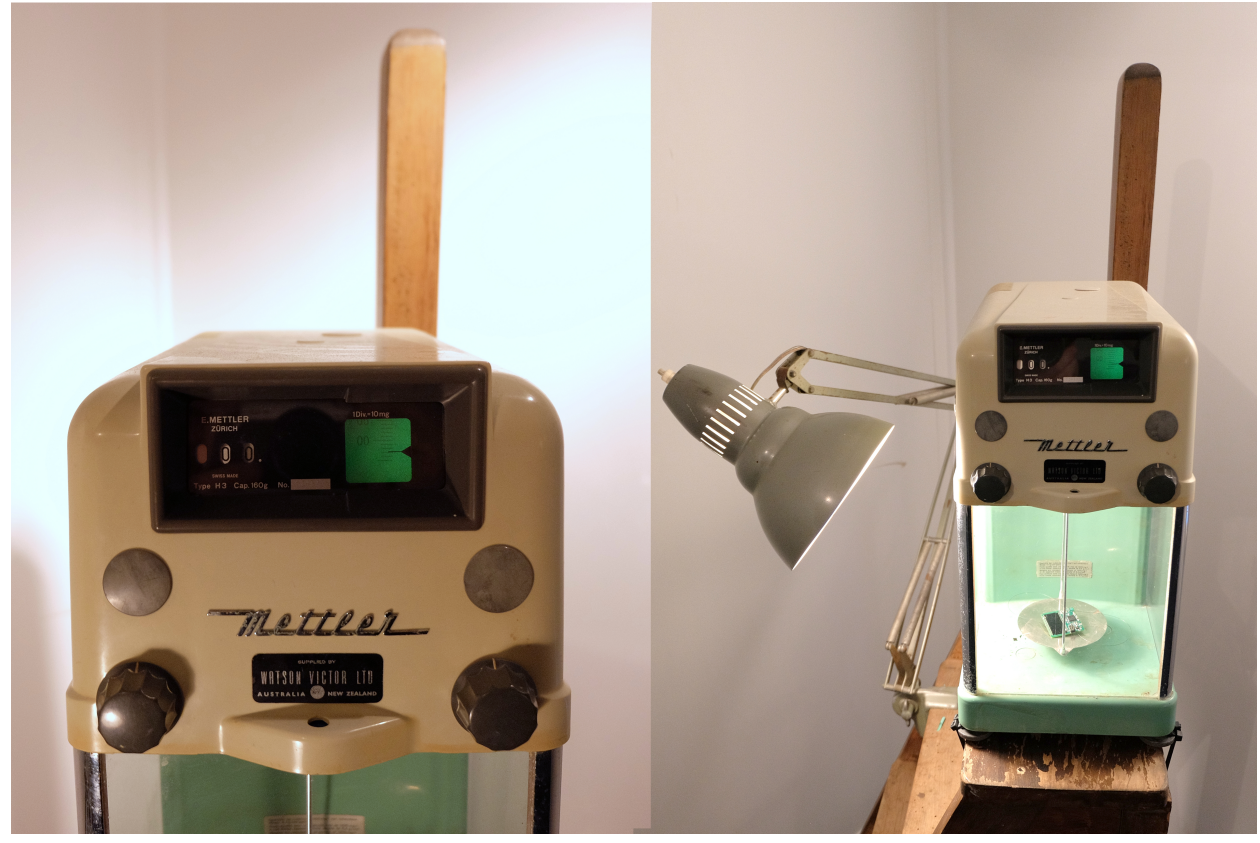

Figure 49: TWOi 2.0 Satellite Balance

Note. Modified precision balance with satellite, showing negative weight. By Julian Priest. 


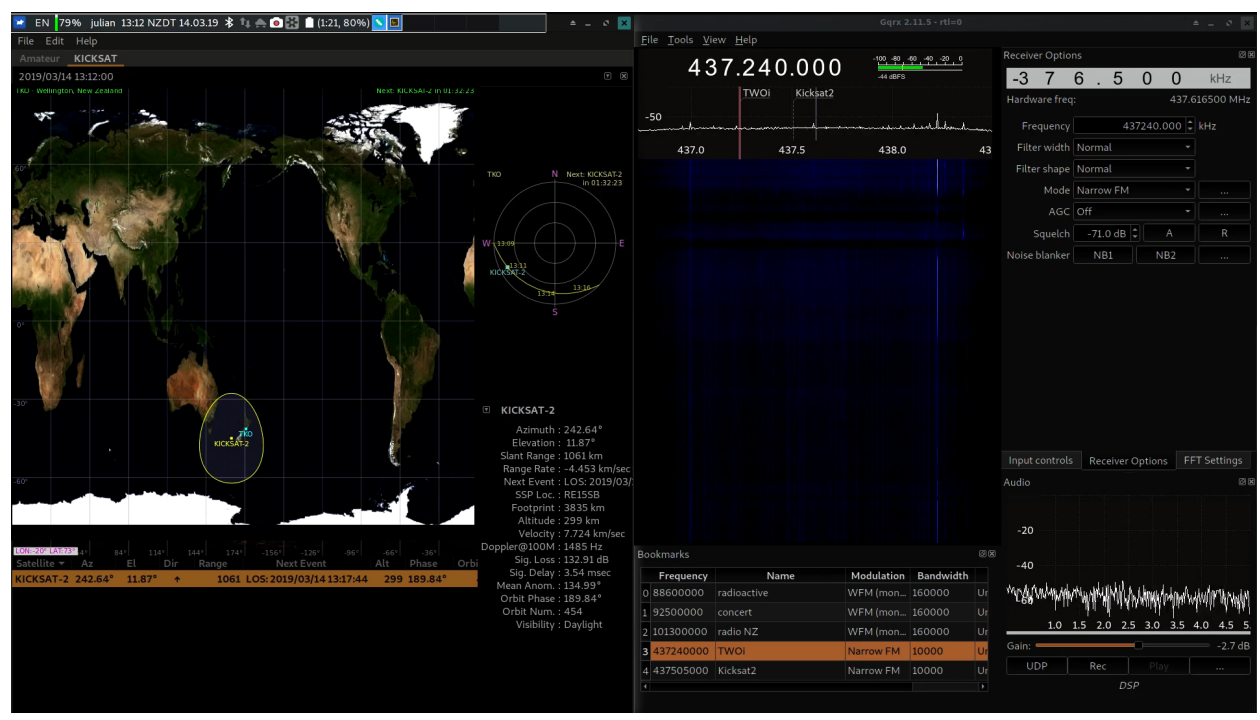

Figure 50: TWOi 2.0 Telemetry

Note. Screenshot from telemetry screen. By Julian Priest.

The second room was the historic observatory dome with a wooden roof, which housed a satellite tracking robot on a plinth which would follow the TWOi satellite as it made passes over the observatory. The wooden roof allowed radio signal to pass through it but did attenuate slightly. The rotator was also used outdoors for some satellite passes. During the orbital period there were typically 2-3 passes over head each 24 hours.

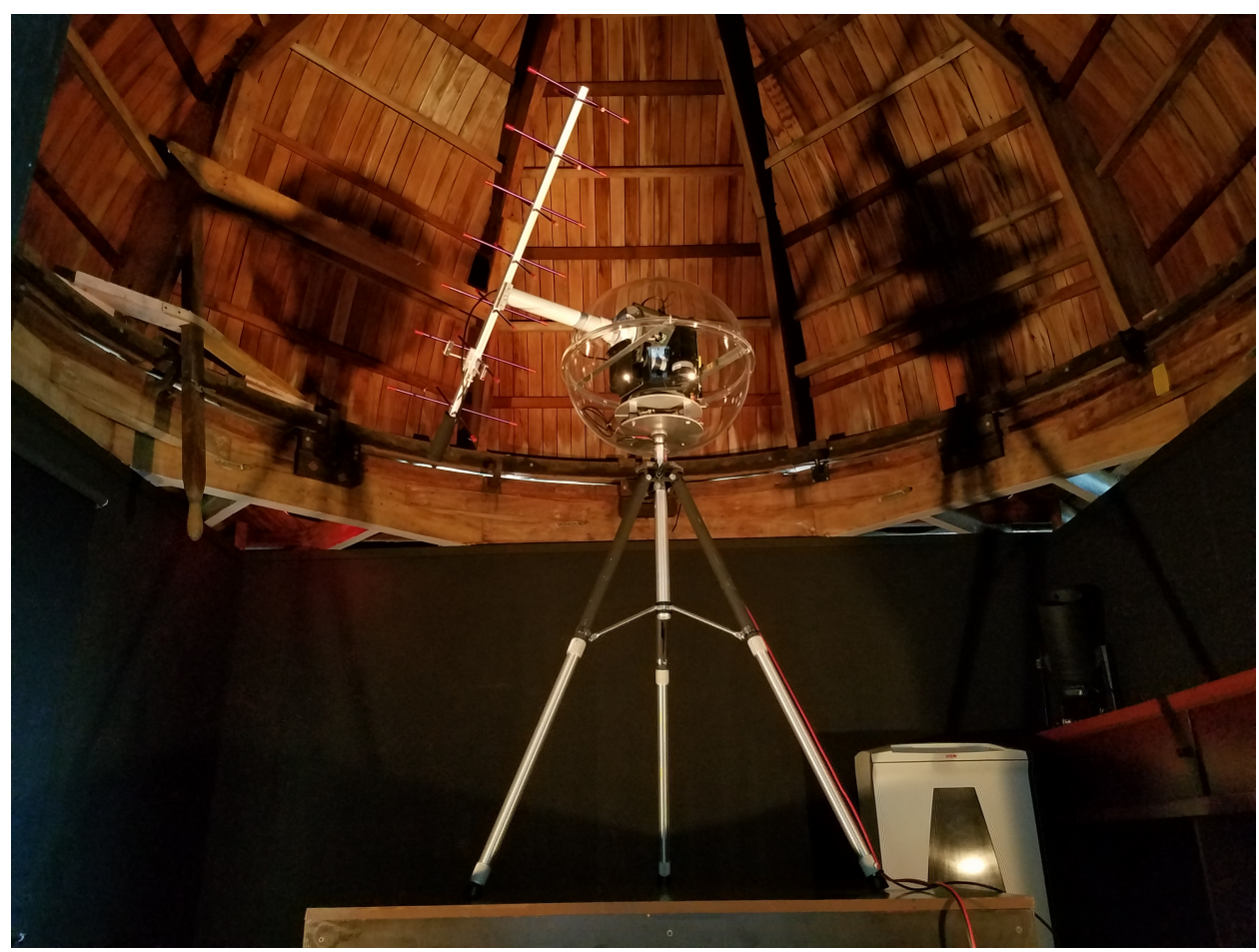


Note. Antenna Rotator robot mounted in TKO dome room. By Julian Priest.

In the corner of the dome room was a cross cut paper shredder. Participants were invited to bring information to delete, or use pen and paper to write something that they wished to delete. They were then invited to go into the observatory and shred the paper, before depositing it into a confetti cannon. The cannon was then activated and the paper shreds shot into to observatory dome lit by a strobe light. Over the course of the exhibition 438 visitors participated and the dome filled with paper shreds of deleted information. 438 people participated in the Meet 2 Delete events over the course of the orbit.

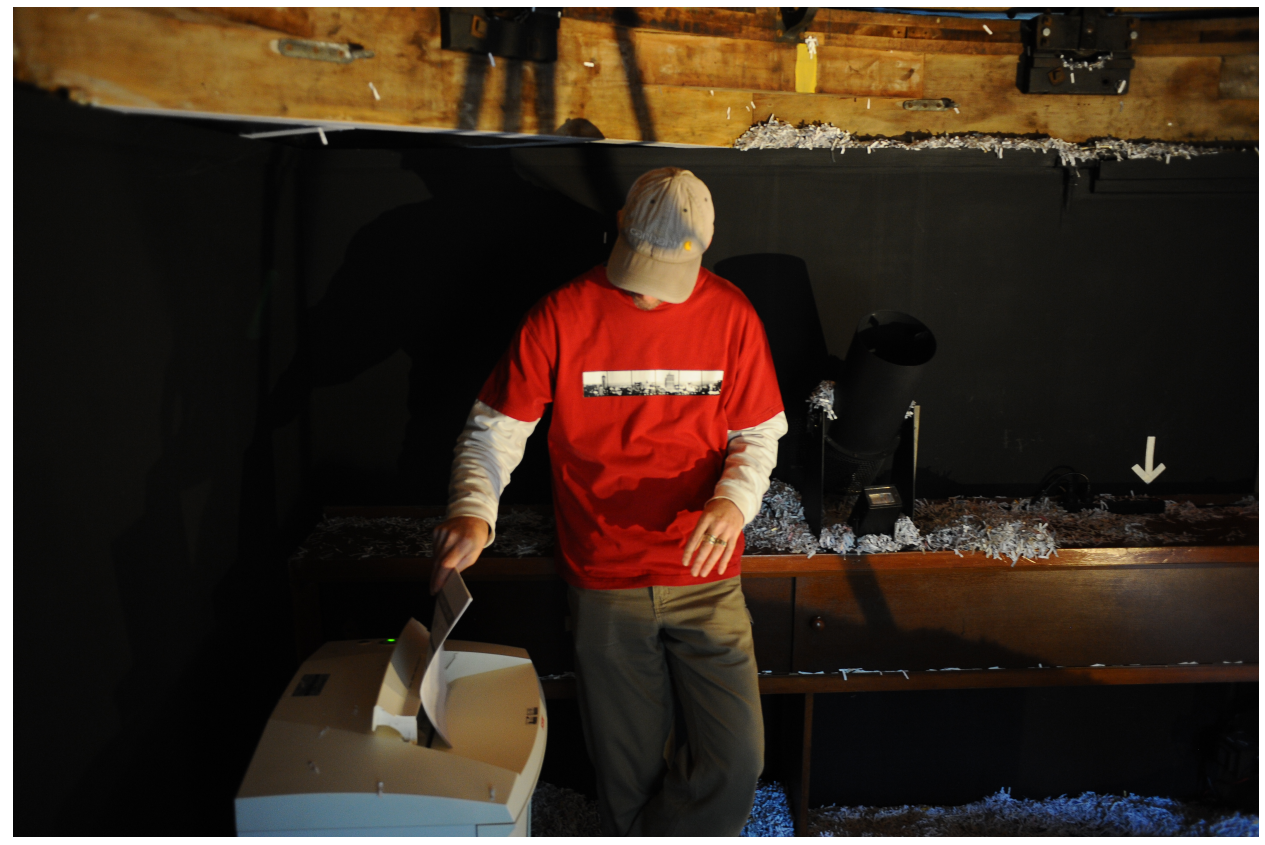

Figure 52: Meet 2 Delete Paper Shredding

Note. Exhibition visitor shredding boxes of old accounts files. By Julian Priest. 


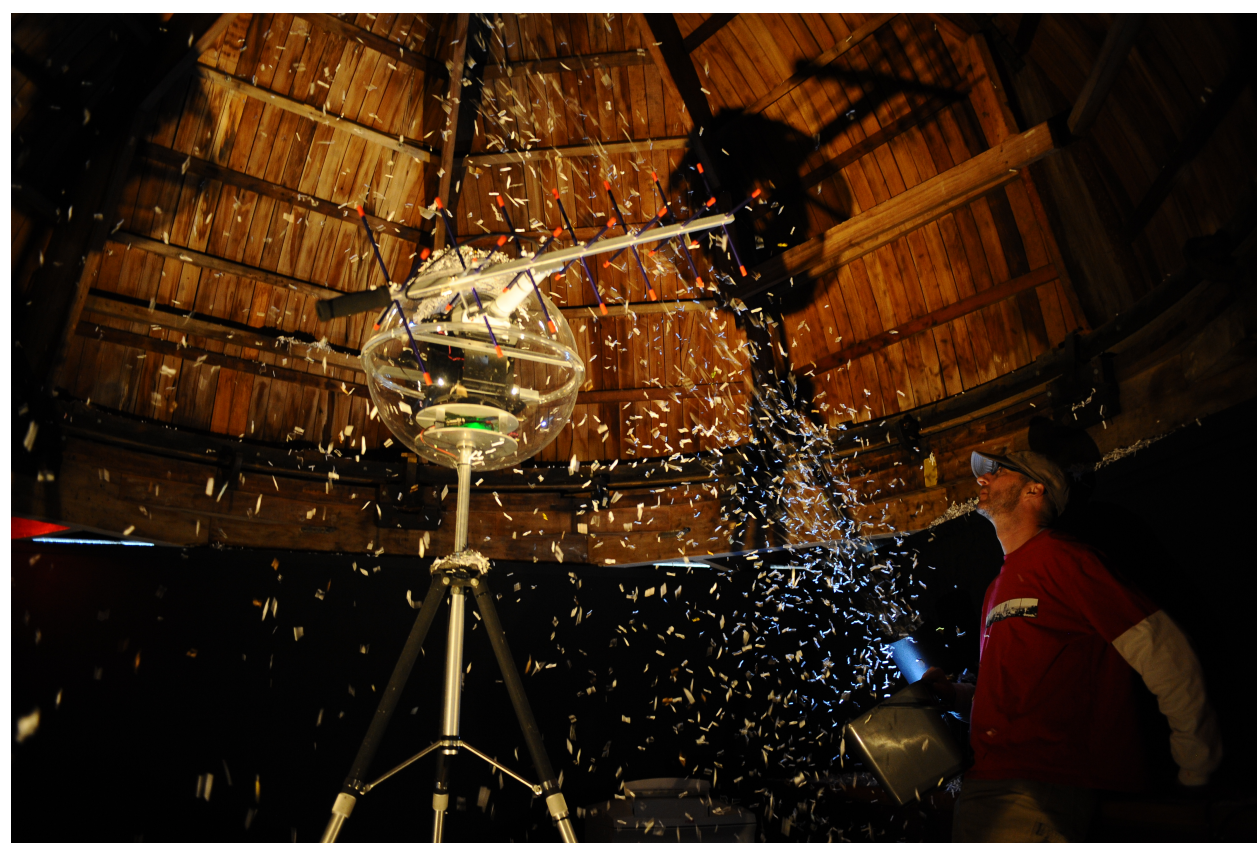

Figure 53: Meet 2 Delete Confetti Cannon

Note. Firing paper shreds in a confetti cannon lit by strobe.

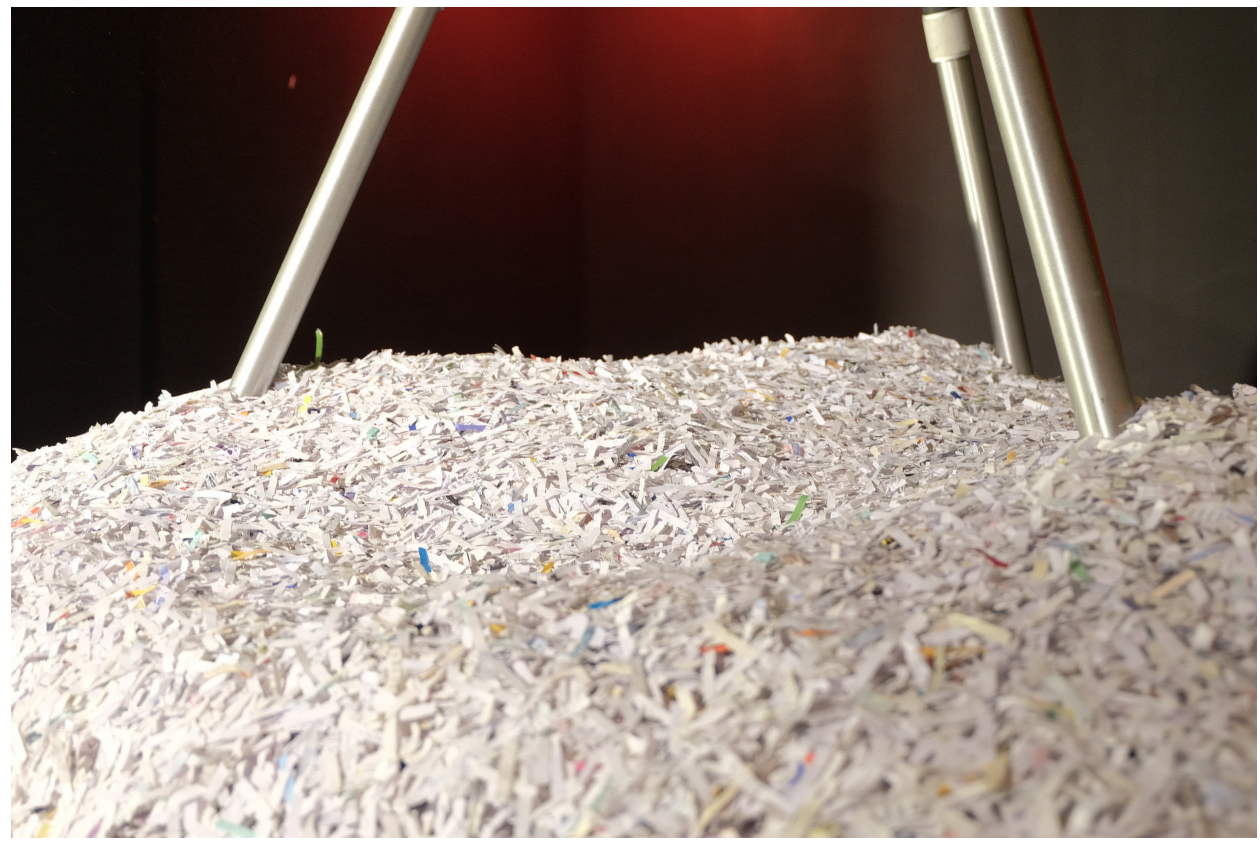

Figure 54: Meet 2 Delete Paper Shred Drift

Note. The dome slowly filled with drifts of paper to a depth of nearly $1 \mathrm{~m}$. By Julian Priest.

e) Antenna Release Performances

March 14, 2019 11:30 NZDT. Thomas King Observatory, Wellington, NZ 


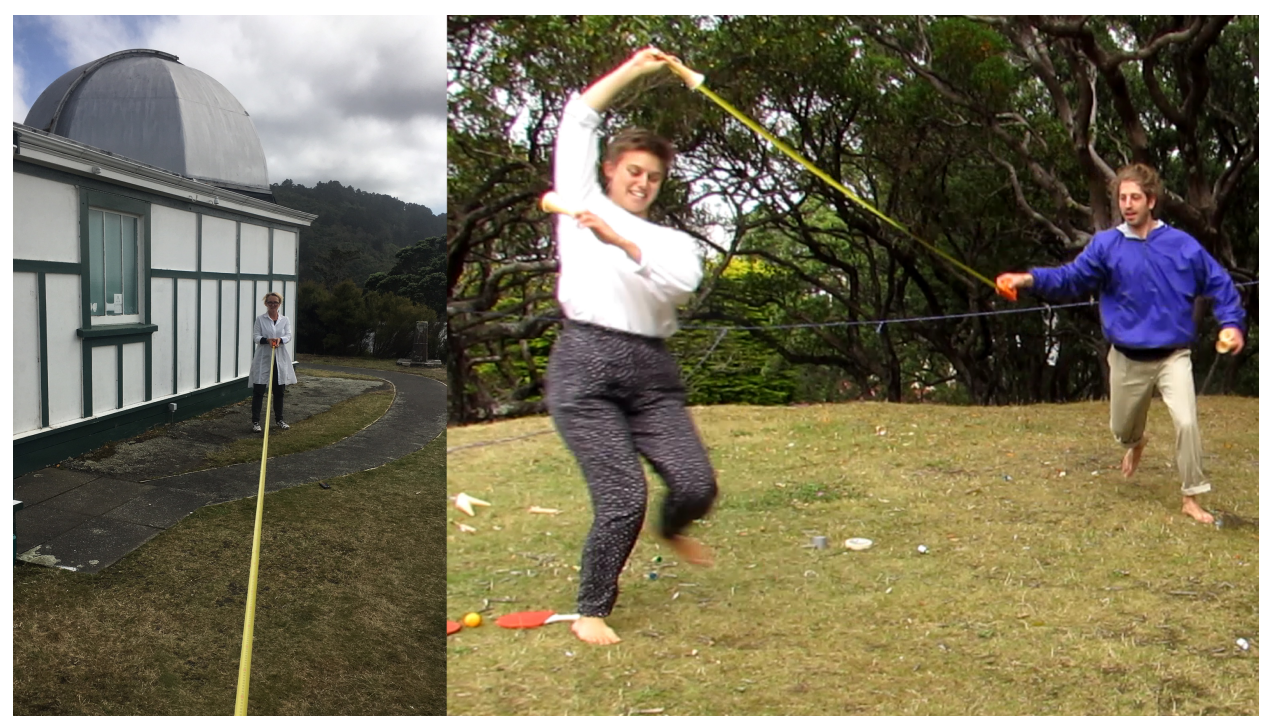

Figure 55: Tape Measure Dance Performances

Note. Two tape measure performances; Sophie Jerram and Bettina Lamm (left) and Adam Ben Dror and Joanne Hobern (right). Collage by Julian Priest. Photos by Bettina Lamm and Julian Priest. With permission.

After Kicksat2 had been deployed there was a malfunction with the satellite's antenna which was made from a tape measure and did not initially extend. The Kicksat was unable to release the Sprite satellites including TWOi without a signal from the ground and so it seemed that the mission would be unsuccessful once more. The Kicksat team attempted to reboot the satellite and send the deploy command using a variety of powerful radio transmitters. Two groups of visiting artists performed impromptu dance rituals at the TKO to 'release' the antenna. Adam Ben Dror and Joanne Hobern of the Playhouse did a dance performance during a satellite transit to an improvised synthesizer sound track, whilst Sophie Jerram and Bettina Lamm performed a dance with a tape measure. Coincidentally the Kicksat Sprites were successfully deployed on March 19th 2019 and a confirmation message was received by the Kicksat team.

f) TWOi 2.0 Autonomous Orbit

March 22, 2019, 19:55:09 UTC. Dwingeloo Radio Telescope, Netherlands 


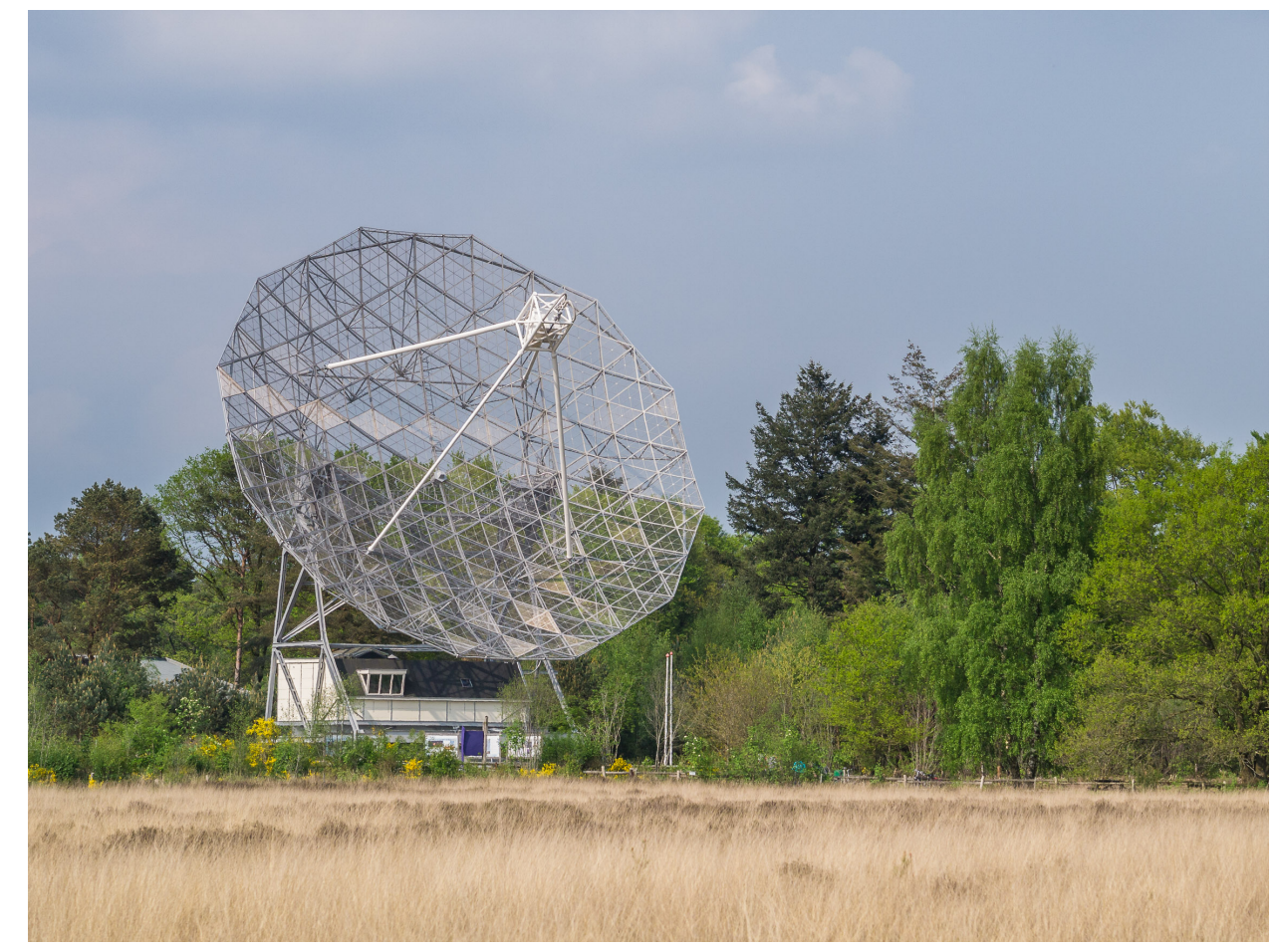

Figure 56: Dwingeloo Radio Telescope

Note. Dwingeloo Radio Telescope. By Uberpruster (2014). Creative Commons Attribution-ShareAlike 3.0 Netherlands).

Only one radio transmission has to date been publicly confirmed from the sprites themselves. The transmission recording was made using a plane sweep of the 23m Dwingeloo Radio Telescope and appeared separated from Kicksat by 5 minutes (Cees Bassa, 2019). Subsequent analysis of the transmission by Esteves (2019), confirmed the presence in the recording of PRN 26 which was the radio signature of TWOi 2.0. This confirmed that TWOi was activated and transmitting from its own autonomous orbit. A single bit of data, representing a zero was the only recorded transmission from the satellite. See Appendix-B for more detail. 


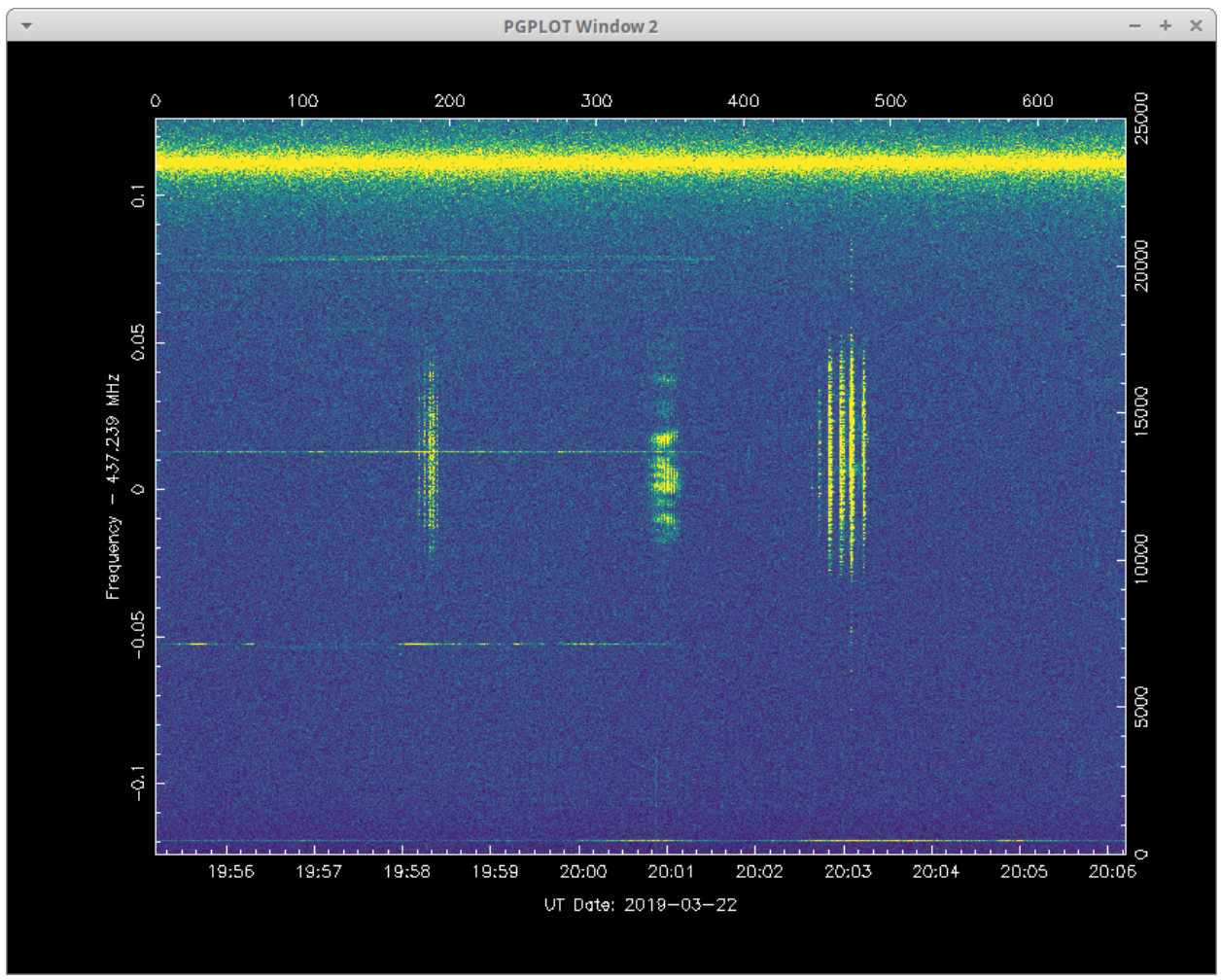

Figure 57: Kicksat Sprite Transmission including TWOi 2.0

Note. Plot of radio transmission received by Dwingeloo Radio Telescope.By (Bassa \& Dijkema, 2019). With Permission. 


\section{the weight of information TWOi flies free 2019-03-23}

Figure 58: TWOi Flies Free Poster

Note. Letter press printed poster celebrating TWOi 2.0 autonomous orbit produced on receipt of the confirmation transmission from Dwingeloo. The poster was produced on March 232019 NZDT which was March 222019 UTC. By Julian Priest.

g) Re-entry

April 2, 2019 05:46 UTC. Upper Atmosphere

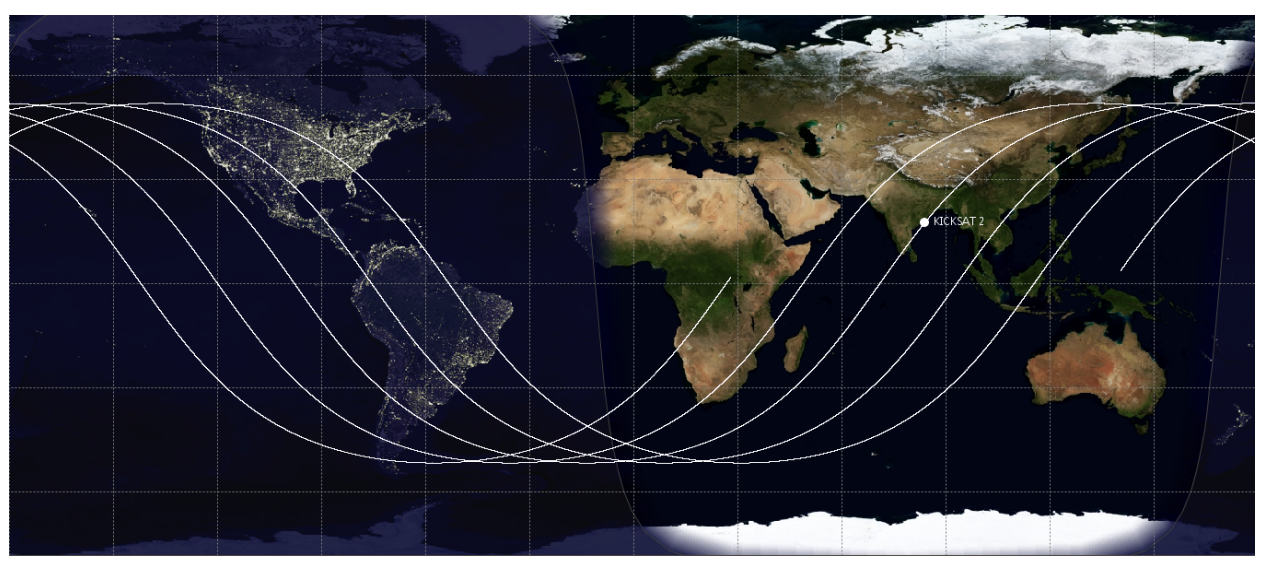


Figure 59: TWOi 2.0 Re-entry Prediction

Note. Kicksat2 de-orbit prediction. By Jon Mikel (2019). With permission.

The exact time and location of TWOi 2.0's de-orbit is unknown, but is estimated to have been on April 22019 Mikel (2014).

h) 13th Conference of the Society for Science Literature and the Arts.

June 27, 2019. Open Hellenic University, Athens, Greece

The TWOi artwork and experimental results were presented at SLSA EU 13. Commentators suggested that further rigorous exploration of the speculative physics experiment aspect of the work could be warranted. 


\section{Appendix B}

\subsection{Speculative Physics Experiment}

A speculative physics experiment was flown on the TWOi satellite and is included here to support the preceding description of the TWOi artwork. There were some preliminary results from the experiment but a detailed discussion is beyond of the scope of this document.

In the TWOi artwork the satellite was characterised as a tragic hero living in a universe in which gravitation is caused by information and not mass. The satellite is described as attempting to ascend to a higher orbit by deleting information from its memory, as if the act of deletion would cause a change in the orbit.

In order to support this story an experiment was designed and flown on both 2014 and 2018 flights. The experiment was designed to test a hypothesis that information deletion could effect orbital dynamics.

The classical view is that composition of a material and hence information should not effect forces due to gravity (Roll, Krotkov, \& Dicke, 1964). There are however theories that explore connections between gravitation and information (Verlinde, 2011; Vopson, 2019), as well as some empirical studies (Kish, 2008).

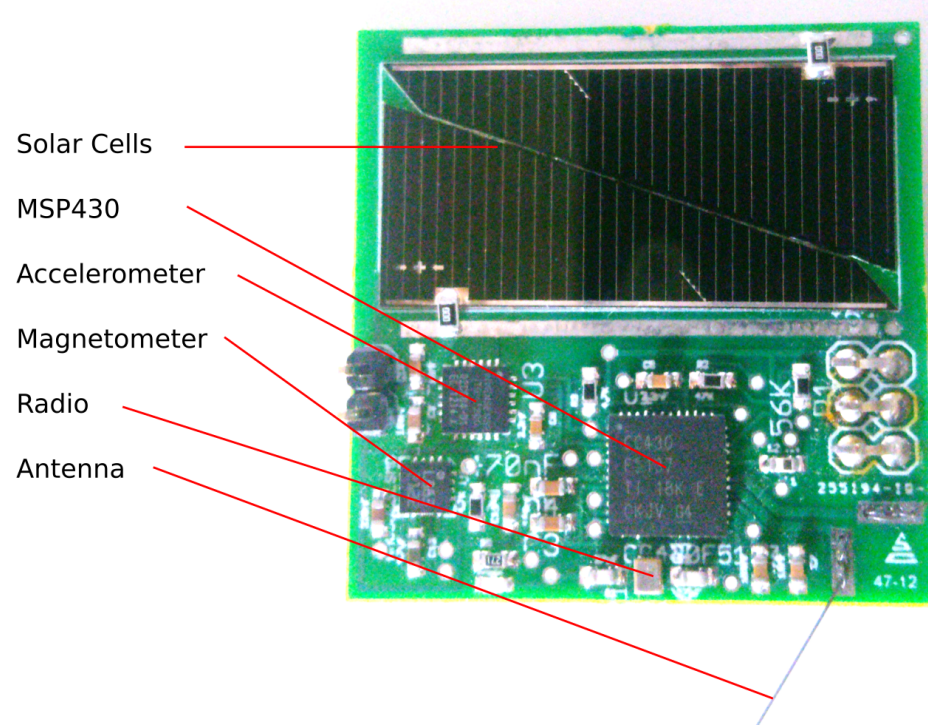

Figure 60: Kicksat Sprite Satellite

Note. Development version of a Kicksat Sprite satellite showing components. By Julian Priest. 
The TWOi satellite ran a version of the Kish (2008) experiment. It was programmed to cycle through a loop in which it would collect data from its sensors, fill its memory with data, measure the character entropy of the data, erase the data and then transmit some simple metrics about its progress from Low Earth Orbit to a ground station.

The TWOi satellite was one of $\sim 100$ identical satellites launched simultaneously and TWOi was the only one running this deletion routine. If the transmission and re-entry timing of TWOi was different relative to the other satellites, this could point to the deletion routine having an effect.

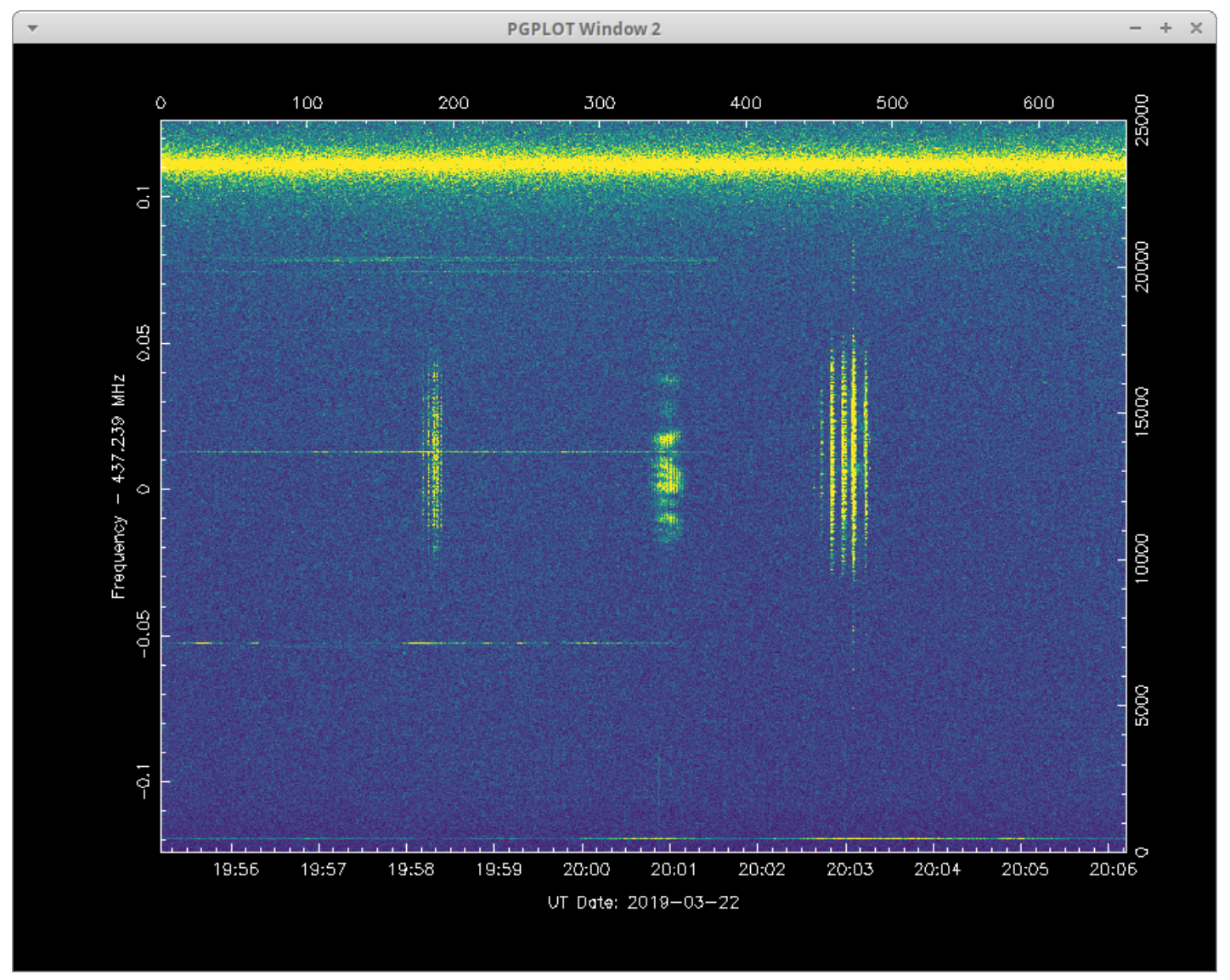

Figure 61: Kicksat Sprite Transmission including TWOi 2.0

Note. Plot of radio transmission received by Dwingeloo Radio Telescope.By (Bassa \& Dijkema, 2019). With Permission.

Transmissions were decoded from only 5 of the satellites and TWOi was observed last or second last of the 5 observed with the other satellites assumed to have de-orbited prior. The transmission represented a single bit - a zero (Bassa \& Dijkema, 2019; Esteves, 2019). 


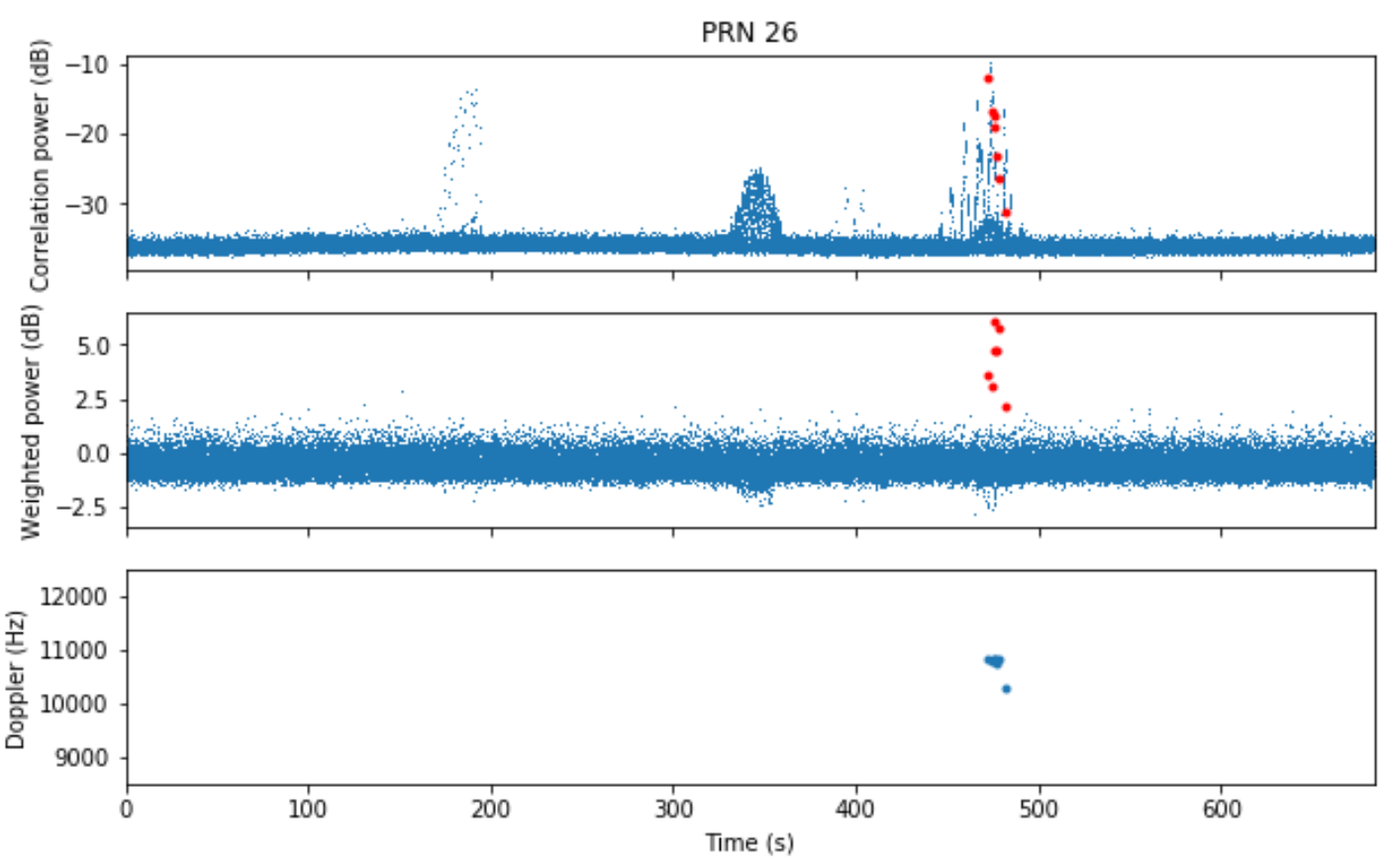

Figure 62: TWOi PRN 26 detection trace

Note. TWOi transmissions were identified by PRN codes 26/27. Analysis by Esteves (2019) shows TWOi was active and transmitting. By Esteves (2019). With permission.

It should again be stressed that this was a speculative experiment intended to express an artistic idea only, rather than a rigorously designed physics experiment. The lack of data makes the experiment inconclusive either way, and further work is planned on this topic in more controlled circumstances. 